\title{
Crustal cracks in areas of active deformation: Correlation of GPS and seismic anisotropy
}

\author{
Katharina Unglert \\ May 16, 2011
}

A thesis submitted to Victoria University of Wellington in fulfilment of the requirements for the degree of Master of Science in Geophysics 


\begin{abstract}
Seismic anisotropy in the upper crust can be observed from shear-wave splitting. It is closely related to crack distribution, orientation and density via the orientation of fast polarisation and the delay time between the two perpendicular components of the original shear-wave. Since stress variations can affect crustal cracks, they should change shear-wave splitting. Another observable result of variations in stress can be deformation measured by GPS (Global Positioning System). Although there are as yet few publications linking these different methods, some have suggested an alignment of fast direction with maximum horizontal compressive stress and maximum horizontal compressive strain.

We examine whether we can observe this or a different relation of seismic anisotropy to strain and stress changes in three different settings. We performed shear-wave splitting analyses of local earthquakes and baseline and strain calculations around Taupo caldera (New Zealand), Aso caldera (Japan), and around an area on the Raukumara peninsula (New Zealand) associated with slow slip on the Hikurangi subduction interface. Both anisotropy and deformation vary with time in all three regions, but the time variations do not strongly correlate with each other. We suggest that a strong deformation signal observed at Taupo caldera might have a regional, non-volcanic source, and small variations in shear-wave splitting cannot be linked to variations in GPS time series or baselines. At Aso caldera strong deformation seems to be directly related to volcanic activity. Seismic anisotropy also shows a change, but at a slightly different time than the GPS signal. On the Raukumara peninsula, the strong deformation associated with slow slip does not show up as a variation in seismic anisotropy, although variations in shear-wave splitting do exist in this area.

Overall, we observe an alignment of fast direction with either maximum horizontal compressive strain or stress or both for a subset of time periods and stations. In addition, there is a contribution of structure to the observed anisotropy. We conclude that deformation and seismic anisotropy cannot always be linked in a straightforward way. Instead, shear-wave splitting may be connected to smaller scale processes than can be detected by the current densities of the GPS networks.
\end{abstract}




\section{Acknowledgements}

The planning of this project, the work associated with it and finally this thesis have been a big part of my life for the last two years. None of it would have been possible without the contribution of all the people and institutions mentioned below.

First of all, thanks to my supervisors Martha and Nico. Both of you were always open for my questions, concerns, ideas, suggestions and just general discussion. You managed to keep me excited about my research and were just the perfect guides through what I consider my first proper research project.

To go back to the very beginning, thanks to faculty and students at the University of Munich, particularly Hans-Peter Bunge, Oliver Spieler, Stuart Gilder and Rocco Marlservisi for encouraging me to go to New Zealand and for supporting me with various applications. Thanks also to Bettina, Jo, Katja, Lena, Leo, Melli, Peter, Sandra, Sarah, Stephan and many more for never losing patience and keeping in touch even over more than $18,000 \mathrm{~km}$ distance.

Thanks to all my friends here in Wellington, especially Aiko, Andrew, Ben, Caro, Giorgi, Johanna, Karo, Karsten, Markus, Martin, Micha, Michelle, Michi, Miri, Philipp and my flatmates at the Wai-te-ata Apartments and on the Terrace for an awesome time in New Zealand! Thanks also to my whole family: Mum, Dad, Jo and Susi, Grosspapa and Omi, my godfather Holm, Babsi, Peter and everyone else for letting me go, visiting me and for supporting me over the entire time. Thanks to Danny for meeting this incredible across-the-ocean-challenge with me!

Thanks to Anya, Dan, Denise, Karen, Laura, Lloyd, Nicholas, Nick, Richard, Roopa, Sanne, Sofia, Syuhadda for being great office mates and good times. Thanks also to Adam, Jess, Katrina, Mark and Sapi, especially for fun fieldwork! Thanks to everyone on the NZ Ocean Bottom Seismic Project cruise on the RV Roger Revelle, to the New Zealand Geophysical Society and to everyone at the Summer of Applied Geophysical Experience in Santa Fe, New Mexico, for giving me simply amazing experiences.

Thanks to Adrian Benson, Nestor Cardozo, Mark Chadwick, Andrew Mellanby, Brad Scott, Steve Sherburn and Euan Smith for help with computers, codes, data and discussion. Thanks also to Gill and Art Jolly for making my stay in Taupo a lot easier.

Last but not least, in relation to the entire project I gratefully acknowledge the generous financial support from the German Academic Exchange Service (DAAD), the German National Academic Foundation, Victoria University of Wellington, the Marsden Fund, the Japan Society for the Promotion of Science and the data provided by the GeoNet project, the Geospatial Information Agency of Japan (GSI), and Aso Volcanological Laboratory. 


\section{Contents}

$\begin{array}{ll}\text { 1. Introduction: Background and Motivation } & 1\end{array}$

1.1. Volcanoes in the past and today . . . . . . . . . . . . . . . . . . . 1

1.2. Monitoring volcanoes . . . . . . . . . . . . . . . . . . 2

1.3. Goals of this study . . . . . . . . . . . . . . . 3

2. Deformation 5

2.1. Basic principles of GPS . . . . . . . . . . . . . . 5

2.1.1. Signal and measurement . . . . . . . . . . . . . . 6

2.1.2. Errors . . . . . . . . . . . . . . . . 7

2.2. Deformation and stress . . . . . . . . . . . . . . . . . . . 10

2.3. Sources of deformation . . . . . . . . . . . . . . . . 11

2.3.1. Tectonic deformation . . . . . . . . . . . . . . 12

2.3.2. Volcanic deformation . . . . . . . . . . . . . . 12

3. Seismic Anisotropy 14

3.1. What is shear-wave splitting? . . . . . . . . . . . . . . . . 14

3.2. Causes of shear-wave splitting . . . . . . . . . . . . . . . 17

3.3. Shear-wave splitting in relation to stress and strain . . . . . . . . 18

3.3.1. Stress . . . . . . . . . . . . . . . . 18

3.3.2. Strain . . . . . . . . . . . . . . . . 19

4. Geology and tectonic settings $\quad 21$

4.1. Taupo Volcanic Zone . . . . . . . . . . . . . . . . . . . . . . . . . . . 21

4.1.1. The Central Volcanic Region . . . . . . . . . . . . . . . . . 21

4.1.2. Taupo caldera . . . . . . . . . . . . . . . . . 23

4.2. Kyushu, Japan . . . . . . . . . . . . . . . . . . . . . . 24

4.2.1. Aso Volcano . . . . . . . . . . . . . . . . . . . . . 25

4.3. The Raukumara peninsula . . . . . . . . . . . . . . . . 26

5. Shear-wave splitting and GPS processing techniques 28

5.1. Automatic splitting technique . . . . . . . . . . . . . . . 28

5.1.1. Silver and Chan method . . . . . . . . . . . . . 28

5.1.2. Problems and limitations . . . . . . . . . . . . . . . . . . 29

5.1.3. Teanby cluster analysis . . . . . . . . . . . . . . . . 30

5.1.4. Automated shear-wave splitting . . . . . . . . . . . . . . . 31

5.1.5. $v_{P} / v_{S}$-ratio calculation . . . . . . . . . . . . 33

5.2. Positioning processing for different regions . . . . . . . . . . . . . . . . . . . . . . . 33

5.2.1. Time series . . . . . . . . . . . . . . . 35

5.2.2. Baselines . . . . . . . . . . . . . . 36

5.2.3. 2D strain . . . . . . . . . . . . . . 36 
6. Data acquisition and processing parameters 38

6.1. Taupo caldera . . . . . . . . . . . . . . . . . . 38

6.1.1. Seismic data . . . . . . . . . . . . . . . 38

6.1.2. GPS data . . . . . . . . . . . . . . . 42

6.2. Aso caldera . . . . . . . . . . . . . . . . . 45

6.2.1. Seismic data . . . . . . . . . . . . . . . 45

6.2.2. GPS data . . . . . . . . . . . . . . . . . . . . . . . . . . . . . . . .

6.3. Gisborne . . . . . . . . . . . . . . . . 51

6.3.1. Seismic data . . . . . . . . . . . . . . 51

6.3.2. GPS data . . . . . . . . . . . . . . . . 5 53

7. Results $\mathbf{5 5}$

7.1. Taupo caldera . . . . . . . . . . . . . . . . 55

7.1.1. Shear-wave splitting . . . . . . . . . . . . 55

7.1.2. Deformation: time series and strain . . . . . . . . . . . . 61

7.2. Aso caldera . . . . . . . . . . . . . . . . . . 67

7.2.1. Shear-wave splitting . . . . . . . . . . . . . . 67

7.2.2. Deformation: time series and strain . . . . . . . . . . 70

7.3. Gisborne . . . . . . . . . . . . . . . . . . 73

7.3.1. Shear-wave splitting . . . . . . . . . . . . . 73

7.3.2. Deformation: time series and strain . . . . . . . . . . 76

$\begin{array}{lr}\text { 8. Discussion } & \mathbf{8 3}\end{array}$

8.1. Taupo caldera . . . . . . . . . . . . . . . . . 83

8.1.1. Influences on shear-wave splitting results . . . . . . . . . . 83

8.1.2. Deformation . . . . . . . . . . . . . . . . 91 91

8.1.3. Integrating GPS and shear-wave splitting . . . . . . . . . . 93

8.2. Aso caldera . . . . . . . . . . . . . . . . . . . . . 94

8.2.1. Influences on shear-wave splitting results . . . . . . . . . . . 94

8.2.2. Deformation . . . . . . . . . . . . . . . . 101

8.2.3. Integrating GPS and shear-wave splitting . . . . . . . . . 102

8.3. Gisborne . . . . . . . . . . . . . . . . . . . . . . 113

8.3.1. Influences on shear-wave splitting results . . . . . . . . . . . 113

8.3.2. Deformation . . . . . . . . . . . . . . . 113

8.3.3. Integrating GPS and shear-wave splitting . . . . . . . . 115

9. Conclusions

9.1. Lessons from GPS and splitting . . . . . . . . . . . . . . . 118

9.1.1. Do shear-wave splitting parameters change over time? . . . . . 118

9.1.2. Do positioning data show temporal changes? . . . . . . . . . . 118

9.1.3. Are these changes (if existent) correlated? . . . . . . . . . . . 119

9.1.4. What can we infer about structure, local and regional stresses? 119 
9.1.5. Are there remarkable differences or similarities in the two data types for different volcanic and non-volcanic environments? . . 120

9.2. Eruption sequence at Aso volcano . . . . . . . . . . . . . . . 120

9.3. Further work . . . . . . . . . . . . . . . . . . . . . . . . . 121

9.3.1. Taupo caldera . . . . . . . . . . . . . . . 121

9.3.2. Aso volcano . . . . . . . . . . . . . . . . . . . . 122

9.3.3. Raukumara peninsula . . . . . . . . . . . . . . . . 123

9.3.4. Amendments to the method . . . . . . . . . . . . . . . 124

$\begin{array}{lr}\text { A. Errors in strain fields } & 126\end{array}$

$\begin{array}{ll}\text { B. Cross correlation of seismograms } & 129\end{array}$

$\begin{array}{ll}\text { C. Correlation of splitting parameters } & \mathbf{1 3 0}\end{array}$

$\begin{array}{ll}\text { D. Moving means at Aso caldera } & 134\end{array}$

$\begin{array}{ll}\text { E. Calculations for stress gradient } & 138\end{array}$

\section{List of Figures}

1.1. Artistic impression of the Hawaiian goddess Pele and her fires. . . . . 1

1.2. Schematic illustration of the connection between deformation and seismic anisotropy . . . . . . . . . . . . . . 3

2.1. Different end-member deformation processes . . . . . . . . . . . 5

2.2. Multipathing of GPS signal . . . . . . . . . . . . . . . . . 9

2.3. Illustration of stress and strain directions in comparison to different symmetry axis orientations . . . . . . . . . . . . . . 11

3.1. Principle of shear-wave splitting for near-vertical plane cracks . . . . 15

3.2. Schematic illustration of open and closed cracks due to stress . . . . . 19

4.1. Setting of Taupo caldera within the TVZ . . . . . . . . . . . . . . 22

4.2. Tectonic setting of Kyushu island, Japan . . . . . . . . . . . . 25

4.3. Tectonic setting of the Raukumara Peninsula . . . . . . . . . . . . . . 27

5.1. Sketch illustrating the principle of cycle skipping . . . . . . . . . . . 30

5.2. Illustration of the difference between within-cluster variance $\sigma_{c}{ }^{2}$ and

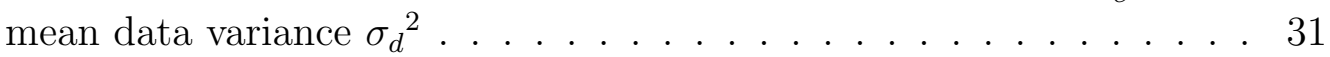

5.3. Summary plot for splitting and event parameters . . . . . . . . . 33

6.1. Station network around lake Taupo, New Zealand . . . . . . . . . . . 38

6.2. Examples for data quality at different stations . . . . . . . . . . . . 41

6.3. Days with shear-wave splitting measurements at the different stations around lake Taupo. . . . . . . . . . . . . . . . . . . . . . 43

6.4. GPS time series for station TAUP . . . . . . . . . . . . . . . 44

6.5. Station network around Aso caldera, Japan . . . . . . . . . . . . . 47 
6.6. Moving means over $\phi$ and $\delta t$ for station TAT . . . . . . . . . . . . . 47

6.7. GPS timeseries for station 021072 at Aso caldera . . . . . . . . . . . 49

6.8. Station network in the area of the Gisborne slow slip events . . . . . 51

6.9. GPS timeseries for station GISB showing slow slip events . . . . . . . 53

7.1. Used earthquakes vs. all downloaded events for lake Taupo . . . . . . 55

7.2. 30 point moving means for $\phi$ and $\delta t$ at stations HATZ and KATZ . . 58

7.3. 30 point moving means for $\phi$ and $\delta t$ at stations RATZ and WATZ . . 59

7.4. Illustration of the difference between mean and mode for non-gaussian distribution . . . . . . . . . . . . . . 6 60

7.5. Mode for moving 15 day windows at station RITZ . . . . . . . . . . . 61

7.6. Rose diagrams for crust and mantle events for Taupo caldera 2006-2008 62

7.7. Filtered GPS timeseries for Taupo caldera . . . . . . . . . . . . . 63

7.8. Two examples for baseline changes around lake Taupo . . . . . . . . . 65

7.9. Strain field for Taupo caldera . . . . . . . . . . . . . . 66

7.10. Earthquakes that yielded A and B grade splitting measurements at Aso caldera. . . . . . . . . . . . . . . . . . . 6 67

7.11. Moving means over splitting parameters and $v_{P} / v_{S}$-ratio at station TAT .......................... 70

7.12. Shear-wave splitting fast directions for shallow and deep subset at Aso caldera . . . . . . . . . . . . . . . . . 71

7.13. Baseline changes for station pairs 960703-960701 and 960702-950474 at Aso caldera . . . . . . . . . . . . . . . . . . . 72

7.14. Strain field for all periods at Aso caldera . . . . . . . . . . . . . . 74

7.15. Earthquakes with A or B grade splitting measurements for the Raukumara Peninsula . . . . . . . . . . . . . . 75

7.16. Moving means over $\phi$ and $\delta t$ at station KNZ, Raukumara Peninsula . 77

7.17. Moving means over $\phi$ and $\delta t$ at station PUZ, Raukumara Peninsula . 78

7.18. Rose diagrams for Raukumara Peninsula . . . . . . . . . . . . . . . 80

7.19. GPS timeseries raw and filtered for station GISB . . . . . . . . . . 81

7.20. Strain field for Raukumara peninsula, 2009 - 2010 . . . . . . . . . . . 82

8.1. Different parameters at station HATZ vs. dominant seismogram frequency . . . . . . . . . . . . . . . . . 8 85

8.2. Comparison of moving means over results from only the best filter and all three filters . . . . . . . . . . . . . . . 886

8.3. Dependencies of $\phi$ and $\delta t$ on earthquake parameters, Taupo caldera. . 88

8.4. GPS timeseries for stations TAUP and RGHR . . . . . . . . . . . . . 92

8.5. Comparison of GPS timeseries and shear-wave splitting parameters at lake Taupo . . . . . . . . . . . . . . . . . . . . . 93

8.6. Location of earthquake clusters at Aso caldera . . . . . . . . . . . . 95

8.7. Moving means over results from clusters at station TAT . . . . . . . . 97

8.8. Dependencies of $\phi$ and $\delta t$ on earthquake parameters at Aso caldera . 98

8.9. $P$ - and $S$-picks at station HDK for event 0107040506 . . . . . . . 100 
8.10. Depth distribution of earthquakes with splitting measurements for Aso caldera . . . . . . . . . . . . . . . . . . 106

8.11. Best fitting stress models at Aso caldera, periods I-IV . . . . . . . . . 107

8.12. Best fitting stress models at Aso caldera, periods V-VI . . . . . . . . 108

8.13. Monitoring parameters from Nakadake first crater . . . . . . . . . . . 110

8.14. Dependencies of $\phi$ and $\delta t$ on earthquake parameters for Raukumara peninsula . . . . . . . . . . . . . . . . . . 114

8.15. Shear-wave splitting fast directions at $5 \mathrm{~km}$ depth . . . . . . . . . . 117

9.1. Schematic illustration of fluid circulation related to eruption sequence at Aso caldera . . . . . . . . . . . . . . . . . . . . . . . 122

A.2. Inversion errors from SSPX for Raukumara peninsula . . . . . . . . . 126

A.3. Inversion errors from SSPX for Aso caldera . . . . . . . . . . . . . 127

A.4. Inversion errors from SSPX for lake Taupo . . . . . . . . . . . . . . . 128

C.5. Correlation coefficient and p-value matrices for station ARAZ . . . . 132

C.6. Correlation coefficient and p-value matrices for station ARAZ . . . . 133

D.7. 30 point moving means at Aso caldera: HDK and MAK. . . . . . . . 135

D.8. 30 point moving means at Aso caldera: MGR and MKN. . . . . . . 136

D.9. 30 point moving means at Aso caldera: NBR and SUN. . . . . . . . . 137

\section{List of Tables}

6.1. Summary of station specifications, Taupo caldera . . . . . . . . . . 39

6.2. Summary of cGPS station specifications, Taupo caldera. . . . . . . 39

6.3. Definition of episodes for Taupo caldera . . . . . . . . . . . . . . 43

6.4. Summary of station specifications at Aso caldera . . . . . . . . . . 46

6.5. Summary of GPS station specifications at Aso caldera . . . . . . . . 48

6.6. Definition of episodes for Aso volcano, based on shear-wave splitting and strain analyses . . . . . . . . . . . . . . . 5 50

6.7. Parameters for SSPX strain calculation for Aso volcano . . . . . . 50

6.8. Summary of stations specifications, Gisborne area. . . . . . . . . . . 52

6.9. Summary of GPS station specifications, Raukumara Peninsula. . . . 52

6.10. Parameters for SSPX strain calculation for Raukumara Peninsula . . 54

7.1. Summary of parameters from shear-wave splitting at Taupo caldera . 56

7.2. Number of splitting measurements, Taupo caldera . . . . . . . . 56

7.3. Summary of fast directions and delay times for shallow and deep events at lake Taupo . . . . . . . . . . . . . . . . 62

7.4. Summary of directions for strain shortening axes at Taupo caldera for periods IV - VII . . . . . . . . . . . . . . . . . . . . . . 68

7.5. Strain shortening directions at stations around Lake Taupo for periods IV - VII . . . . . . . . . . . . . . . . . . . . . . . 68

7.6. Summary of parameters from shear-wave splitting at Aso volcano . . 69 
7.7. Summary of fast directions and delay times for shallow and deep events at Aso caldera . . . . . . . . . . . . . . . . 71

7.8. Mean, standard deviation, minimum and maximum of $v_{P} / v_{S}$-ratio for 2001-2008 at Aso caldera . . . . . . . . . . . . . . . . 72

7.9. Summary of parameters from shear-wave splitting from the Raukumara peninsula from 2002-2005 . . . . . . . . . . . . . 73

7.10. Summary of parameters from shear-wave splitting from the Raukumara peninsula from 2009-2010. . . . . . . . . . . . . . 76

7.11. Summary of fast directions and delay times on the Raukumara Peninsula 2002-2005 and 2009-2010 . . . . . . . . . . . . . . . 79

7.12. Summary of mean fast directions and delay times on the Raukumara Peninsula for 2009, 2010 during and 2010 after slow slip . . . . . . . . 79

8.1. Summary of characteristics of earthquake clusters found around Aso volcano. . . . . . . . . . . . . . . . . . . . . . 95

8.2. Summary of parameters from shear-wave splitting at Aso volcano, for earthquakes in clusters . . . . . . . . . . . . . . . . . . 996

8.3. Parameters for different stress models . . . . . . . . . . . . . . . . 105

8.4. Fit of the regional stress, splitting and strain . . . . . . . . . . 106

8.5. Best fitting stress models for the different periods at Aso caldera . . . 108

B.1. Input parameters for BCSeis . . . . . . . . . . . . . . . . . 129

C.2. Abbreviations for parameters from splitting measurements used for correlation . . . . . . . . . . . . . . . . . 131 


\section{Introduction: Background and Motivation}

\subsection{Volcanoes in the past and today}

The word "volcano" comes from the Italian volcanic island Vulcano ("burning mountain") in the Mediterranean (Volcano, nd). The volcanic activity of the island was attributed to the fires of the Roman god Vulcanus, a blacksmith. Similarly, volcanism on the islands of the Hawaiian chain has been attributed in various stories to the rage of the goddess Pele and her fires (Swanson, 2008). While in historic

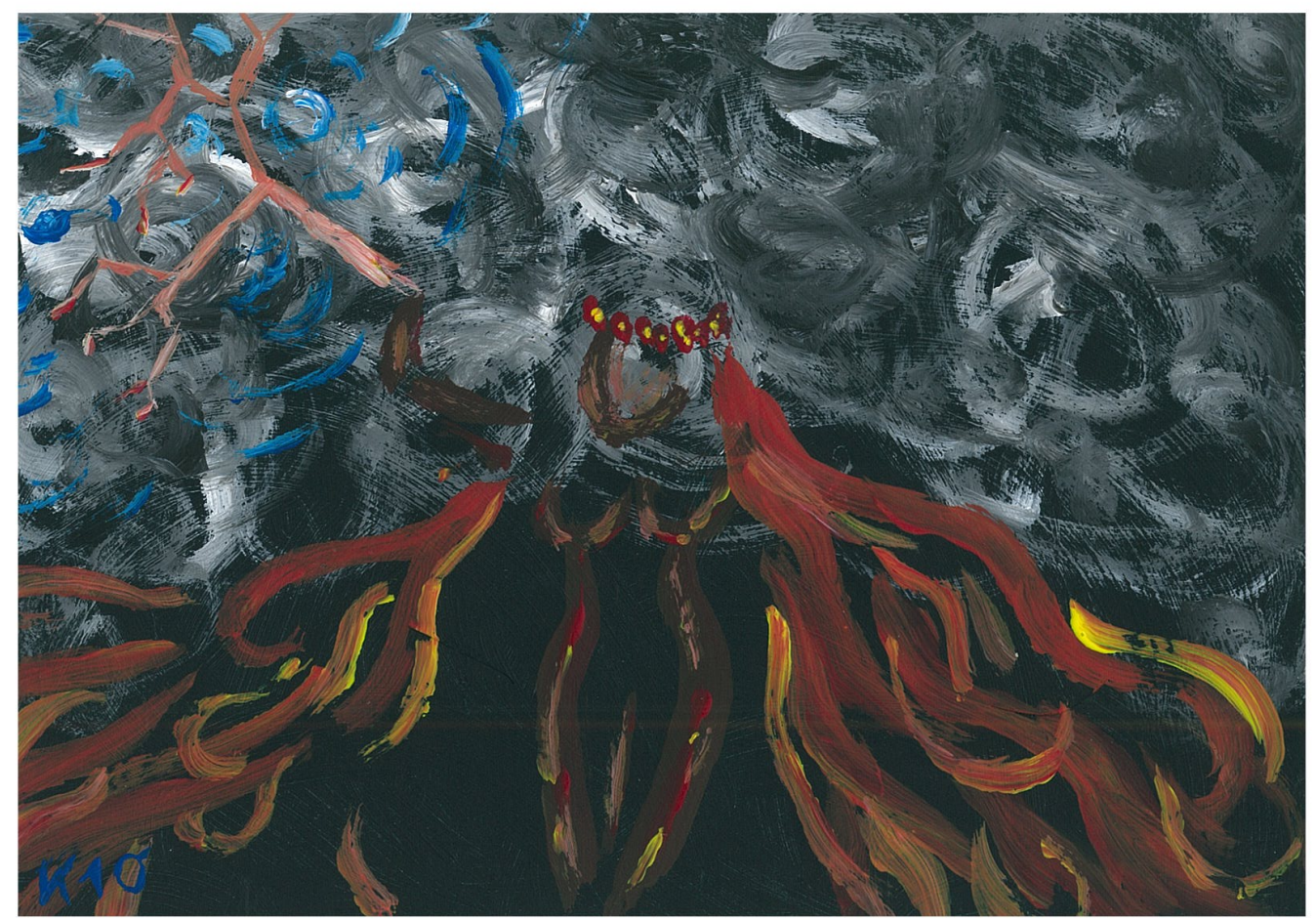

Figure 1.1: Artistic impression of the Hawaiian goddess Pele and her fires.

times these were the only ways people could explain volcanic activity, the origin and processes leading to and accompanying volcanic eruptions are nowadays understood better and better. Although it is possible to assess volcanic hazards in form of probabilities and we can detect eruptions, sometimes even forecast them within a few days or hours, there are examples of other eruption that took place "out of the blue" without significant prior warning signs (e.g. Jolly et al., 2010). Around the Pacific Ring of Fire, millions of lifes are endangered by volcanic activity every year (e.g. Chester et al., 2001; Tanguy et al., 1998). While for example volcanoes of the Aleutian Chain rarely pose a direct hazard due to the sparsely populated areas in that region, indirect danger arises as major airplane pathways cross the air above these volcanoes (Murray et al., nd; Carn et al., 2009). In other regions, like Japan, millions of people inhabit areas in direct vicinity to volcanic centres (Chester et al., 
2001). As scientists, we need to be able to understand how, when and why volcanic eruptions happen in order to be able to give appropriate advice to civil authorities and enable early warning of local communities. This study makes an attempt to improve our knowledge about volcanic environments and tests a new technique to assess temporal changes in such areas.

\subsection{Monitoring volcanoes}

Earthquakes and volcanoes have been observed together for centuries. Father Goree, a Jesuit, writes about the appearance of volcanic islands around Santorini (Greece): "There was then a New Island, which [...] rose up by the means of Subterraneous Fires, which caused several violent Earthquakes [...]." (Goree, 1710, p.357). Even earlier, in 1693 Martin Hartop observes earthquakes and volcanism on Sicily, Italy and concludes that "it seems highly probable, that these Tremblings of the Earth proceed from the same incens'd matter, which [...] has so furiously broken out in Smoak and Fire." (Hartop, 1693, p.827). Nowadays, we have several means by which we can relate earthquakes and volcanism: While rate of seismicity, different waveforms or earthquake locations have been used to quantify the status of a volcano (e.g. Luckett et al., 2007; Hurst et al., 2004), in this study we are using the fact that earthquake waves recorded on or nearby a volcano most likely will have passed through material affected by the volcano and its associated processes, for example movement of magma or changes in hydrothermal systems. These regions are prone to changes caused by volcanic activity: temperature, fluid or gas content, magma and steam pathways as well as stresses can undergo drastic fluctuations as the volcano goes from one state into another (e.g. Hurst et al., 2004; Dieterich et al., 2000; Wyss et al., 1992).

Although for most purposes, such as earthquake location problems, the earth can be assumed to be isotropic, it is anisotropic, depending on region and scale (Savage, 1999). If shear-waves travel through an anisotropic medium, they split into two quasi perpendicular components with differenct velocities. For studies in volcanic environments, the medium of interest is the crust. With additional information and certain assumptions, we can estimate orientation and densities of cracks from shear-wave splitting parameters (Crampin and Lovell, 1991, and references therein). Crack distribution is influenced by local and regional stresses (e.g. Zatsepin and Crampin, 1997), so possible changes in the stress field can induce changes in local crack systems. This can theoretically be observed in shear-wave splitting.

Since the advent of modern instruments, positioning data can be retrieved continuously on dense grids (e.g. El-Rabbany, 2002). Active volcanoes are of major interest, since eruptions are often preceded, accompanied or followed by deformation episodes. Source of ground displacement can be changes within or movement of magma bodies (e.g. Sudo et al., 2006; Masterlark et al., 2010) or variations associated with fluids and steam pathways (e.g. Watson et al., 2000). These processes are also likely to change the local stress field (see figure 1.2). Hence, it is possible that 
shear-wave splitting and deformation measurements show similar changes in time. If that is indeed the case, both techniques together could be used to improve our understanding about volcanic processes involving stress changes, expand volcano monitoring and possibly get one step closer to forecasting volcanic eruptions.

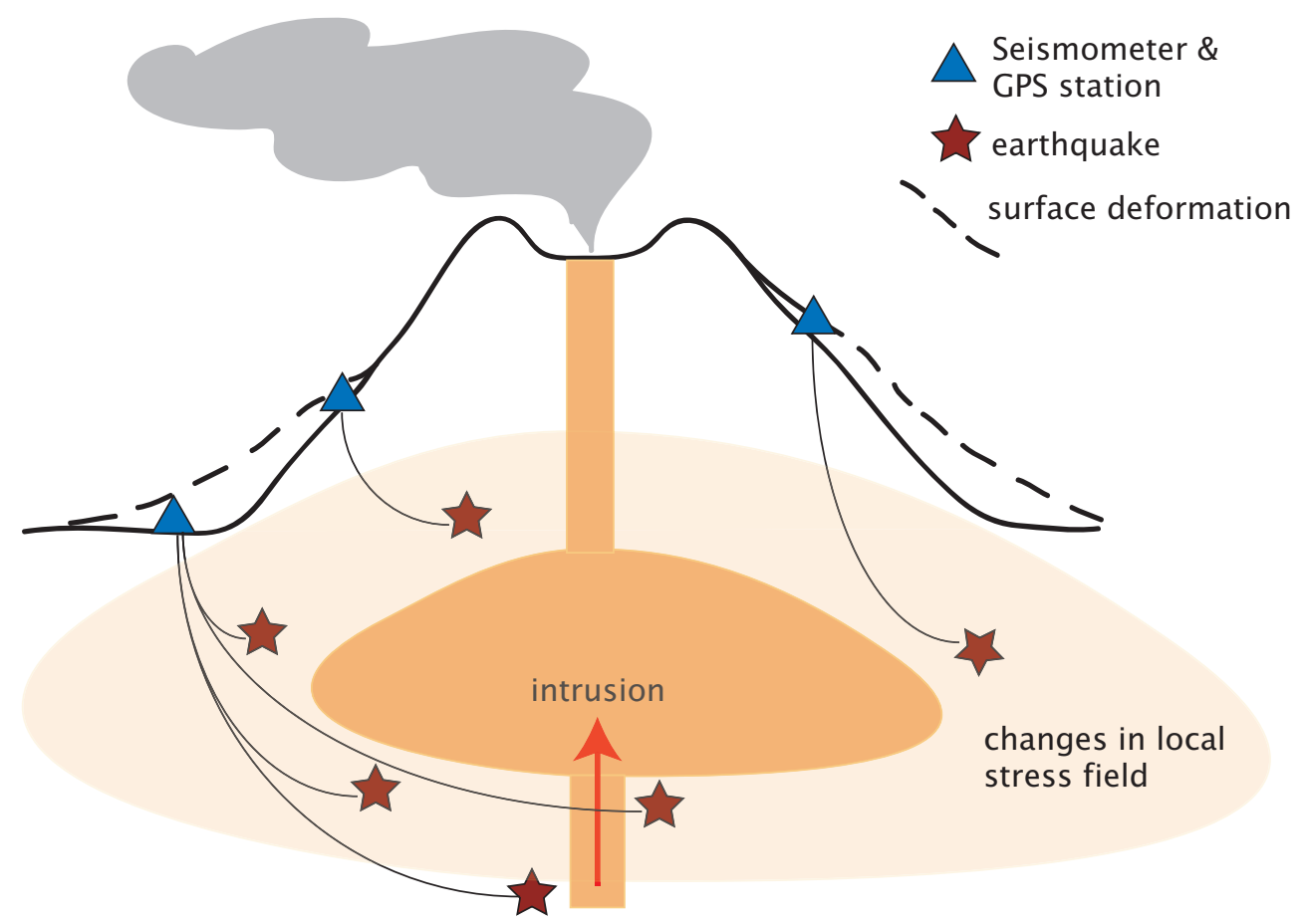

Figure 1.2: Schematic illustration of the connection between deformation and seismic anisotropy in a volcanic setting. A magma intrusion changes the local stress field and crack distribution, and causes surface displacement and a change in seismic anisotropy. Observations are made at GPS stations and seismometers on the surface.

\subsection{Goals of this study}

A previous study on Mt. Asama has succesfully tested shear-wave splitting and GPS (Global Positioning System) data for correlation (Savage et al., 2010b): Changes in baseline over an inferred dyke location correspond to variations in shear-wave splitting delay time with a probability of being related by chance of less than $0.3 \%$.

In this follow-up study we compile data from three different regions and analyse them in terms of shear-wave splitting and positioning data to test if the method can be applied in a straighforward way to other settings than Mt. Asama. The main questions we are going to address are:

- Do shear-wave splitting parameters change over time?

- Do positioning data show temporal changes? 
- Are these changes (if existent) correlated?

- What can we infer about structure, local and regional stresses?

- Are there remarkable differences or similarities in the two data types for different volcanic and non-volcanic environments?

We are going to introduce the volcanic settings of Taupo volcanic caldera in New Zealand, and Aso caldera on Kyushu, Japan. In addition, the Raukumara peninsula, New Zealand, which is an area around episodic slow slip on the subduction interface will be analysed in an attempt to understand a possible relation between deformation and shear-wave splitting. The following sections will describe the basics of GPS and seismic anistropy, give an overview over the different geologic and tectonic settings, review the steps of data collection and processing, interpretation and give some future prospects. 


\section{Deformation}

Surface displacement is observed all over the globe on different time scales. While interseismic plate motion results in movement of one surface point with respect to another on the order of $10 \mathrm{~mm} / \mathrm{yr}$ (DeMets et al., 1994), volcanic and/or hydrothermal systems can show rapid deformation with rates on the order of $1 \mathrm{~m} /$ day (Segall, 2010). As this can be precursory to volcanic eruptions, it is important to monitor deformation, and understand its origins and interactions. This section will explain how we use GPS to measure ground displacement and discuss the processes most likely affecting the areas investigated in this study.

In general the earth's surface can change its shape (i.e. de-form) in three different ways (e.g. Means, 1976): by simple movement of all points with respect to their original position along a vector (translation), by rotating a body (rotation) and by changing the position of the points within a material with respect to each other (distortion) as shown in figure 2.1. All three of them can occur on their own, however, in the earth they will generally appear in combination with each other. We will refer to them with the specific terms stated above if we address only one specific process. The terms deformation and displacement will be used as general terms for any (most likely unknown) combination of the three different types. We will explain how we use GPS to measure the position of points on the earth's surface. By repeatedly obtaining positions potential movement of those points can be detected, which can then be used to quantify ground surface displacement and finally to compute strain. The following sections will go into detail about those measurements and calculations and have largely been compiled from Hofmann-Wellenhof et al. (1993); El-Rabbany (2002); Misra and Enge (2006) and references therein.

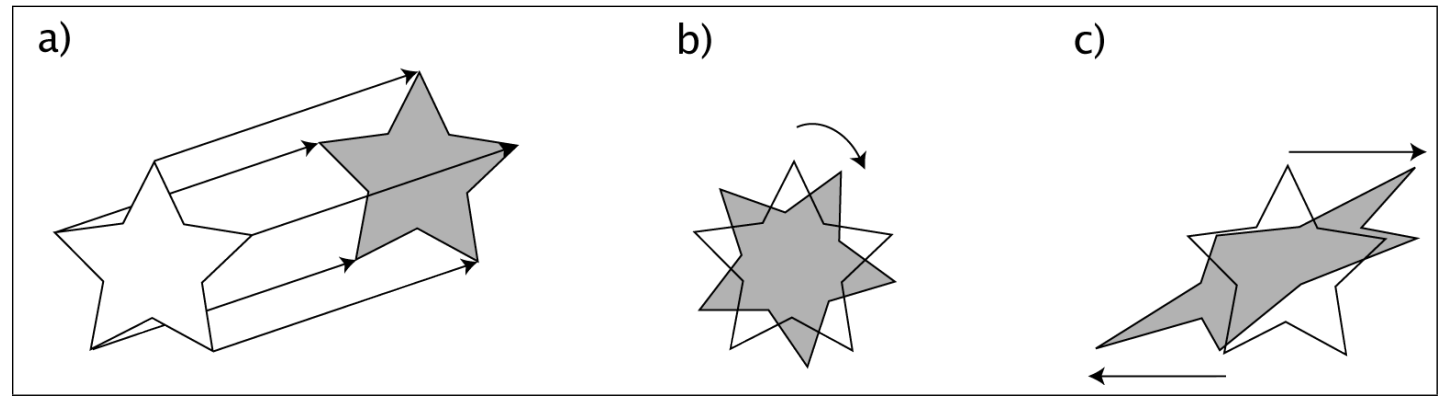

Figure 2.1: Different end-member deformation processes: a) translation, b) rotation and c) distortion. The white star is the undeformed (initial) state, the black arrows indicate how deformation occured and the grey stars show the shape/position after deformation.

\subsection{Basic principles of GPS}

Global Positioning System (GPS) was initially developed as a military tool to determine positions of aircraft and vessels (e.g. Hofmann-Wellenhof et al., 1993). How- 
ever, it has been made available to the public in a slightly limited version free of direct charge (El-Rabbany, 2002). The system consists of three main parts (Misra and Enge, 2006). The control segment monitors atmospheric variations, and predicts satellite tracks and behaviour of the atomic clocks in the satellites, among other tasks. The space segment is made of 24 satellites in six nearly circular orbits inclined at $55^{\circ}$ relative to the equatorial plane approximately $20,000 \mathrm{~km}$ above the earth's surface. The third segment is the user segment; this can be a handheld GPS unit for finding directions, a GPS monument for scientific purposes, or an aircraft, for example. The main idea behind this system is to calculate the distance between a GPS antenna (the user segment) and four to ten satellites with known positions (the space segment) and from these distances determine the position of the GPS antenna.

\subsubsection{Signal and measurement}

The GPS satellites transmit signals on two ultra-high frequency bands: L1 with a centre frequency of $f_{1}=1575.42 \mathrm{MHz}$ and L2 with a centre frequency of $f_{2}=$ 1227.60 MHz (Misra and Enge, 2006). Each signal consists of a sinusoidal carrier phase at either $f_{1}$ or $f_{2}$, a binary ranging code that is unique to each satellite and a binary navigation message. The ranging code can be a coarse/acquisition $(C / A)$ or a precise, encrypted $(P(Y))$ pseudo-random noise signal. While the L1 band carries $\mathrm{C} / \mathrm{A}$ and $\mathrm{P}(\mathrm{Y})$ code, the $\mathrm{L} 2$ band only carries the $\mathrm{P}(\mathrm{Y})$ code and was for US Department of Defense authorised use only. The navigation message contains information about satellite status, position, velocity, clock bias parameters and more. It is combined with the ranging code using an operation called modulo- 2 addition, in which the signal is 0 if the ranging code and the navigation bit are the same and 1 if the two bits have different valus (i.e. 0 and 1 or vice versa). The resulting signal is then modulated on to the carrier phase and can be detected by the receiver (Hofmann-Wellenhof et al., 1993; Misra and Enge, 2006).

In very general terms, the receiver measures the distance between the satellite antenna and the receiver antenna positions (El-Rabbany, 2002). With a rough guess of location and with information from the almanac about satellite tracks, the receiver detects satellites in the area. From the unique ranging code, it can be inferred which signal is coming from which satellite. The receiver then generates the exact same ranging code and measures the time shift of this signal in relation to the one received from the satellite by auto-correlation. Multiplied by the speed of light, this time difference is used to calculate the distance between the two antennas, called pseudorange, and the position can theoretically be determined from the information about satellite positions if three or more satellites are visible. In practice, however, a fourth satellite is needed due to satellite and receiver clock errors, which introduce a fourth unknown in addition to the three unknown coordinates (El-Rabbany, 2002). This type of position determination is called GPS point positioning and has a specified accuracy of $13 \mathrm{~m}$ in the horizontal and $22 \mathrm{~m}$ in the vertical direction within 
95\% confidence limits, respectively (Misra and Enge, 2006). A higher accuracy can be achieved by using differential methods: An additional groundbased receiver with known location can be used to correct for errors introduced due to atmospheric disturbances, for example (see section 2.1.2). Furthermore, velocities can be obtained making use of the Doppler shift of the signal due to the relative movement of the receiver to the satellite. As this study does not use velocities directly measured with GPS, further discussion is omitted here.

For geophysical purposes, we need accuracy on the order of millimeters. This can be achieved by using the carrier signal instead of the ranging code. With a much lower wavelength $(\sim 20 \mathrm{~cm}$ as opposed to $\sim 300 \mathrm{~m}$ and $30 \mathrm{~m}$ for the $\mathrm{C} / \mathrm{A}$ and the $\mathrm{P}(\mathrm{Y})$ code, respectively) the carrier phase can be measured with greater precision (e.g. Misra and Enge, 2006). Despite this advantage, ambiguity is introduced due to the nature of sinusoidal signals: When measuring phase differences, we can only know about the difference in partial cycles, while the integer number of full cycles that two signals might be shifted by remains unknown. This so-called integer ambiguity can be resolved by making use of the motion of the satellite. Misra and Enge (2006) describe the approach in detail; a summary is given here. As the satellite moves, the angle at which the signal arrives at the receiver and hence the difference in fractional cycles change slightly. The integer number of cycles, however, will stay the same if the distance by which the satellite moved is small enough. Having two known, independent fractional cycles and one integer number of cycles, we have a system of two linear equations that can be solved without ambiguity in the 1D case. Expanding this to 3D is simply a matter of more satellites to account for the additional unknowns. A drawback is the associated processing time and hence real-time position estimating is hardly achieved with this method.

\subsubsection{Errors}

As mentioned before, the measurements obtained by GPS are subject to errors that can be grouped into satellite errors, uncertainties arising from variations in the propagation medium and receiver errors.

Satellite errors Satellite tracks are predicted by the control segment on a regular basis. The typical prediction will be based on a moving four hour observation window for each one hour period (El-Rabbany, 2002). Errors are associated with the observation as well as the prediction and can account for wrong satellite position estimates (ephemeris errors) and satellite clock timing errors (Misra and Enge, 2006). Although they are still considerably high for geophysical purposes (on the order of metres according to Misra and Enge, 2006), the data used in this study is postprocessed with known orbits and ephemeral errors should hence be minimal. Timing errors should be the same for relatively small, regional networks such as the ones used here, as all stations within the network should "see" the same satellites and 
hence have the same timing errors. Thus only absolute positions will be affected, which are not as relevant for this study as the relative positions.

Propagational errors $\quad 95 \%$ of the GPS signal travel path is practically in free space. At approximately 1,000 km above the earth's surface, however, the signal enters the dispersive ionosphere, a gaseous region containing layers with different amounts of ions (El-Rabbany, 2002; Misra and Enge, 2006, and references therein). While the code phase is delayed due to the dispersive character of the ionosphere, the carrier phase is advanced by the same amount and the resulting path length estimate is too short; an effect called ionospheric delay (see Hofmann-Wellenhof et al., 1993, Chapter 6.3.2. and references therein for detailed derivation of the refractive indices and corresponding velocity changes). Using both frequency bands L1 and L2 could quantify the effect, as dispersion is frequency dependent. Since this introduces a higher observation noise and creates problems with resolving the integer ambiguity, the more common approach is to correct for the ionospheric delay by using empirical ionosphere models that can remove up to $60 \%$ of the effect (El-Rabbany, 2002). The dispersivity depends on the total electron count in the ionosphere and is controlled by factors such as time of day, seasons and sunspot cycle and can cause pseudorange errors of up to tens of metres (Hofmann-Wellenhof et al., 1993; Misra and Enge, 2006).

At approximately $50 \mathrm{~km}$ above the surface of the earth, the signal enters the neutral atmosphere. Despite the non-dispersive character of this part of the path, errors are introduced in form of velocity variations due to the presence of $\mathrm{N}_{2}, \mathrm{O}_{2}$, minor amounts of other dry gases and water vapor (Misra and Enge, 2006). Most of these gases are assembled in the troposphere at heights of up to $16 \mathrm{~km}$ at the equator and $9 \mathrm{~km}$ at the poles; the error associated with the velocity variation is hence referred to as tropospheric delay. It cannot be corrected for in a straightforward manner because all frequencies experience the same variations. Temperature, pressure and humidity models have to be used instead and pose difficulties particularly concerning the amount of water vapor, which depends weakly on local weather patterns (El-Rabbany, 2002; Miyazaki et al., 2003; Li et al., 2008). Errors can be up to $30 \mathrm{~m}$ for long travel paths (El-Rabbany, 2002), but will not have a big impact on the relative positions of stations used here.

Receiver errors Additional errors are created at the receiver. Cables, amplifier and signal quantization, for example, introduce random noise (Misra and Enge, 2006). According to El-Rabbany (2002), the error arising from the difference between the physical centre of the GPS antenna and the actual point where the signal is received (antenna phase centre offset) can be up to a few centimetres. It can be minimised by using the same antenna type for all receivers in a network and orienting them in the same direction, and it will be negligible over long time spans. Moreover, multipathing can obscure the signal and is reported as the major receiver error (Strang and Borre, 1997). Figure 2.2 illustrates the principle. An incoming signal is recorded twice (or 
more times) - the direct wave as well as reflected phases, which can severely distort the overall signal (El-Rabbany, 2002). Minimisation of this error can be introduced by choosing a suitable receiver location with a low number of possible reflectors in the vicinity and by appropriate antenna design so that for example reflections from the ground will be blocked (Misra and Enge, 2006). Polarisation filters can help, as the line-of-sight GPS signal is right-handed, whereas a reflected phase will have a left-handed polarisation (El-Rabbany, 2002). However, a signal reflected twice will have the original polarisation again and cannot be suppressed with such a filter. Despite these difficulties, Strang and Borre (1997) estimate the maximum error for carrier phase measurements to be less than $5 \mathrm{~cm}$. Furthermore, if positions are estimated every day at the same time the multipath error will be very similar due to similar satellite geometry (El-Rabbany, 2002) and will be negligible if we are not interested in absolute positions. There are many more error sources such as monument noise due to random ground motion (Beavan, 2005), bad satellite geometry and corresponding imprecision in the position estimates ${ }^{1}$ or relativistic effects (Hofmann-Wellenhof et al., 1993), which we cannot discuss here.

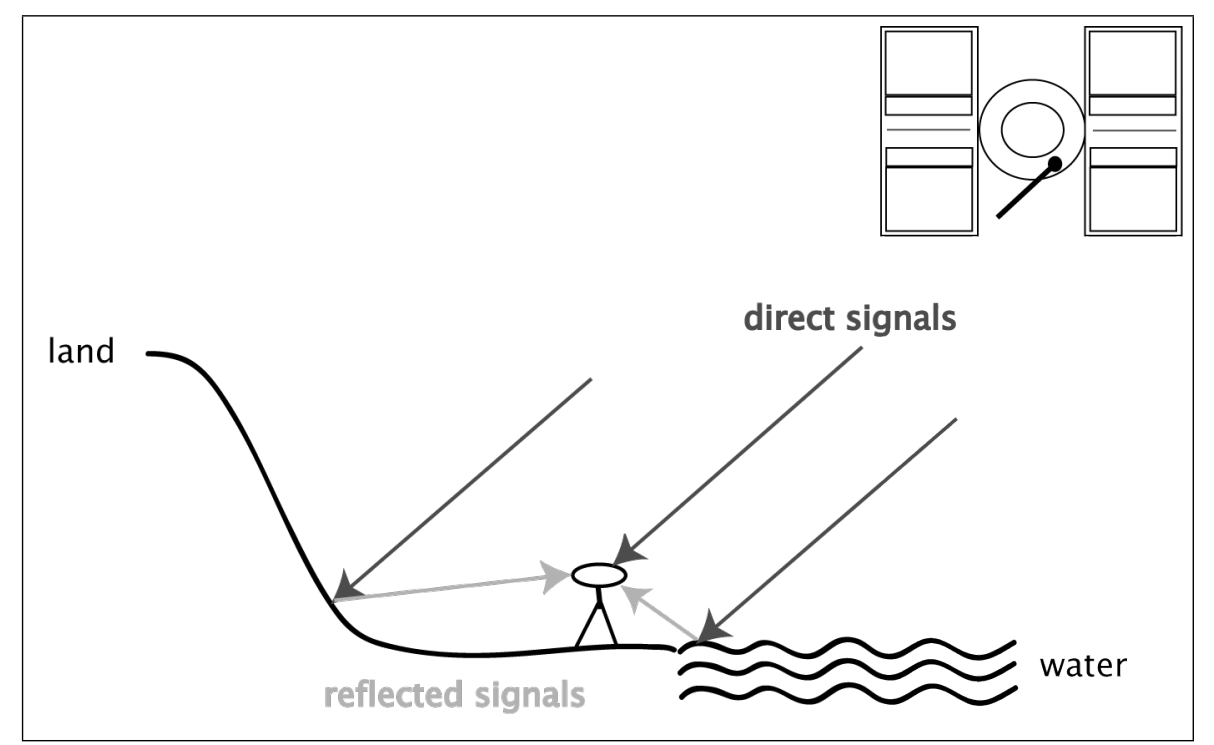

Figure 2.2: Multipathing of GPS signal: Reflectors in the vicinity of the receiver such as water or topography cause additional arrivals (light grey arrows) that distort the direct signal (dark grey arrows) from the satellite.

\footnotetext{
${ }^{1}$ This is technically not an error but a measurement precision problem. As the result, however, is the same (i.e. an uncertainty in the measured position) we chose to mention the effect at this point.
} 


\subsection{Deformation and stress}

As discussed above, ground displacement caused by deformation can occur in different ways and we can measure it for example with GPS. But if a block of material is in equilibrium, no deformation will occur at all. A force per unit area with a certain direction (traction) is necessary to deform a material (Jaeger et al., 2007). Consideration of all force vectors on one point leads to the symmetric 3D stress tensor $\sigma$ :

$$
\sigma=\left(\begin{array}{lll}
\sigma_{11} & \sigma_{12} & \sigma_{13} \\
\sigma_{12} & \sigma_{22} & \sigma_{23} \\
\sigma_{13} & \sigma_{23} & \sigma_{33}
\end{array}\right)
$$

By coordinate transformation this general notation can be rewritten so that only the diagonal elements are non-zero, representing a principal axes system. The diagonal elements are then called principal stresses and are commonly used to describe the state of stress (Means, 1976).

Similarly, deformation is described with the strain tensor $\epsilon$ :

$$
\epsilon=\left(\begin{array}{lll}
\epsilon_{11} & \epsilon_{12} & \epsilon_{13} \\
\epsilon_{12} & \epsilon_{22} & \epsilon_{23} \\
\epsilon_{13} & \epsilon_{23} & \epsilon_{33}
\end{array}\right)
$$

Hooke's law ${ }^{2}$ defines the general relation between stress $\sigma$ and strain $\epsilon$ in elastic media via the fourth-order stiffness tensor $c$ (Means, 1976):

$$
\sigma_{i j}=c_{i j k l} \epsilon_{k l}
$$

The properties of this equation and the stiffness tensor in particular for different cases will be discussed later, when we introduce seismic anisotropy (see section 3.1). For now the fact that the relationship is linear will be sufficient.

According to Jaeger et al. (2007), assuming linear elasticity is a good approximation for most bulk rock masses, since - although the relation between stress and strain is not linear over a wide range of stresses - stress changes are often small, and incremental changes cause approximately linear behaviour in most rocks. In isotropic rocks, the direction of principal stress and principal strain must coincide due to symmetry requirements (Means, 1976; Jaeger et al., 2007). In anisotropic materials, however, the relationship is not straighforward and depends on the elastic properties of the material (Means, 1976). Figure 2.3 illustrates the principle: In general, the symmetry axis of a given, anisotropic material will not coincide with the principal stress directions (see figure 2.3b). Accordingly, the principal strains

\footnotetext{
${ }^{2}$ Indices $i, j, k, l, m, n$ are indices for spatial coordinates; they run from 1 to 3 and use the Einstein summation convention if they occur repeated in a term in all following sections, if not stated otherwise.
} 
will have directions different from both stress directions and symmetry axis (Means, 1976). Only if the symmetry axis of an anisotropic medium coincides with one of the principal stress directions do the principal stresses and strains have the same direction (see figure 2.3a). This relationship often holds for anisotropy considerations, as discussed in section 3.2, but we have to keep in mind the possibility of the general case of different principal axes directions.

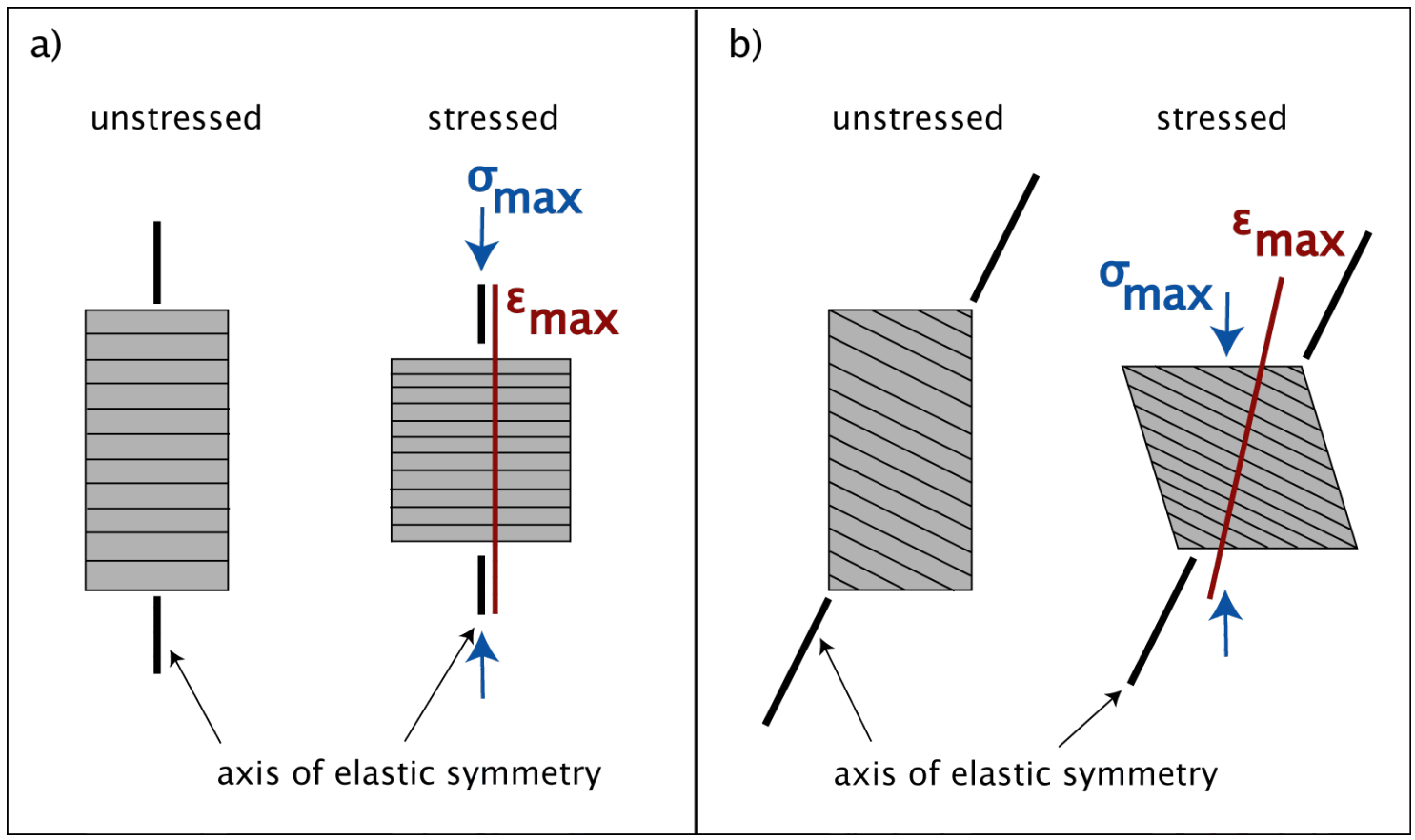

Figure 2.3: Illustration of stress and strain directions in comparison to different symmetry axis orientations. The black arrows point to the axes of symmetry (bold black lines) in both figures, the blue arrows show the direction of maximum compressive stress $\sigma_{\max }$ and the red lines are the directions of maximum compressive strain $\epsilon_{\max }$. a) shows the case of symmetry axis parallel to $\sigma_{\max }$ such as for stress induced microcracks. b) shows the case of symmetry axis at random orientation to $\sigma_{\max }$, e.g. for structurally induced anisotropy. Drawings are schematic only and actual angles will depend on material properties. Figure adapted from Means (1976).

\subsection{Sources of deformation}

The above sections gave an overview about how to measure deformation and the actual forces controlling it. Now we summarise the most important processes that are likely to cause stress and corresponding crustal deformation in the investigated areas. 


\subsubsection{Tectonic deformation}

All three areas investigated in this study are located close to convergent plate boundaries and situated in the respective overriding plates (Sagiya et al., 2000; Wallace et al., 2004). We expect to see long-term deformation on the scale of plate movement $(\mathrm{cm} / \mathrm{yr}-\mathrm{mm} / \mathrm{yr}$, see DeMets et al., 1994). In very general terms, subduction will cause compressional strain accumulation with directions of maximum shortening roughly parallel to the subduction direction, which is at least partly released in subduction interface earthquakes (Savage, 1983). Furthermore, there might be movement away from the subduction trench in the overriding plate due to strain accumulation, or towards the trench if interplate coupling is low and there is contribution from back-arc spreading mechanisms (Sagiya et al., 2000). This very simple scheme can get more complicated in areas where several plate boundaries meet each other (Takayama and Yoshida, 2007), in the presence of other major tectonic boundaries or where slip partitioning on the subduction interface occurs (Loveless and Meade, 2010). Furthermore, back-arc settings can show high extension roughly perpendicular to the subduction trench and high and shear strain rates (Cole et al., 1995). As volcanic activity is often related to subduction zones and the associated back-arc settings such as in the Taupo Volcanic Zone (Wilson et al., 1995), further complicating factors can be introduced into the deformational system.

\subsubsection{Volcanic deformation}

The main source of deformation in volcanic systems is the emplacement or migration of magma or hydrothermal fluids in the crust. According to Segall (2010), three different general and simplified types of magma body have to be considered:

- a spherical or ellipsoidal magma chamber

- magmatic conduits and pipes

- dikes and sills

The surface deformation associated with these types can be approximated with models such as the "Mogi" model of a pressurised point source in a homogeneous, elastic half-space (Mogi, 1958) and others. However, a number of assumptions and approximations go into such models: Viewing the magma body as entirely liquid, conceptualising the surrounding crust as an elastic solid vs. a viscoelastic rheology or assuming homogenous elastic properties are only a few examples of the limitations associated with the modelling of volcano deformation (Segall, 2010). In addition, Cayol and Cornet (1998) have shown a significant influence of volcano topography on the source parameters inferred from surface deformation: For a simple axisymmetric cone volcano with a slope of $30^{\circ}$ the volume change of the source of deformation can be overestimated by up to $50 \%$, if a plane surface is assumed instead of the actual topography. There is a tradeoff between source size and overpressure (McTigue, 
1987) and also the decision between magma or hydrothermal fluids causing the deformation cannot be made solely based upon geodetic observations (Segall, 2010). Other methods such as gravity or electromagnetics are often used to give better constraints. 


\section{Seismic Anisotropy}

Anisotropy in general is the directional dependence of a certain property. In case of seismic anisotropy, the property is the seismic wave velocity. Although the earth is assumed to be isotropic for many applications in seismology, it has been widely recognised that there is anisotropy present in the crust and the mantle (e.g. Crampin and Lovell, 1991; Hess, 1964). The main mechanisms causing seismic anisotropy are alignment of minerals or preferred orientation of cracks (e.g. Crampin and Peacock, 2005; Savage, 1999; Kendall, 1994). There are different types of anisotropy, depending on whether we look at $P$-waves (e.g. phase delay from $P_{n}$-arrivals), $S$-waves (e.g. shear-wave splitting) or surface waves (e.g. surface wave scattering) (Park and Levin, 2002). The following sections explain shear-wave splitting, discuss the possible causes and establish a theoretical connection to stress and strain.

\subsection{What is shear-wave splitting?}

Shear-waves travelling through an anisotropic medium split into two components with fixed, mutually perpendicular polarisation. The polarisations of the components are not necessarily exactly perpendicular to the propagation direction of energy (Savage, 1999), therefore the two components are often referred to as "quasi"$S$-waves. However, as the angle between propagation and polarisation direction is usually not more than $10^{\circ}$ (Savage, 1999) the term "quasi" will be dropped hereafter. The two $S$-waves travel at different velocities (figure 3.1) and the orientation of the fast component (also often referred to as fast polarisation or fast direction) $\phi$ and the time delay $\delta t$ can be measured at the surface.

Babuska and Cara (1991) derive this from the consideration of elastic tensors in anisotropic media and plane wave propagation for the hexagonal symmetry case:

The basis is Hooke's law, as shown in section 2, equation 3. Because the stress as well as the strain tensor are symmetric, both $i j$ and $k l$ are interchangable, respectively. With three diagonal and three independent off-diagonal elements each, this leads to $6 \times 6=36$ independent elements of the fourth-order, material specific stiffness tensor $c_{i j k l}$. Hooke's law is derived making use of the energy density function $U=c_{i j k l} \epsilon_{i j} \epsilon_{k l} / 2$. Its characteristic $\epsilon^{2}$ introduces a further level of symmetry to the stiffness tensor, as the pairs $i j$ and $k l$ become mutually interchangable, too. The 36 elements of $c_{i j k l}$ can formally be arranged into a 6x6-matrix $C_{m n}=c_{i j k l}$ with $m$ and $n$ from 1 to 6 for the different combinations of $i$ and $j$ and $k$ and $l$, respectively. With symmetry in $i j$ and $k l$, this matrix reduces to six diagonal and 30/2=15 independent off-diagonal elements, leaving the stiffness tensor with $15+6=21$ independent elements in the most general anisotropic case.

Hexagonal symmetry can be used to approximate many earth settings: Layering of sediments or metamorphic rocks, or alignment of plane cracks are examples for hexagonal symmetry in the earth. The matrix of elastic coefficients in this case 


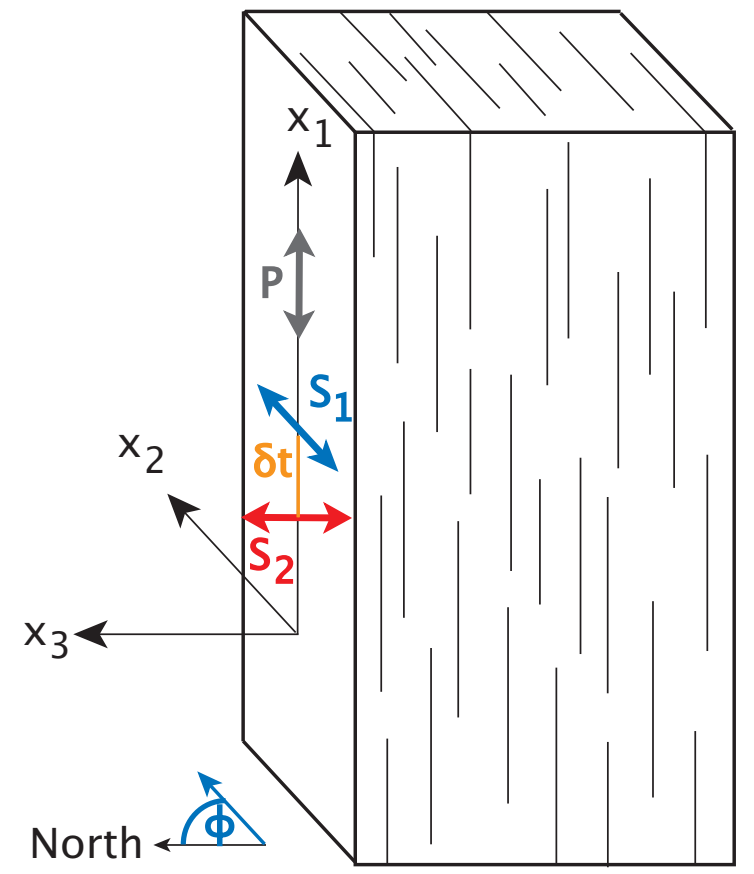

Figure 3.1: Principle of shear-wave splitting for near-vertical plane cracks. $P$ (grey), $S_{1}$ (blue) and $S_{2}$ (red) indicate the polarisation direction of the $P$-wave, the fast and the slow $S$-wave, respectively. The angle $\phi$ (blue) is the measured fast polarisation direction and the delay time $\delta t$ (orange) is the time difference between the fast and the slow shear-wave. Figure after (Babuska and Cara, 1991).

looks as follows:

$$
C_{i j}=\left(\begin{array}{cccccc}
A & A-2 N & F & 0 & 0 & 0 \\
A-2 N & A & F & 0 & 0 & 0 \\
F & F & C & 0 & 0 & 0 \\
0 & 0 & 0 & L & 0 & 0 \\
0 & 0 & 0 & 0 & L & 0 \\
0 & 0 & 0 & 0 & 0 & N
\end{array}\right)
$$

where the $x_{3}$-axis is the axis of symmetry and $A, C, F, L$ and $N$ are the five independent elastic coefficients (as defined by Love in 1927, see Babuska and Cara, 1991 for reference) in a Cartesian coordinate system. For a plane wave propagating in $x_{1}$-direction with the amplitude and polarity vector $\vec{a}$ and the relation to stress and strain, we can derive phase velocity $c$ and the polarisation directions $\vec{a}$ by finding the eigenvalues and eigenvectors of the Christoffel matrix ${ }^{3}$

$$
m_{i j}=\frac{1}{\rho}\left(\begin{array}{ccc}
A & 0 & 0 \\
0 & N & 0 \\
0 & 0 & L
\end{array}\right)
$$

With the three eigenvalues $\lambda_{1}=A / \rho, \lambda_{2}=N / \rho$ and $\lambda_{3}=L / \rho$ and the asssociated eigenvectors

\footnotetext{
${ }^{3}$ see Babuska and Cara (1991) for full derivation of the Christoffel matrix from the wave equation and the stiffness tensor.
} 
$\overrightarrow{a_{1}}=\left(\begin{array}{l}1 \\ 0 \\ 0\end{array}\right), \quad \overrightarrow{a_{2}}=\left(\begin{array}{l}0 \\ 1 \\ 0\end{array}\right), \quad \overrightarrow{a_{3}}=\left(\begin{array}{l}0 \\ 0 \\ 1\end{array}\right)$

we compute three different phase velocities $v_{P}>v_{S 1}>v_{S 2}$ for $A>N>L$ and density $\rho$ :

$\begin{array}{lll}P \text {-wave }(P): & v_{P}=\sqrt{A / \rho} & \text { polarised in } x_{1} \\ \text { fast } S \text {-wave }\left(S_{1}\right): & v_{S 1}=\sqrt{N / \rho} & \text { polarised in } x_{2} \\ \text { slow } S \text {-wave }\left(S_{2}\right): & v_{S 2}=\sqrt{L / \rho} & \text { polarised in } x_{3}\end{array}$

Figure 3.1 illustrates the principle for the example of aligned, near-vertical plane cracks and shows the parameters $\phi$ and $\delta t$ which can be measured at the surface to infer subsurface anisotropy.

Complications arise through the interactions of waves with interfaces or the free suface (e.g. Liu and Crampin, 1990; Nuttli, 1961). In the simplest case of a plane wave travelling through a homogeneous half-space incident at the free surface, Nuttli (1961) shows that only for incidence angles $i$ less than $\sin ^{-1} \frac{v_{S}}{v_{P}}$ (where $v_{S}$ and $v_{P}$ are $S$ - and $P$-wave speeds at the surface, respectively) the incident waves are in phase and the waveform remains undistorted. For a Poisson's ratio of 0.25 this leads to $i \sim 35^{\circ}$, i.e. near-vertical incidence ${ }^{4}$. For higher angles $i$, phase conversions will cause non-linear particle motion and obscure shear-wave splitting measurements. For local and regional events, curved wavefronts have to be considered instead of plane waves: Booth and Crampin (1985) show that applying the shear-wave window criterion introduced by Nuttli (1961) is sufficient also for curved waves, as particle motion only becomes severely non-linear for incidence angles larger than $40^{\circ}$.

In case of one anisotropic layer, $\phi$ will depend on the orientation of anisotropic features (see section 3.2) and $\delta t$ is influenced by the thickness of the anisotropic layer as well as the amount of anisotropy present (e.g. Park and Levin, 2002) along the ray path according to the following equation (Savage, 1999; Crampin, 1985):

$$
\delta t=L *\left(\frac{1}{v_{S 1}}-\frac{1}{v_{S 2}}\right)
$$

where $L$ is the length of the anisotropic part of the ray path and $v_{S 1}$ and $v_{S 2}$ are the velocities of the fast and the slow component, respectively. Crampin (1994) defines shear-wave anisotropy $(S W A)$ in percent as

$$
S W A=\frac{\max \left(v_{S 1}\right)-\min \left(v_{S 2}\right)}{\max \left(v_{S 1}\right)} * 100
$$

Hence shear-wave splitting measurements are not unique; a thick layer with low anisotropy can cause the same splitting parameters as a thin, strongly anisotropic layer. Additional information from seismic tomography, refraction and reflection

\footnotetext{
${ }^{4}$ As discussed later, our analyses revealed a very low ratio $v_{P} / v_{S}$ in one area, and accordingly a slightly wider range for angles of incidence that could be used. Hence, restricting $i$ to less than $35^{\circ}$ will still exclude dubious results.
} 
seismics, knowledge about the geology etc. has to be taken into account to give a valid interpretation of splitting results.

The case of multiple anisotropic layers is even more complicated. Since the fast direction is independent of the incoming polarisation (as long as the incoming polarisation is not parallel or perpendicular to the fast axis of anisotropy, see section 5.1.2), in theory $\phi$ will only yield information about the uppermost anisotropic part along the raypath. For instance a wave passing through two anisotropic layers will split once in the first layer, and each component will split again into two components. In practice, however, in most cases it is not possible to distinguish between arrivals from multiple anisotropic layers as they arrive too closely spaced in time. Although the four components will most likely not be resolvable (Silver and Savage, 1994), information about the anisotropic layers can be obtained from the apparent fast direction $\phi_{a}$ and delay time $\delta t_{a}$.

Teleseismic $S$ - or $S K S$-phases ( $S$-waves converted to $P$-waves at the core-mantleboundary (CMB) and back to $S$-waves) are examples of phases suitable to derive structures from shear-wave splitting. As this study only focusses on limited areas, $S$ arrivals from local and regional earthquakes are used. This should also overcome the general drawback of shear-wave splitting: the low vertical resolution. For example for $S K S$-phases, the observed anisotropy can result from any part of the ray path between the CMB and the surface (Fouch and Rondenay, 2006). For the $S$-phases used here, the possible location of anisotropic features, however, is confined to the uppermost kilometres of the mantle and the crust (see section 6).

\subsection{Causes of shear-wave splitting}

Shear-wave splitting is caused by anisotropic fabric in the earth. However, different mechanisms apply in the crust and the mantle. For splitting studies on teleseismic events anisotropy in both crust and mantle have to be considered, but local and regional earthquakes do not sample the lower mantle. Hence it can be assumed, that the majority of shear-wave splitting is due to anisotropy located in the crust.

In the mantle shear-wave splitting is assumed to be caused by melt-filled inclusions (Ando et al., 1980) or flow-induced alignment of olivine crystals (e.g. Hess, 1964). The fast polarisation aligns with the preferred orientation of the $a$-axes of olivine crystals in the latter case (Savage, 1999). However, there is ongoing debate about the orientation of the direction of the $a$-axes in relation to strain. Factors like the presence of water, high pressure and temperature are found to influence the deformation mechanisms of olivine (e.g. Mainprice et al., 2005; Jung et al., 2008). As this project only examines the crust further discussion of mantle anisotropy is omitted.

The concept of cracks and microcracks causing crustal anisotropy was established several decades ago (e.g. Crampin, 1981). (Crampin and Lovell, 1991) summarise five possible causes of shear-wave splitting in the crust: 
- alignment of crystals,

- anisotropy directly induced by stress,

- lithologic anisotropy, such as aligned anisotropic grains,

- structural anisotropy, such as fine layering, as well as

- anisotropy due to stress-induced preferred orientation of cracks.

All of these features are likely to cause shear-wave splitting on different scales. Although stress-induced crack alignment has been recognised as the main factor causing relatively uniform shear-wave splitting in the upper crust (Crampin and Lovell, 1991; Kaneshima and Ando, 1989), there is ongoing debate about the influence of structural anisotropy. Boness and Zoback (2006); Zinke and Zoback (2000); do Nascimento et al. (2002) and others have reported contributions from structure such as fine layering and bedding or macrofaults to observed shear-wave splitting. With this study, we hope to contribute to improved understanding of the different regimes, structure and stress.

\subsection{Shear-wave splitting in relation to stress and strain}

\subsubsection{Stress}

Applying non-lithostatic stresses to rocks can make intrinsincally isotropic rock effectively anisotropic and can cause shear-wave splitting due to preferred orientation of fluid-filled microcracks and pores (Nur and Simmons, 1969a; Zatsepin and Crampin, 1997) as shown in figure 3.2. For small stresses the principal directions of infinitesimal strain are parallel to the principal stress directions (see section 2.2 and Means, 1976; Jaeger et al., 2007). If this strain causes microfracturing, the cracks will directly reflect stress directions. The orientation of the fast polarisation under such conditions has been shown to align with maximum horizontal compressive stress $\left(S_{H, \max }\right)$ (e.g. Audoine et al., 2000; Boness and Zoback, 2006). However, several studies cannot distinguish between the effect of microcracks and macroscopic structures which are not (or only in part) related to present day stress conditions and which might not be sensitive to short term variations in the stress field. In many areas such as the Appennines in Italy or New Zealand, normal faults are found parallel to $S_{H, \max }$ and hence can contribute to shear-wave splitting observations (Audoine et al., 2000; Pastori et al., 2009). Savage et al. (1990) emphasise the possibly enhanced effect of local macrofaults over widespread microcracks on observed fast directions at Long Valley caldera.

As shear-wave splitting is at least partly influenced by stress, changes in the latter will cause variations in fast direction and/or delay time. Studies in various settings such as Mt. Ruapehu, New Zealand (Gerst, 2003), Mt. Asama, Japan (Savage et al., 2010b) and the Valhall oil reservoir, North Sea (Teanby et al., 2004a) have reported 


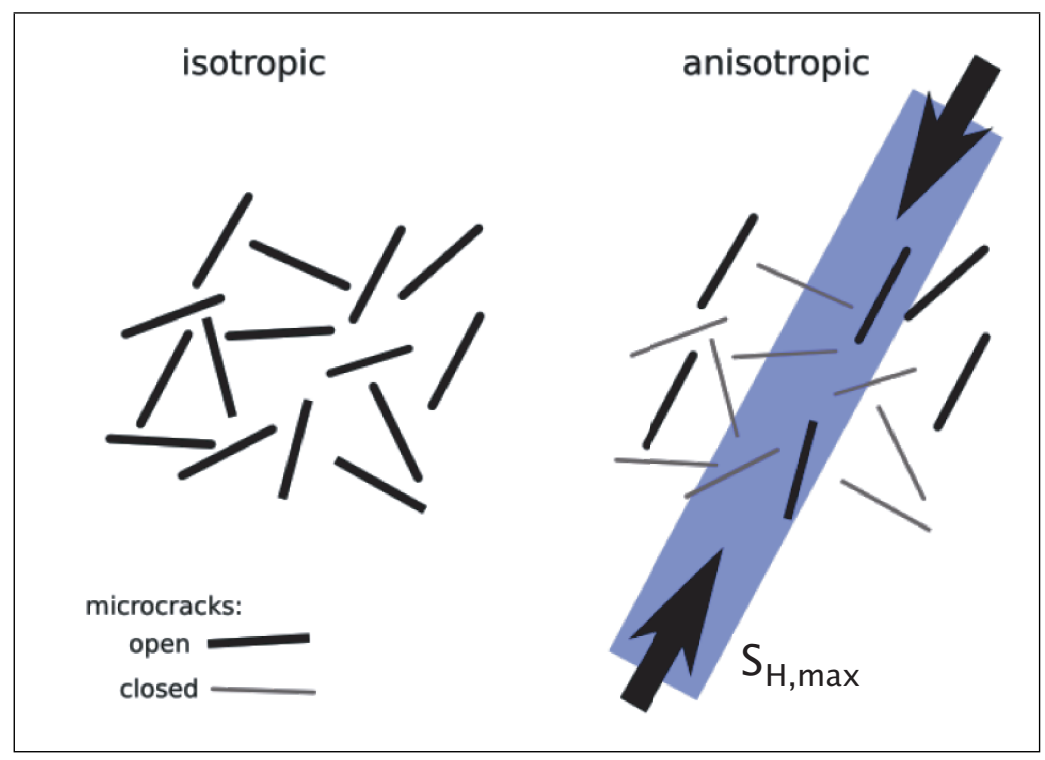

Figure 3.2: Schematic illustration of open and closed cracks due to stress: randomly oriented cracks, all open if no stress is applied. In case of stress application (arrows indicate the direction of maximum horizontal compressive stress, $S_{H, \text { max }}$ ) cracks parallel to the stress direction open, others close. Figure from Wessel (2010).

temporal variations in seismic anisotropy and attributed those changes to varying stresses. Under the assumption that the regional stress is constant over time in most cases, variations in stress might be due to local sources such as oil field exploration or migration of fluids or magma. In either case the limiting factor is differential stress. The regional differential stress has to be small enough to be overcome by local stresses for a change to occur. On the other hand the overall differential stress has to be large enough to cause a deviation of the mean crack aspect ratio from zero in order to make the rock factually anisotropic (Zatsepin and Crampin, 1997).

\subsubsection{Strain}

It has been widely accepted that shear-wave splitting in the mantle is related to strain via lattice preferred orientation of minerals and corresponding anisotropy (e.g. Savage, 1999; Park and Levin, 2002; Long and Silver, 2009). Combined datasets with large scale deformation derived from GPS and splitting of core-refracted phases have been used to determine coupling between crust and mantle by quantifying the (mis-)fit between the mainly crustal surface strain and the splitting fast orientations which link to mantle deformation (Flesch et al., 2005; Lev et al., 2006). The question arises, if strain can be responsible for observed shear-wave splitting in crustal studies, too. It is especially important to assess the case when strain principal axes are not parallel to principal axes of stress, as explained in section 2.

In many applications of crustal shear-wave splitting it can be assumed that 
anisotropy is stress-induced and hence the axis of elastic symmetry is perpendicular to $\sigma_{\max }$ in a material with near-vertical, aligned cracks, as discussed above (see figure 2.3a). The corresponding strain will accordingly have principal axes with the same alignment, i.e. the direction of maximum shortening $\epsilon_{\max }$ parallel to $S_{H, \max }$ (Means, 1976), since if crack alignment is near-vertical it can be inferred that $\sigma_{\max }=S_{H, \max }$. In the case of cracks with an alignment different from near-vertical, $\sigma_{\max }$ will have to be determined from the complete strain tensor. If other controls such as structure make a rock mass intrinsically anisotropic, the axis of elastic symmetry will in general not be aligned with the principal axes of the stress tensor. Consequently the principal axes of the strain tensor will not line up with either the symmetry axes or the principal stress directions (Means, 1976), as shown schematically in figure 2.3b. We want to establish a theoretical framework for the behaviour of shear-wave splitting in such a case with the assumption that strain acts as the major control for shear-wave splitting. $\phi$ will depend on the relative dominance of pure vs. simple shear. For pure shear the direction of maximum compression and extension stay constant throughout the deformation process. Alignment of pre-existing cracks, for example, will be parallel to maximum compression, which is the same for stressinduced alignment. For the case of simple shear we assume that we can look at infinitesimal strain. This theory assumes small strains compared to unity $\left(<10^{-4}\right)$, which is valid especially for processes occurring on time scales much shorter than geological time scales (Jaeger et al., 2007). We can then again infer that cracks will have a preferred orientation perpendicular to the direction of maximum compressive strain $^{5}$.

Hiramatsu et al. (2010, in prep.) also relate shear-wave splitting to strain rate. They find a proportional relationship between normalised delay times of shear-wave splitting and strain rate in central Japan, indicating that not only directions but also the amount of anisotropy depends on the state of strain and the corresponding stresses.

On the other hand, the structural anisotropy which causes the deviation of the symmetry axis from the principal stress directions, by itself could be the major factor influencing shear-wave splitting, as discussed in the previous section.

In the above we have established a framework for how shear-wave splitting should theoretically relate to stress or strain if the two sets of principal axes have different directions or relate to structure. The following sections illustrate how data were collected, analysed and interpreted in different regions to investigate if we can distinguish between the different mechanisms.

\footnotetext{
${ }^{5}$ As often discussed for mantle anisotropy, finite strain theory leads to the conclusion of dry olivine fast axis alignment with the shear direction (Park and Levin, 2002).
} 


\section{Geology and tectonic settings}

\subsection{Taupo Volcanic Zone within the New Zealand plate tectonic framework}

Knowledge of the location of Taupo caldera within the Taupo Volcanic Zone (TVZ) is important to understand the characteristics of the past and current volcanic and tectonic processes. We want to focus particularly on factors influencing deformation and seismic anisotropy to be able to link the observations from the different data types.

At the Hikurangi trench, oceanic crust of the Pacific plate is subducting obliquely under Australian continental lithosphere at a rate of $45 \mathrm{~mm} / \mathrm{yr}$ (DeMets et al., 1994). The TVZ has been recognised as a back-arc area of this subduction margin (e.g. Stern, 1985). It is defined as the active part of the Central Volcanic Region (CVR), an extensional basin with predominantly Quarternary rhyolitic volcanism (figure 4.1) and defined by distinct changes in gravity, heat flow and seismic properties (e.g. Cole et al., 1995; Stern, 1985). It represents the onshore continuation of the Kermadec-Ridge - Havre Trough system (e.g. Wilson et al., 1995).

The TVZ is divided into an old, young and a modern part by Wilson et al. (1995) (see figure 4.1). They define the parts based upon inferred timing of volcanic activity, with $2 \mathrm{Ma}$ through $0.34 \mathrm{Ma}$ for the old and since $0.34 \mathrm{Ma}$ for the young part respectively. The modern part is defined as a fraction of the young zone, with activity during and since the 65 ka eruption of Okataina Volcanic Centre, which is inferred to represent the current state of activity of the TVZ (Wilson et al., 1995). Extension results in opening the TVZ by clockwise rotation since $4 \mathrm{Ma}$ (Wallace et al., 2004; Stern, 1987).

\subsubsection{The Central Volcanic Region}

Within the Central Volcanic Region, surface geology is dominated by Quarternary andesites and rhyolites, with only minor dacites and basalts (Stern, 1985; Wilson et al., 1995). The CVR is flanked by Mesozoic greywacke to the west and the east (Stern, 1985). The modern TVZ itself is dominated by the roughly $\mathrm{N} 40^{\circ} \mathrm{E}$ striking Taupo Fault Belt (e.g Rowland and Sibson, 2001) and eight rhyolitic caldera centres, which are situated in between andesite - dacite strato-volcanoes to the north and the south (Spinks et al., 2005; Wilson et al., 1995). The TVZ is undergoing extension at a rate of $8 \pm 2 \mathrm{~mm} / \mathrm{yr}$ at an orientation of $128 \pm 11^{\circ}$ (roughly perpendicular to the strike of the Taupo Fault Belt; Darby et al., 2000) with predominantly normal dipslip movement (Rowland and Sibson, 2001). A total heat output of $4200 \pm 500 \mathrm{MW}$ is observed from 17 geothermal systems (23 individual geothermal fields) (Bibby et al., 1995).

Drilling at different locations close to and within the CVR yield ambiguous re- 


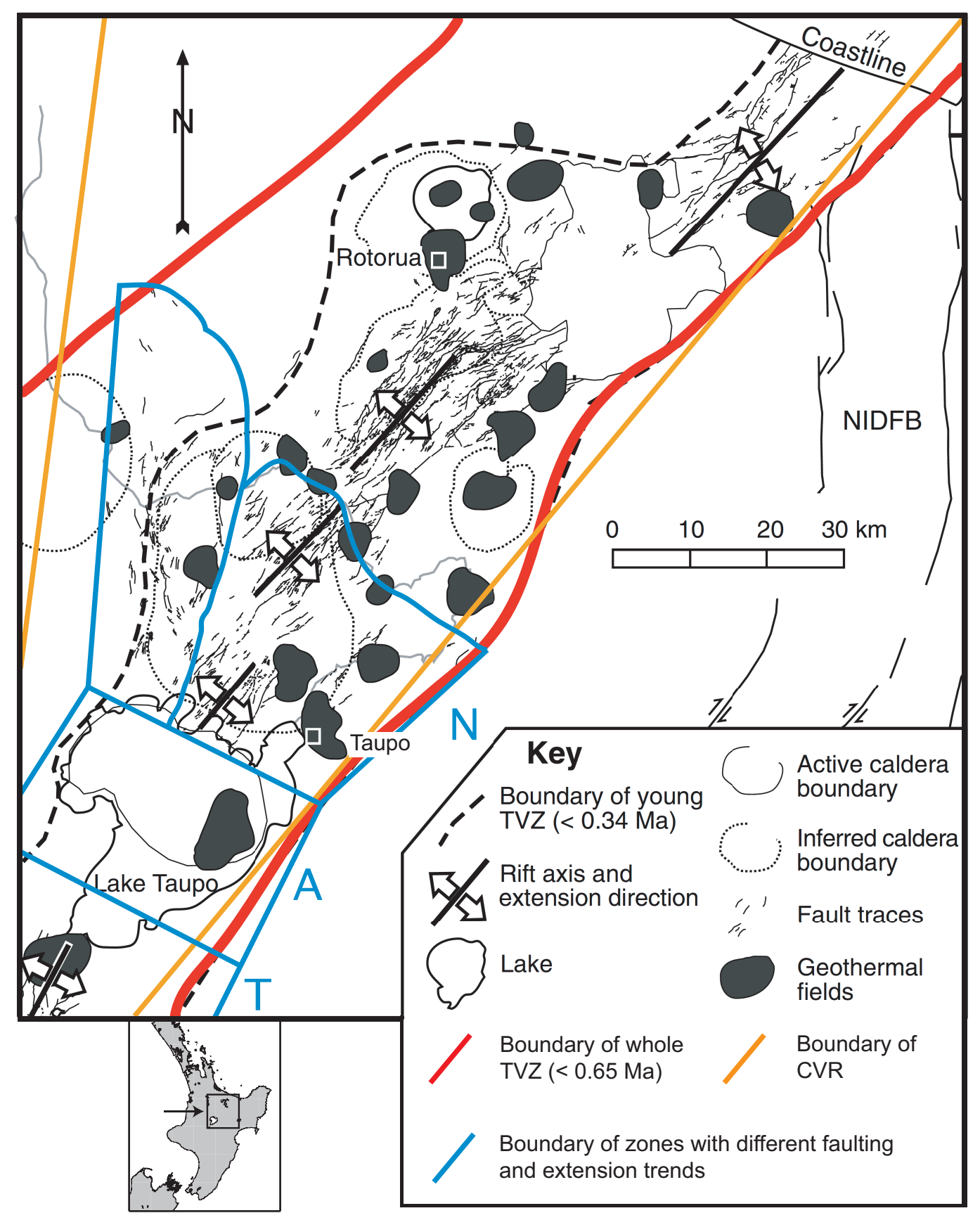

Figure 4.1: Setting of Taupo caldera within the TVZ: The orange line indicates the CVR, the dotted line together with the eastern red line is the young part of the TVZ and the red line shows the whole TVZ. The area between the red line marking the whole TVZ and the dotted line marking the western boundary of the young TVZ is referred to as old TVZ in the text. The modern part just describes individual parts of the young TVZ and is not shown in this plot. The blue zones indicate different segments $\mathrm{N}$ (west and east), $\mathrm{A}$, and $\mathrm{T}$ based on different faulting and extension trends described by Rowland and Sibson (2001). Map in lower left corner shows location of zoom. Figure adapted from Gerst (2003); Sibson and Rowland (2003) and references therein. 
sults: Within a few kilometres of the eastern boundary of the CVR, a basement of Mesozoic greywacke is found at $\sim 5 \mathrm{~km}$ depth (Stern, 1987), confirming the idea of a continuous greywacke basement througout the TVZ introduced by early studies (Shaw and Healy, 1962). Drillholes within the CVR terminate with volcanic and plutonic rocks at 2-3 km and thus neither confirm nor deny the presence of greywacke at depth (Stern, 1985, 1987). It remains unclear if the base of the CVR is made of magmatic rocks throughout the region as suggested by Stern $(1985,1987)$ among others, or if the drill holes were too shallow to touch the greywacke basement. If the basement is indeed continuous throughout the TVZ, it might contribute to observed shear-wave splitting by introducing structural anisotropy: Shaw and Healy (1962) report steeply dipping ${ }^{6}$ (or vertical) contrasting layers of argillite and greywacke with layer thicknesses up to a few metres.

\subsubsection{Taupo caldera}

The caldera itself and its immediate surroundings are governed by rhyolitic volcanic deposits: Large ignimbrite deposits have been reported to show extensive nearvertical macrofracturing (Shaw and Healy, 1962), that could contribute to seismic anisotropy. While active NNE trending faults north and south of Lake Taupo have been mapped and studied extensively (e.g. Rowland and Sibson, 2001; Villamor and Berryman, 2001; Villamor et al., 2007), fault structures within the lake remain obscure due to caldera collapse (Rowland and Sibson, 2001). Mapped faults have been recognised as mostly normal faults with minor strike slip components and this has been confirmed by 19 focal mechanisms in a study by Hurst et al. (2002), giving a more detailed picture than earlier studies that derived strike slip from composite focal mechanisms (see Hurst et al., 2002 for reference). Stress inversion indicates extension dominating in agreement with normal faulting, and the inferred stress tensor with an almost vertical maximum principal stress with a dip of $70^{\circ}$ and the minimum principal stress almost horizontal and with an orientation $148^{\circ}$ from the north (Hurst et al., 2002) is similar to geodetic estimates. From north to south, Rowland and Sibson (2001) divide the area around Taupo caldera in three main parts: the northern part $(\mathrm{N})$ with a dense network of active normal faults striking mainly $\mathrm{N} 45^{\circ} \mathrm{E}$, the lake itself defined by the caldera boundaries which is acting as accommodation zone $(\mathrm{A})$, and the southern termination of the lake reaching towards the active cone volcanoes $(\mathrm{T})$ with normal fault traces striking approximately $\mathrm{N} 27^{\circ} \mathrm{E}$ (see figure 4.1). Similarly, Villamor and Berryman (2006) suggest stepwise changes in extension from north to south and a detailed study of local variations in focal mechanisms indicates changing stresses from north to south (Sherburn et al., 2009). Furthermore, Villamor and Berryman (2001) deduce depth dependent extension rates from fault slip data of $1.9 \mathrm{~mm} / \mathrm{yr}$ on normal faults with an average dip of $75^{\circ}$ close to the surface increasing to up to an average of $6.4 \mathrm{~mm} / \mathrm{yr}$ on faults with a

\footnotetext{
${ }^{6}$ Without further specification of dip angles.
} 
dip of $\sim 60^{\circ}$ at depths of $6-10 \mathrm{~km}$. Additionally, hypocentres of crustal earthquakes in the central TVZ (i.e. north of the lake) have been reported at a maximum of $8 \mathrm{~km}$ (Bryan et al., 1999), whereas they can reach up to $40 \mathrm{~km}$ around the Tongariro Volcanic Centre (Reyners, 2010), further confirming the idea of major changes within the TVZ from north to south. Interestingly, while the NE strike of maximum horizontal stress from Sherburn et al. (2009) agrees with the NE strike of normal faulting north of lake Taupo, $S_{H, \max }$ around the centre and south of the caldera have been reported as $67 \pm 11^{\circ}$ and $120 \pm 12^{\circ}$, respectively (Sherburn et al., 2009), thus deviating from the normal fault strike of $27^{\circ}$ (Rowland and Sibson, 2001).

Variations in strike of normal fault focal mechanisms have been attributed to high pore fluid pressures that can cause pre-existing faults at less ideal orientations to fail even at low differential stresses (Hurst et al., 2002). The presence of fluids is shown by various geothermal areas north and south of the lake (Bibby et al., 1995) and groundwater has been suggested to reside and to be transported at shallow levels in soft ignimbrite and pumice with high porosities as well as in hard ignimbrite with a fracture-related high permeability (Shaw and Healy, 1962).

Volcanic unrest at the caldera has been reported in the last decades: Although there have been no volcanic eruptions, Smith et al. (2007) suggest a possible intrusion of magma at some point before 1986 (i.e. before the beginning of the observed subsidence) at approximately $8 \mathrm{~km}$ depth below the northern shore of the lake and have attributed subsidence observed from lake levelling to fluid release and contraction within the magma body. Furthermore, Jolly et al. (2008) use an inflating point source close to the location from Smith et al. (2007) to explain GPS signals observed at stations around the lake in 2006 - 2008. Our analyses of GPS data, however, cannot extract an umambiguous signal of magma intrusion from this particular GPS signal, as will be discussed later.

\subsection{Kyushu, Japan}

The island of Kyushu is located on the Armurian plate in southwestern Japan and is considered to represent an arc-arc junction between the Southwestern Japan arc to the northeast and the Ryukyu arc to the southwest (Kamata and Kodama, 1994). Oblique convergence of the Philippine Sea plate towards $\mathrm{N} 55^{\circ} \mathrm{W}$ at $62-68 \mathrm{~mm} / \mathrm{yr}$ is accommodated by subduction at the Nankai trough southeast of Kyushu (Miyazaki and Heki, 2001). Figure 4.2 illustrates how the situation is further complicated by westward subduction of the Pacific plate under the North American plate ${ }^{7}$ to the west at a rate of approximately 80-90 mm/yr (DeMets et al., 1994; Townend and Zoback, 2006). The oblique convergence is accommodated by dip slip movement on the subduction interface as well as dextral strike-slip along the Median Tectonic

\footnotetext{
${ }^{7}$ There is ongoing debate about the actual plate and microplate settings in this area, for discussion see Loveless and Meade (e.g. 2010).
} 


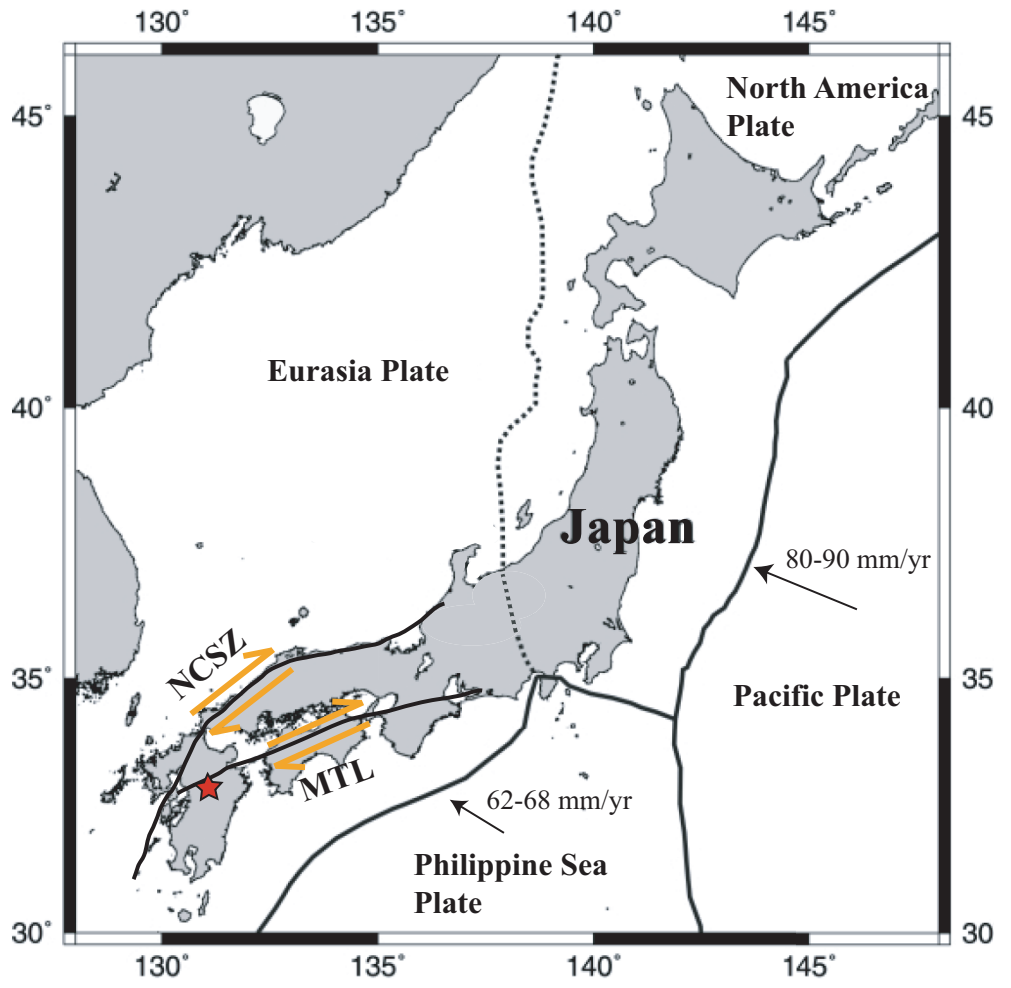

Figure 4.2: $\quad$ Tectonic setting of Kyushu island, Japan. NCSZ, North Chugoku Shear Zone; MTL, Median Tectonic Line. Orange arrows indicate shear movement. Red star denotes location of Aso caldera. Black arrows show plate movement. Figure compiled from Kurashimo and Hirata (2004); Gutscher and Lallemand (1999); Townend and Zoback (2006); DeMets et al. (1994); Miyazaki and Heki (2001).

Line (MTL, Loveless and Meade, 2010), an inland fault zone roughly parallel to the Nankai trough characterised by schists and other intermediate to high metamorphic rocks (Takai et al., 1965, Chapter 11) . In addition, dextral movement is taken up by the North Chugoku Shear Zone (NCSZ) further north (Gutscher and Lallemand, 1999).

\subsubsection{Aso Volcano}

Aso volcano is the largest caldera $(18 \mathrm{~km} \times 25 \mathrm{~km})$ volcano in southwestern Japan and formed through a series of large pyroclastic eruption-cycles, the youngest dated to $\sim 90$ ka (e.g. Kaneko et al., 2007; Hase et al., 2005, and references therein; figure 4.2). Smaller eruptions post 90 ka have formed a series of central cones in the caldera, of which Nakadake crater is the only active one (Miyabuchi et al., 2008). The associated hot crater lake is one of the most prominent features and has been under close surveillance to detect possible eruptions (Terada et al., 2008). Various features that could cause deformation and/or contribute to seismic anisotropy have been discussed extensively in the literature: In the uppermost kilometre below the crater, studies of the shallow electric structure have revealed an extensive hydrothermal system with gas and heat supplied from a deeper magma source (Hase et al., 2005; Kanda et al., 2008), confirming earlier similar suggestions inferred from seismic velocity analyses (Sudo and Kong, 2001). A crack-like conduit at $\sim 2 \mathrm{~km}$ depth 
detected as a source of long-period tremor by Yamamoto et al. (1999) could serve as pathway for fluid and gas from deeper levels to the active crater. A low velocity zone around 5-6 km depth has been interpreted as a magma chamber with at least $10 \%$ melt, a radius of 2-3 km and a volume of $\sim 100 \mathrm{~km}^{3}$ (Sudo and Kong, 2001; Abe et al., 2010). In addition, Abe et al. (2010) studied receiver functions to investigate the crustal structure below the caldera. They suggest the presence of a low velocity layer $\left(v_{S}=2.0-2.4 \mathrm{~km} / \mathrm{s}\right)$ at depths between $10-24 \mathrm{~km}$, coincident with the location of a swarm of deep low frequency earthquakes and a sill-like source of large-scale deformation in the caldera at a depth of $15.5 \mathrm{~km}$ (Abe et al., 2010, and references therein).

The basement of Paleozoic to Mesozoic metamorphic rocks, Cretaceous granites, and Miocene volcanic and plutonic complexes in central Kyushu is overlain by ignimbrites and ash from the four different eruption cycles at Aso (Kaneko et al., 2007; Kamata, 1989). Furthermore, deposits of Kuju volcano to the northeast of Aso caldera and the hydrothermal/magmatic system of Kuju located between the two volcanic centres (Sudo and Matsumoto, 1998; Sudo et al., 1998) might have an impact on the observations in our study. Similarly, active faults accommodating strike slip motion and representing the continuation of the MTL west of the caldera as well as normal faults striking roughly parallel to the direction of maximum horizontal compressive stress $\left(106^{\circ}\right.$, after Townend and Zoback, 2006) to the northwest (Kamata and Kodama, 1994) have to be considered for analyses of strain and shear-wave splitting in this area. Previous studies suggested two major sets of fast directions north of Aso caldera, one striking roughly east-west as expected for north-south extension and the other one approximately perpendicular to the first set (Kaneshima et al., 1989).

\subsection{The Raukumara peninsula}

In contrast to the regions discussed above, there is no active volcanism around Gisborne on the Raukumara peninsula. Yet situated in proximity to the Hikurangi subduction zone, the Raukumara peninsula is experiencing deformation due to subduction of the Pacific plate under the Australian plate (see figure 4.3): Nicol and Wallace (2007) report extension on the order of $1 \mathrm{~mm} / \mathrm{yr}$ in the northern part of the peninsula and a change to contraction at a rate of roughly $1 \mathrm{~mm} / \mathrm{yr}$ further $\mathrm{south}^{8}$. Strain is accommodated partly by earthquakes on the subduction interface (Nicol and Wallace, 2007) and by periodic aseismic thrusting on the subduction interface, that has been identified as slow slip (Schwartz and Rokosky, 2007, and references therein). The slow slip events have been observed with a recurrence interval of two to three years, cause displacement of approximately 20-25 mm over a period of seven

\footnotetext{
${ }^{8}$ The exact rates are derived from GPS/geologic observations and are reported with $0.46 \pm 13 \mathrm{~mm} / \mathrm{yr} / 1.67 \pm 2.8 \mathrm{~mm} / \mathrm{yr}$ and $-0.18 \pm 5 \mathrm{~mm} / \mathrm{yr} /-0.7 \pm 0.4 \mathrm{~mm} / \mathrm{yr}$ for the north and the south, respectively. A negative number indicates contraction (Nicol and Wallace, 2007).
} 


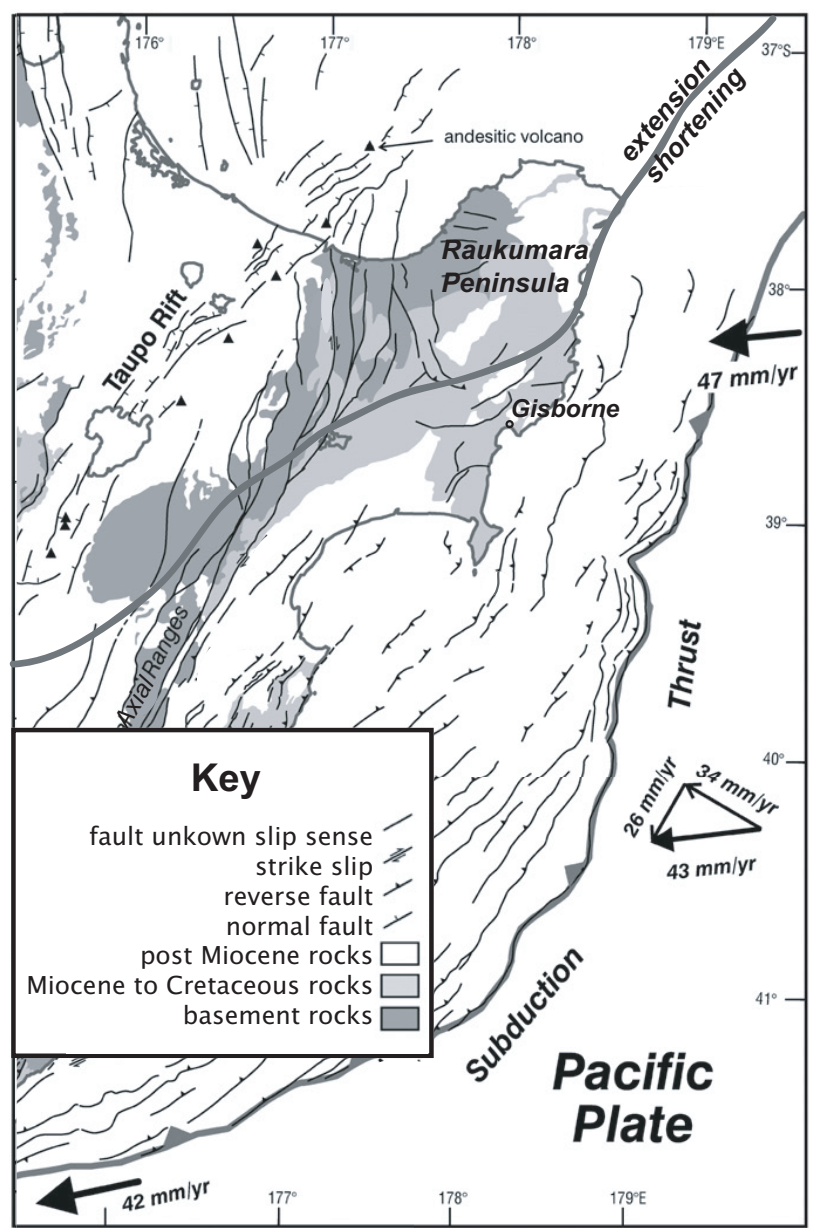

Figure 4.3: Tectonic setting of the Raukumara peninsula. Arrows indicate subduction of the Pacific Plate below the Australian Plate and faults and rock types are shown. The dark grey line going from the SW to the NE corner is separating extension to the west from shortening to the east, after Nicol and Wallace (2007) and mentioned in main body of the text. Figure modified from Nicol and Wallace (2007).

to ten days and happen at relatively shallow depths $(10-14 \mathrm{~km})$ along the plate interface at the transition between aseismic creep and interseismic coupling offshore the Raukumara peninsula (Beavan et al., 2008).

The subduction interface is relatively shallow below the Gisborne area, with a $10^{\circ}$ dip to the west at $15 \mathrm{~km}$ depth (Reyners et al., 1999). Focal mechanisms suggest maximum compressive stress $\sigma_{\max }$ that is trench parallel in the lower plane of the subducting plate and normal to the plate interface in the overriding plate (McGinty et al., 2000). A decrease in crustal thickness from $36-37 \mathrm{~km}$ in the southwest to 17-19 km in the northeast has been observed (Reyners et al., 1999) and uplift of the peninsula has been attributed to sediment subduction and underplating (Upton et al., 2003). The major rock formations are composed of thick sequences of sandand mudstones (Mazengarb and Speden, 2000), so seismic anisotropy is likely to be due to bedding or due to aligned pore spaces and microcracks. 


\section{Shear-wave splitting and GPS processing techniques}

\subsection{Automatic splitting technique}

The increased number of three-component seismometers in the last decades made studies of shear-wave splitting possible in many different settings all over the world (e.g. Audoine et al., 2004; Bianco and Zaccarelli, 2009; Elkibbi and Rial, 2005; Martínez-Arévalo et al., 2003). However, the large amount of data also created the need for an automated algorithm to calculate shear-wave splitting parameters. The following sections describe some developments in this field, which form the basis for the codes used in this study.

\subsubsection{Silver and Chan method}

In 1991, Silver and Chan developed a method to determine shear-wave splitting parameters by correcting the seismograms for anisotropy. The first basic assumption is that phase shifts in the radial component at the free surface are negligible (Silver and Chan, 1991). It has been shown by Nuttli (1961), that this is valid for incidence angles ${ }^{9}$ lower than $\sim 35^{\circ}$ (see section 3.1). The Silver and Chan (1991) method projects the particle motion vector $\hat{p}$ onto the fast and slow directions, $\hat{f}$ and $\hat{s}$, respectively. The original wave can be written as

$$
u(\omega)=w(\omega) \exp \left[-i \omega T_{0}\right] \hat{p}
$$

where $w(\omega)$ is the wavelet function and the complex amplitude of the wave and $T_{0}$ is the time at which the wave arrives at the surface. For a split wave, this can be extended by introducing a splitting operator:

$$
u_{S}(\omega)=w(\omega) \exp \left[-i \omega T_{0}\right] \Gamma(\phi, \delta t) \hat{p}
$$

with

$$
\Gamma=\exp [i \omega \delta T(\phi, \delta t)]=\exp \left[i \omega \frac{\delta t}{2}(\hat{f} \hat{f}-\hat{s} \hat{s})\right]
$$

The seismogram can be corrected for anisotropy by finding the inverse splitting operator $\Gamma^{-1}$ by a grid search over all possible combinations of $\phi$ and $\delta t$. To determine the right combination, the covariance matrix of the particle motion has to be considered: While in the isotropic case the covariance matrix has one nonzero eigenvalue, there are two nonzero eigenvalues $\lambda_{1}$ and $\lambda_{2}$ for anisotropic materials. The combination of $\phi$ and $\delta t$ that results in only one nonzero eigenvalue for the covariance matrix of corrected (and hence linear) particle motion will define the

\footnotetext{
${ }^{9}$ Angle of incidence $i$ is commonly measured as angle from the vertical, i.e. vertical incidence has $i=0^{\circ}$ and horizontal incidence has $i=90^{\circ}$
} 
correct $\Gamma^{-1}$. In the presence of noise, however, $\lambda_{2}$ will never be exactly zero. Hence the method only aims to minimise $\lambda_{2}$. As the value of $\lambda_{2}$ is also a measure for the variance of the noise it can be used to find the $95 \%$ confidence limits around the calculated $\phi$ and $\delta t$.

\subsubsection{Problems and limitations}

S-pick The Silver and Chan method still has some issues of concern. The first subjective part is the $S$-pick. In general, $S$-picks can be generated from travel time calculations for earthquakes with known location and origin time (e.g. with the software TauP, Crotwell et al., 1999). Despite the fact that good velocity models for the whole earth exist (such as ak135, Kennett et al., 1995) and can be incorporated into travel time analyses, $S$-picks especially for local and regional events are still only really reliable when chosen manually. While on the one hand manual selection of the $S$-arrival time can reduce deviations of the real S-phase from the pick due to local velocity anomalies etc., it introduces subjectivity and depends highly on expertise and experience of the user on the other hand.

Null measurements If either the material is isotropic or the initial $S$-polarisation is coincidentally polarised parallel or perpendicular to the anisotropic fast orientation, no splitting is observed (i.e. theoretically any amount of splitting yields 95\% confidence, Savage, 1999). Therefore, if the incoming polarisation is $\alpha$, then measurements that do not fulfill the geometric criterion $20^{\circ}<|\phi-\alpha|<70^{\circ}$ are considered to be nulls (Savage et al., 1996). These results are often characterised by an elongated error surface or a delay time close to the maximum allowed delay time (Levin et al., 2007). They can provide useful information, as for example an area with isotropic earth structure will yield a high percentage of null measurements. In addition, temporal variations in anisotropy should also cause a variation in fast direction of null measurements. Hence, their occurrence should be investigated and ideally be automatically detected by the codes.

Cycle skipping If the frequency content of the $S$-wave is homogeneous over various cycles, cycle skipping can occur. Figure 5.1 illustrates the main principle. One peak of the wavetrain on one component can be matched to two or even more peaks on the other component. Results will hence show various delay times with similarly small $\lambda_{\text {min }}$, with $\delta t$ being multiples of half the dominant period. It can also introduce a $90^{\circ}$ flip of fast direction, as the fast component might be misinterpreted as the slow one if the wrong peaks are matched. This feature can avoided by incorporating a time window before the $S$-arrival, so that fitting the $S$-wave is unambiguous.

Manual splitting window selection The window in which the shear-wave splitting analysis is performed has to be chosen manually in the method by Silver and Chan (1991). Teanby et al. (2004b) emphasise the sensitivity of the shear-wave splitting 


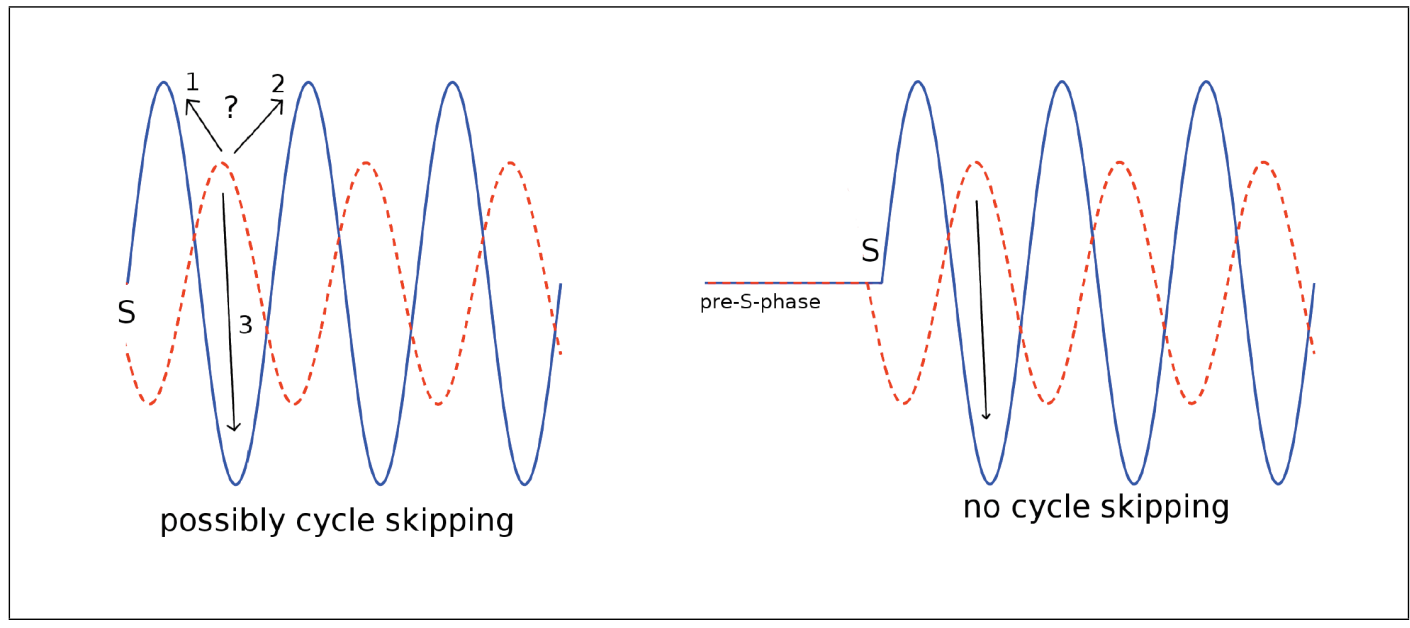

Figure 5.1: Sketch illustrating the principle of cycle skipping. Especially for wavetrains with one dominant frequency, one peak of the one component (red dashed line) can be fitted to two or more peaks in the other component (blue solid line), as can be seen on the left. Including a fraction of the wavetrain before the $S$-arrival (right) avoids this problem. Figure from Wessel (2010).

results to the choice of the calculation window. Automation of this window selection is required to make results more objective and to reduce time or manpower required for processing large datasets.

\subsubsection{Teanby cluster analysis}

Teanby et al. (2004b) attempt to improve the Silver and Chan method by addressing one of the main issues: the necessity of manual shear-wave window selection.

As stated above, the shear-wave analysis window is picked. Ideally it should match the following criteria (e.g. Savage, 1999; Teanby et al., 2004b). It has to

- be long enough to include the $S$-wave onset and multiple periods of the dominant frequency,

- be short enough not to incorporate other phase arrivals and

- ideally start slightly before the $S$-arrival to make the waveform match unambiguous.

The selection of a "good" window will help to prevent cycle skipping and to reduce the influence of noise.

Teanby et al. (2004b) implemented an automation of the process of finding a good shear-wave analysis window. First of all, a number of windows is defined, allowing a range of start and end times. The splitting parameters are then computed for each window. Several different windows will return the same or very similar $\phi$ and $\delta t$; these windows are grouped and the groups are called clusters. Clusters with a 
number of windows less than a defined minimum number are rejected. From the remaining clusters, the best one is chosen based on the within-cluster variance $\sigma_{c}{ }^{2}$ and the mean data variance $\sigma_{d}^{2}$, as shown in figure 5.2. Figure 5.2 (a) shows a cluster with high $\sigma_{c}^{2}$ and low $\sigma_{d}^{2}$ and vice versa in (b). Figure 5.2 (c) has both low $\sigma_{c}^{2}$ and low $\sigma_{d}^{2}$ and is the best cluster overall. From within the best cluster, the window that returns the lowest error is selected as the final shear-wave analysis window. The final results can be visually inspected in diagnostic plots and final (subjective!) decisions about the quality can be made.

(a)

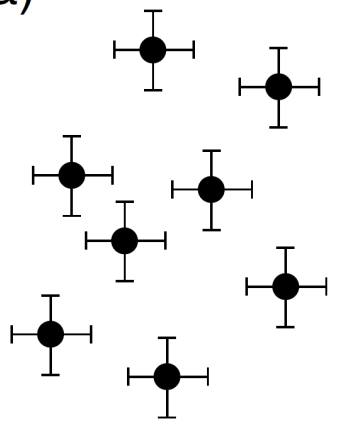

(b)

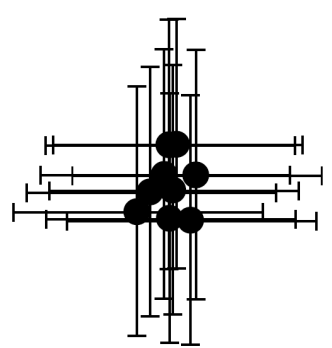

(c)

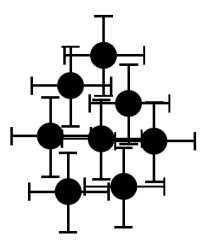

Figure 5.2: Illustration of the difference between within-cluster variance $\sigma_{c}{ }^{2}$ and mean data variance $\sigma_{d}^{2}$. Black dots are points from an arbitrary data set, with error bars in x- and y-direction. While (a) and (b) have high $\sigma_{c}{ }^{2}$ and high $\sigma_{d}^{2}$, respectively, (c) has low values for both types. For further explanation see text. Figure from Teanby et al. (2004b).

This method greatly reduces processing time as all calculations and window selection are done automatically, and in addition increases objectivity and makes the results more reliable and comparable.

\subsubsection{Automated shear-wave splitting}

Wessel (2010) and Savage et al. (2010a) developed an automated technique based on the methods of Teanby et al. (2004b) and Silver and Chan (1991). It further improves objectivity and provides the opportunity of a complete, automatic processing sequence, especially for New Zealand earthquake data from GeoNet ${ }^{10}$.

Earthquake data can automatically be downloaded when the source is the GeoNet website and are converted into a format used for processing. Picks and additional event information such as depth or magnitude are stored in the header of the threecomponent files. The seismograms are then filtered with a set of 14 different, predefined bandpass filters to increase the signal-to-noise ratio and emphasise the actual

${ }^{10}$ http://www.geonet.org.nz 
phase arrivals. The codes contain a minimum signal-to-noise threshold and allow up to three best filters per event to be chosen automatically if the threshold is exceeded. The "best" filters are defined as the ones with the highest value of the product of the signal-to-noise ratio and the bandwidth of the filter in octaves (Savage et al., 2010a). A higher bandwidth is desirable as it maintains variety in the frequency content of waveforms and hence helps to prevent cycle skipping.

The automatic technique organises the seismograms into folders according to the respective seismometer they were recorded on. After this preparation, the files are ready for the actual shear-wave splitting measurement following the techniques discussed above. Main amendments are the introduction of automatic definition of null measurements and with it a set of automatic quality criteria (Wessel, 2010):

- a minimum signal-to-noise ratio of 3 ,

- a cut-off criterion for high delay times, depending on range of allowed delay times,

- fast directions too close or too far from initial polarisation (see definition of nulls above)

- and a maximum error of $25^{\circ}$ in $\phi$.

Up to three results (due to three best filters) are output for each earthquakestation combination. These results are graded to reject measurements with clusters that are too close together, i.e. where the variance of two or more cluster is very similar and hence a decision about the "best" cluster is not straightforward (Savage et al., 2010a). In other words, a "good" cluster should be better than any of the other clusters without ambiguity. Its variance should hence be significantly lower than the variances of all other clusters.

Reference values are the variance varbest of the best cluster chosen by the automatic method by Teanby et al. (2004b) and the difference in $\phi$ and in $\delta$ t compared to the best cluster. An $A$ grade is a result where the variance of any secondary cluster would be $>5 *$ varbest or in other words, where the best cluster is significantly better than all other clusters, as discussed above. To downgrade measurements where secondary clusters give results conflicting with the best cluster, $\phi$ of any cluster must not differ from $\phi$ of the best cluster by more than $45^{\circ} / 2$ and the difference in $\delta \mathrm{t}$ is not more than $0.05 \mathrm{~s}$ for an $A$ grade $^{11}$. A grade $B$ is not as strict: The same criterion applies for the variance as for an $A$ grade, but results are included where $\phi$ differs from $\phi$ of the best cluster between $45^{\circ} / 2$ and $45^{\circ}$. Similarly the difference in $\delta$ t can be up to $0.1 \mathrm{~s}$. All other measurements get $C$ or $D$ grades. As they are not used in this study, discussion of these grading criteria is omitted here.

Furthermore, some measurements might be rejected despite a good grade afterwards, as they turn out to be null measurements (see above).

${ }^{11}$ derived from the parameter tlagmax in the codes which is set to $0.4 \mathrm{~s}$ for measurements of local earthquakes, by taking $1 / 8$ tlagmax 
The results can then be plotted in different ways, which enables the user to manually choose or reject certain measurements afterwards. Figure 5.1.4 shows such a plot.

Figure 5.3: Splitting analysis is made for station KNZ close to Gisborne, event 2217069 in 2004: A) are the three components of the seismogram with $S$-pick as a vertical black line and the splitting window shaded in grey. B) shows the rotated components before and after correction for shear-wave splitting, the $S$-pick in red and the splitting window shaded in grey. C) and D) are the results from the different windows of the cluster analyses with error bars, the blue $\mathrm{x}$ marks the best result. E) is the waveform and $\mathrm{F}$ ) is the particle motion; both uncorrected (left) and corrected (right). Note that the shaded grey area here is the part of the wave which is not within the splitting window anymore. G) is the contour plot of the error of splitting parameters for different values of $\phi$ and $\delta t$, with the blue $\mathrm{x}$ indicating the position of the final result. H) summarises the event information and $\mathrm{J})$ are the splitting results. The corrected seismograms show that most of the energy is successfully removed from the last trace (B) and waveforms fit nicely after correction (E).

\subsection{5. $v_{P} / v_{S}$-ratio calculation}

To improve constraints on elastic parameters and their variations we calculate the ratio of $P$ - to $S$-velocity, if the splitting results show relatively clear variations ${ }^{12}$. Factors such as pore volume, saturation or pore pressure in rocks have been shown repeatedly to affect the effective seismic velocities (e.g. Ito et al., 1979; Chatterjee et al., 1985; Tatham, 1982; Nur and Simmons, 1969b, and references therein). Following the approach by Nur (1972) we calculate an average ratio of $P$ - to $S$ velocity $\left(v_{P} / v_{S}\right)$ along the ray path from the picked arrival times:

$$
\frac{v_{P}}{v_{S}}=\frac{t_{S}}{t_{P}}
$$

with $t_{S}=T_{S}-T_{O}$ and $t_{P}=T_{P}-T_{O}$, where $T_{S}$ and $T_{P}$ are the arrival times of the $S$ - and the $P$-wave, respectively, and $T_{O}$ is the origin time of the earthquake.

\subsection{Positioning processing for different regions}

The raw GPS data have to be processed before we can obtain point positions. The software Bernese V5.0 developed by the Astronomical Institute of the University of Bern is used by GeoNet for all data; in this study this applies to the Gisborne stations. Bernese V4.1 $\beta$ is used for the data from Japan (Sagiya et al., 2000; Miyazaki

\footnotetext{
${ }^{12}$ As explained in later sections we only applied this calculation to Aso caldera.
} 
A) 2004.111.KNZ.2217069.2-6.fb1

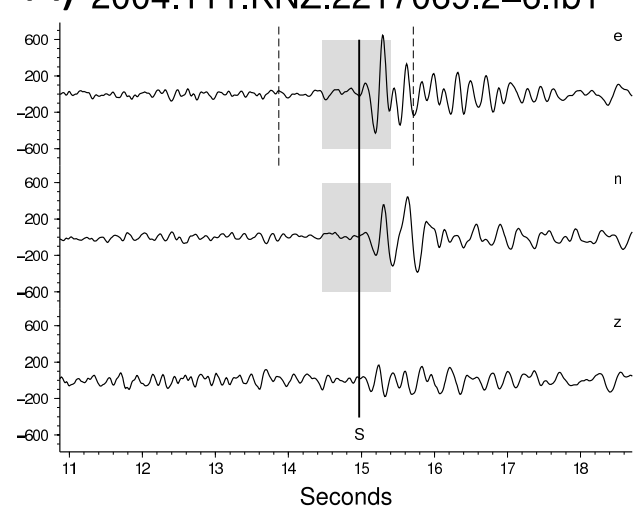

C)

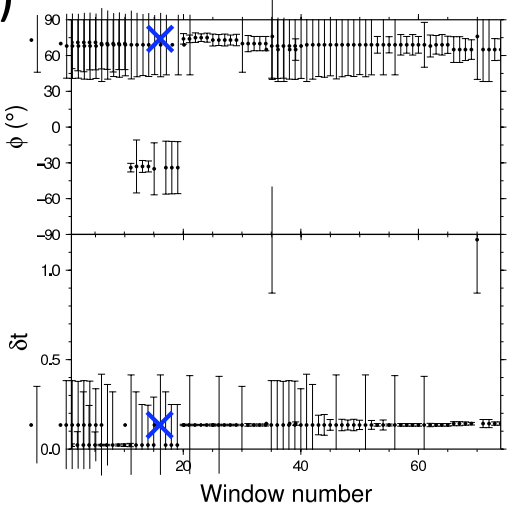

E)

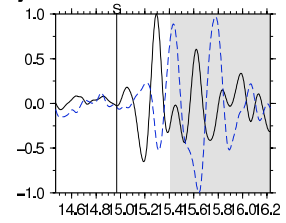

F)
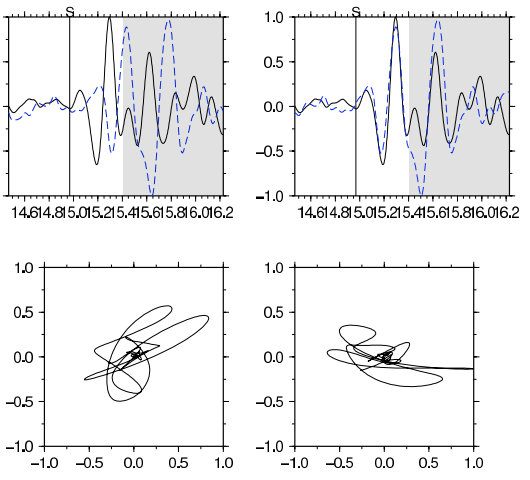

H)

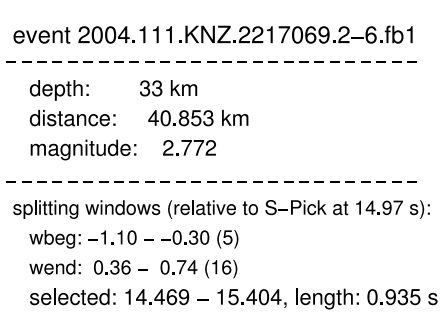

B)

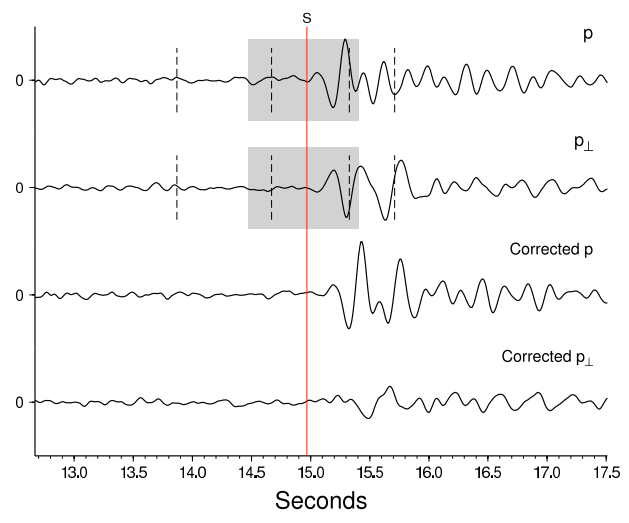

D)

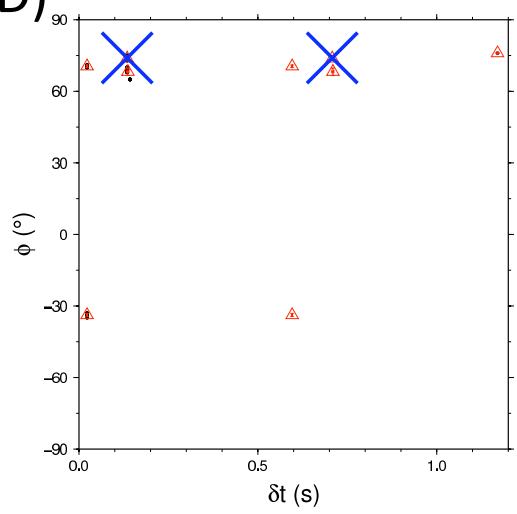

G)

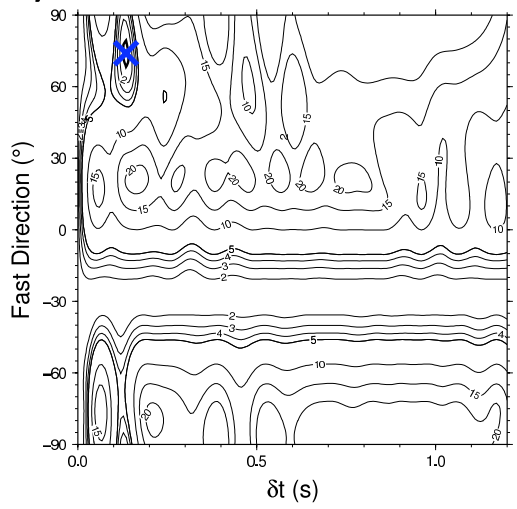

J)

results: GRADE $\mathrm{ACl}$

$$
\begin{aligned}
& \text { fast: } 74.0+/-2.8\left(^{\circ}\right) \\
& \delta t=0.135+/-0.002(\mathrm{~s}) \\
& \text { spol: } 97.6+/-1.3\left(^{\circ}\right) \\
& \text { fast }(\mathrm{abc}): 74.0+/-2.8\left(^{\circ}\right)
\end{aligned}
$$

/Users/home/unglerka/gns_analysis/CWB/gisborne/ 
and Heki, 2001). Taupo data has been processed with GAMIT/GLOBK by N. Fournier (2010, pers.comm.). We will give a brief overview over the processing steps with Bernese V5.0 for the stations around Gisborne, which in principle apply to data from all three regions. More detail can be found in the Bernese V5.0 manual ${ }^{13}$, on the GeoNet website ${ }^{14}$, and in Sagiya et al. (2000).

To make the necessary corrections on the raw data, final satellite orbits and parameters for earth orientation are downloaded from the International GNSS ${ }^{15}$ Service (IGS). They incorporate parameters such as graviational potential from the sun, the moon, the earth and other planets or elastic earth tides. In addition an ocean tide model, a global ionospheric model and patterns for relative antenna phase on the receiver side are included to solve for site displacement in clusters of less than 20 stations. The clustering is necessary to maximise computing efficiency and to keep correct correlations between baselines. In the next step, the clusters are combined and solutions are obtained for the entire (in this case New Zealand) network. In addition, they are aligned to the International Terrestrial Reference Frame (ITRF2000) for the region covering eastern Australia, the south-west Pacific and Antarctica.

GPS data are then simply 3D positions of each GPS station over time. Before we can extract information from the data, obvious outliers and erroneous measurements have to be removed and filtering has to be applied to enhance the signal of interest and reduce noise. The actual parameters will be described in section 6 , as they slightly differ for each region due to different initial data. There are different types of information we use from the processed GPS data, which will be discussed in the following sections.

\subsubsection{Time series}

The position of each station relative to its position at an arbitrarily defined datum versus time can be examined and compared to shear-wave splitting results. Although this method should be a relatively accurate image of the movement of the particular station, the data are basically point measurements. This stands in contrast to the shear-wave splitting result, which give information about anisotropy anywhere along and most likely integrated over the whole raypath of the respective earthquake. It is also possible to detrend the time series to remove long-term tectonic signals. However, since shear-wave splitting might be sensitive to volcanic as well as tectonic and short-term as well as long-term processes, detrending the GPS data implies making a priori assumptions about the nature of the sources of deformation and the origin of shear-wave splitting, which can bias the results. We thus chose to use the whole time series without removing trends.

\footnotetext{
${ }^{13}$ www.bernese.unibe.ch/docs/DOCU50.pdf

${ }^{14}$ http://www.geonet.org.nz/resources/gps/gps-processing-notes.html

${ }^{15}$ Global Navigation Satellite System
} 


\subsubsection{Baselines}

To overcome the limitations of time series, we can look at baseline lengths instead: The distance between two GPS stations will change over time, as the individual station positions change. The magnitude of this change in time is a good proxy for deformation along the imaginary line between the two stations. In addition to depicting processes over a wider area, this way of displaying deformation will automatically have a higher signal-to-noise ratio: Atmospheric noise for example should be very similar on stations sufficiently close together and hence should not affect the distance between the stations. Furthermore, large scale tectonic processes should be similar for stations relatively close together, and hence have only minor effects on the baselines. We calculate the change in baseline length relative to an arbitrarily defined "initial" length, which is the length of the baseline on the first day in our data.

\subsubsection{D strain}

To get a 2D image of deformation, we use the software SSPX (Cardozo and Allmendinger, 2009) to compute strain over the respective areas. The theory behind the software is explained in detail in Cardozo and Allmendinger (2009) and a short summary following Cardozo and Allmendinger (2009) and Means (1976), focussing on the parts and configuration used in this study is given here.

Total displacement $u$ over a given time period at each station is calculated as $u_{i}=x_{i}-X_{i}$, where $x$ is the final position in the deformed state and $X$ is the initial position. Displacement $u$ is related to deformation $G$ and initial position $X$ via equation 12 in the Lagrangian (undeformed) configuration.

$$
u_{i}=t_{i}+G_{i j} X_{j}
$$

where

$$
G_{i j}=\frac{\partial u_{i}}{\partial X_{j}},
$$

i.e. the gradients of the displacements $u$ in the inital state, and $t_{i}$ is an integration constant depending on the coordinate system. Given a number of stations $n$, strain can be computed by solving the resulting system of linear equations as shown below for the 2D case: 


$$
\left[\begin{array}{c}
{ }^{1} u_{1} \\
{ }^{1} u_{2} \\
{ }^{2} u_{1} \\
{ }^{2} u_{2} \\
\cdots \\
\cdots \\
{ }^{n} u_{1} \\
{ }^{n} u_{2}
\end{array}\right]=\left[\begin{array}{cccccc}
1 & 0 & { }^{1} X_{1} & { }^{1} X_{2} & 0 & 0 \\
0 & 1 & 0 & 0 & { }^{1} X_{1} & { }^{1} X_{2} \\
1 & 0 & { }^{2} X_{1} & { }^{2} X_{2} & 0 & 0 \\
0 & 1 & 0 & 0 & { }^{2} X_{1} & { }^{2} X_{2} \\
\cdots & \cdots & \cdots & \cdots & \cdots & \cdots \\
\cdots & \cdots & \ldots & \cdots & \cdots & \cdots \\
1 & 0 & { }^{n} X_{1} & { }^{n} X_{2} & 0 & 0 \\
0 & 1 & 0 & 0 & { }^{n} X_{1} & { }^{n} X_{2}
\end{array}\right]\left[\begin{array}{c}
t_{1} \\
t_{2} \\
G_{11} \\
G_{12} \\
G_{21} \\
G_{22}
\end{array}\right]
$$

The software provides different options of inverting for strain. In this case we chose a nearest neighbour approach. For a given number of nearest neighbours (i.e. observations) at a maximum distance, $G_{i j}$ and $t_{i}$ are inverted for in a user-defined grid from singular value decomposition, solving the above system of the form $b=M a$ as $a=M^{-1} b$, where $b$ is the vector with the known displacements, $M$ is the matrix with the known initial positions and $a$ are the unknown model parameters. For $n$ higher than 3 in the 2D case, the system is overdetermined and the diagonal elements of the covariance matrix represent the variances, i.e. the errors of the model parameters. The Lagrangian strain tensor can then be derived as

$$
E_{i j}=\frac{1}{2}\left[G_{i j}+G_{j i}+G_{k i} G_{k j}\right]=\frac{1}{2}\left[\frac{\partial u_{i}}{\partial X_{j}}+\frac{\partial u_{j}}{\partial X_{i}}+\frac{\partial u_{k}}{\partial X_{i}} \frac{\partial u_{k}}{\partial X_{j}}\right]
$$

In a principal axes system, different values can be obtained from the tensor. As shear-wave splitting fast directions should theoretically align with maximum horizontal compressive strain, we put particular emphasis on directions of $2 \mathrm{D}$ strain shortening axes. However, note that this assumption will only hold if there is no intrinsic, stress unrelated anisotropy (see section 2.2). The actual parameters of strain calculation highly depend on network structure, size etc. and will be discussed individually for each area in section 7 . We want to stress that in this study SSPX is only used to calculate strain, but not strain rates as most other publications in the three different areas (e.g. Beavan and Haines, 2001; Hiramatsu et al., 2010, in prep.). As noted in section 9.3, inverting for strain rates could be done as an additional step in future studies. 


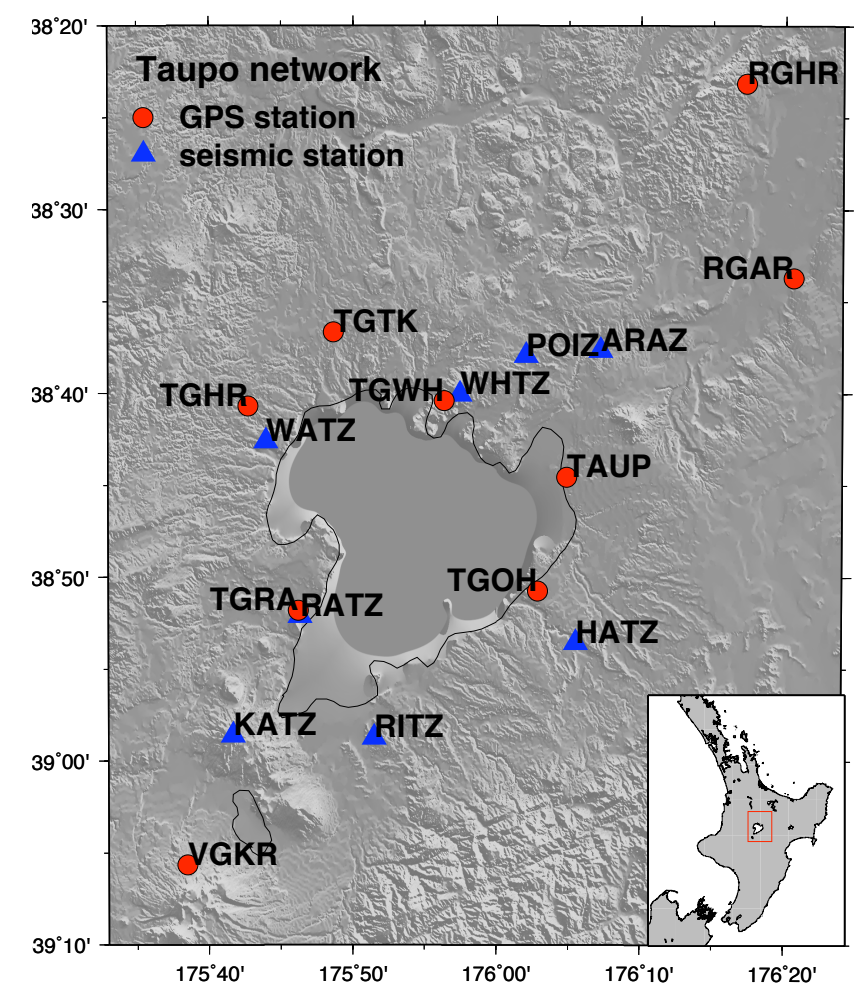

Figure 6.1: Station network around lake Taupo, New Zealand

\section{Data acquisition and processing parameters}

\subsection{Taupo caldera}

\subsubsection{Seismic data}

Eight permanent seismic stations from the New Zealand national network GeoNet ${ }^{16}$ in the vicinity of lake Taupo were chosen for this study (figure 6.1). All but one station are equipped with Sercel L4C-3D broadband, three component sensors. Station RATZ had a Güralp CMG-40T-60S sensor installed in 2008. The specifications and recording times of the eight stations are summarised in table 6.1.

${ }^{16}$ http://www.geonet.org.nz 


\begin{tabular}{|c|c|c|c|c|c|c|c|c|c|}
\hline 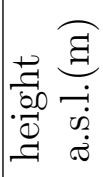 & $\underset{\sim}{\stackrel{\mathcal{F}}{4}}$ & 10 & $\begin{array}{l}\tilde{N} \\
\sim \\
\sim\end{array}$ & $\begin{array}{l}\infty \\
0 \\
10\end{array}$ & $\begin{array}{l}02 \\
10 \\
6\end{array}$ & i⿱ & $\begin{array}{l}\stackrel{2}{2} \\
\stackrel{\sim}{2}\end{array}$ & $\begin{array}{l}8 \\
8 \\
\llcorner 0\end{array}$ & 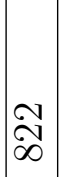 \\
\hline $\mid$ & $\mid \begin{array}{l}0 \\
8 \\
0 \\
0 \\
-1 \\
0 \\
0 \\
1 \\
1 \\
1\end{array}$ & 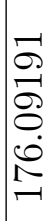 & $\begin{array}{l}10 \\
0 \\
0+1 \\
8 \\
0 \\
10 \\
10 \\
-1\end{array}$ & 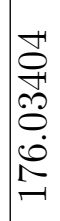 & $\begin{array}{l}2 \\
\Omega \\
1 \\
1 \\
10 \\
1 \\
1 \\
-1\end{array}$ & 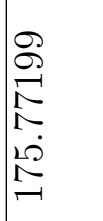 & 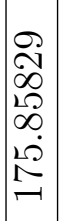 & 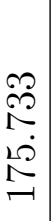 & 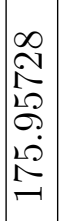 \\
\hline 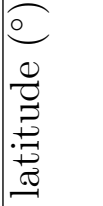 & $\mid \begin{array}{c}0 \\
0 \\
N \\
1 \\
0 \\
0 \\
\infty \\
\infty \\
i \\
1\end{array}$ & 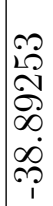 & $\begin{array}{l}0 \\
0 \\
0 \\
1 \\
0 \\
\infty \\
0 \\
1 \\
1\end{array}$ & 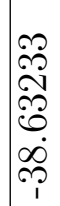 & 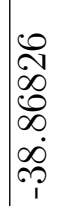 & $\begin{array}{l}0 \\
\sim \\
\infty \\
\infty \\
\infty \\
\infty \\
\infty \\
\sim \\
i\end{array}$ & 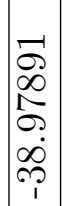 & $\begin{array}{l}\hat{O} \\
\mathscr{D} \\
\dot{\infty} \\
\dot{\infty} \\
\dot{1}\end{array}$ & $\mid \begin{array}{c}\mathfrak{N} \\
\mathbb{1} \\
1 \\
0 \\
0 \\
\infty \\
0 \\
0 \\
1 \\
1\end{array}$ \\
\hline $\begin{array}{l}80 \\
0 \\
0 \\
0 \\
80\end{array}$ & 㐫 & 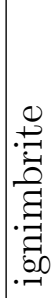 & 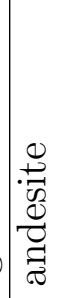 & 㐫 & 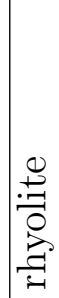 & 竧 & $\mid \begin{array}{l}\dot{\zeta} \\
z \\
z\end{array}$ & 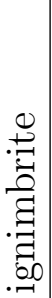 & 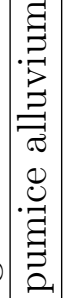 \\
\hline 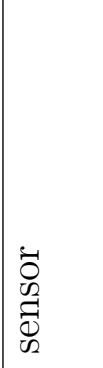 & 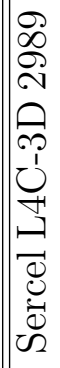 & 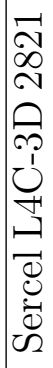 & 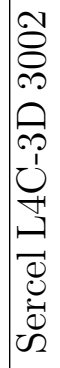 & 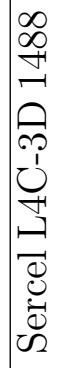 & 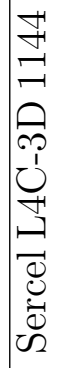 & 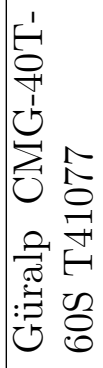 & 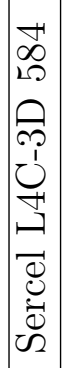 & 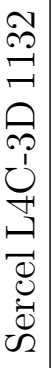 & 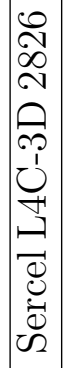 \\
\hline . & 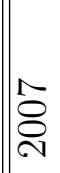 & 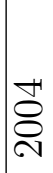 & \&̊) & & 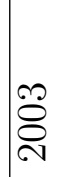 & 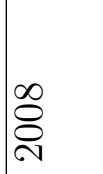 & $\underset{\sim}{\stackrel{\leftrightarrow}{\Xi}}$ & & $\mid \begin{array}{l}m \\
\stackrel{\tilde{D}}{\tilde{N}}\end{array}$ \\
\hline 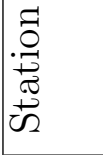 & 亝 & 令 & 㡵 & $\begin{array}{l}N \\
0 \\
2\end{array}$ & 点 & 会 & $\begin{array}{l}\mathrm{N} \\
E \\
\underline{\underline{n}}\end{array}$ & 点 & 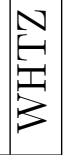 \\
\hline
\end{tabular}

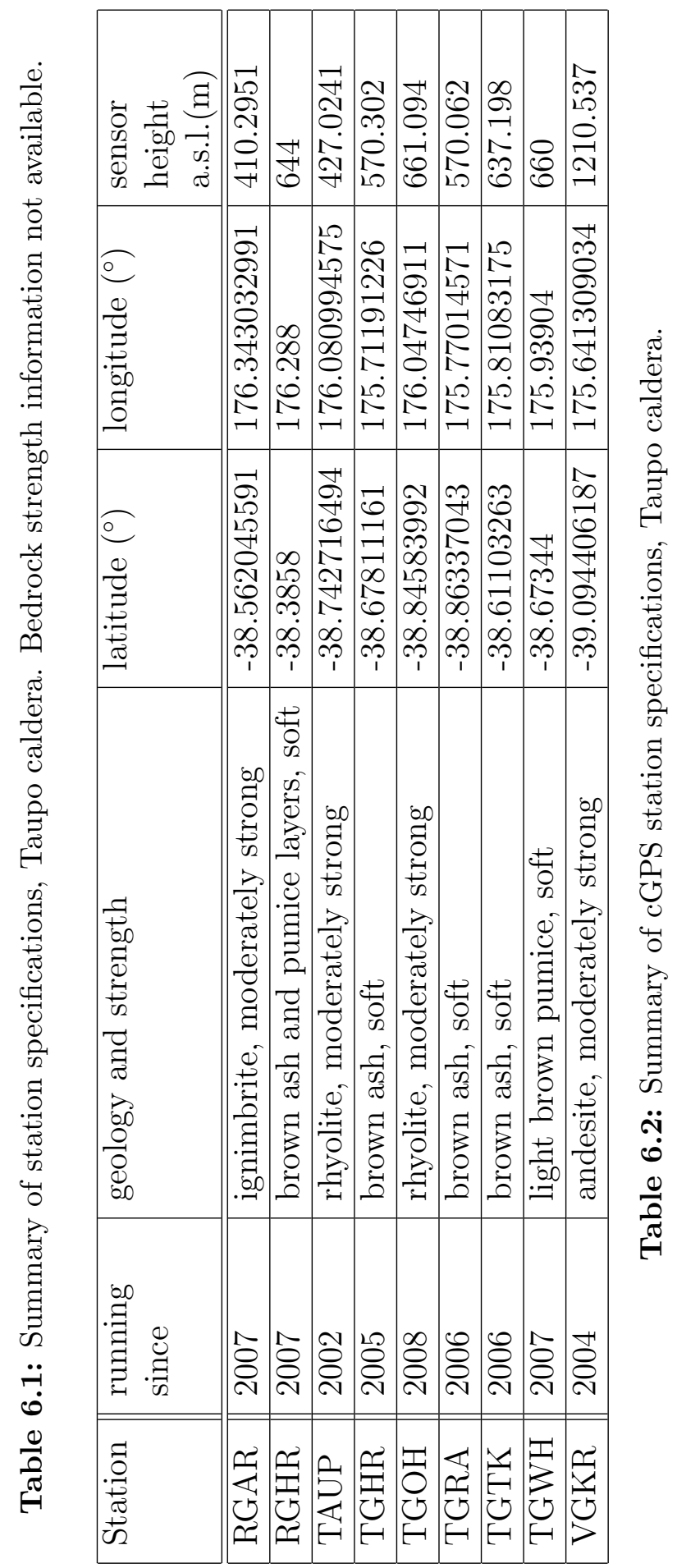


Data files from earthquakes between $-36^{\circ}$ and $-41^{\circ}$ and from $172^{\circ}$ to $179^{\circ}$ that occurred between 2006-2008 were downloaded from the GeoNet ftp-server ${ }^{17}$. After preliminary analyses revealed that a minimum magnitude criterion of $M_{L}=3.0$ for the events does not provide enough reliable splitting measurements (Unglert et al., 2009) the magnitude threshold was lowered to include earthquakes with magnitudes down to $M_{L}=2.0$. According to Gutenberg and Richter decreasing the magnitude by one should ten-fold the number of earthquakes and hence increase the number of shear-wave splitting measurements. However, for the New Zealand GeoNet earthquake catalogue the inferred completeness magnitude is 2.5 (Jacobs et al., 2009), which will consequently decrease the number of earthquakes below that threshold in the catalogue compared to the expected value from the Gutenberg-Richter-law. Moreover, for smaller earthquakes the signal-to-noise ratio will generally be smaller and hence result in fewer splitting measurements for small earthquakes than for larger earthquakes. For lake Taupo there are approximately three times more results from earthquakes between magnitudes 2.0 and 3.0 compared to events with $3.0 \leq M_{L} \geq 4.0$.

Picking In all 17890 earthquakes fitting the above criteria from 2006-2008 were investigated for shear-wave splitting. After downloading the event data, the seismograms were organised according to the station on which they were recorded. Header information like origin time, depth, magnitude etc. was stored and the events were checked for $S$-picks. Seismograms that already had picks in them ${ }^{18}$ are from now on referred to as "catalogue" events, whereas the ones that were picked by hand afterwards are called "manual" events.

While stations HATZ or RATZ, for instance, showed sufficiently clean waveforms to define clear $S$-arrivals (figure 6.2a), the stations in the North Eastern part of the lake, ARAZ, POIZ and WHTZ were not as good (see figure 6.2b). The records proved to be noisy, so that manual picking of $S$-arrivals was difficult or even impossible many times. We used the tool TauP (Crotwell et al., 1999) to calculate theoretical $P$ - and $S$-arrivals from the global 1D earth model for seismic velocities ak135 (Kennett et al., 1995) to have an initial idea were the arrivals should be approximately, which slightly simplified deciding on the time of the $S$-arrival.

\footnotetext{
${ }^{17}$ http://cusp.ftp.geonet.org.nz

${ }^{18}$ Larger events are usually picked by GeoNet.
} 

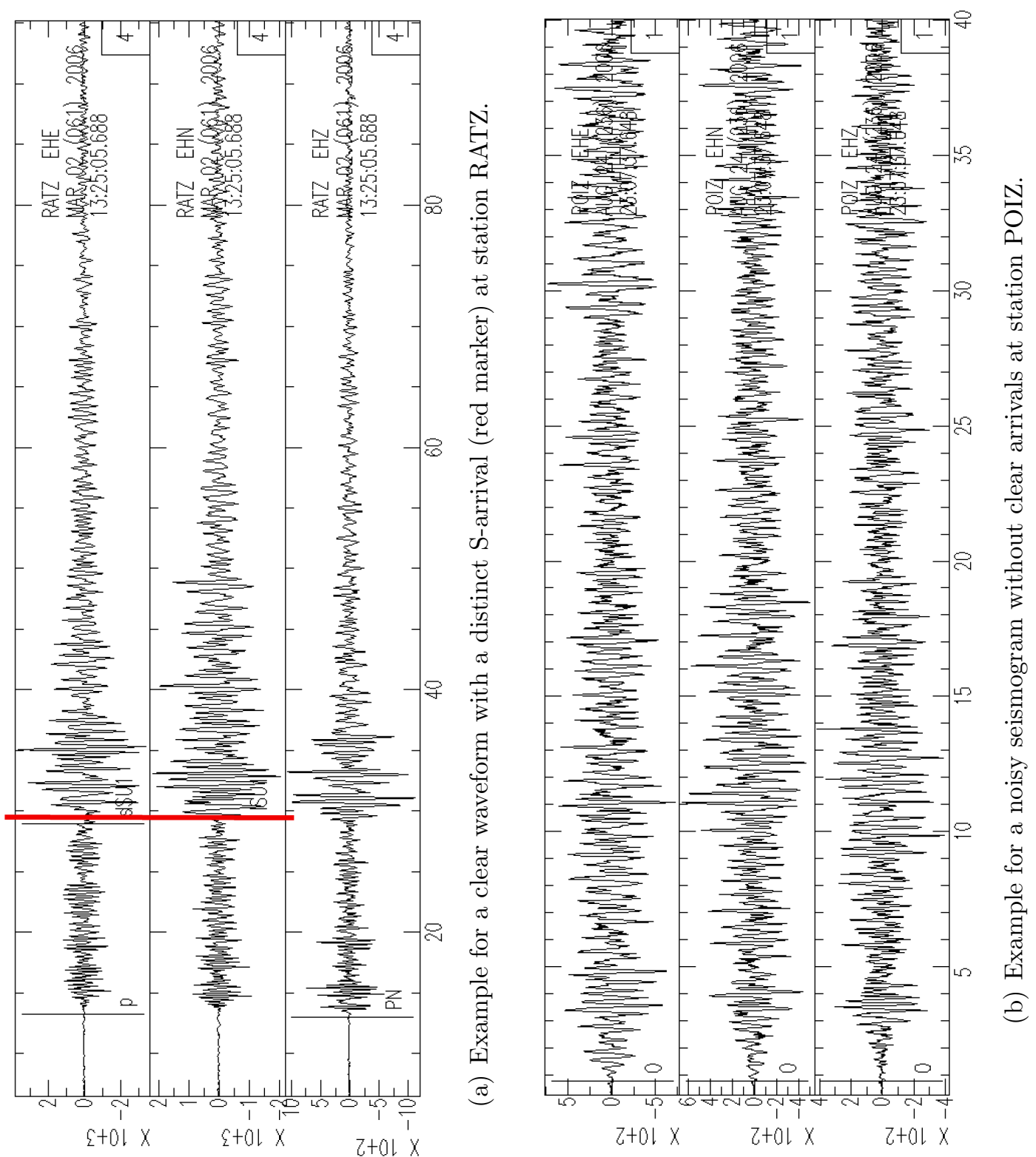

空离 论

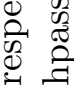
$\rightarrow .07$ : : 포 . .8 엉 렬 की 吾 की 렳 1 . ๘ $\circ \stackrel{0}{0}$ ఫี ฮี ठే क i 之 人 2. ำ 焉

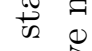

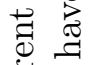
幽䨌 $\because \tilde{\theta}$ 范 串起 굼 용 을 s.. o है 蛹.

ง.

0.7 
Filtering and splitting measurement After this preparation, the seismograms are cut down to a length of $30 \mathrm{~s}$, with the $S$-phase in the centre at $15 \mathrm{~s}$. As the $S$ wave is the desired part of the seismogram, noise, $P$-coda and other phase arrivals have to be supressed. Therefore a set of 14 two-pole one-pass Butterworth bandpass filters is applied. The three best ones are chosen based upon the highest signalto-noise ratio bandwidth product (Savage et al., 2010a), as discussed in section 5.1. Even if a $S$-phase was picked before, in many cases the signal-to-noise ratio of seismograms especially from the northeastern stations (ARAZ, POIZ, WHTZ) was too low and they were thrown out at this point. As a result there are none or only few measurements in certain time spans for these particular stations.

The filtered seismograms were used to obtain splitting measurements using the algorithm developed by Silver and Chan (1991) and the cluster analysis (Teanby et al., 2004b) implemented in the automatic technique by Savage et al. (2010a) as described in section 5.1.

\subsubsection{GPS data}

Six continuous GPS stations around lake Taupo have been chosen for deformation analyses (see table 6.2). Furthermore, one sensor to the south of the lake in the vicinity of Tongariro and two stations northeast of Taupo have been included as a reference. Their locations are plotted in figure 6.1.

Processing Mostly relative and not absolute station positions are important for this study. Data from the nine stations from 2006 through 2008 were already processed by Nicolas Fournier with the GAMIT/GLOBK processing package (2010, pers.comm.) and in format of displacement relative to the position at the first day of the period between 2006 and 2008 at each station. Unfortunately due to an unresolved issue in the processing sequence, the two stations TGHR and RGHR could not be included in the analyses. For the remaining stations, we removed outliers with a standard deviation higher than $0.011 \mathrm{~m}$. This value was chosen somewhat arbitrarily, based upon clear, single outliers which were identified visually. The remaining data have a mean standard deviation of $0.002 \mathrm{~m}, 0.002 \mathrm{~m}$ and $0.009 \mathrm{~m}$ on north, east and vertical components, respectively.

To define an appropriate filter to detect changes on the same time scale as we could expect to see in shear-wave splitting, we had to investigate shear-wave splitting results first. Figure 6.3 shows the density of splitting measurements. We focus on the five "good" stations, i.e. the ones with a relatively high splitting measurement density. The maximum gaps at these stations are on the order of one or two months; the average gap is usually much shorter. Thus, the seismic data in theory enable us to resolve changes in shear-wave splitting parameters on timescales of four to eight weeks and even less at times with high data density. To make the GPS consistent with the whole splitting timeseries, the displacement data were lowpass-filtered with 


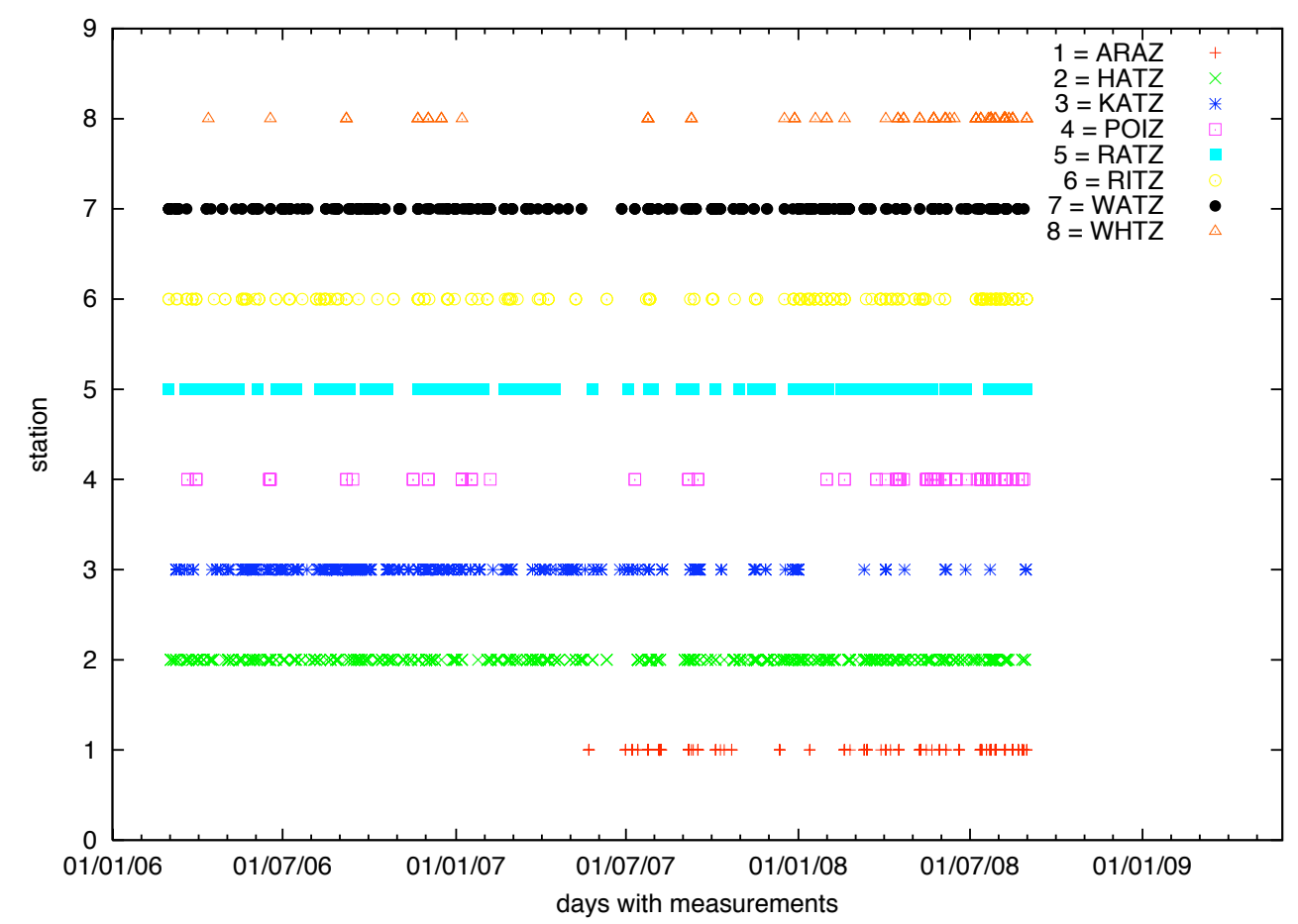

Figure 6.3: Days with shear-wave splitting measurements at the different stations around lake Taupo.

a highcut frequency of $15^{-1}$ days $^{-1}$ to suppress variations on timescales shorter than two weeks. Additionally, a linear interpolation was applied to fill data gaps.

Definition of deformation episodes By visual inspection of the raw and filtered displacement data, seven periods were defined (see table 6.3 and figure 6.4). They were chosen to distinguish episodic deformation and times of relative stability at each individual station but also to be the same for all stations, as we mainly want to identify a common signal. The shear-wave splitting results were then analysed according to these periods.

\begin{tabular}{|l||l|l|l|}
\hline Period & time span (DOY, YYYY) & shear-wave splitting & GPS time series \\
\hline \hline I & $32-121,2006$ & high variations & mostly stable \\
\hline II & $121-227,2006$ & high variations & slow transition \\
\hline III & $227,2006-222,2007$ & high variations & $\begin{array}{l}\text { relatively stable } \\
\text { at most stations }\end{array}$ \\
\hline IV & $222-314,2007$ & high variations & fast change \\
\hline V & $314,2007-32,2008$ & high variations & fast change \\
\hline VI & $32-207,2008$ & high variations & slow change \\
\hline VII & $207-244,2008$ & high variations & stable \\
\hline
\end{tabular}

Table 6.3: Definition of episodes for Taupo caldera, based on GPS timeseries. 

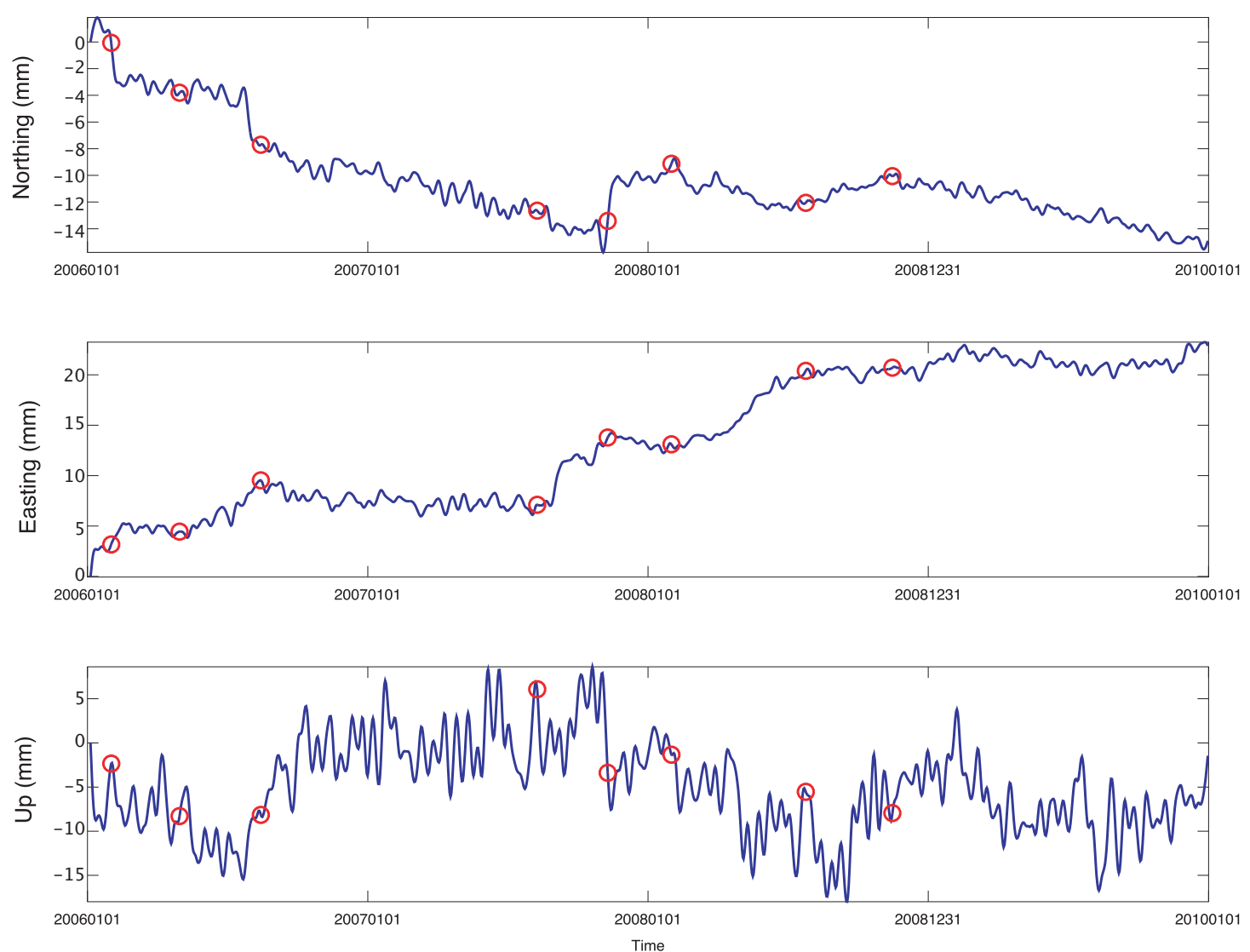

Figure 6.4: GPS time series for station TAUP, filtered with a $1 / 15$ days $^{-1}$ lowpass filter. Blue lines are filtered GPS timeseries, red circles indicate break points for different time periods.

Parameters for strain calculation To calculate strain for the distinct time spans, we used the software SSPX (Cardozo and Allmendinger, 2009), as described in section 5.2.3. Although all three components are used to calculate strain, we keep in mind the high errors on the vertical component and hence only look at 2D strain instead of 3D. Out of the seven time periods derived from displacement, only six have three or more GPS sensors and are hence suitable for strain calculation.

We used the nearest neigbour algorithm to compute strain in the undeformed (Eulerian) configuration from our displacement data. The parameters used for the calculation were chosen after testing different combinations by a trial and error principle based upon certain assumptions:

- The grid cell size was chosen to be $9.5 \mathrm{~km}$. The value has to be relatively large, because with the low station density variations on small scales cannot 
be detected accurately. Yet, the cell size has to be sufficiently small in order to prevent the combination of two stations in one grid cell and the associated loss of information in the big picture.

- The number of nearest neigbours was chosen to be four, as this was the smallest value that still imaged local variations in the strain field while not using too many (or all) stations for each grid cell.

- The maximum station distance was fixed somewhat arbitrarily to $38 \mathrm{~km}$, equal to four times the grid cell size. Testing of a range of values revealed that $38 \mathrm{~km}$ was suitable to show local variations and to give significant results for the area of interest.

It has to be stressed, that this software is usually used for strain computation in networks with hundreds or thousands of stations (Cardozo and Allmendinger, 2009, and references therein). Appropriate care has to be taken when results from such a low density, low station number network as in this study are interpreted. Errors are likely to be high due to high errors in the data, errors introduced due to filtering and the low number of stations.

\subsection{Aso caldera}

\subsubsection{Seismic data}

Seismograms from the area around Aso volcano were kindly provided to M. Savage by her Japanese colleague Takahiro Ohkura from Aso Volcanological Laboratory, Kyoto University. 1272 local events that occurred between $30.5^{\circ}-34.5^{\circ} \mathrm{N}$ and $129.5^{\circ}$ $133.0^{\circ} \mathrm{W}$ with no magnitude limitation recorded on 42 stations $^{19}$ between 2001 and 2008 were processed with the same shear-wave splitting method as the events around lake Taupo. Only events with $S$-picks in them were used, as these data were initially intended for a small pilot study. We chose seven stations with sufficient data for further examination (see figure 6.5 and table 6.4).

Savage et al. (2008) detected a significant change in fast direction at the two stations TAT and MGR, as seen in figure 6.6 for station TAT. Preliminary analyses however could not rule out a change in earthquake location and a corresponding change in $\phi$. A cluster east of TAT was hence used initially to confirm an actual temporal change. Waveforms from this cluster showed a change at the same time as the mean shear-wave splitting fast directions around this station but locations stayed stable. Furthermore, results from all events in that period of time showed the same $\mathrm{NE}$ fast orientation regardless of earthquake locations. It was hence inferred that the change in $\phi$ is a real temporal variation. Accordingly, we investigated results from several earthquakes clusters (ie. events occurring in close locations) to avoid

\footnotetext{
${ }^{19}$ see figure 6.5 for the seven stations that will be used later in this study
} 


\begin{tabular}{|l||l|l|l|}
\hline station & latitude $\left(^{\circ}\right)$ & longitude $\left(^{\circ}\right)$ & altitude $(\mathrm{m})$ \\
\hline \hline HDK & 32.78783 & 130.90867 & 480 \\
\hline MAK & 32.91733 & 130.928 & 380 \\
\hline MGR & 32.79633 & 131.09300 & 700 \\
\hline MKN & 33.00917 & 131.19867 & 670 \\
\hline NBR & 33.10750 & 131.05333 & 450 \\
\hline SUN & 32.87000 & 131.08933 & 1240 \\
\hline TAT & 33.01317 & 130.89900 & 300 \\
\hline
\end{tabular}

Table 6.4: Summary of station specifications at Aso caldera

ray path and source effects (see section 8.2.1). In addition to shear-wave splitting parameters we calculated the ratio between the $P$ - and the $S$-velocities.

\subsubsection{GPS data}

Crustal deformation data can be obtained from the Geospatial Information Authority of Japan (GSI, Miyazaki et al., 1996, 1997, 1998; references from Sagiya et al., $2000)^{20}$. We downloaded coordinates from 2001-2007 for 39 stations (see figure 6.5 and table 6.5) on Kyushu island around Aso volcano. Although many of the stations are not in the direct vicinity of the volcano, their data can be used to improve control over the actual area of interest when it comes to modelling of strain at a later point, as boundary effects or less well constrained strain estimates are moved further away from the caldera.

Processing Bad measurements had a marker in the coordinate files and were removed. The data were then filtered with a lowpass filter at a cutoff frequency of $1 / 15$ days $^{-1}$, the same as the displacement data from lake Taupo. Occasional data gaps were filled by linear interpolation. Error bars are not provided with the data, but we estimate standard deviations of the raw data (with a linear trend removed) of approximately $0.003 \mathrm{~m}, 0.003 \mathrm{~m}$ and $0.009 \mathrm{~m}$ on the east, north and vertical component, respectively. The ratio of standard deviation on the vertical component $\left(\sigma_{V}\right)$ to either one of the horizontal components $\left(\sigma_{H}\right)$ of $\frac{\sigma_{V}}{\sigma_{H}}=3$ is in agreement with findings from other studies of the Japanese GPS network (Liu et al., 2010).

High seasonal variations as seen in figure 6.7 have to be considered in order to avoid misinterpreting them as volcanic signal. Dong et al. (2002) find that $66 \%$ of the observed power of seasonal deformation in GPS time series can be attributed to pole tides ${ }^{21}$ and seasonal mass redistribution. Furthermore they emphasise contributions from atmospheric changes as well as thermal expansion of bedrock. Sudo and Hurst (1998) find high seasonal temperature variations associated with the volcanic

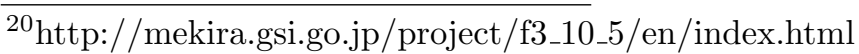

${ }^{21}$ i.e. variations due to the Chandler wobble 


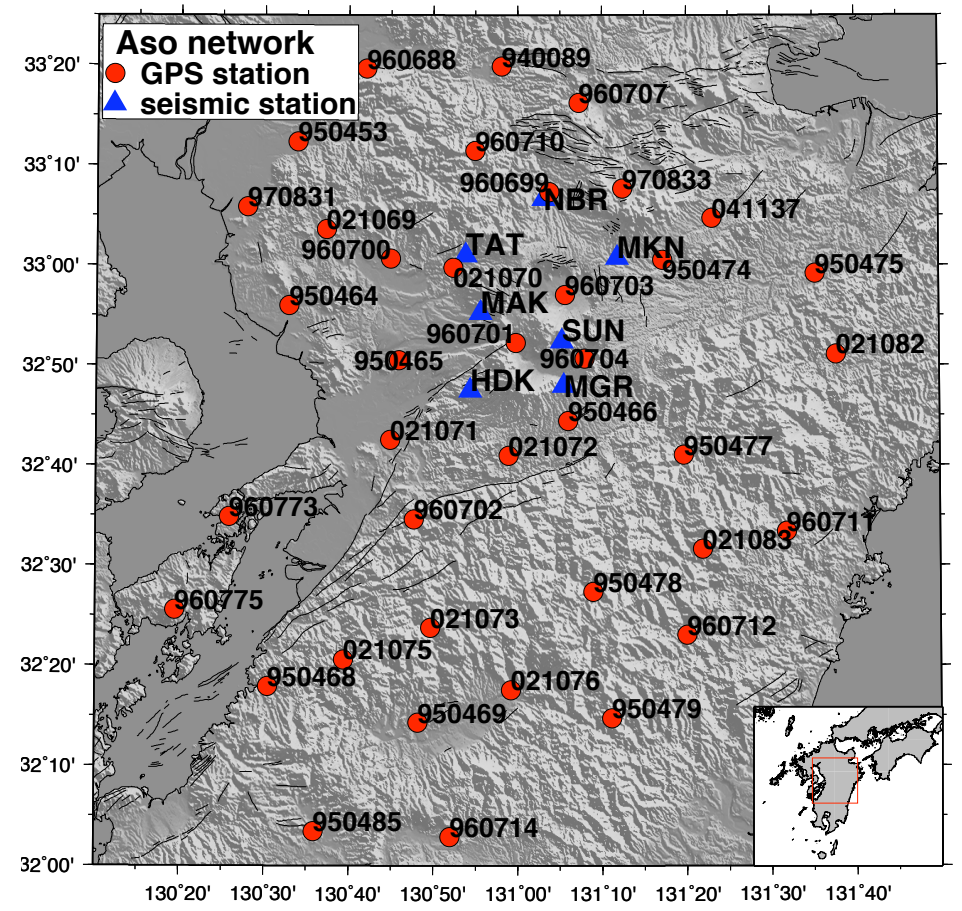

Figure 6.5: Station network around Aso caldera, Japan

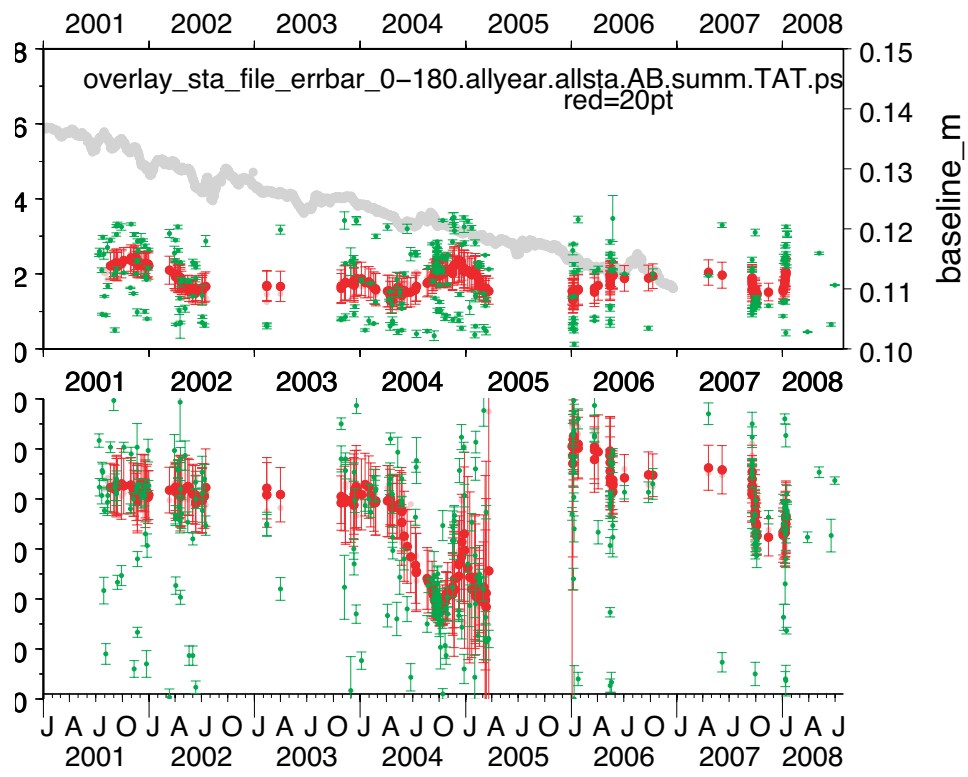

Figure 6.6: Moving means over $\phi$ and $\delta t$ for station TAT. Distinct change in 2004-2005. Red dots are means over 20 points, and green dots are individual results, respectively. Error bars are $95 \%$ confidence intervals. Grey timeseries in upper plot is GPS baseline length over a station pair crossing TAT. Figure from M. Savage (2010, pers.comm.). 


\begin{tabular}{|l||l|l|l|}
\hline station & latitude $\left(^{\circ}\right)$ & longitude $\left(^{\circ}\right)$ & altitude $(\mathrm{m})$ \\
\hline \hline 021069 & 33.0601 & 130.6218 & 72.8922 \\
\hline 021070 & 32.9959 & 130.8727 & 290.1468 \\
\hline 021071 & 32.7092 & 130.7484 & 69.5622 \\
\hline 021072 & 32.6829 & 130.9820 & 484.9498 \\
\hline 021073 & 32.3961 & 130.8273 & 343.8611 \\
\hline 021075 & 32.3435 & 130.6565 & 380.4383 \\
\hline 021076 & 32.2924 & 130.9864 & 223.0167 \\
\hline 021082 & 32.8522 & 131.6292 & 214.5306 \\
\hline 021083 & 32.5279 & 131.3647 & 867.9792 \\
\hline 041137 & 33.0788 & 131.3840 & 519.2998 \\
\hline 940089 & 33.3311 & 130.9678 & 149.6305 \\
\hline 950453 & 33.2059 & 130.5646 & 69.7936 \\
\hline 950464 & 32.9335 & 130.5477 & 51.4785 \\
\hline 950465 & 32.8421 & 130.7648 & 92.2459 \\
\hline 950466 & 32.7407 & 131.0993 & 725.2995 \\
\hline 950468 & 32.2992 & 130.5068 & 42.8648 \\
\hline 950469 & 32.2387 & 130.8026 & 193.5713 \\
\hline 950474 & 33.0090 & 131.2861 & 579.8798 \\
\hline 950475 & 32.9869 & 131.5878 & 200.5367 \\
\hline 950477 & 32.6848 & 131.3276 & 346.5336 \\
\hline 950478 & 32.4566 & 131.1489 & 617.9128 \\
\hline 950479 & 32.2459 & 131.1852 & 943.0970 \\
\hline 950485 & 32.0575 & 130.5978 & 215.7243 \\
\hline 960688 & 33.3272 & 130.7012 & 85.7572 \\
\hline 960699 & 33.1220 & 131.0629 & 469.7276 \\
\hline 960700 & 33.0113 & 130.7486 & 115.3843 \\
\hline 960701 & 32.8707 & 130.9962 & 450.7903 \\
\hline 960702 & 32.5767 & 130.7949 & 287.7844 \\
\hline 960703 & 32.9507 & 131.0934 & 544.6408 \\
\hline 960704 & 32.8450 & 131.1321 & 650.9308 \\
\hline 960707 & 33.2703 & 131.1211 & 381.1693 \\
\hline 960710 & 33.1906 & 130.9157 & 877.8208 \\
\hline 960711 & 32.5570 & 131.5307 & 124.6132 \\
\hline 960712 & 32.3844 & 131.3340 & 285.2834 \\
\hline 960714 & 32.0477 & 130.8656 & 287.3429 \\
\hline 960773 & 32.5817 & 130.4304 & 50.8671 \\
\hline 960775 & 32.4263 & 130.3236 & 621.4484 \\
\hline 970831 & 33.0974 & 130.4650 & 50.2677 \\
\hline 970833 & 33.1278 & 131.2068 & 976.2254 \\
\hline & & & \\
\hline
\end{tabular}

Table 6.5: Summary of GPS station specifications at Aso caldera 
hydrothermal system that could contribute to bedrock volume changes. The large scale of the hydrothermal system at Aso volcano (Hase et al., 2005), the vicinity to other volcanoes and their hydrothermal systems on Kyushu and the location of the caldera at latitudes where snow loading changes significantly from winter to summer explain the high seasonal variations observed in the GPS data. By simply applying a highpass filter we could not suppress these variations. However, we mostly calculate strain for time spans on the order of years (period I, II, III and VI, see 6.6), where seasonal variations should have little effect. Period IV and V cover time spans likely to experience seasonal effects and should hence be interpreted carefully. We keep this limitation in mind. Also note that different stations can have varying amounts of seasonal variations e.g. due to differences in precipitation.
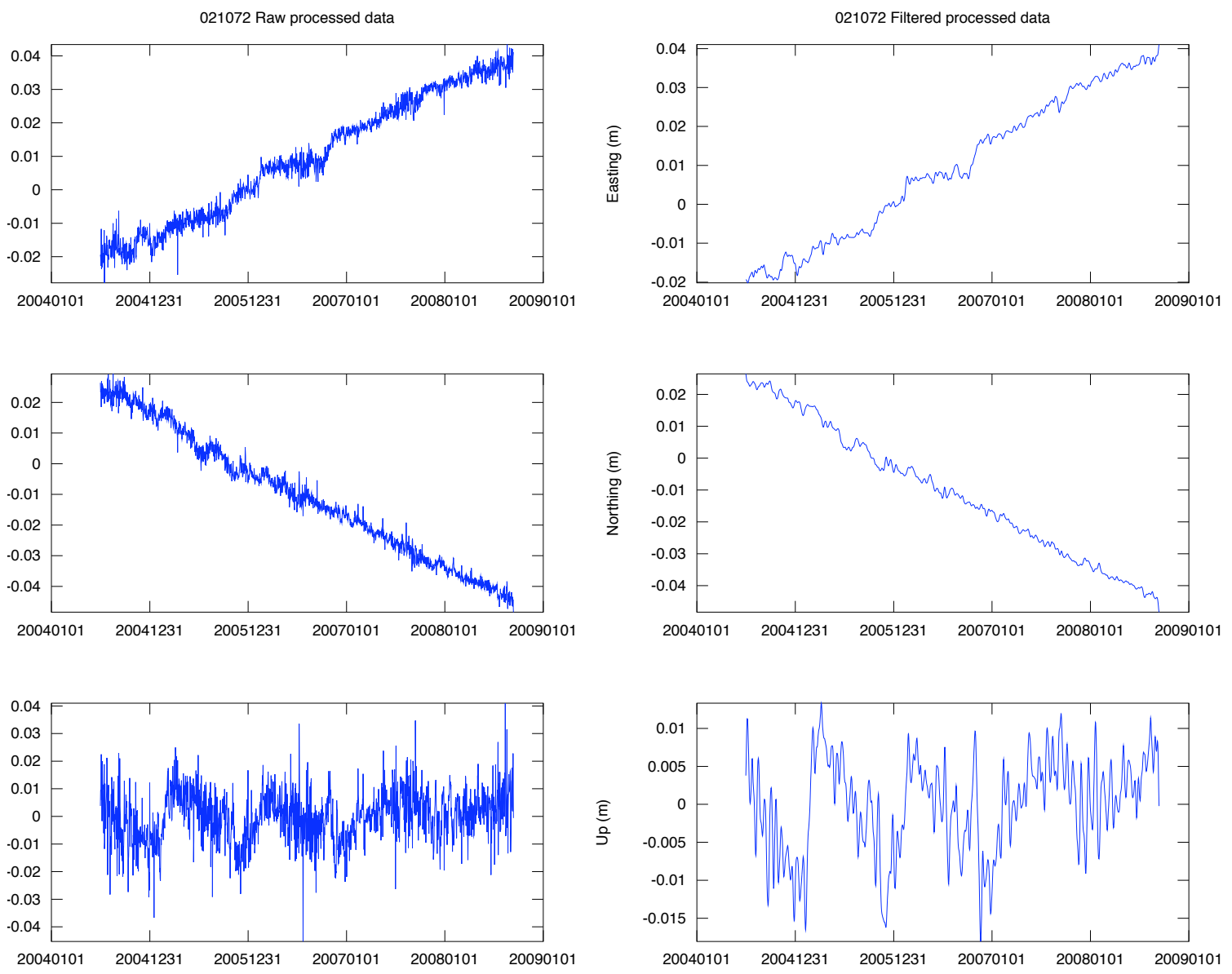

Figure 6.7: GPS timeseries for station 021072 at Aso caldera. Left side are raw data with outliers removed, right side show timeseries filtered with a $1 / 15$ days $^{-1}$ lowpass. Note the annual cycle especially on the vertical component (seasonal variation!). 
Definition of deformation episodes After preliminary inspection of strain and shear-wave splitting in the area, we defined six periods in which we grouped the data, as can be seen in table 6.6. The strain calculations as well as shear-wave splitting results that have been used for this classification will be discussed in sections 7 and 8.

\begin{tabular}{|l||l|l|l|}
\hline Period & time span & shear-wave splitting & 2D strain \\
\hline \hline I & 2001-2002 & stable & $\begin{array}{l}\text { stable, compression } \\
\text { dominating in caldera }\end{array}$ \\
\hline II & 2003 & stable & $\begin{array}{l}\text { change to extension in } \\
\text { caldera }\end{array}$ \\
\hline III & Jan-Oct 2004 & $\begin{array}{l}\text { beginning change in } \\
\text { fast direction }\end{array}$ & $\begin{array}{l}\text { compression dominat- } \\
\text { ing in caldera }\end{array}$ \\
\hline IV & Nov 2004- Jan 2005 & peak in fast direction & $\begin{array}{l}\text { possibly near-surface } \\
\text { extension in caldera }\end{array}$ \\
\hline V & Feb-Mar 2005 & $\begin{array}{l}\text { fast direction chang- } \\
\text { ing back to initial } \\
\text { state }\end{array}$ & $\begin{array}{l}\text { no areal strain in } \\
\text { caldera }\end{array}$ \\
\hline VI & 2006-2007 & stable & $\begin{array}{l}\text { stable, compression } \\
\text { dominating in caldera }\end{array}$ \\
\hline
\end{tabular}

Table 6.6: Definition of episodes for Aso volcano, based on shear-wave splitting (see figure 7.11 and appendix D) and strain analyses (see figure 7.14). For actual results and discussion of these analyses see sections 7 and 8 .

Parameters for strain calculation Parameters for strain calculation around Aso volcano are summarised in table 6.7. Similar to Taupo caldera, noise on the vertical component was high: As mentioned above, we do not get error bars with the data, but find an estimate of the typical standard deviations on the vertical of approximately three times the standard deviations on the horizontal components for stations within the Japanese network. We hence only look at 2D strain.

\begin{tabular}{|l|l|}
\hline Grid-cell size & $7 \mathrm{~km}$ \\
\hline No. of nearest neighbours & 4 \\
\hline Maximum station distance & $28 \mathrm{~km}$ \\
\hline
\end{tabular}

Table 6.7: Parameters for SSPX strain calculation for Aso volcano 


\subsection{Gisborne}

\subsubsection{Seismic data}

Two datasets were downloaded and processed for the Gisborne area around three distinct slow slip events. 1842 earthquakes with hypocentres between latitudes $-37.8^{\circ}$ and $-39.3^{\circ}$ and longitudes $177.4^{\circ}$ and $178.6^{\circ}$ and magnitudes higher than 2.0 recorded on two stations (KNZ and PUZ, see table 6.8) between 01/01/2002 - 30/06/2005 cover two slow slip events, around October 2002 and November 2004, respectively. The second dataset uses the same boundaries and magnitude limits, however for a different time span: For a slow slip event in March/April 20102492 earthquakes from 01/01/2009 - 17/08/2010 recorded on three stations (KNZ, MWZ and PUZ) were analysed. The station locations together with the location of the GPS stations (see table 6.9) in the area can be seen in figure 6.8.

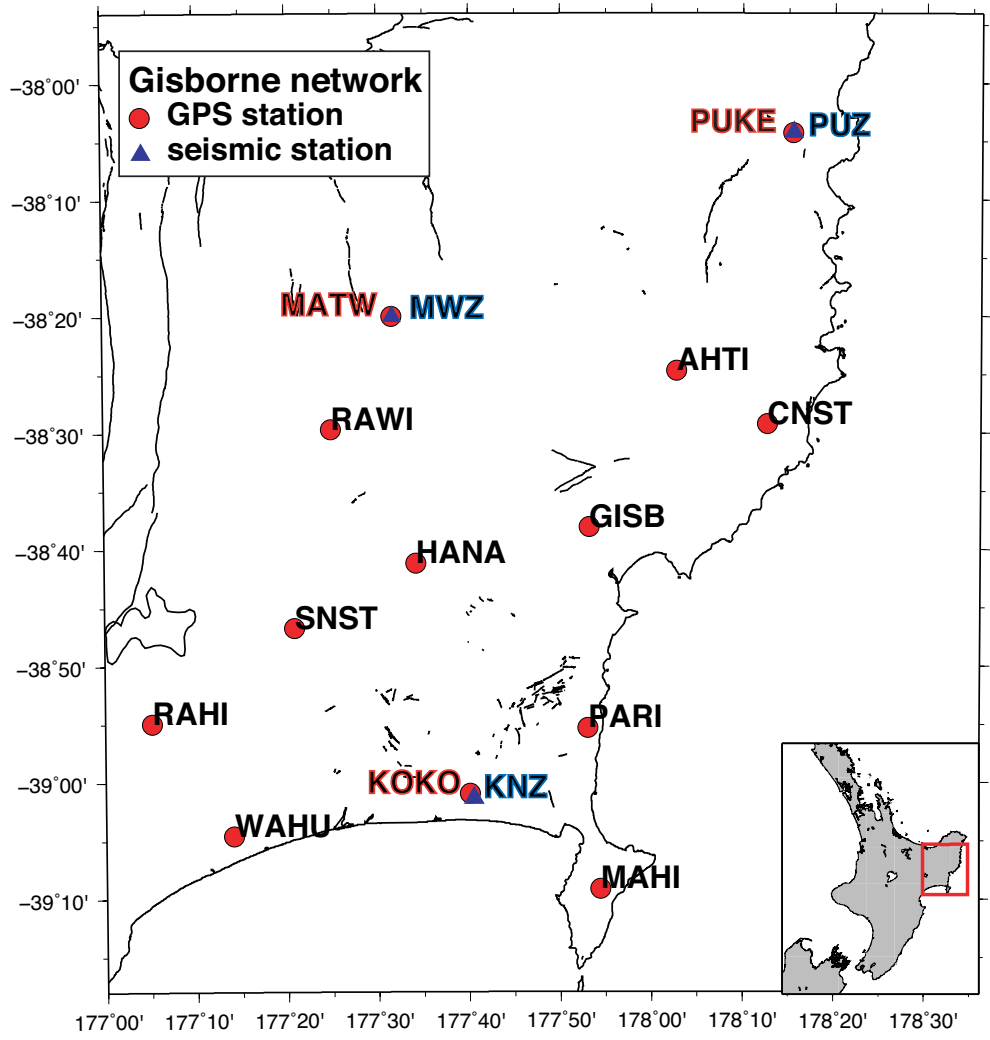

Figure 6.8: Station network in the area of the Gisborne slow slip events, New Zealand. Red dots are GPS stations, blue triangles are seismometers. At places where GPS stations and seismometers are located close together the names are coloured red and blue, respectively, for better distinction. Black lines on onshore part are active faults. Refer to table 6.9 for timing of GPS installations. 


\begin{tabular}{|c|c|c|c|c|}
\hline 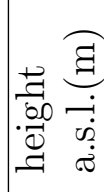 & $\approx 2$ & $\approx$ & 苫 & $\stackrel{8}{\dddot{P}}$ \\
\hline $\begin{array}{l}0 \\
0 \\
0 \\
\underline{0} \\
.0 \\
.0 \\
00 \\
0 \\
0\end{array}$ & 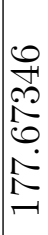 & 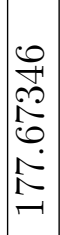 & 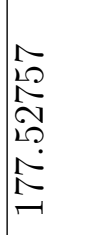 & $\begin{array}{l}\Omega \\
D \\
0 \\
L \\
\sim \\
\sim \\
\infty \\
1 \\
-1\end{array}$ \\
\hline 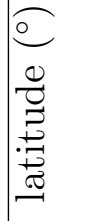 & 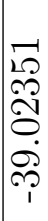 & 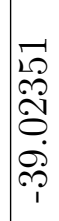 & 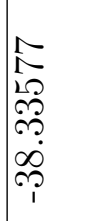 & 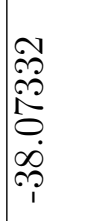 \\
\hline 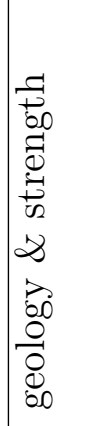 & 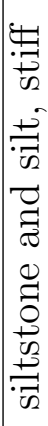 & 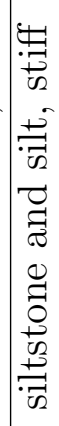 & 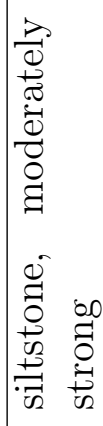 & 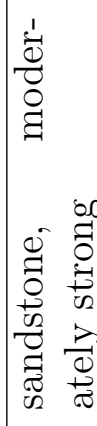 \\
\hline 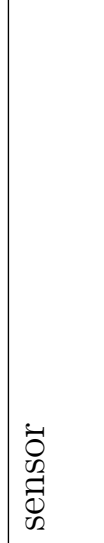 & 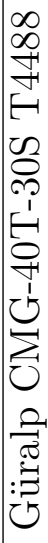 & 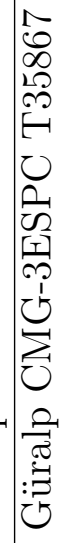 & 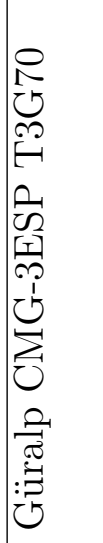 & 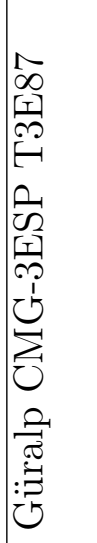 \\
\hline 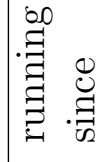 & 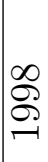 & 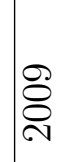 & 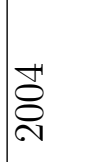 & 离 \\
\hline 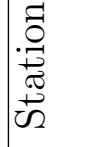 & $=$ & 齐 & 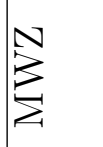 & 食 \\
\hline
\end{tabular}

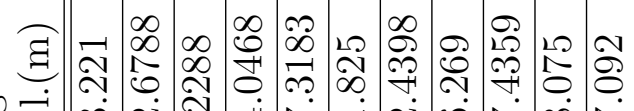

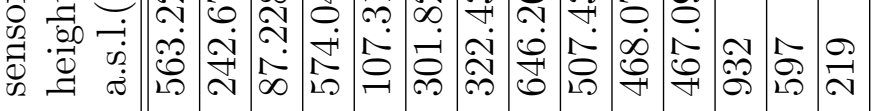
N

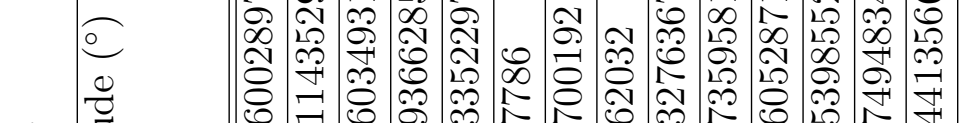
๑ँ

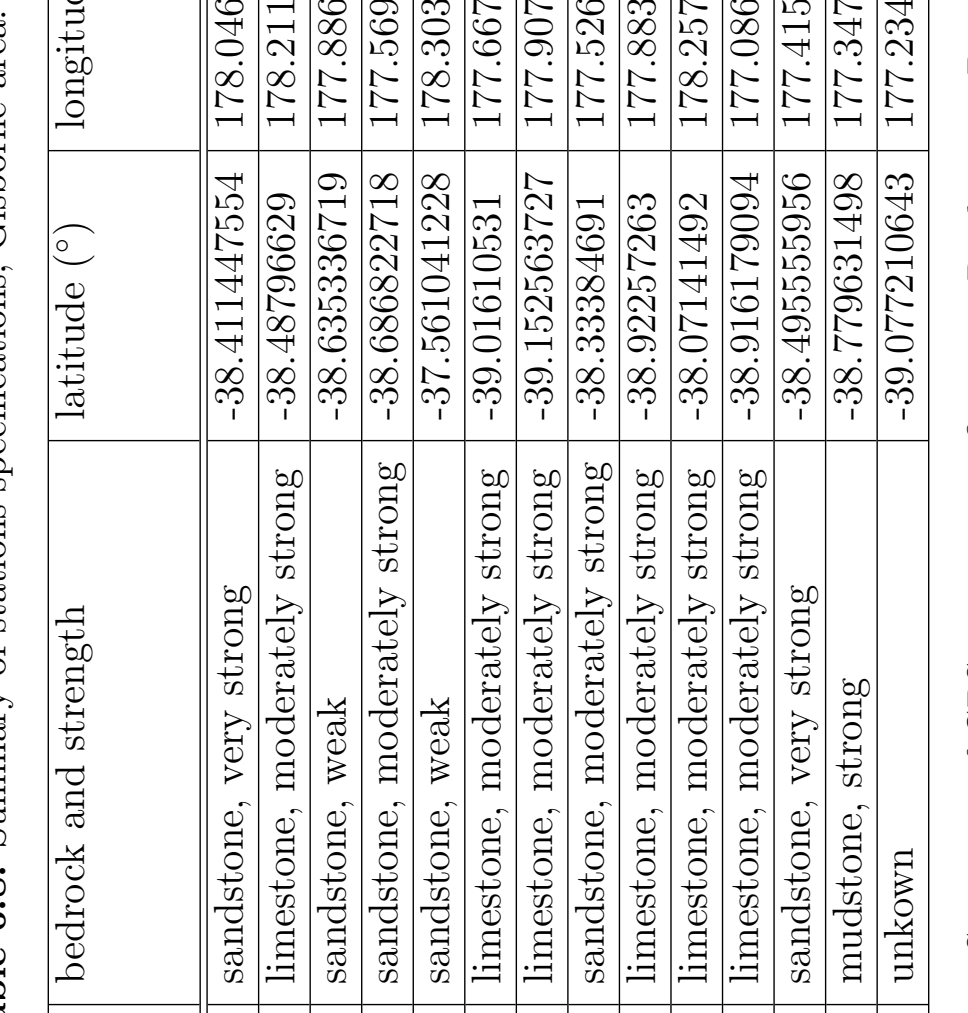
ह $\stackrel{\infty}{\Xi}$ 寻

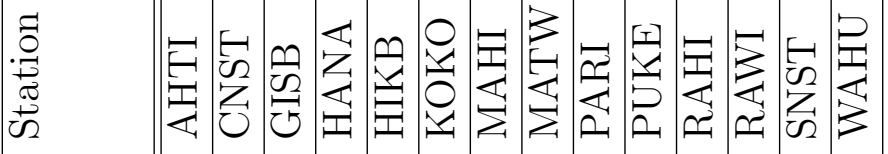




\subsubsection{GPS data}

For the Gisborne slow slip events, we used the GPS timeseries from the GeoNet webpage $^{22}$ for the station GISB (figure 6.9). Several slow slip events can be seen in the timeseries. We chose three of them, the ones with the largest displacement magnitudes, to compare them to shear-wave splitting results.

In addition, we downloaded processed position timeseries from the same webpage to calculate the strain field.

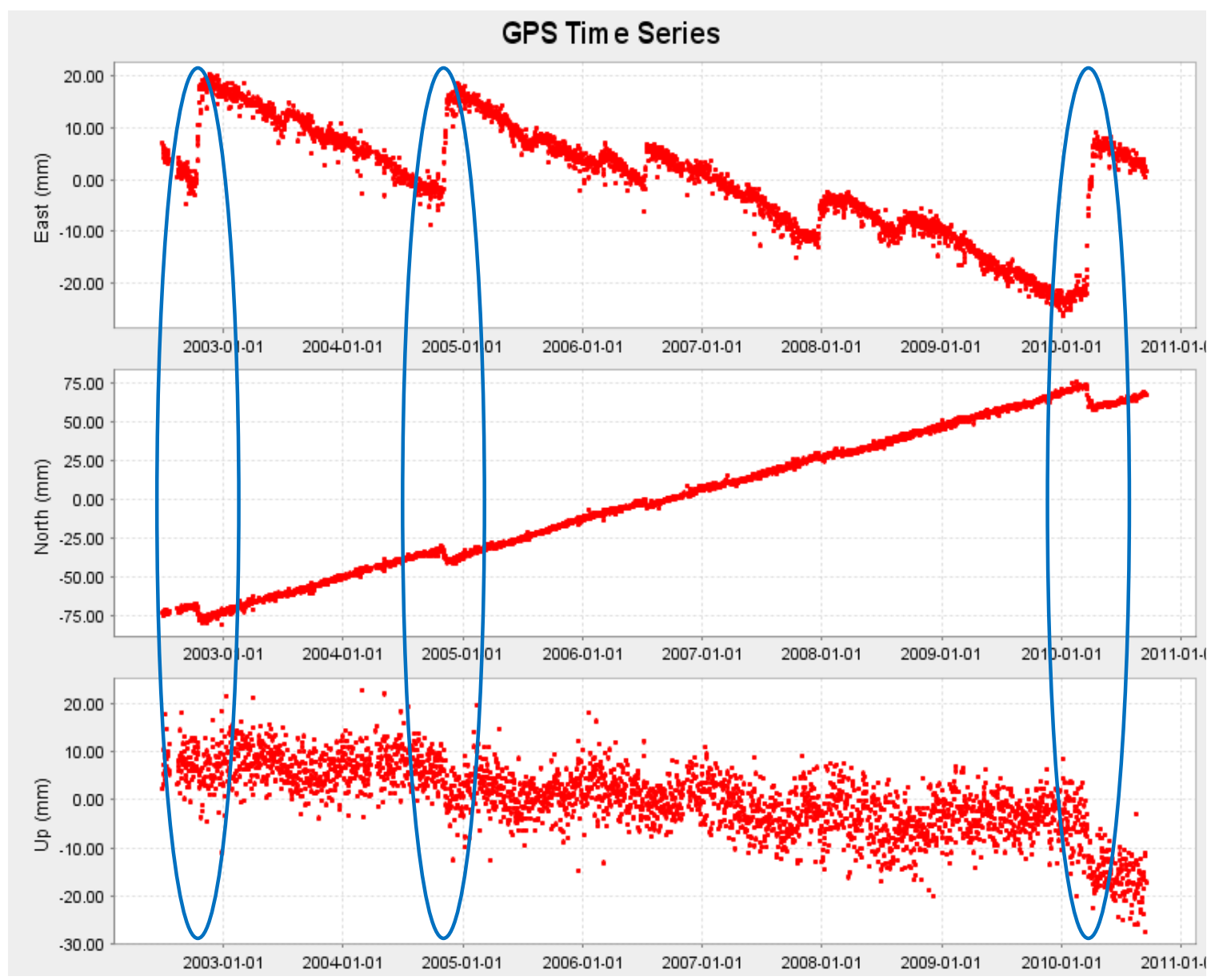

Figure 6.9: GPS timeseries for station GISB showing slow slip events. Red dots are individual cGPS readings regionally filtered. Blue circles indicate the time of the three slow slip events chosen for this study. Figure modified from http://www.geonet.org.nz/resources/gps/timeseries/index.html.

Processing The data obtained from GeoNet has station positions in millimetres relative to NZGD2000 position and a time stamp in seconds since January 01, 1970,

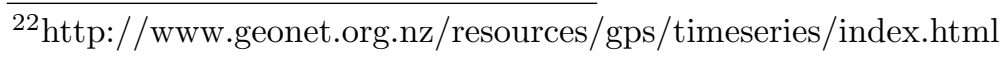




\begin{tabular}{|l|l|}
\hline Grid-cell size & $10 \mathrm{~km}$ \\
\hline No. of nearest neighbours & 4 \\
\hline Maximum station distance & $40 \mathrm{~km}$ \\
\hline
\end{tabular}

Table 6.10: Parameters for SSPX strain calculation for Raukumara Peninsula

00:00 GMT. An automated GeoNet procedure has already removed obvious outliers and the data are "regionally filtered", i.e. corrections have been made for errors more or less common to all stations in New Zealand, such as erroneous satellite orbits or large-scale mass loading. We then filtered the data with a $1 / 15$ days $^{-1}$ lowpass filter and filled data gaps with linear interpolation. Standard deviations from the raw $^{23}$ time series for station GISB are $0.004 \mathrm{~m}, 0.003 \mathrm{~m}$, and $0.010 \mathrm{~m}$ on the east, north and vertical components, respectively, and on the same order for all other stations.

Definition of deformation episodes For the Gisborne slow slip events, we only analysed the strain field for the last event (March 2010), due to better station coverage during that time. Deformation is divided into three periods: before, during and after the slow slip event, respectively. The corresponding dates are 06/08/2009 - 01/01/2010, 01/01/2010 - 01/05/2010 and 01/05/2010 - 01/09/2010.

Parameters for strain calculation Parameters for strain calculation on the Raukumara peninsula are summarised in table 6.10. As for the other two areas we only look at $2 \mathrm{D}$ strain.

${ }^{23}$ i.e. before the regional filtering by GeoNet and hence probably maximum estimates 


\section{Results}

\subsection{Taupo caldera}

\subsubsection{Shear-wave splitting}

All in all 856 earthquakes yielded shear-wave splitting results with grades A or B. Their depths are up to $244 \mathrm{~km}$, with magnitudes $\mathrm{M}_{L}$ between 2.0 and 5.6 (see table 7.1). Figure 7.1 shows the subset of all used events on the left, compared to the whole earthquake catalog for that time and region on the right. The subset is a good representation of the whole catalog. Table 7.2 summarises the number of events that yielded splitting measurements at each station for the different time spans and the number of catalogue vs. manual picks. This emphasises the importance of manual picking: $49.8 \%$ of all shear-wave splitting results in this area are calculated from manual picks. $\sim 39 \%$ of all results come from earthquakes deeper than $15 \mathrm{~km}$, i.e. from below the bottom of the crust in the TVZ (Stern, 1985). Those events are separated from the analyses to illuminate crustal processes ${ }^{24}$.

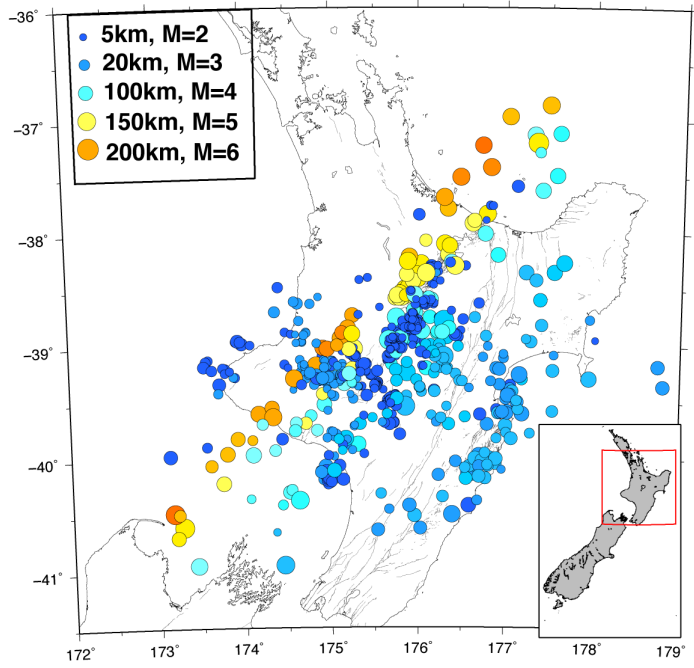

(a) Events with A or B grade splitting measurements

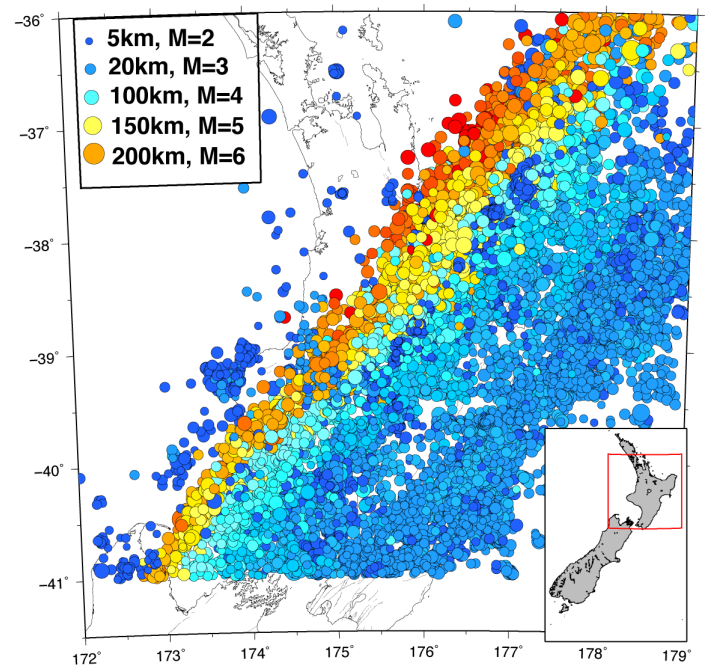

(b) All earthquakes downloaded from the GeoNet catalog

Figure 7.1: Used earthquakes vs. all downloaded events for lake Taupo. Both subsets delineate the subducting Pacific plate as well as shallow crustal earthquakes in the TVZ and around Mt. Taranaki.

Averaging techniques Plots of the individual results at any one station show large scatter (grey dots in figure 7.2). To make possible patterns more clearly visible,

\footnotetext{
${ }^{24}$ Note that we might still get some contribution from ray paths sampling the mantle when separating the evens according to their hypocentre depth. We recommend for example ray tracing to make a separation of only those events that sampled the crust for future studies of this area.
} 


\begin{tabular}{|l||l|l||l||l|l|}
\hline & minimum & maximum & & minimum & maximum \\
\hline \hline latitude $\left(^{\circ}\right)$ & -40.94 & -36.86 & longitude $\left(^{\circ}\right)$ & 173.14 & 177.96 \\
\hline distance $(\mathrm{km})$ & 0.256 & 316.07 & depth $(\mathrm{km})$ & 0.41 & 244.19 \\
\hline magnitude & 2.0 & 5.6 & $\begin{array}{l}\text { angle of inci- } \\
\text { dence }\left(^{\circ}\right)\end{array}$ & 0.9 & 27.5 \\
\hline$\delta \mathrm{t}(\mathrm{s})$ & 0 & 1.0 & $\begin{array}{l}\text { standard devia- } \\
\text { tion of } \delta \mathrm{t}(\mathrm{s})\end{array}$ & $<0.002$ & 0.25 \\
\hline$\phi\left(^{\circ}\right)$ & -89 & 90 & $\begin{array}{l}\text { standard devia- } \\
\text { tion of } \phi\left(^{\circ}\right)\end{array}$ & 0.25 & 25 \\
\hline
\end{tabular}

Table 7.1: Summary of parameters from shear-wave splitting at Taupo caldera

\begin{tabular}{|l||l|l|l|l|l|l|l|l||l|}
\hline Station & period I & II & III & IV & V & VI & VII & total \\
\hline \hline ARAZ & catalogue & 0 & 0 & 13 & 8 & 3 & 24 & 18 & 66 \\
\hline & manual & 0 & 0 & 33 & 11 & 2 & 31 & 17 & 94 \\
\hline \hline HATZ & catalogue & 14 & 34 & 125 & 14 & 29 & 67 & 34 & 317 \\
\hline & manual & 21 & 38 & 84 & 34 & 55 & 100 & 18 & 350 \\
\hline \hline KATZ & catalogue & 0 & 59 & 134 & 6 & 7 & 10 & 3 & 219 \\
\hline & manual & 16 & 12 & 72 & 10 & 32 & 44 & 4 & 190 \\
\hline \hline POIZ & catalogue & 5 & 6 & 16 & 3 & 2 & 15 & 13 & 60 \\
\hline & manual & 0 & 0 & 5 & 3 & 0 & 47 & 8 & 63 \\
\hline \hline RATZ & catalogue & 24 & 7 & 52 & 0 & 4 & 37 & 49 & 173 \\
\hline & manual & 7 & 15 & 41 & 12 & 27 & 33 & 6 & 141 \\
\hline \hline RITZ & catalogue & 11 & 15 & 47 & 4 & 11 & 24 & 22 & 134 \\
\hline & manual & 6 & 11 & 17 & 4 & 24 & 51 & 18 & 131 \\
\hline \hline WATZ & catalogue & 12 & 22 & 88 & 6 & 7 & 33 & 25 & 193 \\
\hline & manual & 15 & 13 & 62 & 31 & 33 & 55 & 12 & 221 \\
\hline \hline WHTZ & catalogue & 1 & 1 & 12 & 0 & 3 & 29 & 27 & 73 \\
\hline & manual & 0 & 0 & 6 & 3 & 4 & 16 & 8 & 37 \\
\hline
\end{tabular}

Table 7.2: Number of splitting measurements, Taupo caldera 
previous studies such as Savage et al. (2010b) have created averages from a larger amount of measurements. Here, an arithmetic mean over a 30 point moving window was applied to smooth the scattered data. This technique reduces the influence of outliers and can show trends of the whole dataset. However, it has to be stressed that the method can also create misleading results at stations with only few data overall or at certain times. In addition, actual abrupt changes in the results (for instance a step from one fast direction to another) will be masked by a slope as the moving window gradually averages over the change. The standard error is calculated and can indicate the amount of scatter within each window. It should be noted that this error results only from the variation between the measurements in each window, not from the actual measurement error of each result. Also note that the error bars of subsequent average points are not independent, in fact for a 30 point moving average only the errors of every 30th point will be independent (E. Smith, pers.comm., 2011).

Another way of obtaining averages for a fixed time is to stack the error contours (after Wolfe and Silver, 1998) of the individual splitting measurements for that period of time. This method will not be bound to the individual result from each measurement, but will also take into account other combinations of $\phi$ and $\delta t$ with a low second eigenvalue (and hence a certain likelihood to be the real splitting parameters, see section 5.1). However, testing of this method showed large deviations from the individual results and the moving means. We have not fully developed this method and hence will not rely on these averages or include them in any interpretation. Further testing and development will be necessary to investigate the effects of this type of statistics.

The moving means at the different stations show complicated changes with no obvious pattern. Particularly the fast directions exhibit large variations on different time scales in a seemingly chaotic manner (see figure 7.3). The 30 point mean $\delta t$ is roughly between $\sim 0.2-0.7 \mathrm{~s}$. While some stations have relatively stable $\delta t$ over time, station HATZ for example shows almost cyclic changes (figure 7.2). Comparing the moving means at the different stations is difficult: $\phi$ is too chaotic to be related. $\delta t$ shows one signal common to the three stations HATZ, RATZ and WATZ that appears for all measurements and for the restricted crustal subset: A relative high in $\delta t$ towards the end of the investigated time period (figure 7.3). Each station, however, also has individual highs in delay time at different times unrelated to other stations, which makes it difficult to interpret this change.

Mode When analysing fast directions and delay times, the mean as discussed above can be one way of illuminating trends in the measurements. Another way is to look at the $\phi$ and $\delta t$ that occur most often, i.e. the mode of the distribution. If $\delta t$ and $\phi$ are normally distributed, this will be the arithmetic mean as calculated above. If the two parameters show deviations from Gaussian distribution, however, the mode will not be the same as the arithmetic mean. Figure 7.4 illustrates the difference.

As we plot the fast directions as circular histograms on each station in map view, we are practically looking at modes. Hence, it is appropriate to calculate modes 

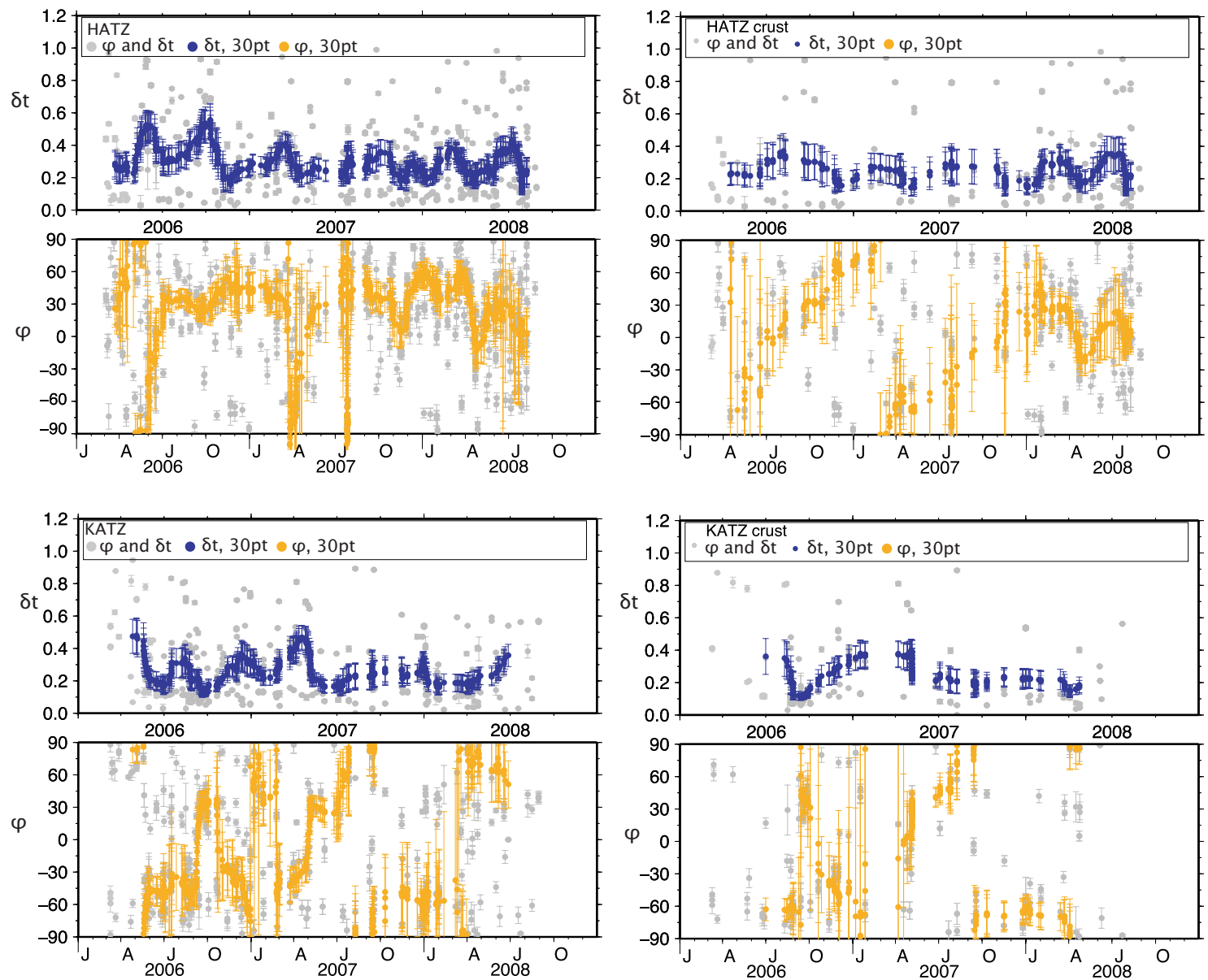

Figure 7.2: 30 point moving means for $\phi$ (orange) and $\delta t$ (blue) at stations HATZ (upper half) and KATZ (lower half). Left plots are all measurements, right hand side only for events from less than $15 \mathrm{~km}$ depth (referred to as "crust" for this region). Error bars are $95 \%$ confidence intervals. Note that the error bars on the moving means are strongly correlated, since only every 30 th point is independent. Grey dots are individual measurements. 

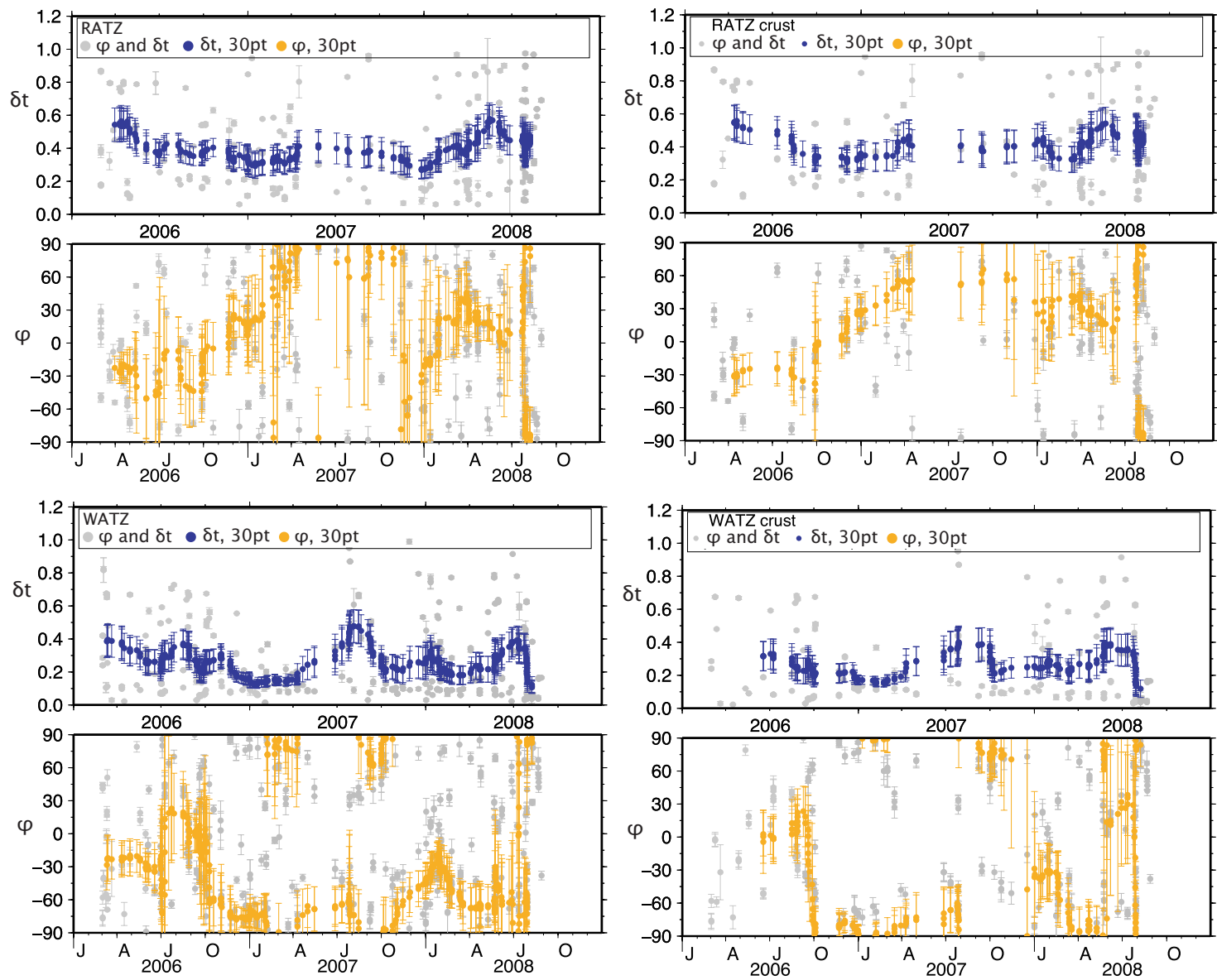

Figure 7.3: 30 point moving means for $\phi$ (orange) and $\delta t$ (blue) at stations RATZ (upper half) and WATZ (lower half). Left plots are all measurements, right hand side only for crustal events. Error bars are $95 \%$ confidence intervals. Note that the error bars on the moving means are strongly correlated, since only every 30th point is independent. Grey dots are individual measurements. Note that the moving means in $\phi$ for WATZ are cut due to the range of directions in the plot and the circular nature of the data. Chosing a range of 60 to 240 would solve this problem, but make it more difficult to compare to the other stations. 


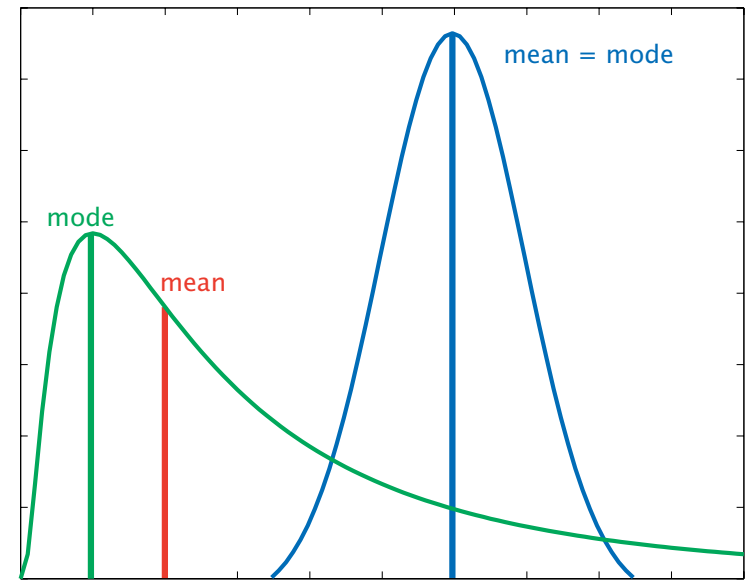

Figure 7.4: Illustration of the difference between mean and mode for non-Gaussian distribution: The blue curve is a Gaussian distribution, where the mean and the mode are the same (blue bar). The green curve is a non-Gaussian distribution, where the mean (red bar) and the mode (green bar) are not the same. Curves and bar positions are schematic only.

and plot them over time instead of averages. While this is straightforward for delay time, the fast directions create a problem due to their circular nature, as regular mode calculations will only "see" numbers from 0 to 180, instead of directions where 0 and 180 are the same. As the scatter of fast directions is high and only yields an accuracy of approximately $10^{\circ}$ or even less, we avoided the circular nature of the data by introducing $10^{\circ}$ intervals. Instead of allowing all possible fast directions, we fixed $\phi$ to the centre of the $10^{\circ}$ interval it is lying in. If $0^{\circ} \leq \phi<10^{\circ}$ it will be fixed to $5^{\circ}$, if $10^{\circ} \leq \phi<20^{\circ}$ it wil be fixed to $15^{\circ}$ and so on. With this modification, the mode can be easily calculated.

Plots of the moving mode over time, however, do not show any coherent patterns (figure 7.5). Scatter is high even for long window lengths and there appears to be a step-like variation. This is most likely due to the creation of bins for fast directions (see exaplanation above) in combination with sets of individual results somehow dominating the mode of a number of windows, until their effect dies off and another set of individual measurements dominates the next few windows. We conclude that with the calculation at this point we cannot discern systematic changes in shear-wave splitting parameters, if they exist for the investigated stations. It is possible that further development of the method could reveal more systematic changes. In addition, examining changes of the median over time could be a useful proxy for changes in fast direction or delay time. There is also the issue of the "circular nature" of fast directions, and the resulting non-Gaussian distribution. It has been suggested to average xy-components or tangent of fast direction instead (G. Bokelmann, pers.comm., 2011). A new method would have to be developed and tested. Due to the time constraints of this study we skip this step, but highly recommend it for future analyses of shear-wave splitting.

Spatial variations The high variability of fast direction discussed above will have an influence on plots of the fast directions for the different periods: Most stations do not exhibit one stable $\phi$ for any given deformation episode. Hence, looking at circular histograms ("rose diagrams") for each period will show a set of direction for most 


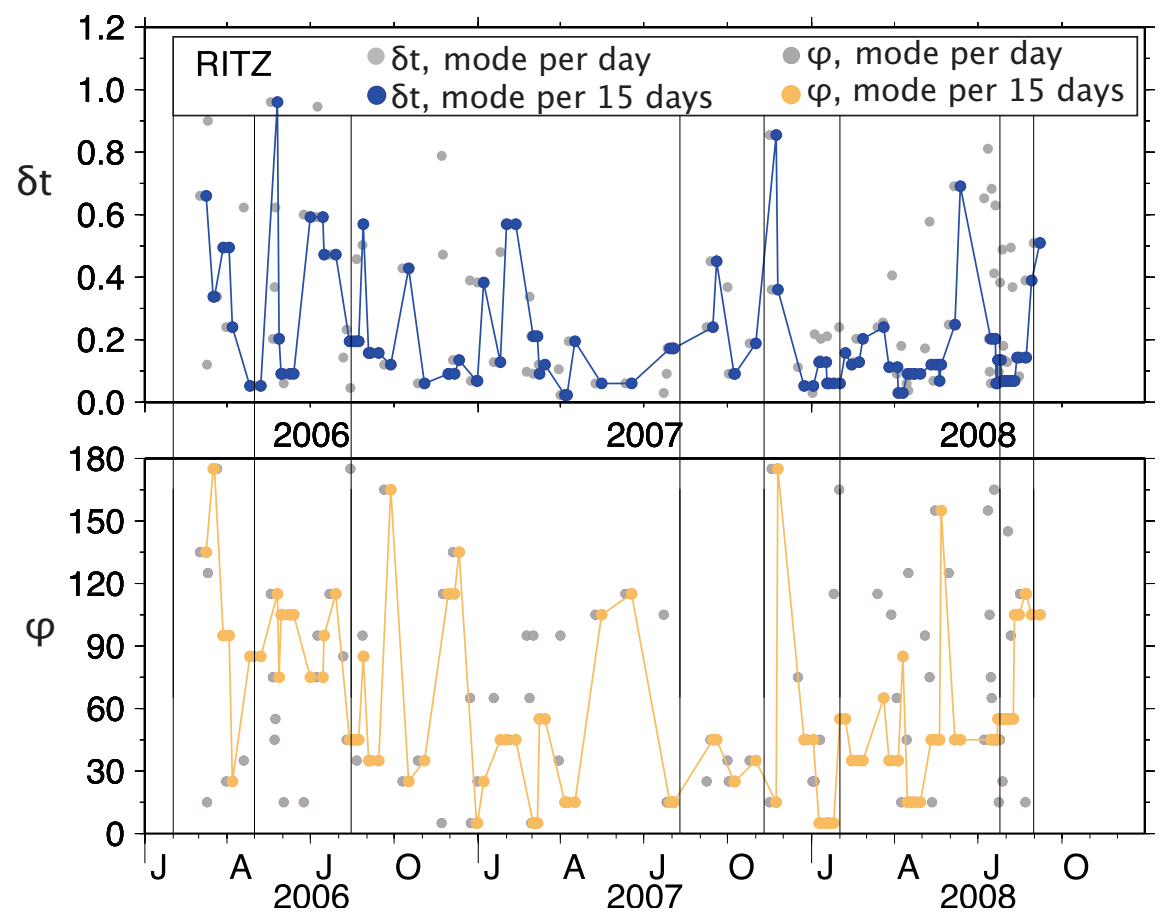

Figure 7.5: Mode for moving 15 day windows at station RITZ, sliding in one day increments. Grey dots are modes per day, and blue and orange dots are modes per 15 days for $\delta t$ and $\phi$, respectively. The vertical black lines separate the different periods.

times and stations. Figure 7.6 shows rose diagrams for the whole time and events with hypocentres shallower than $15 \mathrm{~km}$ (shallow) compared to hypocentres deeper than $15 \mathrm{~km}$ (deep). In general the difference between fast directions for deep and shallow earthquakes is small. We find a $\mathrm{N}$ to $\mathrm{NE}$ trend at most stations, sometimes in combination with a second peak of $\phi$ trending NW. Table 7.3 summarises the mean fast direction at each station for shallow and deep events.

\subsubsection{Deformation: time series and strain}

After the processing described in section 6.1.2, the deformation data were analysed more thoroughly. To investigate temporal variations and compare them to shearwave splitting results, we chose three different ways of looking at deformation.

Time series Most of the GPS sensors were not available for the entire time, but were installed some time between 2006 and 2007. Comparisons to shear-wave splitting were hence only possible for a limited period of time, depending on the station. We plotted the displacement relative to the first day of the timeseries (filtered with a lowpass of $1 / 15$ days $^{-1}$ as described in section 6.1.2) to compare it to temporal variations in shear-wave splitting at sites where a GPS sensor and a seismometer were relatively close together. Although all components are considered, the vertical 

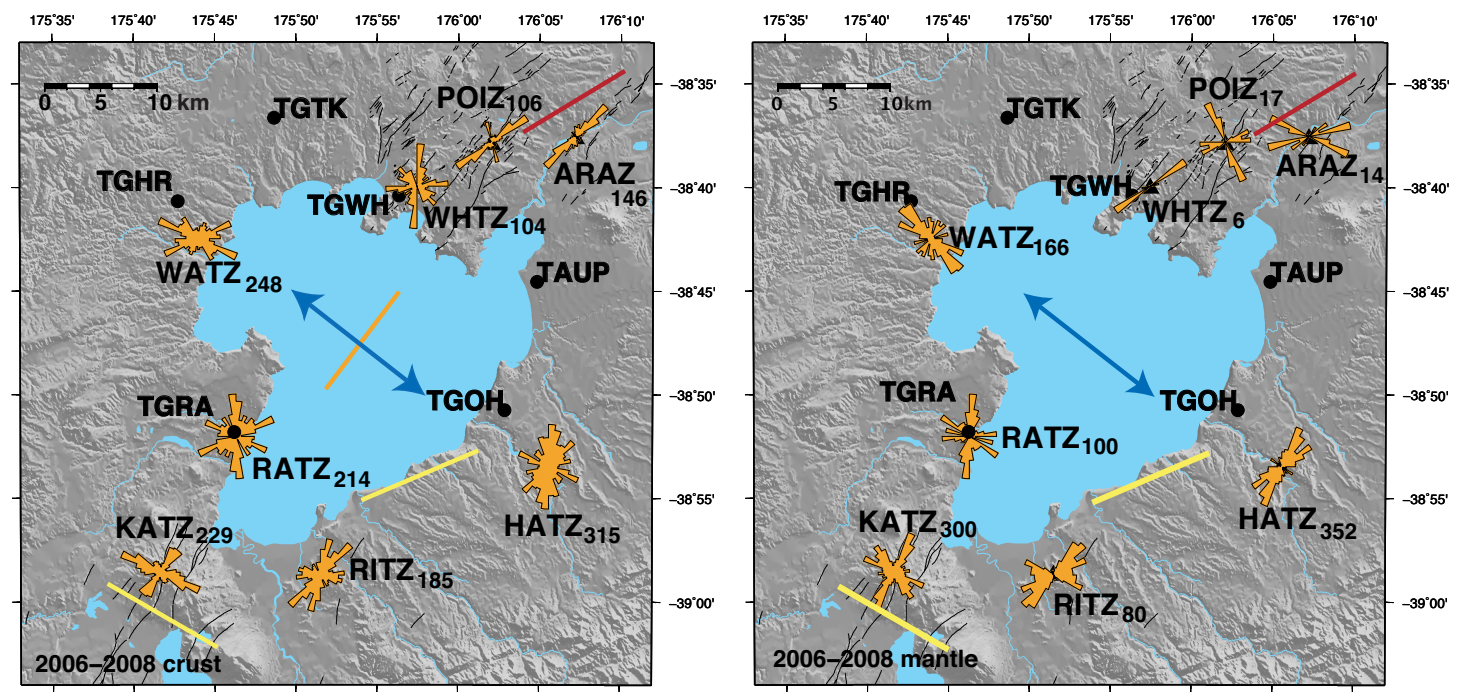

(a) Rose diagrams for 2006-2008 for shallow (b) Rose diagrams for 2006-2008 for deep events events

Figure 7.6: Rose diagrams for crust and mantle events for Taupo caldera 2006-2008. Orange rose diagrams show fast directions at different stations, black dots and associated names are GPS stations. Three distant GPS stations are outside of this zoom. The subscript numbers next to seismometer names are the number of $\mathrm{A}$ and $\mathrm{B}$ grade measurements at this station for the respective subset of events. The blue arrow indicates extension at $128^{\circ} \pm 11^{\circ}$ (Darby et al., 2000). The yellow bars show $S_{H, \max }$ estimates of $120^{\circ} \pm 12^{\circ}$ (left) and $67^{\circ} \pm 11^{\circ}$ (right) after Sherburn et al. (2009) and the red bar is $S_{H, \max }=58^{\circ}$ after Hurst et al. (2002). The orange bar in the centre of the lake for the lefthand figure represents the mean $\phi$ for stations with low scatter. See section 8.1.1 for further discussion.

\begin{tabular}{|l||l|l|l||l|l|l|}
\hline station & $\begin{array}{l}\text { no. } \\
\text { shallow } \\
\text { meas. }\end{array}$ & $\phi\left(^{\circ}\right)$ & $\delta t(\mathrm{~s})$ & $\begin{array}{l}\text { no. } \\
\text { deep } \\
\text { meas. }\end{array}$ & $\phi\left(^{\circ}\right)$ & $\delta t(\mathrm{~s})$ \\
\hline \hline ARAZ & 146 & $39 \pm 2$ & $0.23 \pm 0.01$ & $14^{*}$ & $86 \pm 8$ & $0.35 \pm 0.04$ \\
\hline HATZ & 315 & $10 \pm 6$ & $0.24 \pm 0.01$ & 352 & $39 \pm 2$ & $0.34 \pm 0.01$ \\
\hline KATZ & $229^{*}$ & $-82 \pm 10$ & $0.25 \pm 0.01$ & $300^{*}$ & $-23 \pm 15$ & $0.28 \pm 0.01$ \\
\hline POIZ & 106 & $42 \pm 5$ & $0.33 \pm 0.02$ & $17^{*}$ & $-73 \pm 62$ & $0.68 \pm 0.06$ \\
\hline RATZ & $214^{*}$ & $22 \pm 16$ & $0.43 \pm 0.02$ & $100^{*}$ & $-21 \pm 12$ & $0.36 \pm 0.02$ \\
\hline RITZ & 185 & $39 \pm 7$ & $0.26 \pm 0.02$ & 80 & $66 \pm 5$ & $0.38 \pm 0.03$ \\
\hline WATZ & $248^{*}$ & $-81 \pm 7$ & $0.26 \pm 0.01$ & 166 & $-38 \pm 5$ & $0.26 \pm 0.01$ \\
\hline WHTZ & $104^{*}$ & $-36 \pm 132$ & $0.38 \pm 0.02$ & 7 & $57 \pm 3$ & $0.54 \pm 0.09$ \\
\hline
\end{tabular}

Table 7.3: Summary of fast directions and delay times with standard errors for shallow and deep events at lake Taupo. A* denotes high scatter or bimodal distribution of $\phi$ for that subset and station and thus meaningless mean value. 
component has a relatively low signal-to-noise ratio and hence temporal variations might not be resolved accurately, as discussed in section 6.1.2. The time series by themselves show individual station displacement on the order of $\mathrm{mm} / \mathrm{yr}$. Stations to the west and the east of the lake show similar signals (figure 7.1.2 and 7.6). A signal of rapid deformation which is particularly strong on the east component of stations TGOH, TGWH and TAUP (east and north of the lake) and which has been identified previously by Jolly et al. (2008) does not appear as clearly after our processing, but is still visible.

Figure 7.7: (Figure on next page) Filtered GPS timeseries for Taupo caldera. Red dots separate different periods, with period number in red on the east component. Note the different time scales for stations RGAR and TGWH. Stations on the left hand side are roughly west of the lake, stations on the right roughly east and station TGWH is in the centre north of the lake, see also figure 7.6. Also note the different vertical scales for different stations, misleadingly making for example the "step" in 2007 appear larger on the western than on the eastern stations.

Baseline changes Following the approach from Savage et al. (2010b), we calculated baseline lengths and their variations in time to compare them to shear-wave splitting. Error bars depend on the error from the individual station positions: Assuming a mean standard deviation of $0.002 \mathrm{~m}$ on both east and north components gives an approximate mean standard deviation of $\sim 0.0028 \mathrm{~m}$ for each component of the $2 \mathrm{D}$ baseline, and hence an overall estimate of a baseline standard deviation of $0.004 \mathrm{~m}$.

The baselines standing alone have two main features, if we only consider variations above the noise level. A small subset shows either relatively stable length or an almost constant decrease in distance between two given stations (see figure 7.8b). Baselines in the other subset exhibit an increase (decrease in one case) in length of approximately $5 \mathrm{~mm}$ in 2007 and sometimes a weaker but still anomalous signal in the second half of 2008 , as the example in figure $7.8 \mathrm{a}$ shows. The fact that the anomalies are slightly above statistical significance considering the estimated maxmimum error of $4 \mathrm{~mm}$ is supported by the fact that the change is visible on most stations at the same time. The overall changes in baselines are also statistically significant and the examples in figure 7.8 show NW-SE extension and NE-SW contraction, respectively.

2D Strain For period I with only two GPS sensors it is not possible to calculate strain. Although periods II and III have three and four sensors, respectively, the resolution is not good enough to detect local variations of the principal strain direc- 

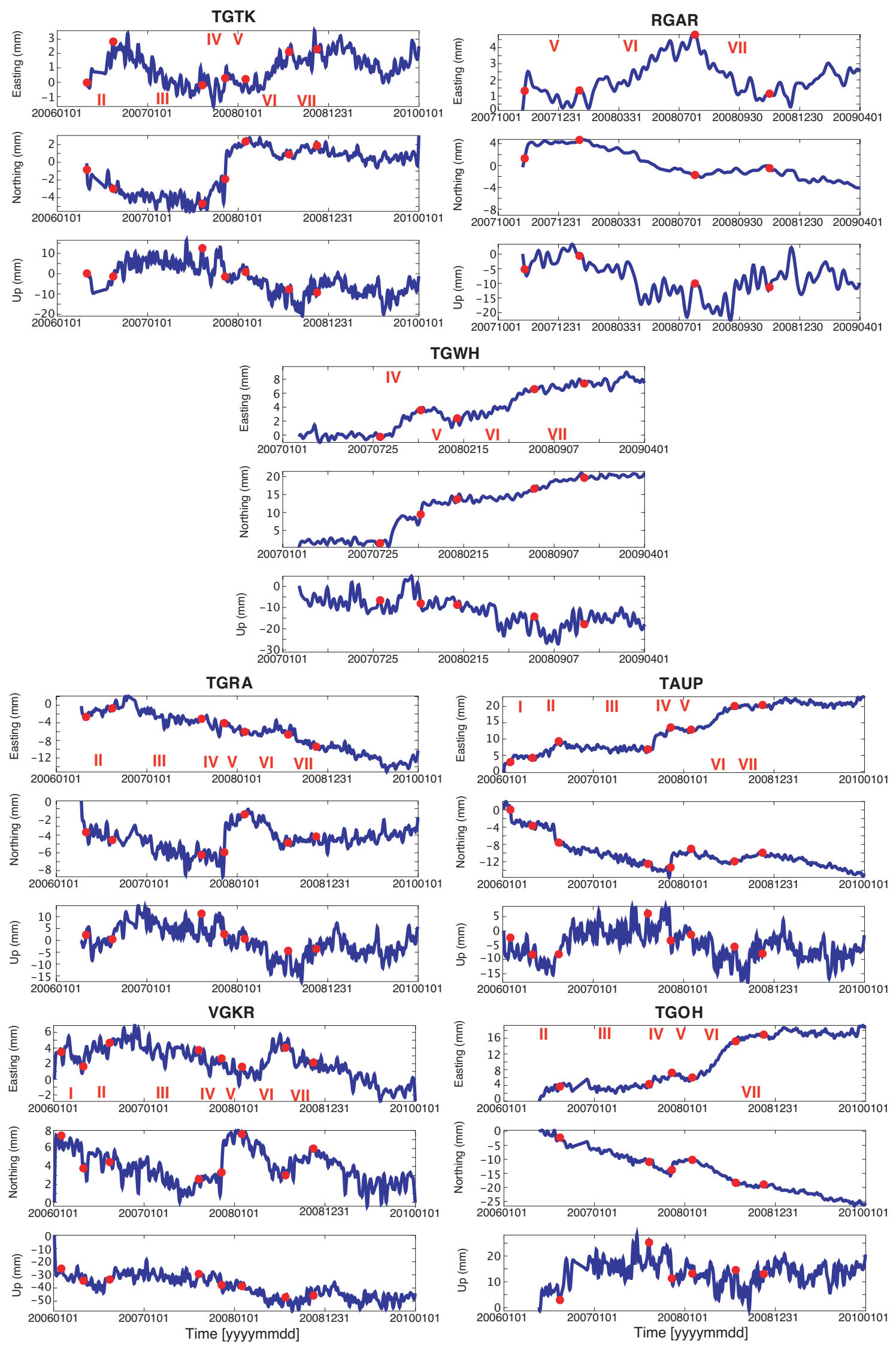

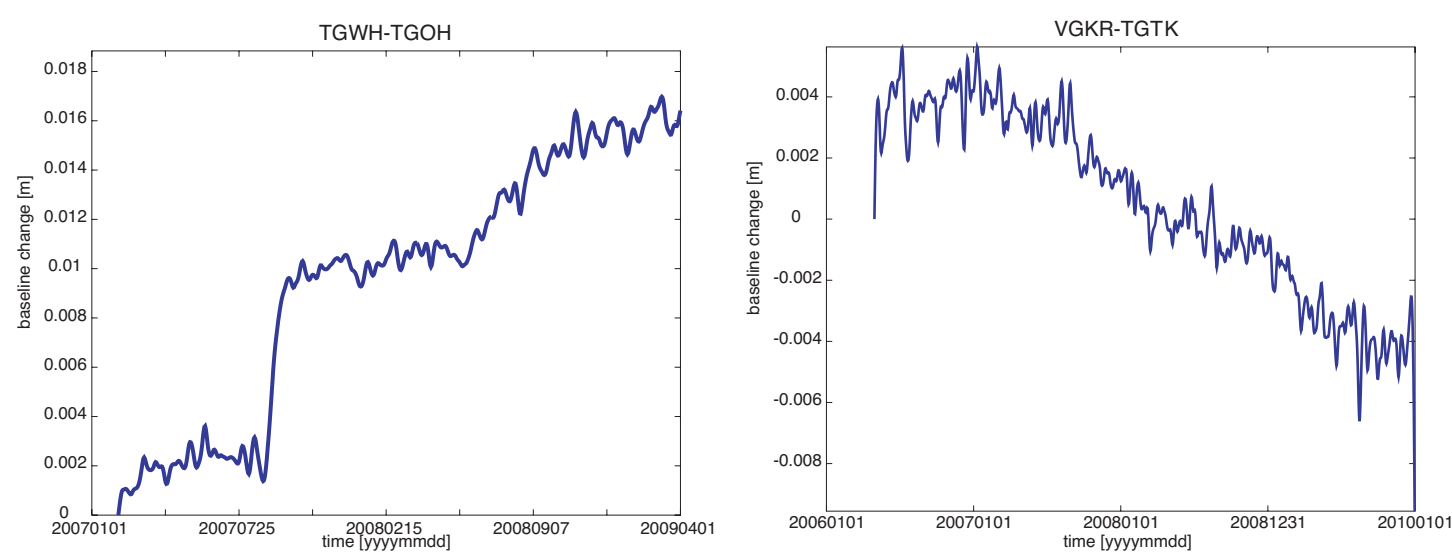

(a) Baseline change between stations TGWH and $\mathrm{TGOH}$

(b) Baseline change between stations VGKR and TGTK

Figure 7.8: Two examples for baseline changes around lake Taupo. Data are filtered with $1 / 15$ days $^{-1}$. Refer to figure 6.1 for station locations. Note the different time spans covered by the station pairs.

tions. Both show extensional areal strain of up to $\sim 3 * 10^{-7} \pm 3 * 10^{-7}$ dominating $^{25}$ with shortening axes trending roughly $\mathrm{NE}^{26}$. Periods IV to VII deserve more attention as they show higher local variations due to better station coverage (see figure 7.9). The northeast corner of the investigated area stands out with compressional areal strain of up to $\sim 6 * 10^{-8} \pm 2 * 10^{-7}$ for periods V through VII compared to the overall extensional areal dilatation of up to $\sim 8 * 10^{-7} \pm 4 * 10^{-7}$ for the rest of the region. The high error estimates, however, make this compressional trend statistically insignificant (see appendix A for errors in each grid cell). Although shortening axes still show a general NE trend, there are significant deviations from this trend in individual grid cells. While the southern end shows shortening axes oriented NNE, the central parts occasionally have almost perpendicular orientations and a trend towards NW can be observed for the northwestern shore of the lake. Looking at strain directions at the actual stations quantifies those trends: While at stations TAUP, TGRA, TGTK and TGWH the maximum change in orientation of strain shortening axes is roughly $45^{\circ}$, the direction undergoes a change of almost $90^{\circ}$ at station TGOH between periods IV and V (table 7.5). Figure 7.9 shows the strain fields for the four better constrained periods; the directions for the different grid cells and periods are summarised in table 7.4 .

${ }^{25}$ Note that errors on the GPS time series were retrieved at a later stage. Rough sensitivity test show that the mean standard deviations of $0.002 \mathrm{~m}, 0.002 \mathrm{~m}$ and $0.009 \mathrm{~m}$ for $\mathrm{E}, \mathrm{N}$, and Z, respectively, approximately 100-fold the error from the inversion. Any error estimates on areal dilatation from lake Taupo will be based on this approximation. Appendix A shows the error in $2 \mathrm{D}$ strain values due to the inversion.

${ }^{26} \mathrm{SSPX}$ does not provide error bars on directions in the individual grid cells. Since we chose an inversion method so that the results would be relatively stable in this configuration, we estimate that the mean error on directions will not exceed a few degrees. 


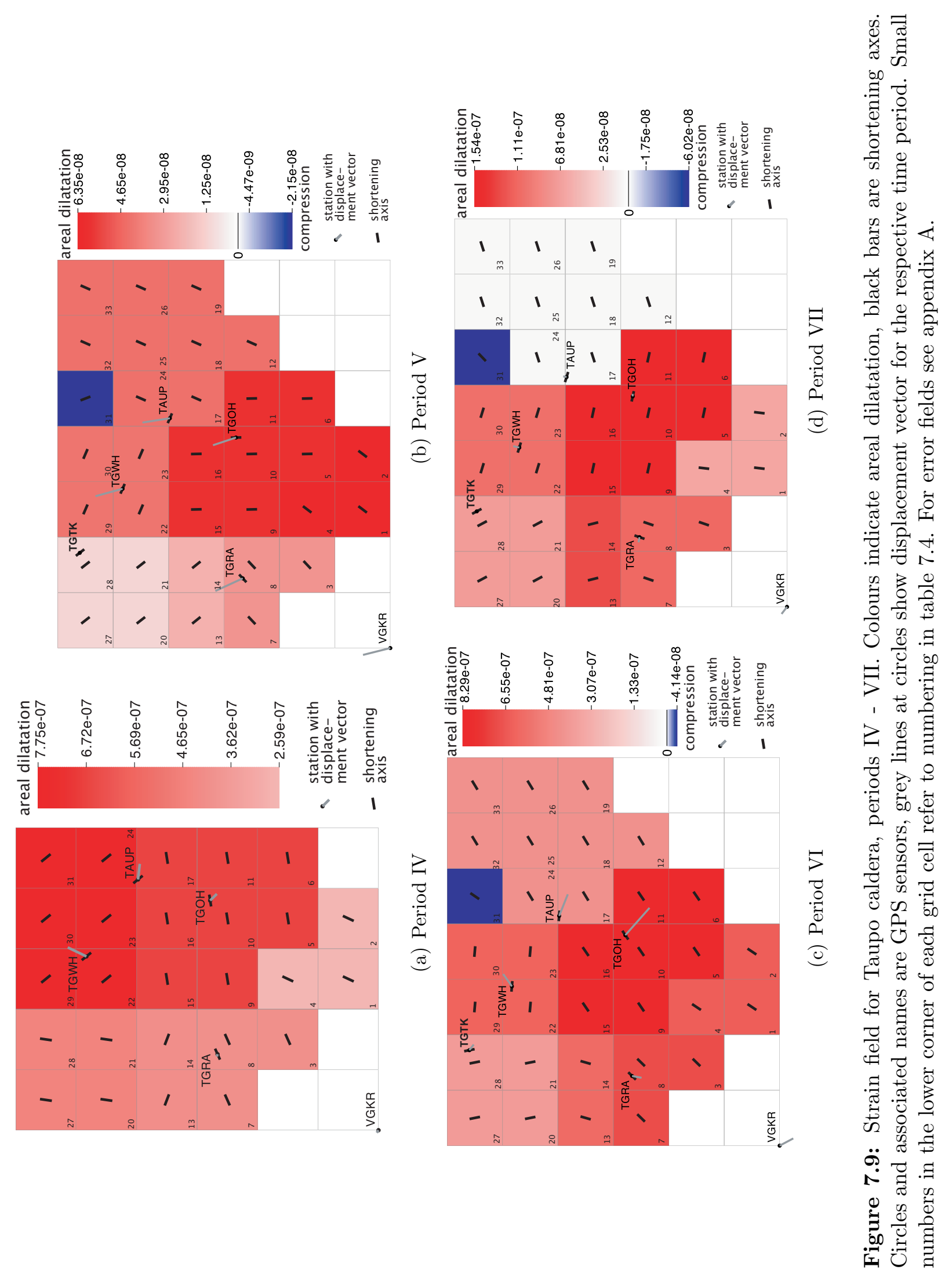




\subsection{Aso caldera}

\subsubsection{Shear-wave splitting}

Out of 1272 events recorded on the whole network, 933 earthquakes recorded on seven stations yielded 3746 splitting measurements with an A or B grade quality. The variation in parameters of these events is summarised in table 7.6 and their locations ${ }^{27}$ can be seen in figure 7.10 .

To avoid the interpretation of changes in shear-wave splitting parameters due to source effects, we defined seven clusters in the vicinity of Aso caldera. Results from this subset of splitting measurements will be discussed in section 8.2.1.

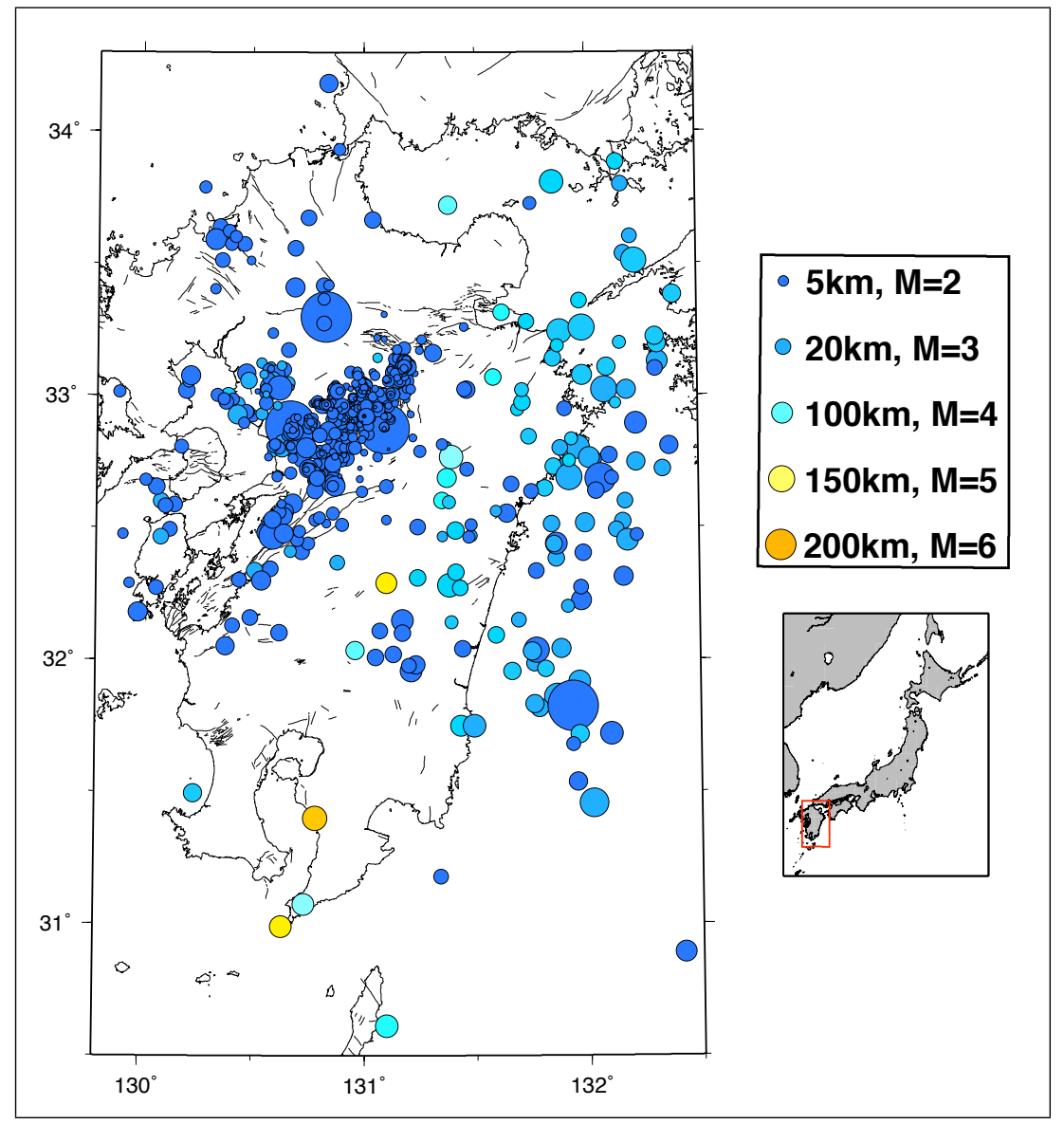

Figure 7.10: Earthquakes that yielded $\mathrm{A}$ and $\mathrm{B}$ grade splitting measurements at Aso caldera.

\footnotetext{
${ }^{27}$ Events were located only based upon stations in the local network around Aso caldera. Thus, locations outside of the network have high uncertainties associated with them. Further work for publication of this project will only use events from within the network.
} 


\begin{tabular}{|l||l|l|l|l||l||l|l|l|l|}
\hline cell & IV & V & VI & VII & cell & IV & V & VI & VII \\
\hline \hline 1 & 204.90 & 217.09 & 213.69 & 186.72 & 18 & - & 203.69 & 238.71 & 252.14 \\
\hline 2 & 204.90 & 217.09 & 213.69 & 186.72 & 19 & - & 203.69 & 238.71 & 252.14 \\
\hline 3 & 245.87 & 226.71 & 224.26 & 198.87 & 20 & 188.24 & 142.02 & 168.78 & 151.96 \\
\hline 4 & 204.90 & 217.09 & 213.69 & 186.72 & 21 & 188.24 & 142.02 & 168.78 & 151.96 \\
\hline 5 & 260.90 & 178.73 & 235.61 & 102.18 & 22 & 141.01 & 113.44 & 94.91 & 107.13 \\
\hline 6 & 260.90 & 178.73 & 235.61 & 102.18 & 23 & 141.01 & 113.44 & 94.91 & 107.13 \\
\hline 7 & 245.87 & 226.71 & 224.26 & 198.87 & 24 & 141.01 & 203.69 & 238.71 & 252.14 \\
\hline 8 & 245.87 & 226.71 & 224.26 & 198.87 & 25 & - & 203.69 & 238.71 & 252.14 \\
\hline 9 & 260.90 & 178.73 & 235.61 & 102.18 & 26 & - & 203.69 & 238.71 & 252.14 \\
\hline 10 & 260.90 & 178.73 & 235.61 & 102.18 & 27 & 142.02 & 188.24 & 168.78 & 151.96 \\
\hline 11 & 260.90 & 178.73 & 235.61 & 102.18 & 28 & 142.02 & 188.24 & 168.78 & 151.96 \\
\hline 12 & - & 203.69 & 238.71 & 252.14 & 29 & 141.01 & 113.44 & 94.91 & 107.13 \\
\hline 13 & 110.97 & 141.17 & 195.90 & 165.94 & 30 & 141.01 & 113.44 & 94.91 & 107.13 \\
\hline 14 & 110.97 & 141.17 & 195.90 & 165.94 & 31 & 141.01 & 158.88 & 214.41 & 226.17 \\
\hline 15 & 260.90 & 178.73 & 235.61 & 102.18 & 32 & - & 203.69 & 238.71 & 252.14 \\
\hline 16 & 260.90 & 178.73 & 235.61 & 102.18 & 33 & - & 203.69 & 238.71 & 252.14 \\
\hline 17 & 260.90 & 203.69 & 238.71 & 252.14 & & & & & \\
\hline
\end{tabular}

Table 7.4: Summary of directions for strain shortening axes at Taupo caldera for periods IV - VII. Cell numbers refer to numbers in figure 7.9. Note that those are the exact values calculated by SSPX and error bars are not provided. Errors are likely to be on the order of several degrees (see discussion of $2 \mathrm{D}$ strain field above).

\begin{tabular}{|l||l|l|l|l|}
\hline station & IV & V & VI & VII \\
\hline \hline TAUP & 141.01 & 113.44 & 94.91 & 107.13 \\
\hline TGOH & 260.90 & 178.73 & 235.61 & 102.18 \\
\hline TGRA & 245.87 & 226.71 & 224.26 & 198.87 \\
\hline TGTK & 188.24 & 142.02 & 168.78 & 151.96 \\
\hline TGWH & 141.01 & 113.44 & 94.91 & 107.13 \\
\hline
\end{tabular}

Table 7.5: Strain shortening directions at stations around Lake Taupo for periods IV VII. Note that those are the exact values calculated by SSPX. Errors are likely to be on the order of several degrees. 


\begin{tabular}{|l||l|l||l||l|l|}
\hline & minimum & maximum & & minimum & maximum \\
\hline \hline latitude $\left(^{\circ}\right)$ & 30.61 & 34.18 & longitude $\left(^{\circ}\right)$ & 129.90 & 132.66 \\
\hline distance $(\mathrm{km})$ & 0.45 & 277.95 & depth $(\mathrm{km})$ & -2.06 & 174.86 \\
\hline magnitude & 0.2 & 6.0 & $\begin{array}{l}\text { angle of inci- } \\
\text { dence }\left(^{\circ}\right)\end{array}$ & 1.6 & 34.9 \\
\hline$\delta t(\mathrm{~s})$ & 0.01 & 0.4 & $\begin{array}{l}\text { standard devia- } \\
\text { tion of } \delta t(\mathrm{~s})\end{array}$ & $<0.0006$ & 0.09 \\
\hline$\phi\left(^{\circ}\right)$ & -89 & 90 & $\begin{array}{l}\text { standard devia- } \\
\text { tion of } \phi\left(^{\circ}\right)\end{array}$ & 1.25 & 25.00 \\
\hline
\end{tabular}

Table 7.6: Summary of parameters from shear-wave splitting at Aso volcano. Depth is in relation to sea level.

Time variations To investigate the effect of different averaging techniques and stacking of error contours (see section 8.1.1) research is currently being undertaken in a different project (M. Savage, 2010, pers.comm). As attempts to improve averaging at Taupo caldera did not have any significant success, we do not apply any of those techniques to Aso volcano. Instead we use the already existing averaging technique and focus on the relation to strain and stress modelling.

Although in general scatter of individual measurements is high, applying a moving mean as discussed before reveals interesting trends at Aso caldera. In agreement with findings by Savage et al. (2010b), fast directions are relatively stable throughout 2001 - 2007, except for a strong change in 2004. The change is particularly strong at station TAT (figure 7.11) and is accompanied by a subtle cycle of high-low-high delay times. The same $\delta t$ variation can observed at stations HDK, MAK and NBR to the west and the north of the caldera (see appendix D). Delay times had been fixed to a maximum of $0.4 \mathrm{~s}$ in Savage et al. (2008) and for consistency we took the same approach here. All stations show mean delay times of approximately $0.2 \mathrm{~s}$ and variations are on the order of $0.05-0.1 \mathrm{~s}$.

Spatial variations Only a small portion of events that yielded splitting measurements have hypocentres deeper than the estimated crustal thickness of $35 \mathrm{~km}$. To see long-term trends usually a high number of measurements is necessary, and thus scatter in $\phi$ from the few mantle events is high (figure 7.12). Yet, at stations MAK and HDK, for example, alignment of fast directions seems to be similar to the trends observed for crustal earthquakes. Shallow events $(<35 \mathrm{~km})$, however, exhibit clear trends. West of the caldera there is a dominant WNW strike of fast directions. In addition, stations TAT and HDK show a secondary peak in fast directions oriented ENE, similar to the dominant fast directions at stations MKN and MGR. Fast directions at station NBR north of the caldera point NNW, and station SUN in the vicinity of the central craters has a high scatter in $\phi$. Mean directions are summarised in table 7.7. 


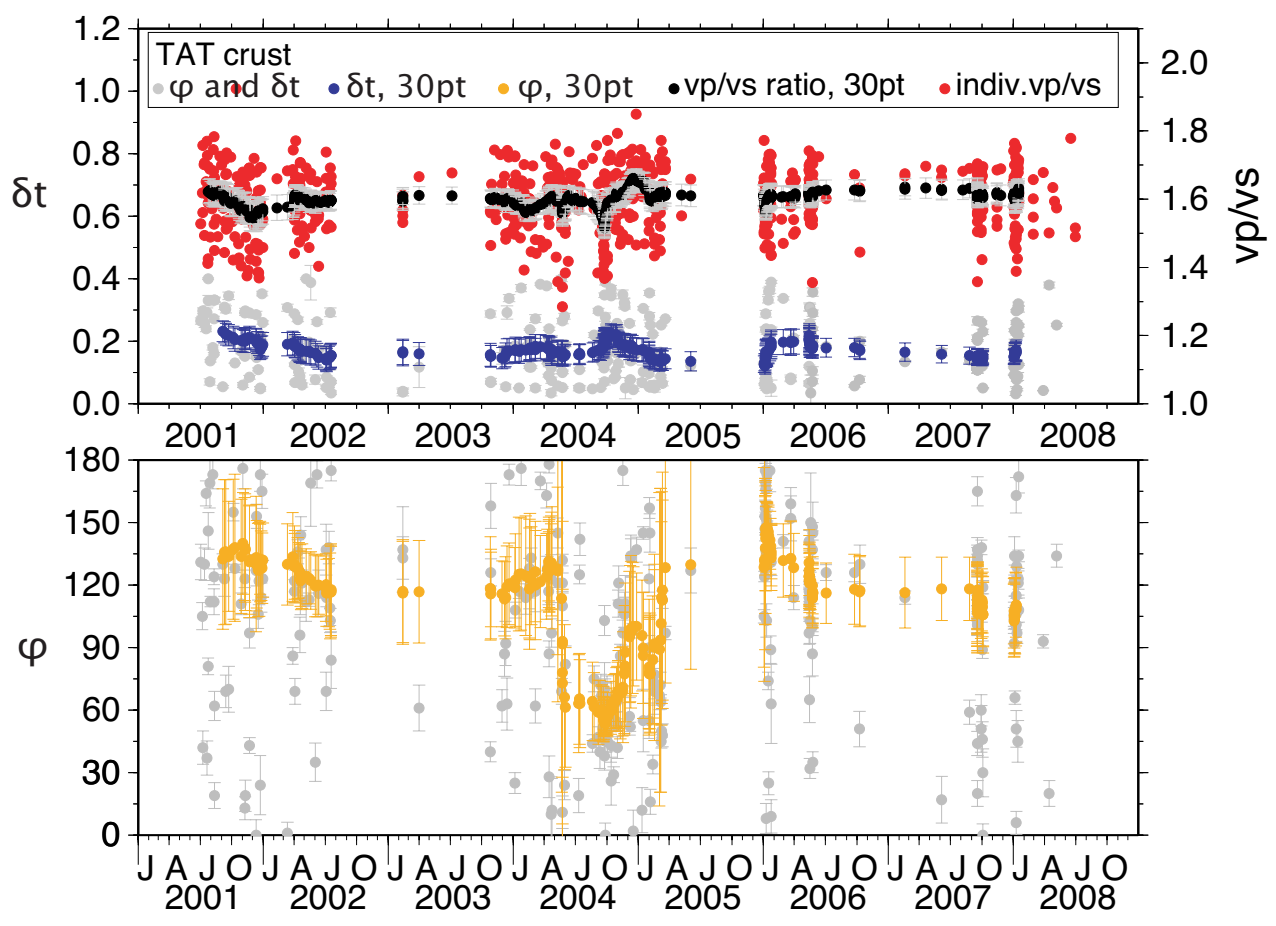

Figure 7.11: Moving means over splitting parameters and $v_{P} / v_{S}$-ratio at station TAT. Grey dots are individual $\phi$ and $\delta t$, orange dots and blue dots are 30 point moving means over $\phi$ and $\delta t$, respectively (all for events shallower than $35 \mathrm{~km}$ ). Red dots are individual $v_{P} / v_{S}$-ratio values and black dots are 30 point moving means over $v_{P} / v_{S}$-ratio. Error bars are $95 \%$ confidence intervals. Note that the error bars on the moving means are strongly correlated, since only every 30 th point is independent.

$V_{P} / V_{S}$-ratio The ratio of the $P$ - to the $S$-velocities generally varies around an average of 1.65 with a standard deviation of 0.09 for all stations and all years (see table 7.8 for station means). Standard deviations on the order of 0.1 can be observed throughout the years at individual stations. Most stations show slightly stronger variations between 2003-2005 than for the other times, similar to the delay times (see figure 7.11).

\subsubsection{Deformation: time series and strain}

Time series and baseline lengths Most stations show a general trend of movement towards the southeast (see figure 6.7). There are only minor deviations from this trend, which are difficult to investigate by just looking at the time series themselves. Instead, focussing on baselines reveals an interesting pattern: Baselines crossing the centre of the caldera connecting station pairs inside as well as outside of the caldera generally shorten. Between May and November 2003, however, an increase in baseline lengths on the order of $5 \mathrm{~mm}$ outside and even more inside the caldera can be observed (figure 7.13(b) and 7.13(a), respectively). Note that Aso GPS data 


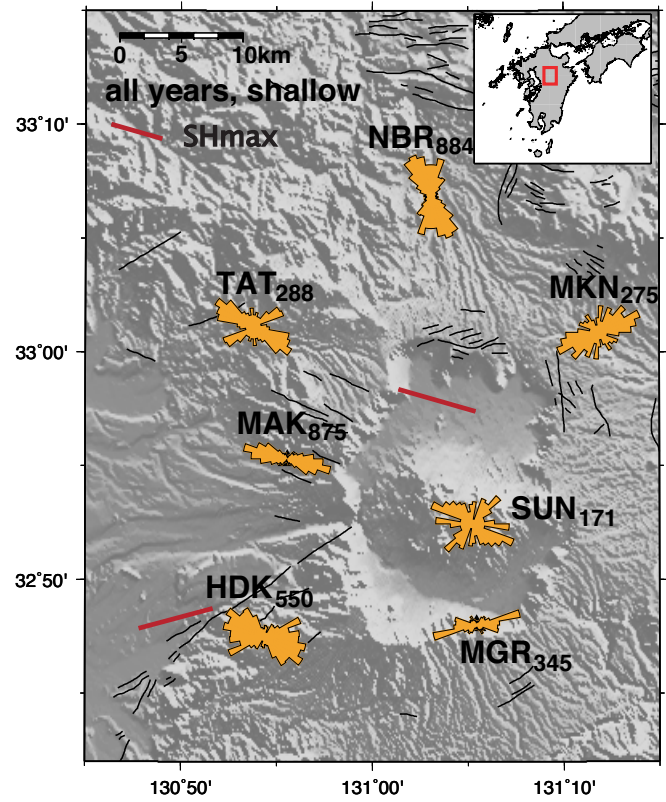

(a) Rose diagrams for shallow events

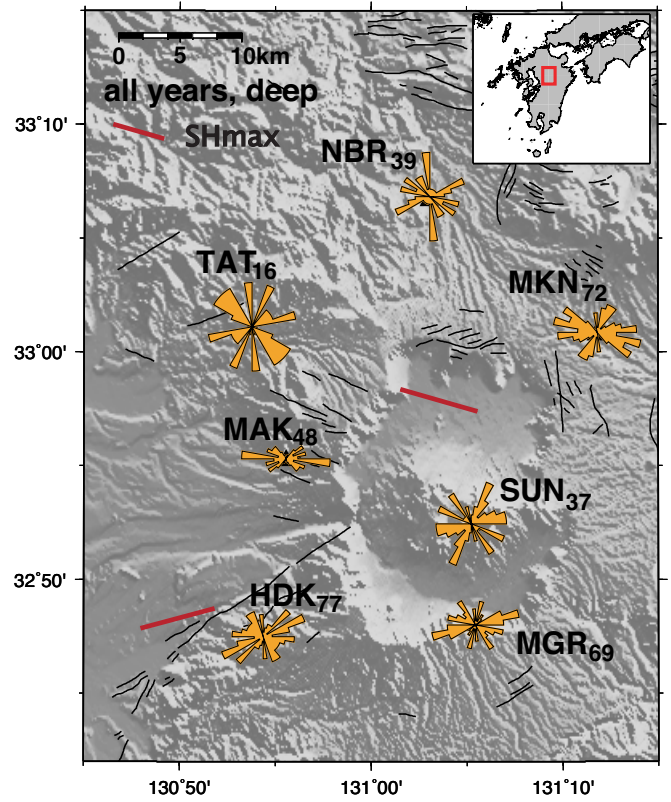

(b) Rose diagrams for deep events

Figure 7.12: Shear-wave splitting fast directions for shallow and deep subset at Aso caldera. Black lines indicate active faults. Red bars are maximum horizontal compressive stress directions after Townend and Zoback (2006). Subscript numbers correspond to number of $\mathrm{A}$ and $\mathrm{B}$ grade measurements at respective station.

\begin{tabular}{|l||l|l|l||l|l|l|}
\hline station & $\begin{array}{l}\text { no. } \\
\text { shallow } \\
\text { meas. }\end{array}$ & $\phi\left(^{\circ}\right)$ & $\delta t$ & $\begin{array}{l}\text { no. } \\
\text { deep } \\
\text { meas. }\end{array}$ & $\phi\left(^{\circ}\right)$ & $\delta t$ \\
\hline \hline HDK & 550 & $-74 \pm 2$ & $0.19 \pm 0.00$ & $77^{*}$ & $88 \pm 14$ & $0.21 \pm 0.01$ \\
\hline MAK & 875 & $-79 \pm 1$ & $0.22 \pm 0.00$ & 48 & $-85 \pm 5$ & $0.24 \pm 0.01$ \\
\hline MGR & 345 & $84 \pm 2$ & $0.22 \pm 0.01$ & $69^{*}$ & $-88 \pm 9$ & $0.19 \pm 0.01$ \\
\hline MKN & 275 & $63 \pm 5$ & $0.19 \pm 0.01$ & $72^{*}$ & $86 \pm 8$ & $0.19 \pm 0.01$ \\
\hline NBR & 884 & $-13 \pm 1$ & $0.16 \pm 0.00$ & $39^{*}$ & $-59 \pm 29$ & $0.24 \pm 0.01$ \\
\hline SUN & $171^{*}$ & $-71 \pm 14$ & $0.20 \pm 0.01$ & $37^{*}$ & $62 \pm 16$ & $0.23 \pm 0.02$ \\
\hline TAT & 288 & $-70 \pm 5$ & $0.18 \pm 0.01$ & $16^{*}$ & $-51 \pm 57$ & $0.19 \pm 0.03$ \\
\hline
\end{tabular}

Table 7.7: Summary of fast directions and delay times with standard errors for shallow and deep events at Aso caldera. A* denotes high scatter or bimodal distribution of $\phi$ for that subset and station and thus meaningless mean value. 


\begin{tabular}{|l||l|l|l|l|}
\hline station & mean $v_{P} / v_{S}$ & standard deviation & minimum value & maximum value \\
\hline \hline HDK & 1.64 & 0.08 & 1.20 & 1.95 \\
\hline MAK & 1.67 & 0.09 & 1.31 & 2.11 \\
\hline MGR & 1.65 & 0.09 & 1.26 & 1.91 \\
\hline MKN & 1.63 & 0.09 & 1.27 & 1.98 \\
\hline NBR & 1.67 & 0.08 & 1.21 & 2.09 \\
\hline SUN & 1.66 & 0.14 & 1.21 & 2.08 \\
\hline TAT & 1.60 & 0.09 & 1.29 & 1.92 \\
\hline
\end{tabular}

Table 7.8: Mean, standard deviation, minimum and maximum of $v_{P} / v_{S}$-ratio for 20012008 at Aso caldera. Note that all values lower than 1.2 and higher than 2.2 (46 out of 3849 total or $1.2 \%$ ) are in most cases due to erroneous picks and have been removed

were received without error bars. With the assumed errors from mean standard deviations of $3 \mathrm{~mm}$ on the horizontal components, we get an estimate for standard deviation of each baseline component of $4.2 \mathrm{~mm}$, giving overall baseline errors on the order of $6 \mathrm{~mm}$, which indicates a significant change of baselines inside of the caldera. We can hence infer that the change in length outside of the caldera at the same time could be a real variation, even though it is below the statistical significance level when the baseline is considered independently.

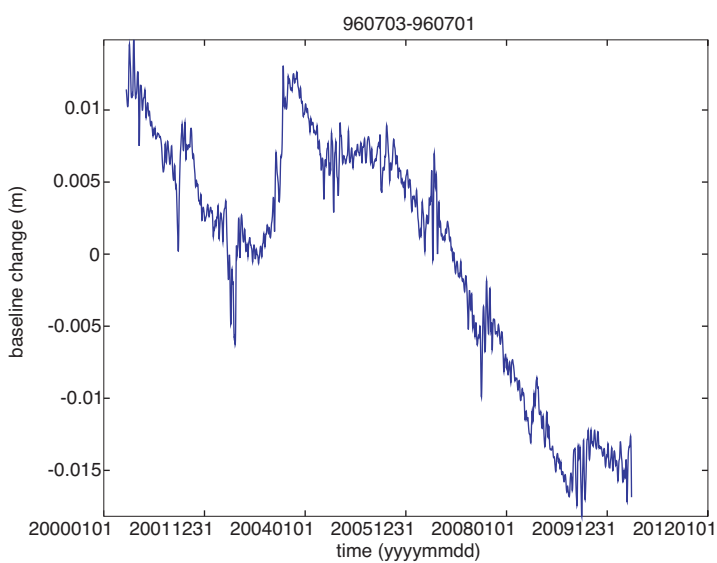

(a) $960703-960701$

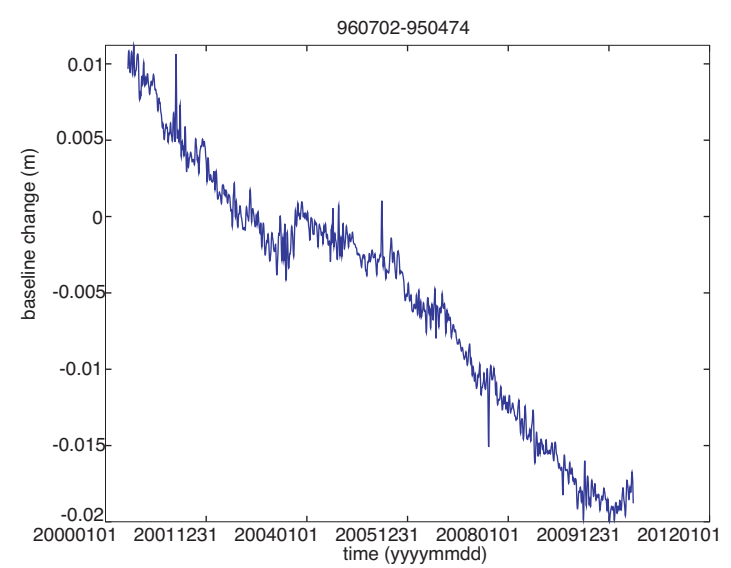

(b) $960702-950474$

Figure 7.13: Baseline changes for station pairs 960703-960701 (stations inside of the caldera, refer to figure 6.5 for station locations) and 960702-950474 (stations outside) at Aso caldera. Both pairs show extension in 2003 opposing a general trend of compression.

2D Strain Figure 7.14 shows the calculated strain fields for the different periods. We do not show $49 \mathrm{~km}$ (seven rows of grid cells) in the south, as the caldera is the main area of interest. Looking at the broad picture, the strain field in general shows 
shortening axes oriented roughly east-west ${ }^{28}$, especially for the long time periods ${ }^{29}$ I and VI. In contrast, the shortest time periods IV and V exhibit a very "patchy" strain field where it is hard to determine trends. Yet, for period IV the caldera appears to be an extensional feature. In all the other periods, the caldera strongly stands out. Compressional areal dilatation ${ }^{30}$ of up to $\sim 1.6 * 10^{-6} \pm 2 * 10^{-8}$ is dominating in periods I, III and VI. In contrast, period II is dominated by extension of $\sim 8.4 * 10^{-7} \pm 1 * 10^{-8}$ in the caldera and shows larger deviations in shortening axes from the general east-west trend: Instead, shortening directions seem to radiate away from the caldera.

\subsection{Gisborne}

\subsubsection{Shear-wave splitting}

2002-2005 1842 earthquakes in total yielded 1710 A or B grade splitting measurements on two stations. In 2004 another station was set up in this area, thus a higher cutoff magnitude applies for the time pre-2004, as with better station coverage smaller earthquakes can be detected and localised. To avoid magnitude comleteness effects on apparent temporal variations we hence excluded events with magnitudes lower than 3.0 at all times. This leaves a total of 196 earthquakes giving $443 \mathrm{~A}$ and B grade splitting measurements (see figure 7.15a). Their characteristics are summarised in table 7.9.

\begin{tabular}{|l||l|l||l||l|l|}
\hline & minimum & maximum & & minimum & maximum \\
\hline \hline latitude $\left(^{\circ}\right)$ & -39.29 & -37.81 & longitude $\left(^{\circ}\right)$ & 177.44 & 178.57 \\
\hline distance $(\mathrm{km})$ & 2.95 & 148.97 & depth $(\mathrm{km})$ & 5 & 85.29 \\
\hline magnitude & 3.0 & 4.8 & $\begin{array}{l}\text { angle of inci- } \\
\text { dence }\left(^{\circ}\right)\end{array}$ & 3.4 & 27.5 \\
\hline$\delta \mathrm{t}(\mathrm{s})$ & 0.02 & 0.95 & $\begin{array}{l}\text { standard devia- } \\
\text { tion of } \delta \mathrm{t}(\mathrm{s})\end{array}$ & $<0.002$ & 0.12 \\
\hline$\phi\left(^{\circ}\right)$ & -89 & 90 & $\begin{array}{l}\text { standard devia- } \\
\text { tion of } \phi\left(^{\circ}\right)\end{array}$ & 0.25 & 23.25 \\
\hline
\end{tabular}

Table 7.9: Summary of parameters from shear-wave splitting from the Raukumara peninsula from 2002-2005

2009-2010 For 2009-2010, 900 A and B grade measurements from 251 earthquakes with magnitudes higher than 3.0 were obtained on three stations from a total of

\footnotetext{
${ }^{28}$ SSPX does not provide error bars for strain axes direction estimates. Error bars will most likely be several degrees. Error fields for the dilatation magnitudes are shown in appendix A.

${ }^{29}$ For definition of periods see section 6.2 .2 , table 6.6

${ }^{30}$ GPS data were provided without error bars. All errors reported here are derived from the overdetermination of the inverse problem in SSPX, as discussed in section 5.2.3.
} 

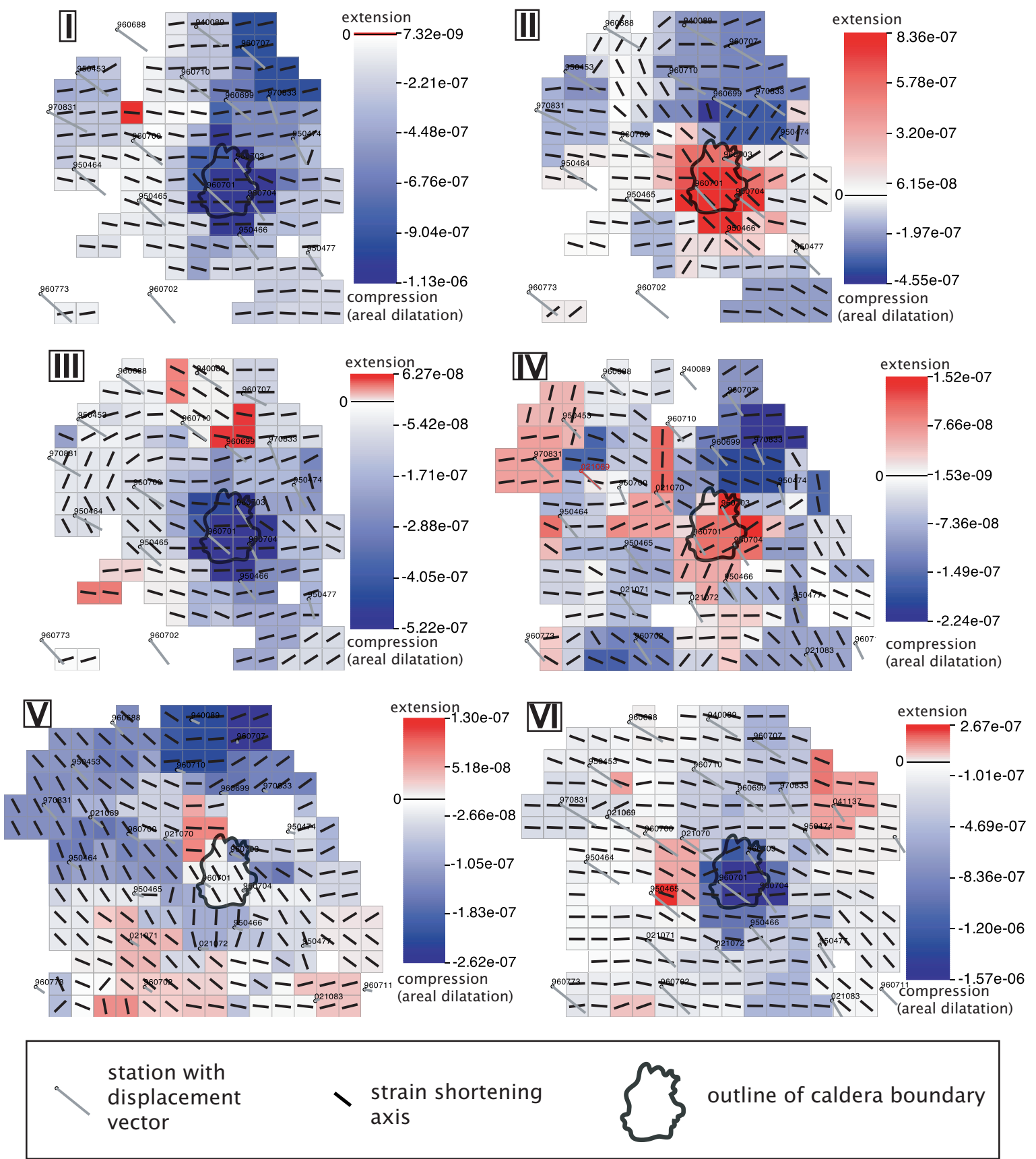

Figure 7.14: Strain field for all periods at Aso caldera. Colours represent areal dilatation, black bars are shortening axes, grey bars and small numbers are displacement vectors and station IDs. The caldera boundary is outlined in black in the centre of each plot and the roman number indicates the respective time period. Note that the caldera stands out in most periods, and that especially the shorter period IV is noisier than the rest. For error fields see appendix A. 


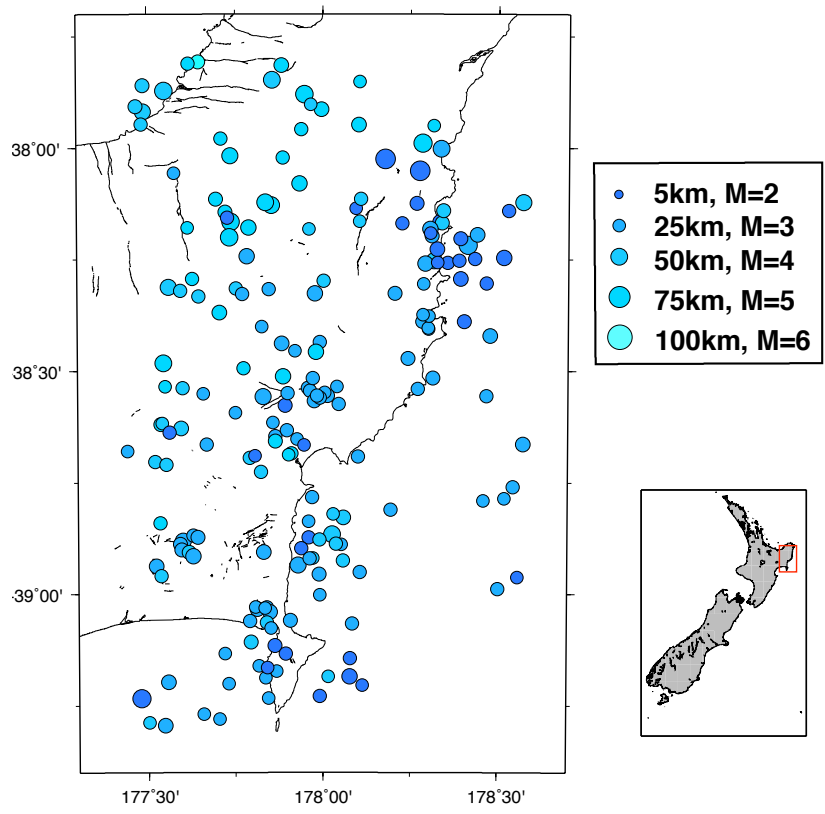

(a) $2002-2005$

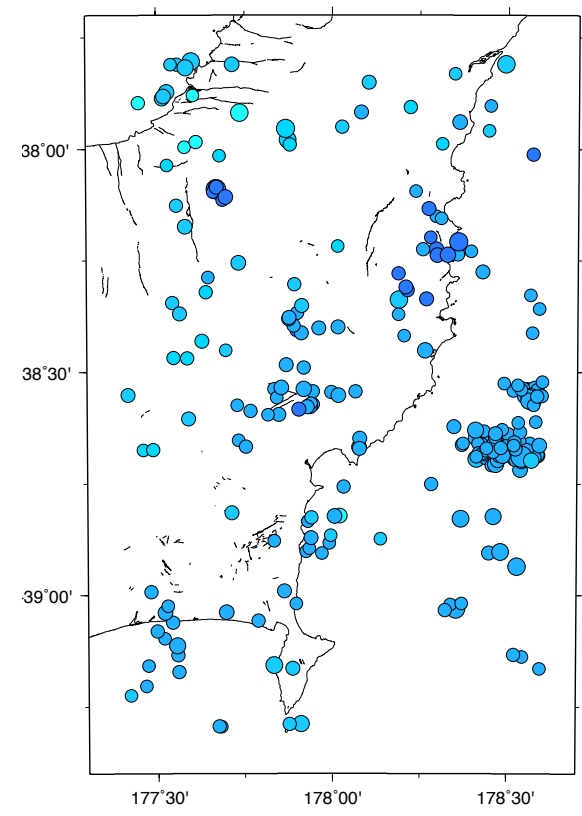

(b) 2009-2010

Figure 7.15: Earthquakes with A or B grade splitting measurements for the Raukumara Peninsula. Results from shear-wave splitting are shown in figure 7.11.

2892 earthquakes, as seen in figure 7.15b. Their results can be seen in table 7.10. Although at this time the cutoff magnitude can be expected to be lower than 3.0, we used the same magnitude threshold as for 2002-2005 to make the results comparable.

Temporal variations We applied moving means to all data to emphasise trends. Generally, all stations show some changes in $\phi$ and $\delta t$ over the 2002-2005 and the 2009-2010 periods (see figures 7.16 and 7.17). Mean delay times from a 30 point window vary between roughly 0.1 and $0.4 \mathrm{~s}$. Mean fast directions from a 30 point window differ from station to station and show changes of up to $\pm 45^{\circ}$ around the respective mean value. At station KNZ, a relatively sharp change in $\phi$ accompanied by a slight increase in $\delta t$ can be observed at the time of the first slow slip event (October 2002). However, except for another slight change of the ten point moving means of $\delta t$ around the 2004 there are no similar variations at times of other slow slip events or at any of the other stations, and the change becomes less clear if we only look at splitting results from the best filter fb1.

Table 7.11 summarises mean fast directions and delay times over the entire periods 2002-2005 and 2009-2010. Both stations KNZ and PUZ, which have been operating in 2002-2005 as well as in 2009-2010, show no major changes in $\delta t$. Fast directions at station PUZ change slightly from $5^{\circ} \pm 3^{\circ}$ to $-5^{\circ} \pm 5^{\circ}$ (figure 7.18). $\phi$ at 


\begin{tabular}{|l||l|l||l||l|l|}
\hline & minimum & maximum & & minimum & maximum \\
\hline \hline latitude $\left(^{\circ}\right)$ & -39.30 & -37.80 & longitude $\left(^{\circ}\right)$ & 177.42 & 178.60 \\
\hline distance $(\mathrm{km})$ & 1.75 & 136.84 & depth $(\mathrm{km})$ & 2 & 95.47 \\
\hline magnitude & 3.0 & 5.0 & $\begin{array}{l}\text { angle of inci- } \\
\text { dence }\left(^{\circ}\right)\end{array}$ & 0.8 & 27.5 \\
\hline$\delta \mathrm{t}(\mathrm{s})$ & 0.02 & 0.99 & $\begin{array}{l}\text { standard devia- } \\
\text { tion of } \delta \mathrm{t}(\mathrm{s})\end{array}$ & $<0.002$ & 0.15 \\
\hline$\phi\left(^{\circ}\right)$ & -89 & 89 & $\begin{array}{l}\text { standard devia- } \\
\text { tion of } \phi\left(^{\circ}\right)\end{array}$ & 0.25 & 22.25 \\
\hline
\end{tabular}

Table 7.10: Summary of parameters from shear-wave splitting from the Raukumara peninsula from 2009-2010.

KNZ is constant for both time periods.

Table 7.12 summarises mean fast directions and delay times for the subperiods 2009 (before slow slip), 2010 during slow slip and 2010 after slow slip. The respective rose diagrams can also be seen in figure 7.18. Even though the moving means did not reveal any variations that can directly be linked to slow slip, the mean directions for the different periods change at station PUZ, where the mean $\phi$ shifts from an orientation towards NNE to NNW from 2009 to 2010. The period during slow slip in 2010, however, has a relatively high standard error and the period after slow slip in 2010 exhibits a bimodal distribution and hence a meaningless mean fast direction despite the fact that a change of the mode of $\phi$ can be seen. Similarly, $\phi$ in 2009 at station PUZ shows a high scatter, even though the standard error of the mean fast direction is low. Thus, we cannot be completely sure about the significance of the observed change. Interestingly, while $\phi$ at stations MWZ an KNZ stays constant, the mean $\delta t$ at station KNZ decreases to half its initial value from 2009 to early 2010. However, comparison to the moving means (figure 7.16) suggest that the decrease is not sharp but gradual, and seems to be related to a high in delay times during 2009 rather than a low related to slow slip.

Spatial variations For 2002-2005, stations PUZ and KNZ show distinct fast directions: While the trend at PUZ is roughly north-south, $\phi$ at KNZ is oriented ENE (figure 7.18). For 2009-2010, basically the same pattern can be observed. $\phi$ at PUZ deviates slightly more to the west $\left(-5^{\circ} \pm 5^{\circ}\right)$ from its previous orientation of $5^{\circ} \pm 3^{\circ}$ and is more scattered than for 2002-2005. Fast directions at MWZ and KNZ are parallel to the trend observed at KNZ for the years 2002-2005.

\subsubsection{Deformation: time series and strain}

Time series Raw and filtered time series at station GISB are shown in figure 7.19. The mean uncertainties for station GISB are $0.004 \mathrm{~m}, 0.003 \mathrm{~m}$, and $0.010 \mathrm{~m}$ in 

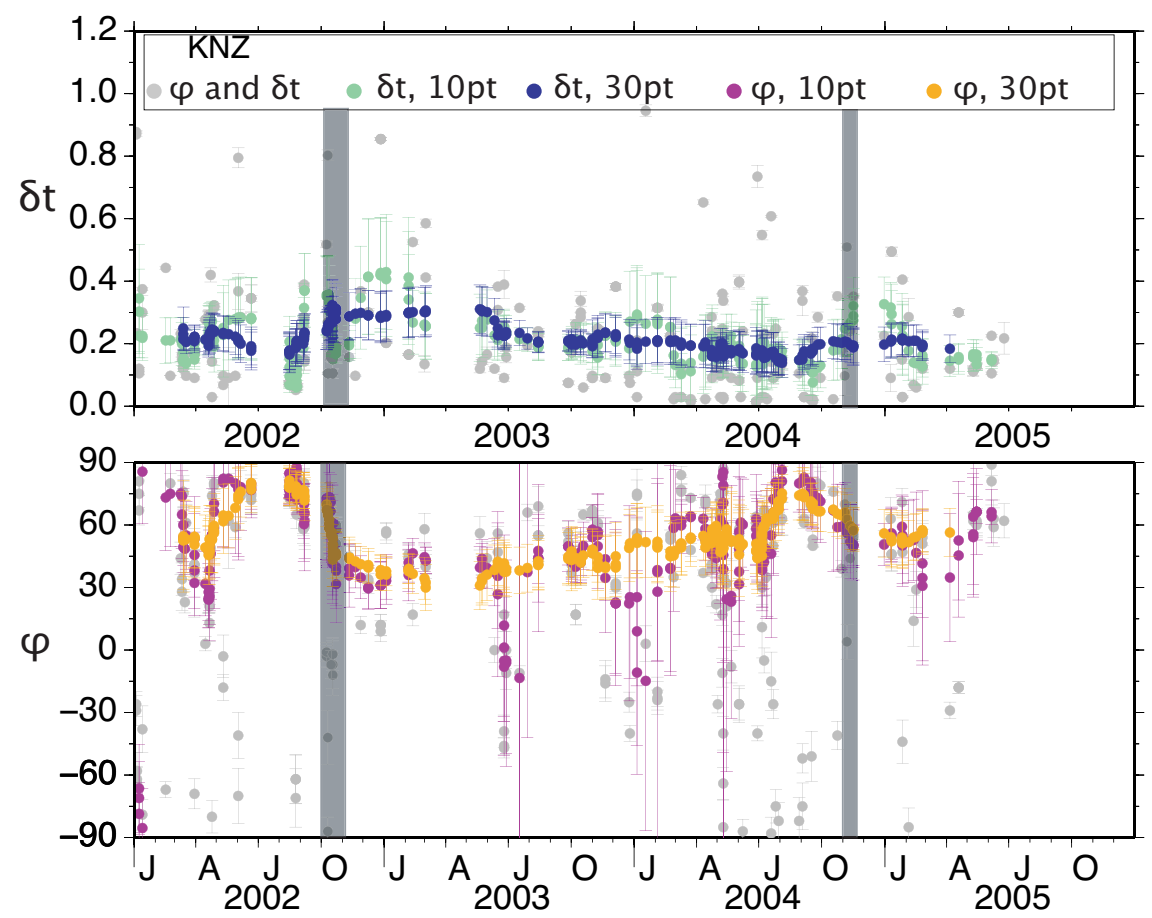

(a) 2002-2005

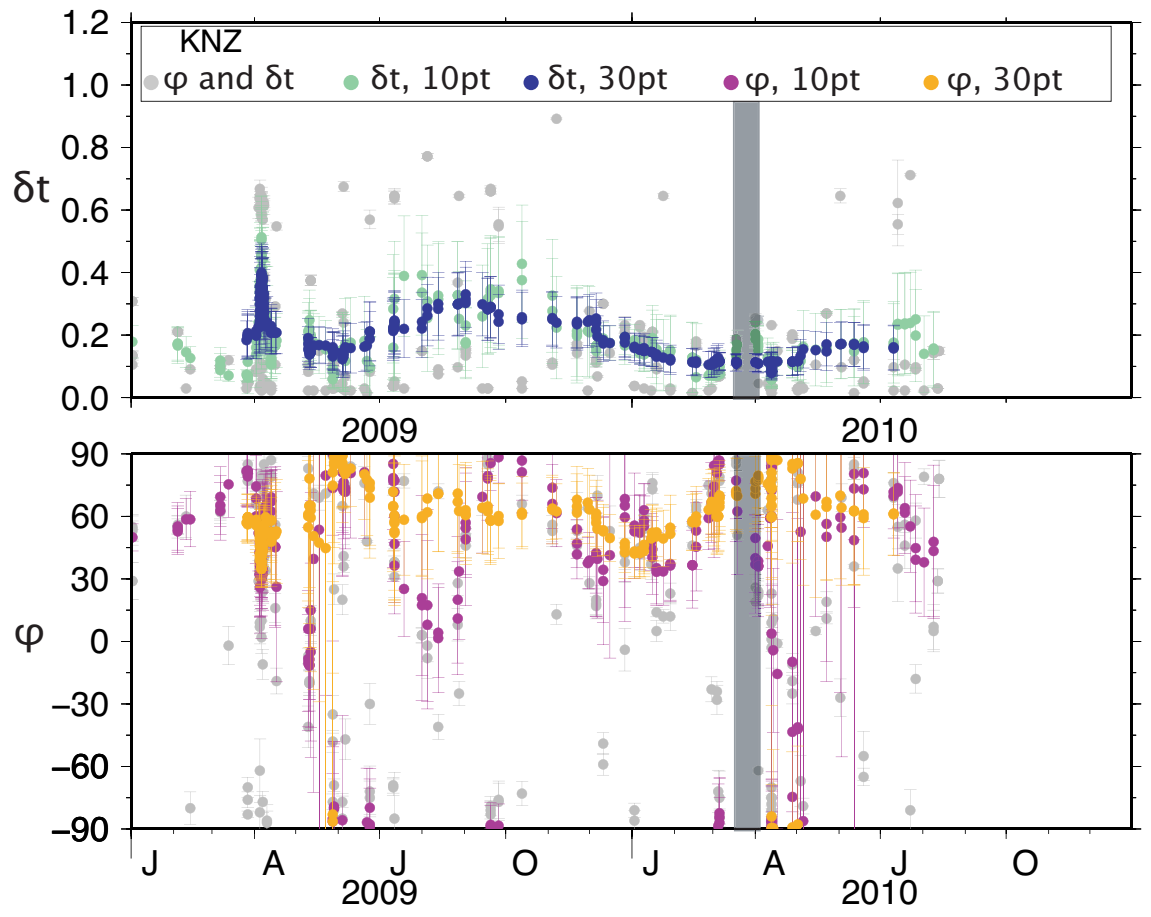

(b) 2009-2010

Figure 7.16: Moving means over $\phi\left(^{\circ}\right)$ and $\delta t(\mathrm{~s})$ at station KNZ, Raukumara Peninsula. Grey dots are individual measurement results, purple and orange dots are 10 and 30 point moving means over $\phi$, respectively, and green and blue dots are 10 and 30 point moving means over $\delta t$, respectively. Error bars are $95 \%$ confidence intervals. Note that the error bars on the moving means are strongly correlated, since only every 10th or 30th point, respectively, is independent. Grey shaded rectangles indicate periods of slow slip derived from GPS. 


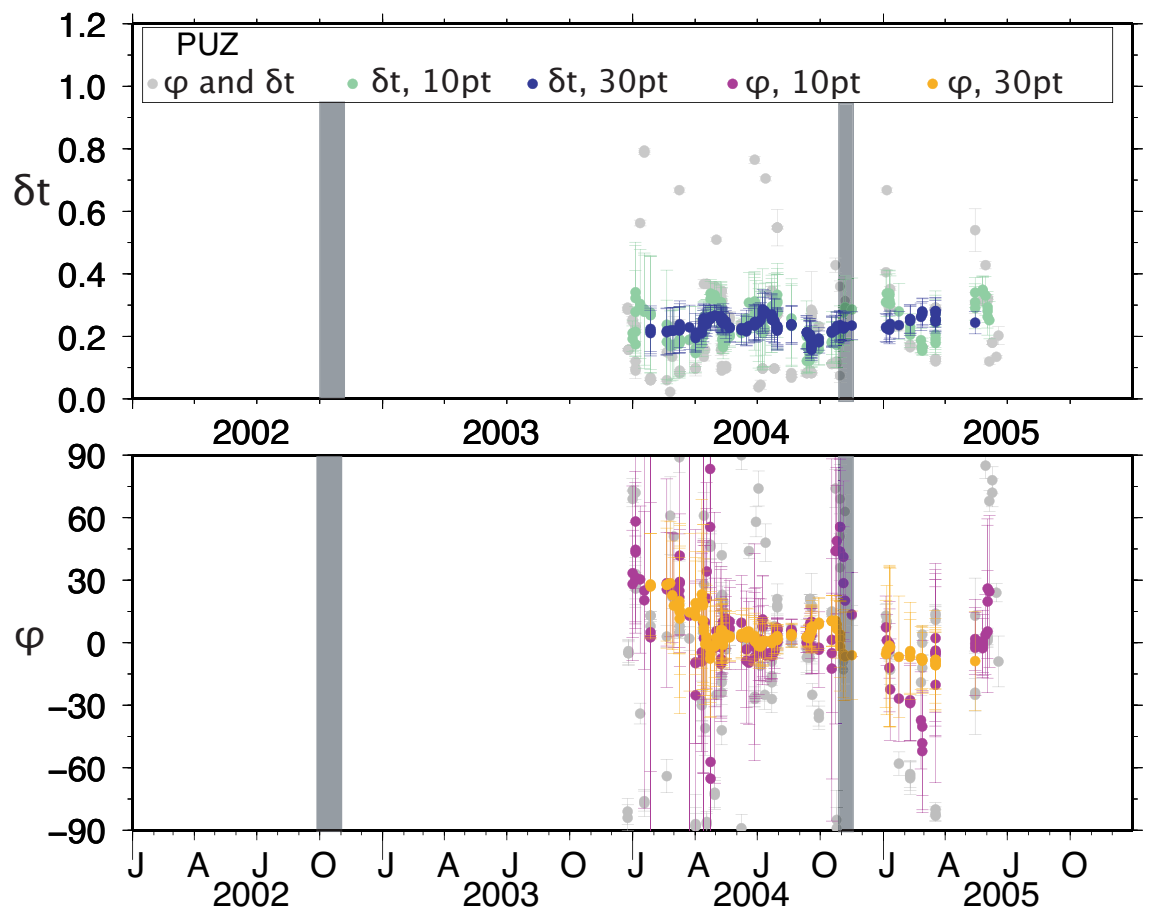

(a) 2002-2005

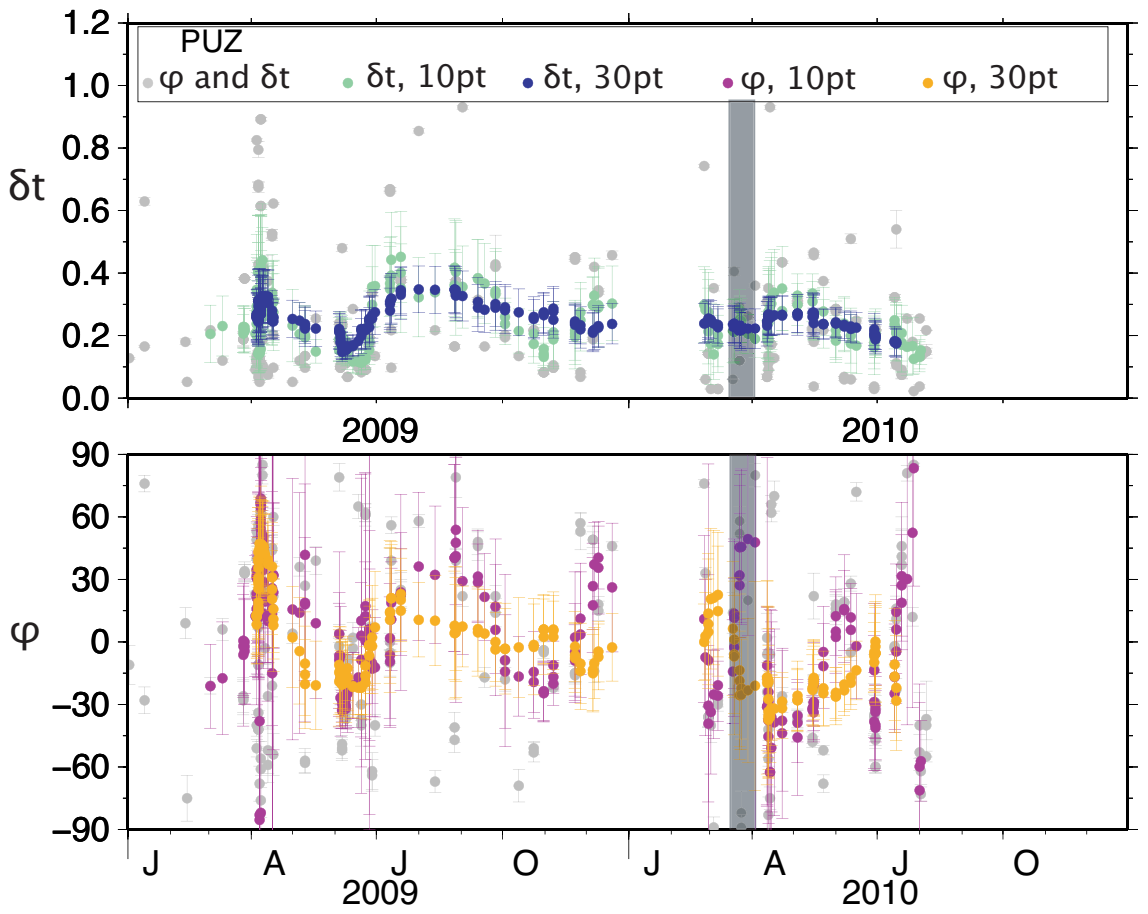

(b) 2009-2010

Figure 7.17: Moving means over $\phi\left(^{\circ}\right)$ and $\delta t(\mathrm{~s})$ at station PUZ, Raukumara Peninsula. Grey dots are individual measurement results, purple and orange dots are 10 and 30 point moving means over $\phi$, respectively, and green and blue dots are 10 and 30 point moving means over $\delta t$, respectively. Error bars are $95 \%$ confidence intervals. Note that the error bars on the moving means are strongly correlated, since only every 10th or 30th point, respectively, is independent. Grey shaded rectangles indicate periods of slow slip derived from GPS. 


\begin{tabular}{|l||l|l|l|}
\hline $\begin{array}{l}\text { station (2002- } \\
2005)\end{array}$ & $\begin{array}{l}\text { no. } \\
\text { meas. }\end{array}$ & $\phi\left(^{\circ}\right)$ & $\delta t(\mathrm{~s})$ \\
\hline \hline KNZ & 275 & $56 \pm 2$ & $0.21 \pm 0.01$ \\
\hline PUZ & 168 & $5 \pm 3$ & $0.24 \pm 0.01$ \\
\hline \hline $\begin{array}{l}\text { station (2009- } \\
2010)\end{array}$ & & & \\
\hline \hline KNZ & 260 & $58 \pm 3$ & $0.20 \pm 0.01$ \\
\hline MWZ & 413 & $60 \pm 1$ & $0.15 \pm 0.01$ \\
\hline PUZ & 227 & $-5 \pm 5$ & $0.25 \pm 0.01$ \\
\hline
\end{tabular}

Table 7.11: Summary of fast directions and delay times with standard errors for all events $\mathrm{M} \geq 3.0$ (see figure 7.15) on the Raukumara Peninsula 2002-2005 and 2009-2010.

\begin{tabular}{|l||l|l|l|}
\hline period & KNZ & MWZ & PUZ \\
\hline \hline 2009 & \multicolumn{4}{|l|}{} \\
\hline \hline no. of measurements & 161 & 269 & 145 \\
\hline$\phi\left(^{\circ}\right)$ & $55 \pm 4$ & $59 \pm 2$ & $7 \pm 6$ \\
\hline$\delta t(\mathrm{~s})$ & $0.24 \pm 0.02$ & $0.15 \pm 0.01$ & $0.26 \pm 0.02$ \\
\hline \hline 2010 during slow slip & \multicolumn{5}{|l|}{} \\
\hline \hline no. of measurements & 68 & 86 & 36 \\
\hline$\phi\left(^{\circ}\right)$ & $64 \pm 7$ & $61 \pm 2$ & $-24 \pm 18$ \\
\hline$\delta t(\mathrm{~s})$ & $0.12 \pm 0.01$ & $0.17 \pm 0.02$ & $0.24 \pm 0.03$ \\
\hline \hline 2010 after slow slip & \multicolumn{5}{|l}{} \\
\hline \hline no. of measurements & 31 & 58 & 46 \\
\hline$\phi\left(^{\circ}\right)$ & $63 \pm 10$ & $62 \pm 4$ & $-25 \pm 7$ \\
\hline$\delta t(\mathrm{~s})$ & $0.16 \pm 0.04$ & $0.14 \pm 0.02$ & $0.21 \pm 0.02$ \\
\hline
\end{tabular}

Table 7.12: Summary of mean fast directions and delay times with standard errors for all events $M \geq 3.0$ on the Raukumara Peninsula for 2009, 2010 during and 2010 after slow slip. See also figure 7.18. 


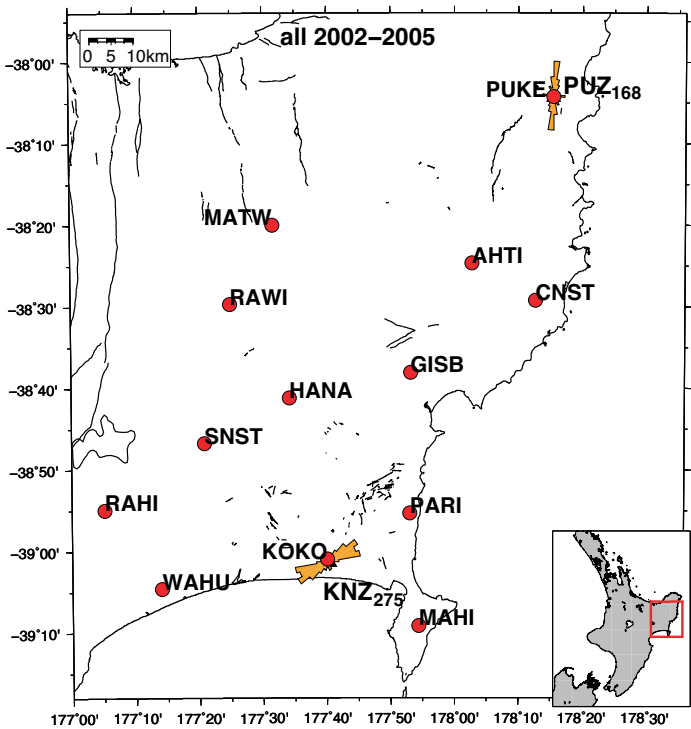

(a) $2002-2005$

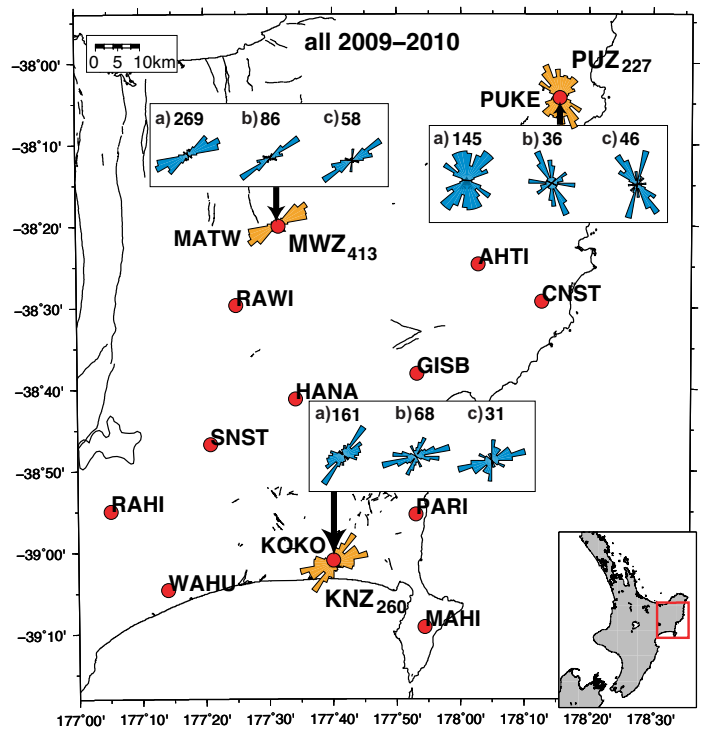

(b) 2009-2010

Figure 7.18: Rose diagrams for Raukumara Peninsula, events with magnitudes higher than 3.0. Subscript numbers next to seismometer names are number of splitting results. Red dots are GPS stations. Black lines on onshore part are active faults. Blue rose diagrams in right hand plot are fast directions for the subperiods before (a), during (b) and after (c) slow slip, with respective numbers of measurements (see also table 7.12).

east, north and vertical components, respectively. The slow slip events are characterised as time spans of days to weeks where the prevalent north-west displacement is reversed with "steps" to the south-east of up to $30 \mathrm{~mm}$. As shear-wave splitting parameters do not show striking changes at the times of the slow slip events, analysis of baselines is omitted for Gisborne.

2D strain Station coverage increased over the years and we were able to calculate the 2D strain field around the last (and largest) slow slip event in 2010 (figure 7.20). There is a distinct change in the strain field during the slow slip event: With the subducting slab striking $\sim 40^{\circ}$ (Reyners and McGinty, 1999), shortening directions change from subperpendicular to the trench (best fit ${ }^{31}$ for all stations with shortening at $108.3^{\circ} \pm 0.1^{\circ}$ ) in 2009 to subparallel to the trench (best fit for all: $209.3^{\circ} \pm 0.1^{\circ}$ ) during the time of slow slip. Furthermore, the region changes from being dominated by compressional areal dilatation of up to $3.94 * 10^{-7} \pm 4 * 10^{-6}$ to extensional dilatation of up to $1.17 * 10^{-6} \pm 1 * 10^{-5}$. The period after the slow slip event is not as clear: Although the best fit for all stations yields shortening direction of $91.8^{\circ} \pm 0.3^{\circ}$ similar to the estimate from 2009 , shortening axes orientations from both before and during the slow slip can be observed in different areas, dominated

${ }^{31}$ Note that errors reported here are solely from the inversion in SSPX. Actual errors are likely to be on the order of degrees if GPS errors are taken into account. 


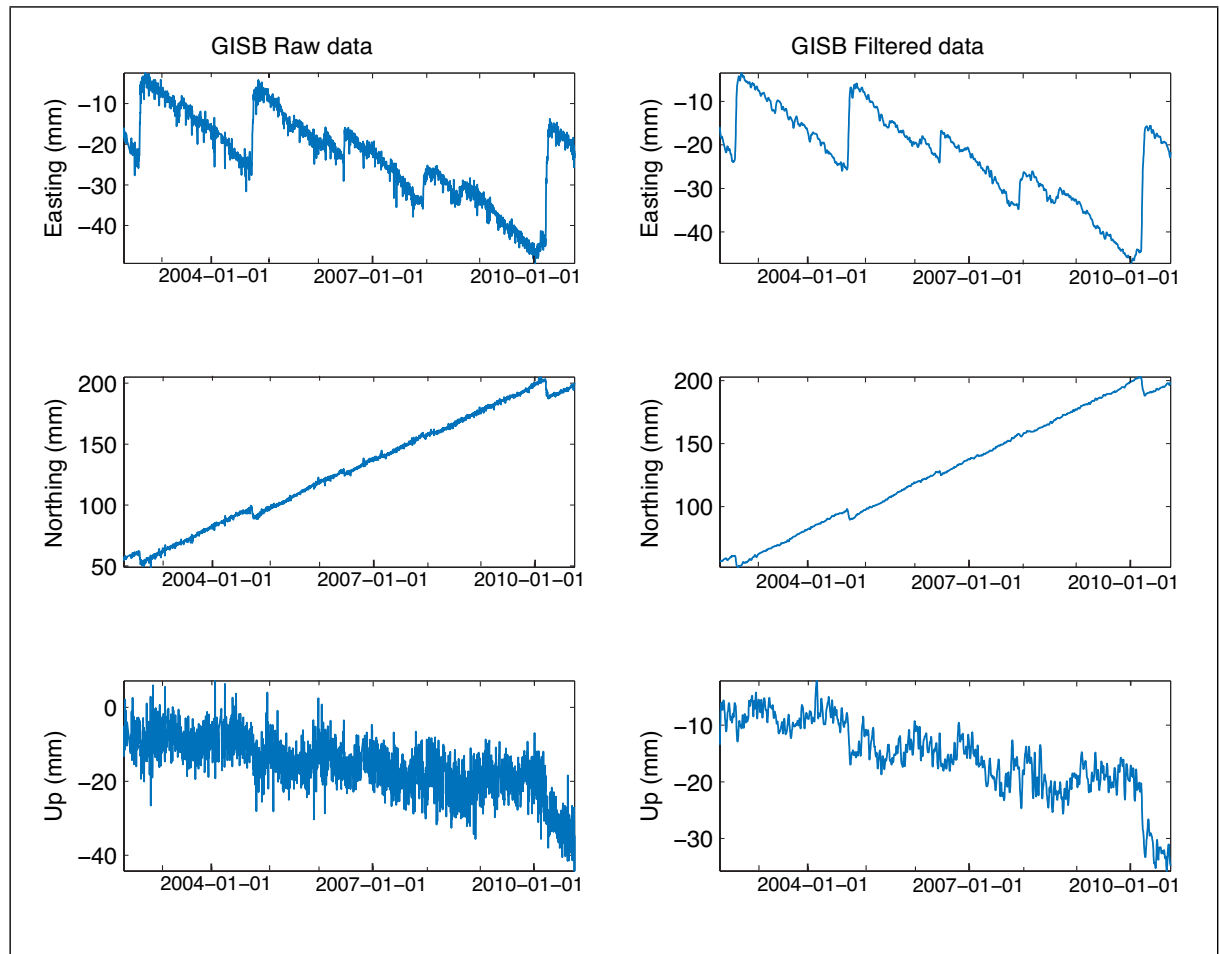

Figure 7.19: GPS timeseries raw and filtered for station GISB, showing several slow slip events.

by compression and extension with absolute dilatation values of up to $\sim 1 * 10^{-7} \pm$ $3 * 10^{-6}$, respectively. 

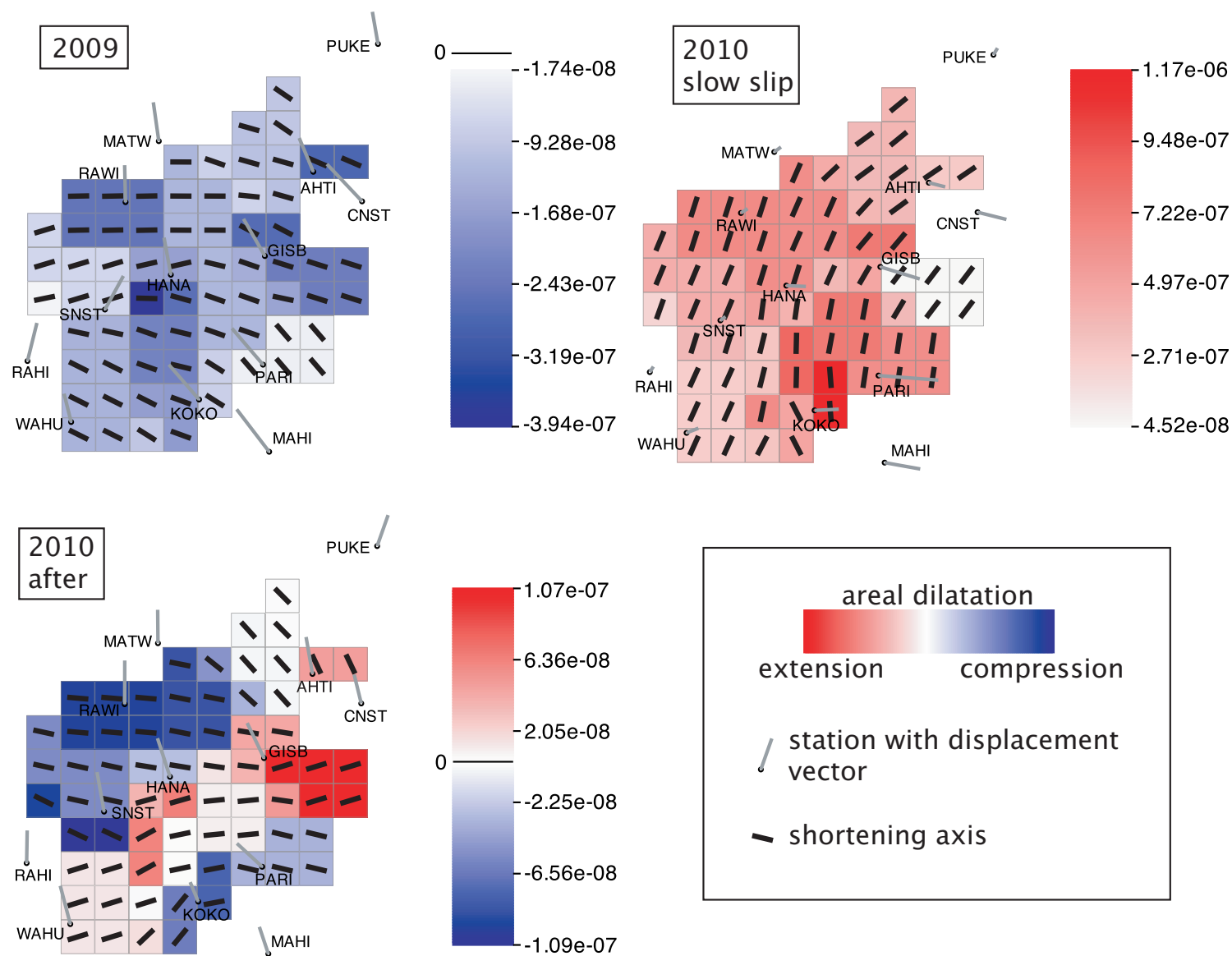

Figure 7.20: Strain field for Raukumara peninsula, 2009 - 2010. Colours indicate areal dilatation, black bars are shortening axes, grey bars and associated names are stations with displacement vectors. For error fields see appendix A. 


\section{Discussion}

To explain the different degrees of detail and analysis steps in the following discussion it is important to make a remark about the development of this project: During the early stages the focus was only on lake Taupo. The initially observed strong deformation seemed ideal to be compared to shear-wave splitting. Accordingly analysis steps in many different directions were undertaken, during which it became clear that the area might not be suitable to answer the questions we were trying to answer in the given timeframe. Yet we think that it is important to give an overview over the different methods we tested. Thus the majority of the analyses we undertook for data from Taupo caldera are explained in the following discussion, even though we do not provide a detailed interpretation for our observations. To get a better constraint on the amount of displacement/strain necessary to cause potential time varying seismic anisotropy we decided to start a small pilot project on the slow slip events at the Hikurangi subduction margin, which eventually became part of this thesis. According to our information shear-wave splitting in relation to slow slip has not been investigated before, and the conditions seemed promising in terms of a potential correlation between GPS data and shear-wave splitting parameters in time. However, the shear-wave splitting parameters did not reveal a strong time variation that could be related to slow slip in a straightforward way. Since the setting close to the subducting slab makes it difficult to entangle source and path effects on splitting parameters, it became clear that we would not be able to present a complete analysis within the time left for this project. Instead, the regional focus was shifted again: In a previous study by Savage et al. (2008), data from Aso caldera had shown a strong time variation in $\phi$ that yet remained to be analysed in detail. As explained in the sections below, these data gave the most interesting results in this study.

Consequently, the discussion of results from lake Taupo is detailed but without strong interpretation. For reasons of immediate comparison of the volcanic environments, Aso caldera is discussed subsequently. Finally, the results from the Raukumara peninsula are summarised shortly. One should keep in mind the pilot character of this part of the project and the time constraints.

\subsection{Taupo caldera}

\subsubsection{Influences on shear-wave splitting results}

Testing of different filters The automatic splitting technique gives up to three results per event-station pair, according to the three best filters (fb1, fb2 and fb3) for each seismogram. These three pairs of splitting parameters can be very similar but may also show significant differences. The question arises if all three of them should be taken into account for the final interpretation or if only one of them, in general the result from the best filter, should be used. In case of lake Taupo, using only the 
best filter gives only a low number of shear-wave splitting parameters. Including the results from the second and third best filters provides approximately three times this number of measurements. If results for all three filters are similar, then including all of them should for example accentuate the (probably true) fast direction. However, if the results differ from each other by a certain amount, scatter of fast directions will be higher. If the difference in $\phi$ does not result from actual structure but from processing, this is a desired effect, as "stable" fast directions are enhanced whereas "unstable" (i.e. artifact) directions get averaged out.

To account for the fact that different results from different filters might depict actual geology, due to different frequencies "seeing" different anisotropic structures for example, we tested the influence of different filters. Figure 8.1 shows diagrams of dominant frequency of each filtered seismogram versus various parameters of the corresponding earthquake/splitting result. The plots on the left-hand side include all results from the three best filters, whereas the right-hand side only takes the results from the best filter into account. First, it appears that dominant frequencies for only fb1 usually seem to be slightly lower than for fb2 and fb3. Hence it is likely that the best filter has a lower highcut than the other two. Apart from this difference, the patterns are very similar for the two configurations (fb1 vs. fb1-3). There is no dependence of any parameter on dominant frequency that could not be explained by the fact that higher frequencies attenuate more, and hence do not dominate seismograms from earthquakes with great depths or large event-station distances. Similarly, seismograms that are dominated by high frequencies will have travelled shorter raypaths and hence generally show shorter delay times. Thus we infer that there are no anisotropic structures to which waves with different frequencies would be sensitive. 


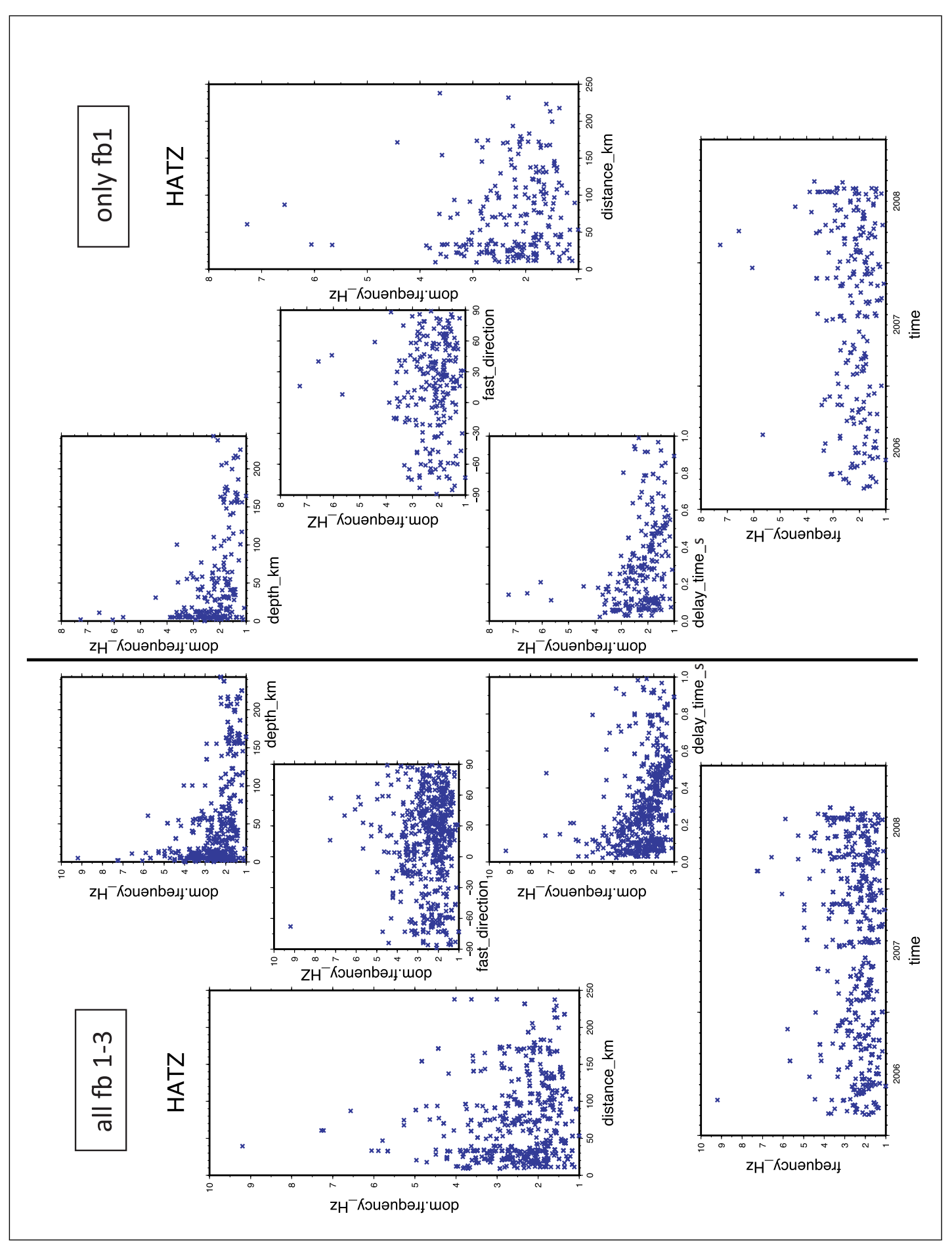

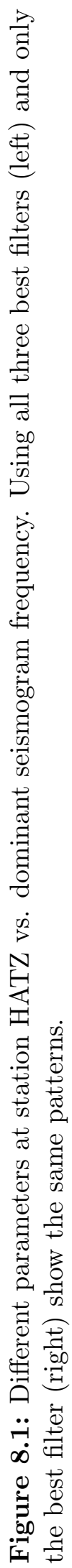


Furthermore we tested the effect of the filters on the moving mean. The lowest plots in figure 8.1 show that there are no major changes in frequency over time. Applying a ten point moving mean results in an apparently higher scatter and more variation for all three filters due a higher number of different measurements (figure 8.2). Again, if the results for one event from $\mathrm{fb} 1, \mathrm{fb} 2$ and fb3 differ from each other, they should average out in the end, whereas "stable" results should be accentuated. Indeed, as we compare moving means for the same earthquakes for only fb1 (ten point moving mean, i.e. ten different earthquakes per mean point) versus fb1-3 (30 point moving mean, i.e. approximately ten different earthquakes per mean point ${ }^{32}$ it can be seen that they basically image the same variations, apart from a higher data density for the configuration where all three results are used (see figure 8.2).

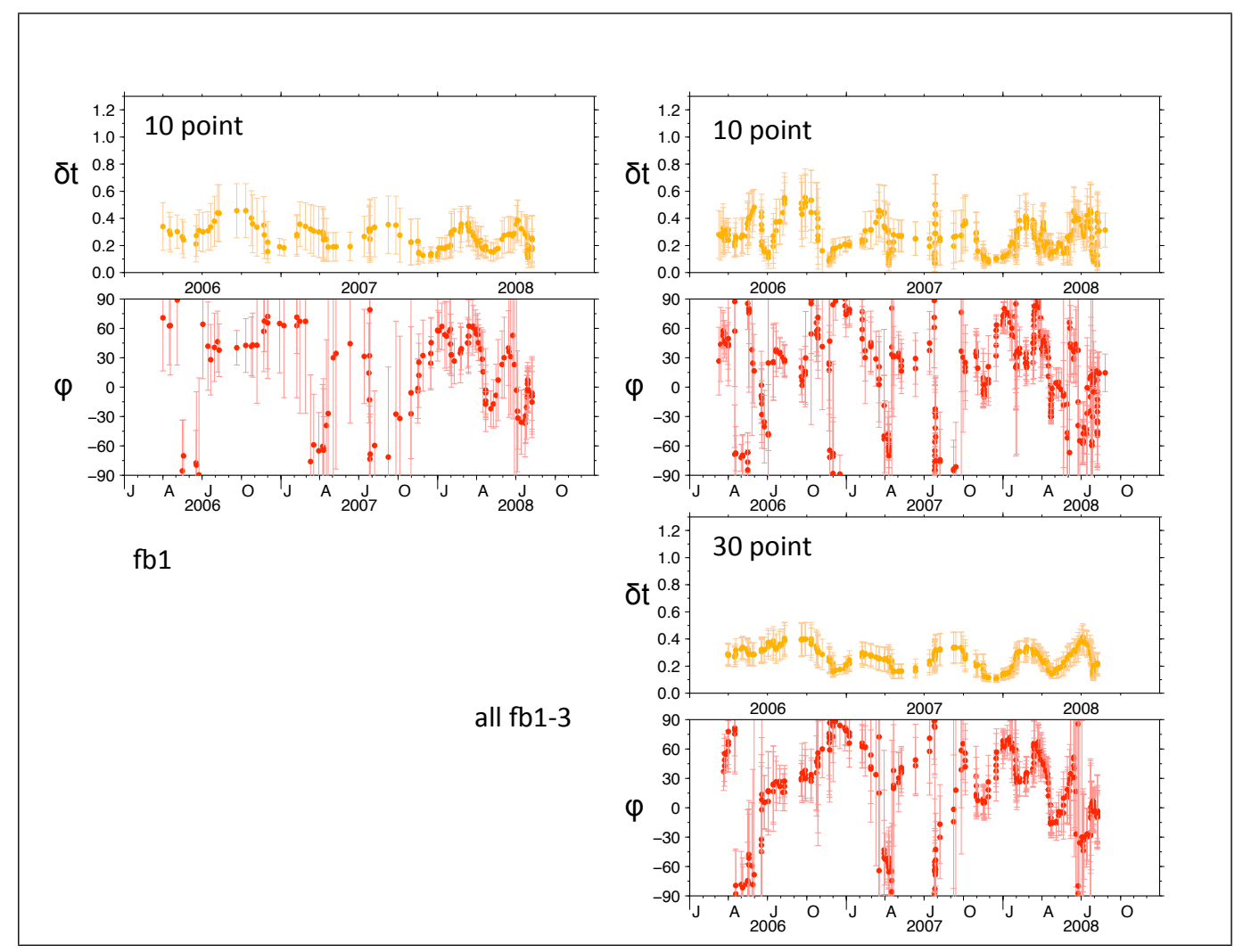

Figure 8.2: Comparison of moving means over results from only the best filter (left) and all three filters (right) for station HATZ. Using a ten point moving mean on only the best filter differs slightly from a ten point moving mean over all three filters, but shows very similar results to using a 30 point moving mean over all three filters. Error bars are $95 \%$ confidence intervals.

It can be concluded, that there are no systematic differences between the results from the three best filters and that it is very unlikely that they sample different

${ }^{32}$ This calculation is based upon the assumption that in average all earthquakes yield three best filters and three measurements 
anisotropic structures. Hence, the results from all three best filters are used for lake Taupo. Furthermore, apparent temporal variations resulting from the processing can be excluded. However, we cannot rule out that changing earthquake locations cause apparent temporal variations in shear-wave splitting parameters.

Dependence of $\delta t$ and $\phi$ on earthquake parameters Shear-wave splitting results can be influenced by the source, the ray path and the receiver area for each earthquake. This study aims to detect possible variations in time in the area around Taupo caldera, i.e. the receiver area. Hence we have to exclude source and path effects. As a first step, different parameters were plotted against each other at each station. Figure 8.3 shows some example plots for two stations and different parameter pairs.

There are no systematic changes of most of the parameters in time (figure 8.3). Neither is there a strong dependence of fast direction or delay time on for example event depth, angle of incidence, or backazimuth. The range of fast directions observed for events deeper than $\sim 50 \mathrm{~km}$ at any given depth seems lower than for shallow events at station HATZ. Similarly, the delay times vary more with higher angles of incidence and with shallower depths, at this station. The fact that we do not see a very well-defined relation between angle of incidence and delay time as well as depth and delay time at most other stations suggests that the observed anistropy is mostly of shallow origin. However, we cannot completely rule out the existence of some dependence for delay times, angle of incidence and depth at the other stations, as they prove to be too noisy to give a large amount of reliable shear-wave splitting results for deep events. We do note the absence of low and high delay times for deep events at both stations shown here, which is also reflected in the generally higher mean delay times from deep events (see table 7.3). This indicates a contribution to the observed splitting by anisotropic regions below $15 \mathrm{~km}$ in addition to the dominant anisotropy from shallower levels and justifies the division of the dataset into shallow and deep events. Furthermore, slight variations of fast directions with backazimuth can be observed. The dependence seems to be irregular between different stations, and we cannot link the variations to other geophysical or geological observations. It can be inferred that most stations measure shear-wave splitting from slightly different ray paths that could be misinterpreted as temporal variations.

To completely exclude path and source effects, we tried to find repeating earthquakes using the BCSeis (Du et al., 2004). However, as described in further detail in appendix B, we could not successfully extract any suitable repeating events from our catalogue.

Correlation The shear-wave splitting parameters over time at each station were tested for a possible correlation. The parameters were read into MatLab and correlation coefficients were calculated from the covariance matrix $\mathrm{C}$ as 

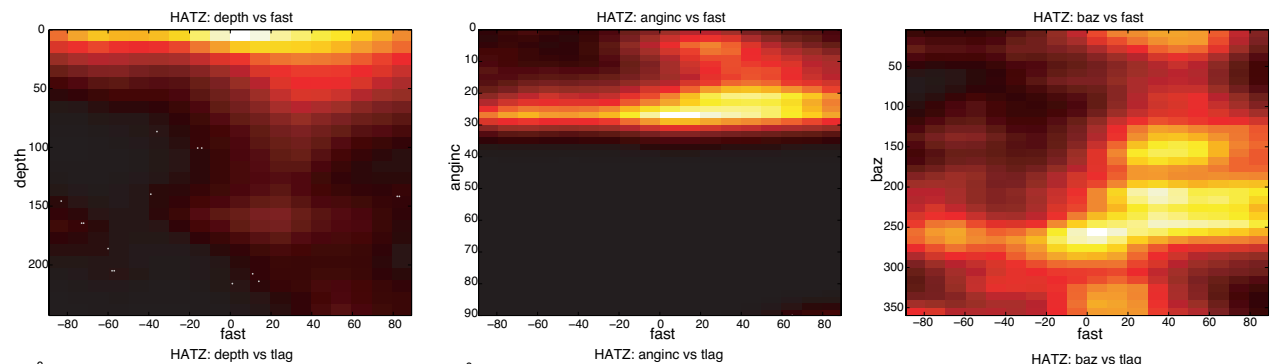

low point

HATZ: anginc vs tha
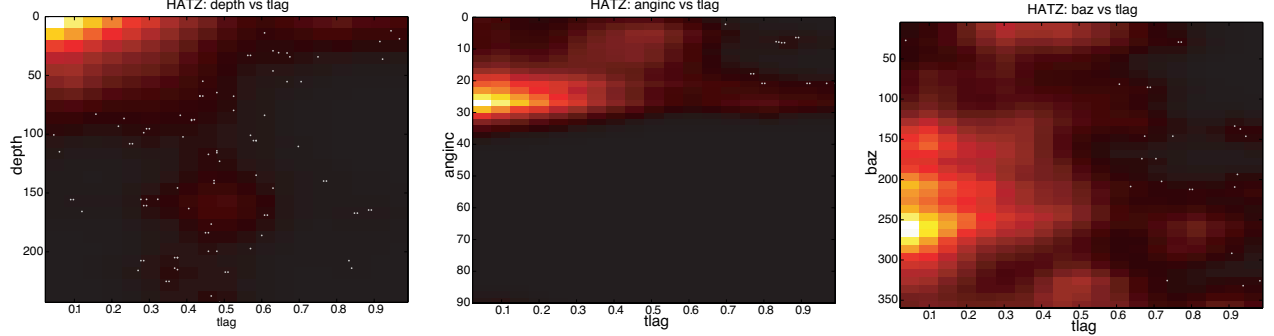

density

(a) HATZ
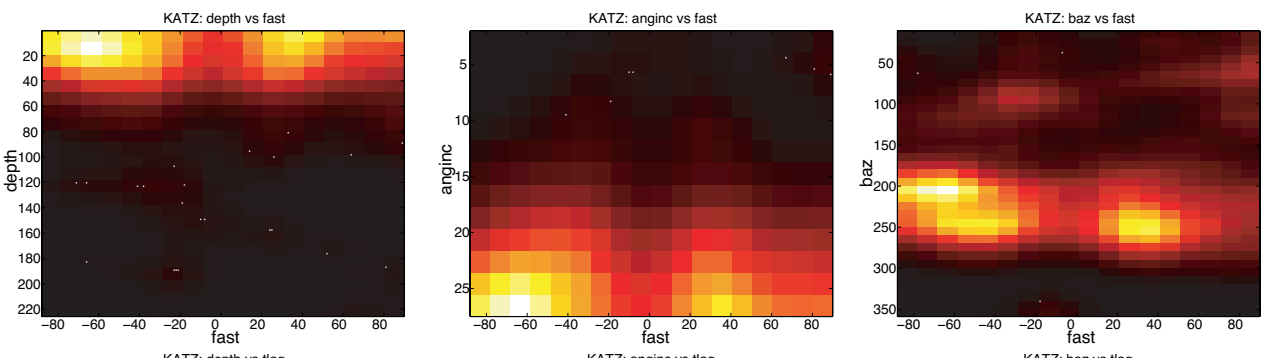

low point density
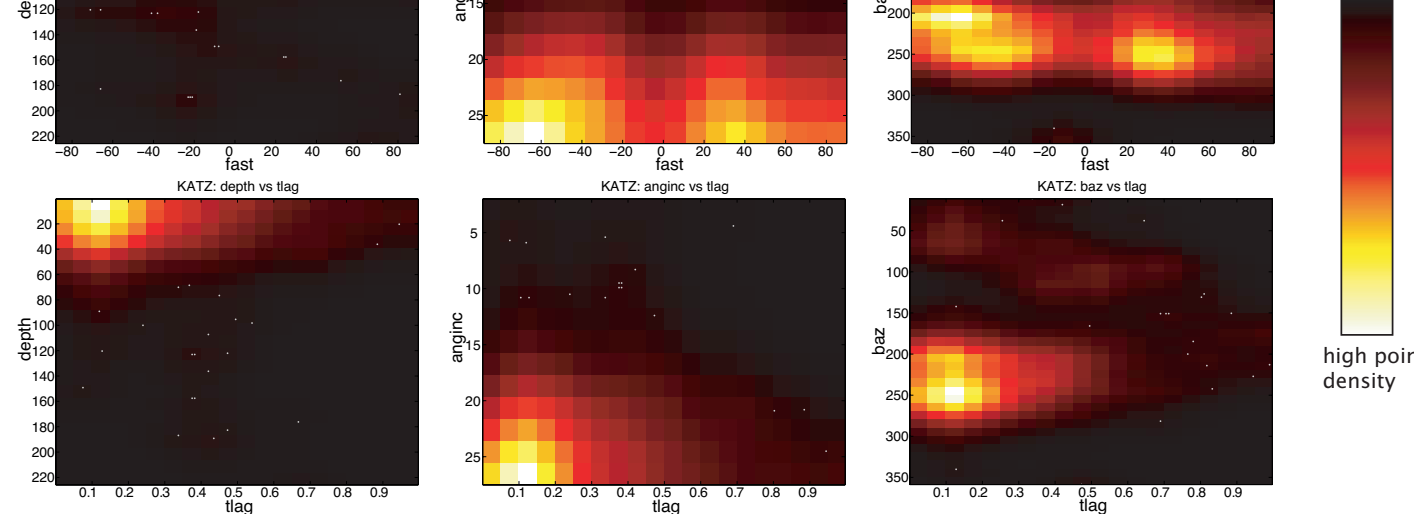

(b) KATZ

Figure 8.3: Dependencies of $\phi$ and $\delta t$ on earthquake parameters at two stations at Taupo caldera. Colours indicate point densities. The white dots that show up in a few plots are artifacts from the density calculation. The upper row is for $\phi\left(\right.$ fast, in $\left.{ }^{\circ}\right)$, the lower row for $\delta t$ (tlag, in seconds). The first column shows depth (in $\mathrm{km}$ ), the second angle of incidence (anginc, in ${ }^{\circ}$ ) and the third backazimuth $\left(b a z\right.$, in $\left.{ }^{\circ}\right)$. Note the different scale for angle of incidence for the two stations. 


$$
R(i, j)=\frac{C(i, j)}{\operatorname{sqrtC}(i, i) * C(j, j)}
$$

Additionally, the significance $p$ of the correlation coefficient was determined using Student's t-test. The $p$-value can take values from 0 to 1 , where low values indicate a low probability that the corresponding correlation coefficient came up by chance.

We put 20 parameters into the correlation matrix. An example of the parameters put into the correlation and the corresponding correlation coefficients and p-values can be found in appendix C. Clearly some of them are not independent, hence care has to be taken when interpreting the results. Out of the 190 different offdiagonal elements of the p-value matrix, approximately one to two thirds are below 0.05 at each station. Although in theory a p-value below 0.05 suggests that the corresponding correlation coefficient is within the $95 \%$ confidence limit of being significant, we cannot simply infer a real correlation of all these parameters. Instead, consulting statistical tables leads to the conclusion that for sample sizes as high as in this case ( $\sim 100$ to over 600 , depending on the station) in fact almost any correlation coefficient will be significant (E. Smith, 2010, pers.comm.). In other words, the more data available the less likely it is that one will find a correlation if the data are not correlated in reality, so even the slightest correlation will be considered significant.

The correlation coefficients were sorted and only the significant ones (within $95 \%$ confidence limit) were taken into account. Some parameter pairs are dependent and yield high correlation coefficients as expected (e.g. latitude or longitude of an event vs. event-station distance: higher than 0.95 at all stations). Interestingly, some of the pairs that are clearly correlated show relatively low coefficients (e.g. the error in the fast direction vs. the minimum of the second eigenvalue of the covariance matrix from shear-wave splitting ${ }^{33}$ : maximum of -0.62 at station POIZ, $\sim 0.4$ at other stations).

The parameters we are most interested in are the fast direction and the delay time. The individual results show a slight correlation of $\delta t$ and depth (at four stations, correlation coefficients between 0.15 and 0.38 ) as well as a slight anticorrelation of $\delta t$ and angle of incidence (at five stations, correlation coefficients between -0.12 and -0.31). Both of the relations are expected, as deeper events tend to have longer raypaths and "see" more anisotropic material along the path. Similarly, low angles of incidence can indicate either deep or very distant events and hence show larger time delays. However, the correlation coefficients for these cases are not particularly high, again indicating mostly shallow anisotropy (see discussion of dependencies above) and/or the presence of vertically oriented cracks ${ }^{34}$. Apart from these expected results, neither of the two parameters show any clear trends of correlation with each other or with other parameters, even if we consider relatively

\footnotetext{
${ }^{33}$ Correlation should arise since the error is computed from the contours of the second eigenvalue.

${ }^{34}$ For the case of vertically oriented cracks causing anisotropy, near-vertical incidence will also see largest amount of anisotropy, i.e. the largest difference between the fast and slow $S$-velocities, or in other words the largest delay times (Crampin, 1978).
} 
low correlation coefficients $(\sim 0.3-0.4)$.

Furthermore, we tested 30 point moving means of $\delta t$ and $\phi$ for correlation with each other. We acknowledge that the statistics we apply might not be exactly valid for this type of data. Nevertheless, the test was meant to serve as an indicator of whether more analyses should be done. The correlation coefficients for the moving means were higher than the ones for the individual data points (up to 0.356 for shallow and 0.46 for all depths at station POIZ, with p-values of 0.0004 and less than $10^{-15}$, respectively).

Neither the differences between data points of $\delta t$ or $\phi$ nor the signum of these differences at each station showed particularly high correlation coefficients for a correlation between $\delta t$ and $\phi$, if we define an arbitrary lower limit of 0.3 for correlation coefficients at $95 \%$ confidence. The same analyses applied to $\delta t$ for different stations did not reveal any striking correlation either.

In summary, correlation coefficients for most parameter pairs show high variations. While coefficients of $\sim 0.95$ exist for pairs that are clearly correlated, other pairs which should show a correlation in theory only yield coefficients of approximately 0.4 or even anticorrelation. Again, drawing a lower limit of 0.3 for correlation coefficients (for p-values lower than 0.05) does not reveal any systematic patterns. Parameter pairs that show a coefficient above this limit at one station might be below at another one or even show anticorrelation at a third station. Thus, even if there might be significant correlations, we do not interpret them. However, we conclude that possibly local processes govern the shear-wave splitting results and no regional trend of correlation can be observed for Lake Taupo from 2006-2008.

Fast directions, stresses and structure The mean fast directions at lake Taupo reported in table 7.3 are only meaningful for stations where scatter is low, the number of measurements is relatively high and if there is no bimodal distribution of fast direction (see table 7.3). Taking only into account those stations (ARAZ, HATZ, POIZ, RITZ), the shallow mean estimate of $37 \pm 3^{\circ}$ (see figure 7.6) is perpendicular to the geodetically determined extension direction of $128 \pm 11^{\circ}$ (Darby et al., 2000). This value lies within the range of fast directions between $27^{\circ}$ and $40^{\circ}$ for events shallower than $100 \mathrm{~km}$ observed by Audoine et al. (2004), but differs from a general NW alignment of $\phi$ observed on the temporary network CNIPSE (Styles, 2009). However, the deployment only covered a time span of six months (Styles, 2009) and could hence show deviations from the long term trend. In comparison to maximum horizontal compressive stress, $\phi$ trends slightly more north than estimates from focal mechanisms, i.e. $S_{H, \max }=67 \pm 11^{\circ}$ after Sherburn et al. (2009) and $\sim 58^{\circ}$ after Hurst et al. (2002). Station KATZ has a bimodal distribution, with one set of directions roughly perpendicular to $128 \pm 11^{\circ}$ (Darby et al., 2000) and the other set more or less parallel to an estimate for $S_{H, \max }$ of approximately $120 \pm 12^{\circ}$ (Sherburn et al., 2009). From the deep measurements, $\phi=39 \pm 2^{\circ}$ at station HATZ agrees with the trend expected after the study by Darby et al. (2000). This deep mean is also roughly trench parallel, similar to fast directions from $S K S$-splitting, which have 
been attributed to anisotropy from the mantle wedge by Greve et al. (2008). The fast directions at the rest of the stations where a relatively meaningful trend can be observed agree more with the stress estimates from focal mechanisms (Sherburn et al., 2009; Hurst et al., 2002) and local splitting studies (e.g. Styles, 2009; Greve et al., 2008, and references therein). In addition, stations ARAZ, KATZ, POIZ and WHTZ are in areas with a large number of active, NE striking faults. Stations KATZ and RATZ are in an area south of the accommodation zone where the local stress direction estimate from Sherburn et al. (2009) is more likely to be valid than the regional trend from Darby et al. (2000). There is only a slight backazimuth effect on fast directions at station KATZ (as discussed previously and seen in figure 8.3), so local sources of anisotropy must largely be responsible for the bimodal distribution of $\phi$. It could be interpreted as the result of a combination of stress-induced and structural anisotropy, where only slightly varying, individual ray paths sample either stress or structure: Faults are situated in direct vicinity to the station, and are covering a wide range of backazimuths. In combination with a macrofault spacing wider than the fresnel zones ${ }^{35}$ of the respective incoming waves a bimodal distribution of fast direction without backazimuth dependence could be caused. Similarly, the stations in areas of active faulting north of the lake could be influenced by structural anisotropy. However, because the strike of normal faults does not deviate significantly from maximum horizontal compressive stress estimates, we cannot determine the dominant mechanism causing the observed anisotropy in this area.

\subsubsection{Deformation}

Baseline changes and time series The only strong change in baseline lengths common to a large number of stations is the increase around 08-09/2007. This change could be due to extension caused by an inflating magma source, as modelled by Jolly et al. (2008). However, the signal in the time series intially suggested to be due to volcanic unrest at lake Taupo (Jolly et al., 2008) can be seen on stations relatively far away from the caldera, such as RGHR, for example (figure 8.4). It may be inferred, that the signal might not be related to volcanic unrest but rather to an overall tectonic trend or maybe even just to a systematic error in one of the processing steps.

Strain field The strain field was computed for the last six out of seven time periods (periods IV-VII are shown in figure 7.4). However, period II is not shown and interpreted because the combination of four available stations with the computation parameters only yields five significant cells. Furthermore, although period III with five stations available shows a higher number of significant cells, there is almost no variation in shortening axes direction. This is mainly due to two reasons: (a) With five stations and four nearest neigbours many grid cells will "see" the same defor-

\footnotetext{
$\overline{35}$ i.e. the volume "sampled" by the wave due to wavelength and amplitudes
} 


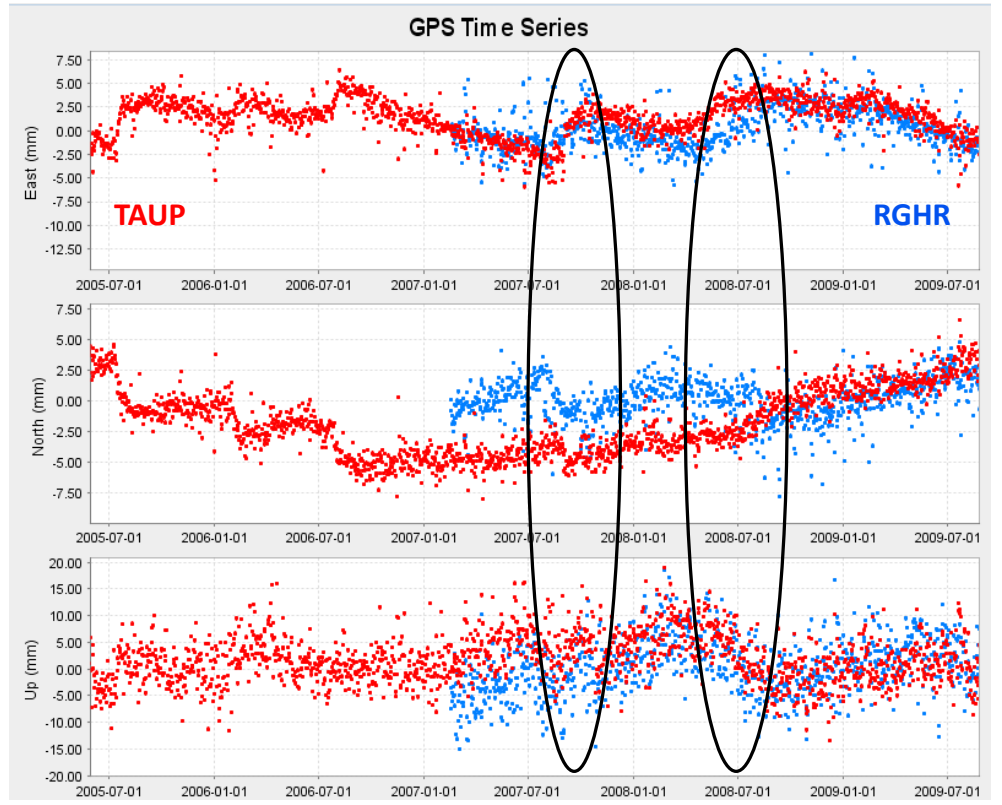

Figure 8.4:

Detrended

GPS timeseries

for stations

TAUP (red)

and RGHR

(blue). Both

show strong

changes in 2007

and 2008, al-

though RGHR

is further

away from the

caldera (see

figure 6.1 for

station loca-

tions). Data

obtained and

figure modified

from GeoNet.

mation; and (b) deformation in this relatively long period (almost one year) was relatively uniform at all stations and mostly did not show particularly big jumps. The resulting strain is hence most likely adequate to be compared to the regional tectonic regime: With the mean shortening axes over the well constrained periods IV-VII in direction of $42 \pm 10^{\circ}$ we find an agreement with the regional trend of extension (e.g. Beavan and Haines, 2001) perpendicular to our shortening axis. Darby et al. (2000) estimate extension at $128 \pm 11^{\circ}$, as expected approximately perpendicular to our estimate. Additionally, fault traces especially in the northern part of lake Taupo trend $45^{\circ}$ (Rowland and Sibson, 2001), i.e. parallel to our estimated extension direction.

For the four periods with a relatively good resolution, there is a variation between the time spans in the individual grid cells. However, no striking systematic pattern can be observed. While some cells again show shortening axes approximately parallel to the regional trend of rifting, there are deviations especially on the western side of an imaginary diagonal trending NE-SW (see strain fields in figure 7.9). These deviations from the regional trend could be due to different processes governing the so-called "accommodation zone" from the northern shore of the lake to the southern end of the caldera, a slightly more northerly trend of faults in the Northwest (Rowland and Sibson, 2001) and the influence of the Wairakei geothermal field (Darby et al., 2000). 


\subsubsection{Integrating GPS and shear-wave splitting}

Correlation of GPS time series with $\phi$ and $\delta t \quad$ Four GPS sensors are located in close proximity to seismometers. As seismometer WHTZ only has very few splitting data, we do not consider a comparison to deformation. For the other three seismometer-GPS pairs (HATZ-TGOH, RATZ-TGRA and WATZ-TGHR) deformation was plotted together with the moving means of $\delta t$ and $\phi$ as time series. While the east and north component showed no similarity to moving means of either fast direction or time delay, the vertical component takes a similar shape as the 30 point moving mean of $\phi$ (figure 8.5) at two stations. However, testing the data for correlation does not reveal a significant relation at any station. Furthermore, correlation of the GPS data with the mode instead of the mean at the same stations does not show significantly correlated components.

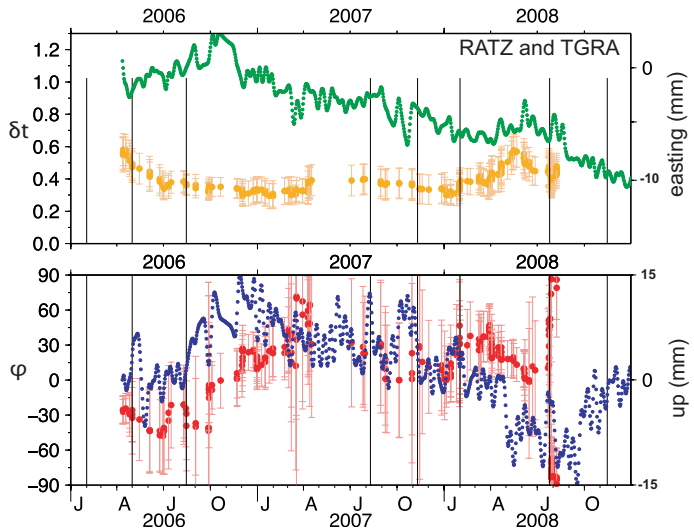

(a) RATZ - TGRA

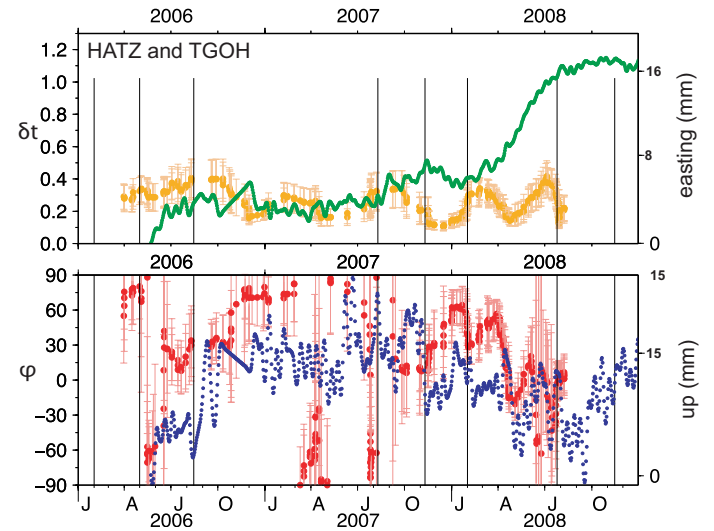

(b) HATZ - TGOH

Figure 8.5: Comparison of GPS timeseries and shear-wave splitting parameters at stations with locations close to each other around lake Taupo. Green and blue dots are easting and up, respectively. Orange and red dots are $\delta t$ and $\phi$ with $95 \%$ confidence error bars, respectively. Black vertical bars separate time periods.

Similarly, neither visual inspection of moving means and modes versus changes in baseline lengths nor computation of correlation coefficients suggest a direct relation between shear-wave splitting parameters and deformation.

Summary of findings at Taupo caldera Neither shear-wave splitting fast directions nor delay times show striking systematic changes in the time span investigated. Although GPS time series and baselines exhibit variations that could be related to an inflating magma chamber, this does not appear as a distinct feature in the strain field. This could be due to the poor station coverage in the caldera, i.e. the fact that the caldera is filled with water which makes direct observations difficult. In combination with the observation of a very similar signal at station RGHR however, we cannot exclude that the variation seen in the time series is a more regional tectonic 
feature instead of a local volcanic signature. Accordingly, if the same deformation signal is observed over a wide area, i.e. the area is behaving as a "block", relative positions of points within this block will most likely not undergo any changes, and hence we will not "see" this signal in shear-wave splitting. We infer that Taupo caldera during the investigated time span might not experience any significant volcanic unrest that can be extracted from shear-wave splitting or GPS data with relatively simple methods and the time constraint of this project. Further study is required to extract a possible volcanic deformation signal from the overall tectonic movement.

Similarly, the shear-wave splitting results indicate that the time variations might be only apparent temporal changes. A detailed analyses of earthquake source locations and particularly of waveforms is advised in order to extract similar events to investigate real temporal variations for future work in this area (G. Bokelmann, pers.comm., 2011), but beyond the scope and time frame of this study.

Despite these limitations, shear-wave splitting shows a good agreement with stress estimates from previous studies at certain times and stations. This is consistent with other studies from rift zones, where $\phi$ was found subparallel to the strike of the rift (e.g. Kyushu, Kaneshima et al., 1989, or Hawaii, Savage et al., 1989). Similarly, the mean $\phi=37 \pm 3^{\circ}$ is roughly parallel to the mean direction of shortening axes of $42 \pm 10^{\circ}$. In addition, some stations show $\phi$ subparallel to large fault systems, indicating potential structural structural anisotropy, as has been previously pointed out by e.g. Boness and Zoback (2006) and Johnson et al. (2011, in prep.). However, it remains unclear why for example in cases where estimated stress directions disagree with our strain directions we sometimes see shear-wave splitting fast direction aligned with stress, sometimes with strain and sometimes a bimodal distribution with both directions.

\subsection{Aso caldera}

\subsubsection{Influences on shear-wave splitting results}

Dependence of $\delta t$ and $\phi$ on earthquake parameters To exclude source and path effects as possible sources for the observed changes in fast direction, we checked the earthquake catalog for repeating earthquakes and/or earthquake families. Again, BCSEIS (Du et al., 2004) did not yield earthquake families with hypocentres in approximately the same location occurring over ideally the whole eight years. Instead, we visually determined earthquake clusters from plots of the catalog locations around Aso caldera. We then applied a simple catalog search for earthquakes with epicentres around the estimated centre of each cluster. The maximum allowed deviation in latitude and longitude from the centre was $0.04^{\circ}$ or approximately $4-5 \mathrm{~km}$. Furthermore, we constrained the hypocentres to depth shallower than $20 \mathrm{~km}$. The parameters of the seven clusters found are summarised in table 8.1 and their locations can be seen in figure 8.6. Although the contribution of each cluster to each of 


\begin{tabular}{|l||l|l|l|}
\hline cluster number & centre latitude $\left[^{\circ}\right]$ & centre longitude $\left[^{\circ}\right]$ & number of earthquakes \\
\hline \hline 1 & 33.033 & 131.0 & 43 \\
\hline 2 & 32.92 & 131.0 & 70 \\
\hline 3 & 32.76 & 130.85 & 30 \\
\hline 4 & 32.85 & 130.92 & 42 \\
\hline 5 & 32.88 & 130.68 & 12 \\
\hline 6 & 33.065 & 131.165 & 33 \\
\hline 7 & 33.117 & 131.18 & 34 \\
\hline
\end{tabular}

Table 8.1: Summary of characteristics of earthquake clusters found around Aso volcano.

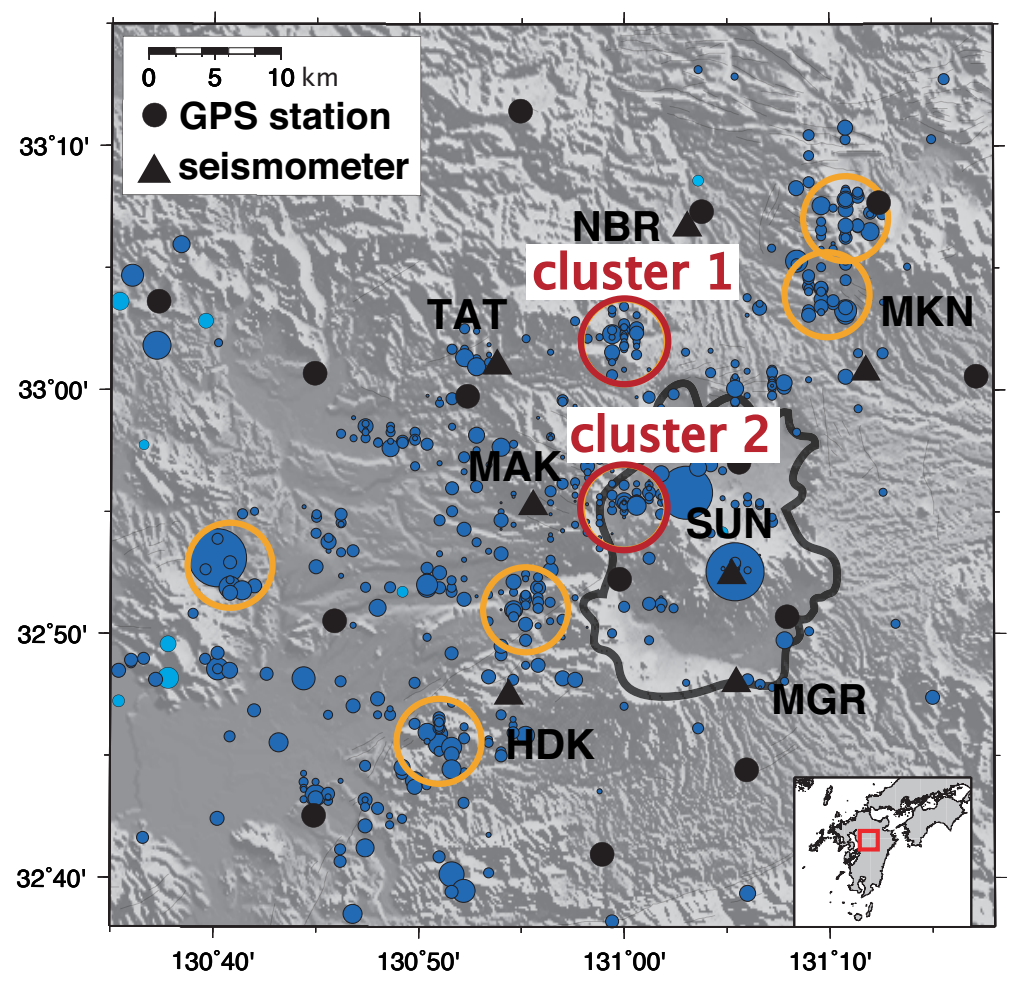

Figure 8.6: Location of earthquake clusters at Aso caldera: Red circles with labels are clusters 1 and 2 (see table 8.1 and figure 8.7), orange circles are all other clusters. 


\begin{tabular}{|l||l|l||l||l|l|}
\hline & minimum & maximum & & minimum & maximum \\
\hline \hline latitude $\left(^{\circ}\right)$ & 32.73 & 33.14 & longitude $\left(^{\circ}\right)$ & 130.65 & 131.21 \\
\hline distance $(\mathrm{km})$ & 3.29 & 51.09 & depth $(\mathrm{km})$ & -2.06 & 19.42 \\
\hline magnitude & 0.1 & 3.8 & $\begin{array}{l}\text { angle of inci- } \\
\text { dence }\left[^{\circ} \text { ] }\right.\end{array}$ & 5.8 & 39.3 \\
\hline$\delta \mathrm{t}(\mathrm{s})$ & 0.01 & 0.35 & error in $\delta \mathrm{t}(\mathrm{s})$ & 0.0 & 0.06 \\
\hline$\phi\left(^{\circ}\right)$ & -89 & 90 & error in $\phi\left(^{\circ}\right)$ & 1 & 24.25 \\
\hline
\end{tabular}

Table 8.2: Summary of parameters from shear-wave splitting at Aso volcano, for earthquakes in clusters. Depth in relation to sea level.

the time spans defined in section 6.2.2 varies, the use of the clusters is an improvement to just using all results with highly varying locations. It has to be stressed that despite the improved certainty in the nature of changes in shear-wave splitting parameters, the number of measurements is drastically reduced by this selection. Outliers are less likely to be identified and care has to be taken when time spans with a low number of measurements are analysed.

Splitting results and earthquake parameters from the events within the clusters are summarised in table 8.2. Note that due to a simpler velocity model used at Aso volcano than at lake Taupo ${ }^{36}$, we accept incidence angles of up to $40^{\circ}$ (M.Savage, 2010, pers.comm.). Figure 8.7 shows that the splitting results from only the clusters undergo the same variations as all results. An apparent temporal variation due to an actual change of earthquake sources can hence be excluded.

As for lake Taupo, we examined the dependence of several of the shear-wave splitting/earthquake parameter pairs. Examples are shown for stations TAT and MGR in figure 8.8. It can be seen that there are no major dependencies that could cause an apparent temporal variation.

Fast directions and structure $\phi$ at station MAK is parallel to faulting in this area (see figure 7.12). However, as this is also the direction of maximum horizontal compressive stress $\left(106^{\circ}\right.$, Townend and Zoback, 2006) we cannot determine whether observed anisotropy is due to stress or structure. Station TAT shows a bimodal distribution, one set subparallel to the regional stress and the other set oriented ENE. This second subset is dominant in periods III and V. As there is no major faulting in direct proximity to the station and since the different subsets seem to represent time periods, we infer that stresses exert the major control on splitting fast directions at this station. Similarly, stations NBR, MKN, MGR and SUN do not have major faulting in their direct vicinity, suggesting stress induced anisotropy as the main

\footnotetext{
${ }^{36}$ At lake Taupo our velocity model to calculate incidence angles contains a strong low velocity layer on top of the model, with the result that most incidence angles will be within the shearwave window. For Aso, we do not have enough constraints for such a model. However, since it is likely that a similar surface layer exists we extend the range of acceptable incidence angles slightly.
} 


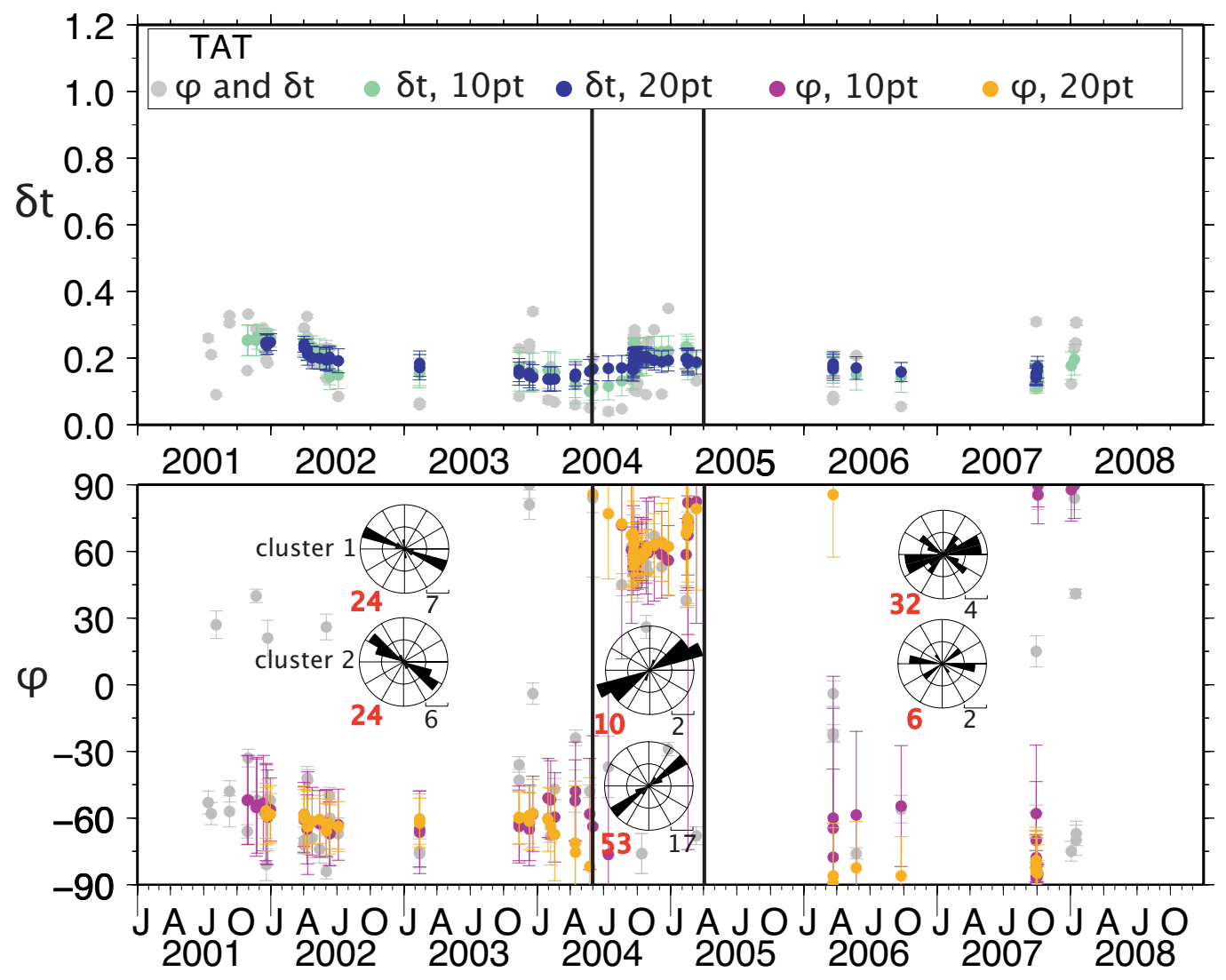

Figure 8.7: Moving means over results from clusters at station TAT show the same changes as results from all events at this station (figure 7.11). Green and blue dots are ten and 20 point moving means over $\delta t$, respectively. Purple and orange dots are ten and 20 point moving means over $\phi$, respectively. Black bars separate time periods for which the moving mean suggests a strong change. Insets show the rose diagrams at station TAT for those time periods for cluster 1 (upper row) and cluster 2 (lower row). Red numbers are number of measurements and black numbers are for scale of rose diagrams. For cluster locations refer to figure 8.6. Both clusters independently show the same change and we can hence exclude an apparent temporal change in splitting parameters due to changing earthquake source locations. 

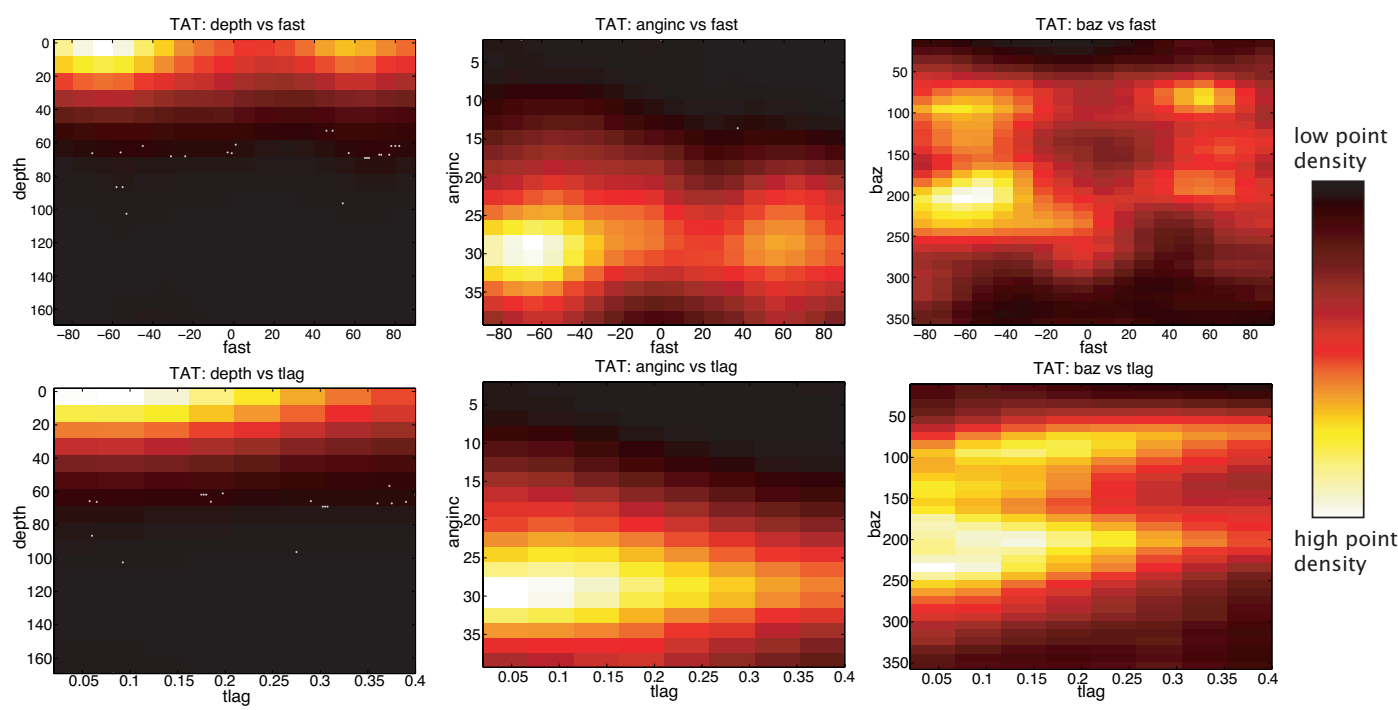

(a) TAT
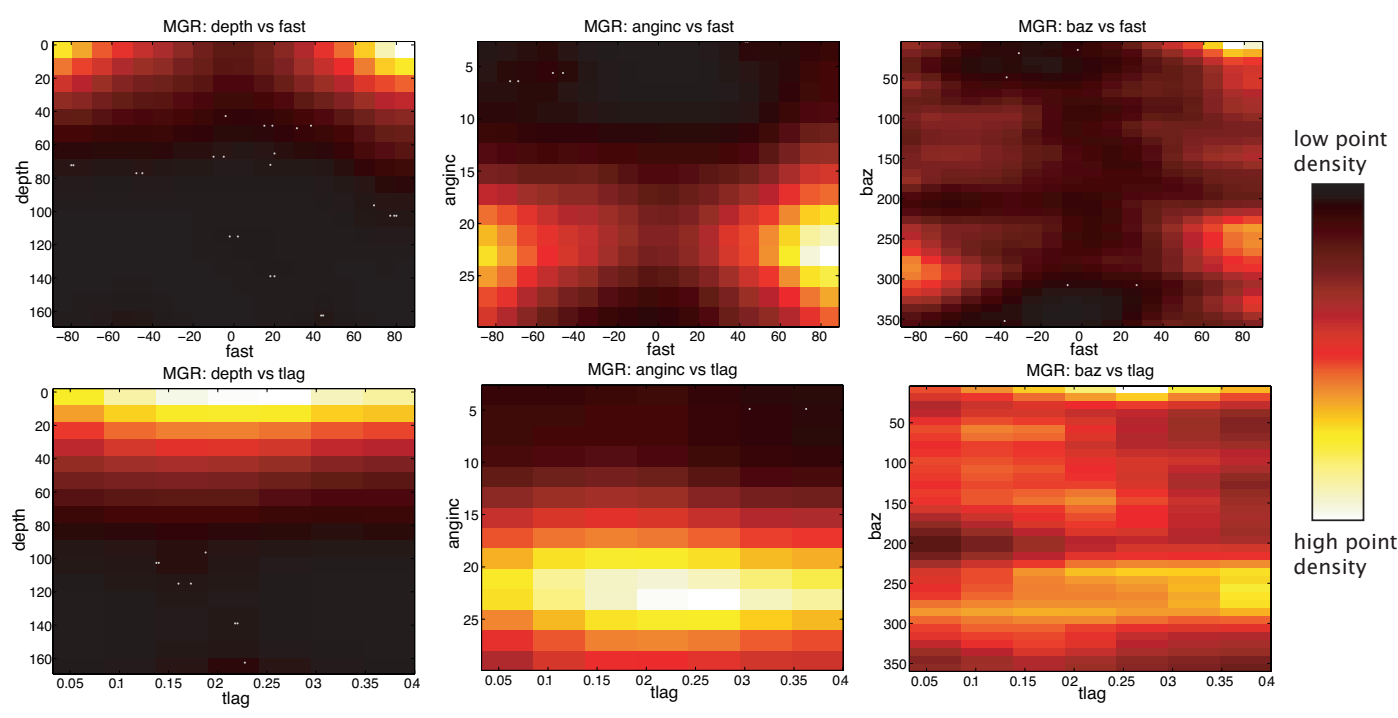

(b) MGR

Figure 8.8: Dependencies of $\phi$ and $\delta t$ on earthquake parameters at two stations at Aso caldera. Colours indicate point densities. The white dots that show up in a few plots are artifacts from the density calculation. The upper row shows is for $\phi\left(\right.$ fast, in $\left.{ }^{\circ}\right)$, the lower row for $\delta t$ (tlag, in seconds). The first column shows depth (in $\mathrm{km}$ ), the second angle of incidence (anginc, in ${ }^{\circ}$ ) and the third backazimuth $\left(\right.$ baz, in $\left.{ }^{\circ}\right)$. 
factor causing shear-wave splitting. The general trend at those stations is not parallel to the regional stress estimate for the caldera $\left(S_{H, \max }=106^{\circ}\right.$, Townend and Zoback, 2006), so a stress estimate of $S_{H, \max }=75^{\circ}$ from further south (Townend and Zoback, 2006) or local stress sources might be more important for those stations. Note that station NBR shows fast directions oriented roughly north-south. This confirms the findings by Kaneshima and Ando (1989) for an area north of Aso caldera. They conclude that the stress regime is complicated in this area and possibly influenced by en echelon transform faulting connecting offset parts of the rift zone in the northsouth direction. This might explain the NS alignment of $\phi$ at NBR. Station HDK has a major portion of fast directions trending WNW-ESE parallel to the regional stress mentioned above. However, a second, minor trend oriented parallel to ENE-WSW striking faults, dikes and veins (Kamata, 1989) can be observed especially for the deep subset of events. As mentioned above, the maximum horizontal compressive stress southwest of the caldera strikes $\mathrm{N} 75^{\circ} \mathrm{E}$ (Townend and Zoback, 2006), so again we cannot determine whether anisotropy is due to stress or structure at station HDK.

Crack density Delay times can be indicative of the amount of anisotropy along the ray path (see section 3.1). If anisotropy is related to cracks, shear-wave anisotropy is equal to crack density for $v_{P} / v_{S}=1.732$ (i.e. for a Poisson solid, after Crampin, 1994, and references therein). Budiansky and O'Connell (1976) define crack density $\epsilon$ based on elastic deformation energy in an otherwise uniform medium as

$$
\epsilon=\frac{N a^{3}}{V}
$$

where $N$ is the total number of cracks, $a$ is the average radius of the cracks and $V$ is the volume of the elastic solid. After Leary et al. (1990, and references therein) $\epsilon$ can be estimated from delay time and S-wave travel time through the anisotropic medium $t_{S}$ as

$$
\epsilon=\frac{\delta t}{t_{S}}
$$

The mean S-travel time for all stations and time periods is $14.7 \mathrm{~s}$, and the mean depth is $14 \mathrm{~km}$. We assume that most anisotropy is occurring at a depth of $\sim 5 \mathrm{~km}$ and shallower (see section below about stress modelling and figure 8.10). Thus we can do an order of magnitude calculation: Since only a third of the mean vertical distance sampled by most ray paths is actually anisotropic, we estimate that only a third of the mean travel time is spent in an anisotropic medium, hence $t_{S}=5 \mathrm{~s}$. With a mean delay time of approximately 0.2 s equation 18 yields $\epsilon=0.04$ or $S W A$ $=4 \%$. Crampin (1994) reports values of $S W A$ between $1.5 \%$ and $4.5 \%$, but even $10 \%$ and higher for some volcanic settings. In our case we are dealing with a lower Poisson's ratio than for a Poisson's solid, hence $S W A$ might not exactly equal $\epsilon$. The value still seems to be on a similar order of magnitude. 
Relation to $v_{P} / v_{S}$-ratio The average ratio of $v_{P}$ to $v_{S}$ of $\sim 1.65 \pm 0.09$ for 2001-2007 is lower than previously reported values: Sudo and Kong (2001) and Hirose et al. (2008) observe $v_{P} / v_{S}$-ratios above 1.7 for upper, mid and lower crust in southwestern Japan. Both of them average over time periods different from our study (1981-1989 and 1997-2005, respectively), so we cannot exclude that difference is attributed to different stages of the volcano in its eruptive cycle. Furthermore, Hirose et al. (2008) get their estimate of $1.79 \pm 0.09$ from earthquakes all over Kyushu, Shikoku, and southwestern and central Honshu within the oceanic crust. It is possible that our estimate has a strong influence from volcanic activity and is hence not comparable to large scale studies. To exclude an effect from erroneous picks we checked the arrival times of $P$ - and $S$-phases. Despite some arrivals that we would have picked slightly differently (see figure 8.9) there are no major abnormalities that could account for the observed low mean value. Also note the high standard deviation on our mean, which makes the difference to the estimate of 1.704 with a standard deviation of 0.0078 about the average by Sudo and Kong (2001) statistically insignificant.

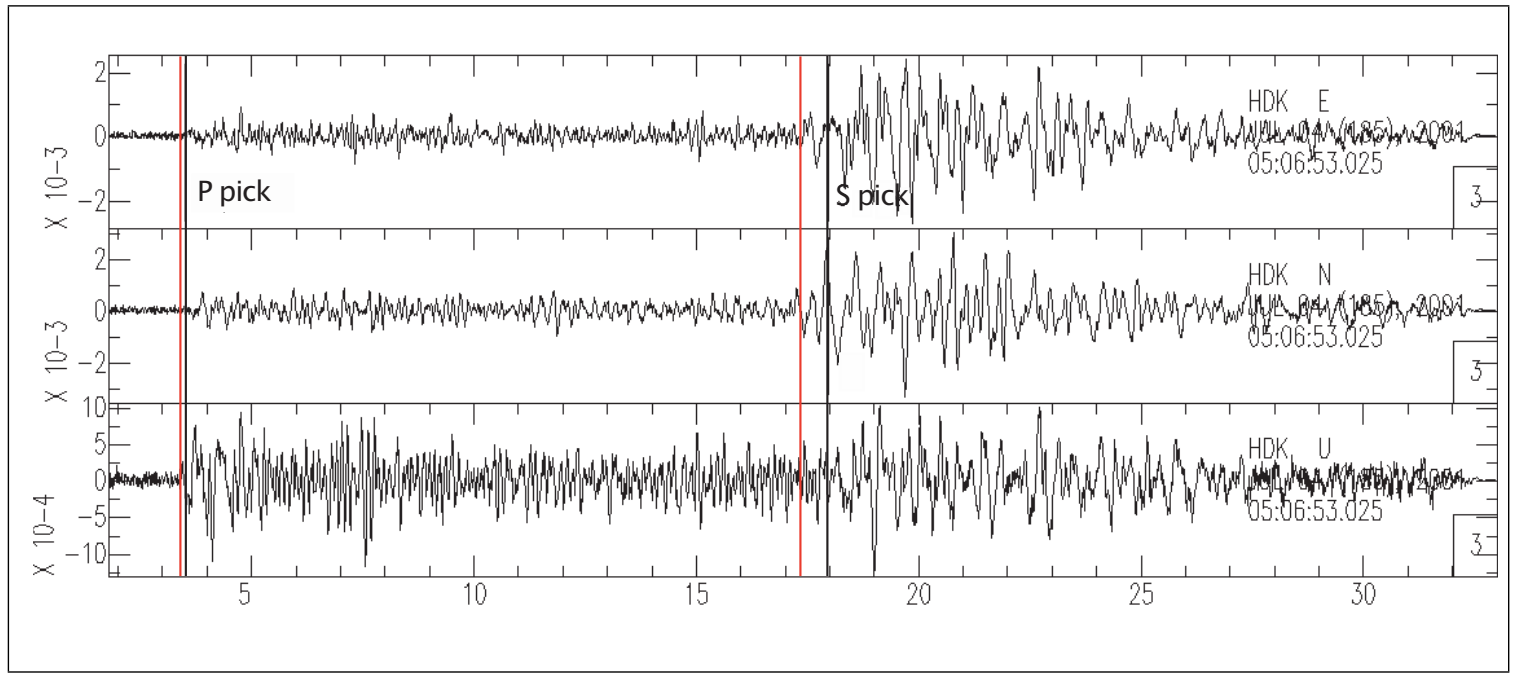

Figure 8.9: $P$ - and $S$-picks at station HDK for event 0107040506 . The black bars indicate the catalogue picks that were used for the splitting measurement, the red bar shows how both $P$ - and $S$-arrival could have been picked slightly differently. However, this minor shift cannot account for the low mean $v_{P} / v_{S}$-ratio observed throughout all years.

While the change in delay times at the northwestern stations in 2004 (see figure 7.11 and appendix D) by itself usually would most likely be considered as noise, the fact that a change in fast direction and in $v_{P} / v_{S}$-ratio occur during the same time period indicate a possible change not only in orientation of anisotropic features but also in crack density or amount of fluids, for example. There are complicated relationhips between $v_{P} / v_{S}$, pore pressure, amount and aspect ratio of cracks, presence and type of fluids etc (e.g. Nur and Simmons, 1969b; Anderson et al., 1974; Takei, 2002). Variations in $v_{P} / v_{S}$ in combination with seismic anisotropy, however, have been interpreted qualitatively to be related to the presence and/or movement 
of fluids in the crust (e.g. Lucente et al., 2010; Ozacar and Zandt, 2009).

The average $v_{P} / v_{S}$-ratios found here are not only lower than previous estimates in this area, but also among the lowest average values found in most settings. Nakajima et al. $(2001 \mathrm{a}, \mathrm{b})$ report an average $v_{P} / v_{S}$-ratio of 1.66 for the upper crust in an area around active volcanoes in northeastern Japan. Chatterjee et al. (1985) report an average value of $1.65 \pm 0.04$ for Yellowstone caldera and as low as $1.56 \pm 0.04$ for geothermal areas withing the caldera. Similarly, Vanorio et al. (2005) observe values down to 1.2 at Campi Flegrei and relate those values to gas enriched pore space. Higher values, but still relative lows in $v_{P} / v_{S}$-ratio around 1.7 have been observed at Campi Flegrei caldera as well as in the Taupo volcanic zone (Chiarabba and Moretti, 2006; Reyners et al., 2006). Kato et al. (2010) find an anomalous region with an average $v_{P} / v_{S}$-ratio of 1.66 above a potential magma intrusion coinciding with shallow crustal earthquakes.

Previous studies failed to explain such low $v_{P} / v_{S}$-ratios with different rock types and instead attribute the anomalous values to high crack aspect ratios $(\sim 0.1)$, potentially high crack densities (0.11-0.17), high pore pressures (i.e. low differential pressure between confining and pore pressure) and the presence of fluids or more likely gases (e.g. Tatham, 1982; Dvorkin et al., 1999; Takei, 2002; Vanorio et al., 2005; Lin and Shearer, 2009). Following the interpretations from Nakajima et al. (2001a) and Chiarabba and Moretti (2006) we suggest that the anomalously low $v_{P} / v_{S}$-ratios observed here result from the presence of gases in overpressurised, high porosity rock with high aspect ratio cracks $(\sim 0.1)$. Cooling and compacting magma from the eruption sequence in the early 1990s at Nakadake first crater (Ono et al., 1995) could be responsible for the release of gases and/or fluids and cause overpressured crack systems. Gas fluctuations have the potential to cause the observed variations in $v_{P} / v_{S}$ as well as changes in shear-wave splitting. If crack aspect ratio is high, it is possible that the cracks will not make the rock intrinsically anisotropic but act as heterogeneities instead, explaining why shear-wave splitting does not show anomalously high delay times and accordingly the obtained average crustal crack density of $\sim 0.04$ does not necessarily disagree with our argument. Abe et al. (2010) report a sill-like structure at $\sim 15 \mathrm{~km}$ depth that coincides with the top of deep low frequency earthquakes. Thus, similar to what has been suggested by Nakajima et al. (2001a) and Kato et al. (2010) who found low frequency earthquakes at the transition between high and low $v_{P} / v_{S}$ (i.e. potentially melt vs. gases), gas release from a potential magma reservoir at that depth might also be associated with the occurrence of this type of earthquake.

\subsubsection{Deformation}

As discussed in section 7.2.2, the GPS time series did not reveal particularly strong anomalies that could be correlated with our results. Baselines for different station pairs crossing the caldera reveal a lengthening in 2003 that opposes a general trend of shortening. This change is also seen in the $2 \mathrm{D}$ strain field, where the caldera stands 
out: Compressional areal dilatation dominates but changes to extension in 2003. This is in agreement with previous studies of GPS and levelling, where lengthening of baselines and inflation was detected (Geographical Survey Institute, 2004; Ohkura and Oikawa, 2008). No large earthquakes or slow slip events were reported that could account for the observed change. It is hence likely that this is resulting from a local, volcanic source, as opposed to the regional tectonics potentially causing baseline length variations at lake Taupo. While periods I, II, III and VI cover time spans on the order of a year or longer and have beginning and end approximately at the same time of year, strain for these times is most likely not affected by seasonal variations. Periods IV and V, however, only cover a few months each and are hence prone to errors due to seasonal effects. It is interesting that the change in strain precedes the variation in seismic anisotropy. Detailed analyses and interpretation will follow in the next section.

\subsubsection{Integrating GPS and shear-wave splitting}

Both shear-wave splitting fast directions as well as 2D strain shortening axes show variations in time and space. Crampin and Peacock (2005, and references therein) emphasise the role of small pore pressure changes in a crack-critical crust as a cause for $90^{\circ}$ flips in $\phi$, and such flips have been observed in association with eruptions at other volcanoes (e.g. Zaccarelli et al., 2009). The changes in fast direction observed here reach a maximum of $\sim 60^{\circ}$ and hence are most likely not related to the phenomen described above. As discussed in detail in sections 2.2 and 3.2, stress can influence shear-wave splitting and deformation. A relationship has been found previously in volcanic settings such as Mt. Asama, Japan, and Okmok volcano, Alaska (Savage et al., 2010b; Johnson et al., 2010). Accordingly, we model stresses around Aso caldera and compare them to our observations.

Coulomb modelling The software Coulomb 3.1 (Lin and Stein, 2004; Toda et al., 2005 ) was used to model stresses caused by different source models ${ }^{37}$ (figures 8.11 and 8.12). Furthermore, variations in the direction of maximum horizontal stress were computed based on Lund and Townend (2007) with codes by S.Greve (M.Savage, 2010 , pers.comm.), as well as the fit of $\mathrm{S}_{H, \max }$ with strain shortening axes and $\phi$.

With the stress tensor from Townend and Zoback (2006), equations from Zoback and Townend (2001) and parameter estimates from Brace and Kohlstedt (1980) we use an estimate for the regional stress as listed in table 8.3 and shown in figures 8.11 and 8.12. The calculations for regional stresses appear in appendix E.

Sudo and Kong (2001) analysed seismic velocities in the region around Aso and inferred the presence of a magma chamber at a depth of $\sim 6 \mathrm{~km}$ below the surface, underneath the central cones in the caldera, with velocities up to $31 \%$ lower than the

${ }^{37}$ Note that Coulomb 3.1 uses bar as input instead of the SI-unit GPa. For easy comparison with other Coulomb modelling we also use bar, but $G P a$ values will be given in parentheses. 
general seismic wave speeds in this area. Active source seismic experiments point towards the existence of hot, possibly molten rock at approximately the same depth and location (Tsutsui and Sudo, 2004) and the area has also been found earlier as location of high attenuation (Sudo, 1991). We use the magma chamber located $\sim 4 \mathrm{~km}$ west, $\sim 1.5 \mathrm{~km}$ south of Nakadake crater (Sudo et al., 2006) and at a depth of $5.8 \mathrm{~km}$ below sea level as a possible source for stress changes. In addition, Abe et al. (2010) use receiver functions to confirm the presence of a sill like structure at $\sim 15 \mathrm{~km}$ depth (coinciding with the top of a swarm of deep low frequency earthquakes) that has been suggested previously as source of deformation in 2003 (Ohkura and Oikawa, 2008). This deep source with square side lengths ${ }^{38}$ of $10 \mathrm{~km}$, located northeast of Nakadake first crater (Abe et al., 2010) is also incorporated into stress modelling. For the sill we tested tensile opening/closing of $0.01 \mathrm{~m}, 0.1 \mathrm{~m}, 1 \mathrm{~m}, 10 \mathrm{~m}$ and $100 \mathrm{~m}$, where $10 \mathrm{~m}$ turned out to be the smallest value that produced changes in $S_{H, \max }$ close to the locations of our stations. Assuming a comparable overall volume change in the Mogi source leads to a value of $1 * 10^{9} \mathrm{~m}^{3}$.

From the low $v_{P} / v_{S}$-ratio and the resulting low Poisson's ratio $\sigma$ it can be inferred that the elastic moduli in the area differ from a global average. We use an estimate of $v_{P}=4200 \mathrm{~m} / \mathrm{s}$, which is at the low end of the velocity spectrum found in this area (Sudo and Kong, 2001) and which has been used in other volcanic areas (e.g. Mordret et al., 2010), to calculate shear modulus $\mu=1.68 * 10^{5}$ bar or $16.8 \mathrm{GPa}$ (equations 19, 20, and an average density $\rho=2600 \mathrm{~kg} / \mathrm{m}^{3}$, Okubo and Shibuya, 1993 ) and derive Young's modulus $E=4.07 * 10^{5}$ bar or $40.7 \mathrm{GPa}$ (equation 21 ).

$$
\begin{gathered}
\frac{v_{P}}{v_{S}}=1.65 \\
v_{S}=\sqrt{\frac{\mu}{\rho}} \\
E=2 \mu(1+\sigma)
\end{gathered}
$$

Note that these values will most likely be lower limits due to the low velocity estimates. The volume change $\Delta V$ of the stress source is linked to shear modulus, source radius $a$ and overpressure $\Delta P$ via equation 22 (McTigue, 1987).

$$
\Delta V=\frac{\Delta P a^{3} \pi}{\mu}
$$

If $\mu$ increases, a higher overpressure and/or source radius are required to cause the same volume change. However, a higher estimate of $E$, which is defined as the ratio

${ }^{38}$ Other studies use $5 \times 5 \mathrm{~km}^{2}$, but to get an idea of the concept we use twice the previously suggested side length, as a small source has an even lower effect on changing the directions from the regional stress field. A smaller sill might be modelled at a later stage. 
of tensional stress to the resulting extensional strain (Stein and Wysession, 2003) will require an even higher volume change to cause the same strain. This means that the modelled source sizes or overpressure could be required to be considerably higher to cause the same strain for a different set of elastic moduli.

As mentioned above, we calculate the fit of each model, that is the difference between the modelled $S_{H, \max }$, the observation-based strain shortening axes and $\phi$ : The average cosine of residual angles is computed with the L1-norm. A value close to 1 indicates average residual of 0 or $180^{\circ}$, i.e. a good fit ${ }^{39}$. The fit of stress and strain as well as stress and shear-wave splitting fast direction is then averaged to determine the best model for both data types. We compare $S_{H, \max }$ directions at $1 \mathrm{~km}$ depth to strain shortening axes. Although strain is here computed from surface measurements and should hence fit stresses closer to the surface, it should be influenced by the regional stress. Due to the stress values of 0 at the surface, however, we need to look at stress directions below the surface to "see" the regional stress. Shear-wave splitting fast directions are assumed to be representative for anisotropy along the whole ray path. Here, we chose $\phi$ at roughly $5 \mathrm{~km}$ to be compared to stresses at the same depth, which is a depth that $\sim 70 \%$ of all measurements sample (see figure 8.10). First of all we determine the importance of the regional stress: Models with just the regional stress direction give a good fit for both $\phi$ and strain. The values for the different periods are listed in table 8.4.

Furthermore we tested the fit of different source models with regional stress fields with high and low regional differential stress (see description above and appendix E) compared to the strain field and fast directions from shear-wave splitting. The models with low differential regional stress gave the best fit, although the differences in fit particularly between similar models were sometimes only on the third decimal ${ }^{40}$. $S_{H, \text { max }}$ directions will flip by $90^{\circ}$ for sources changing from deflation to inflation or vice versa in the direct vicinity of the source or if there is no or only minor influence from the regional stress field. Accordingly by comparing the observed fast directions and strain shortening axes to $S_{H, \max }$ we can be relatively certain about whether we have an inflating or a deflating source, i.e. about the general trend of stresses. In contrast, the uncertainty in distinguishing between a deflating sill and a deflating sill plus a deflating Mogi source is higher. In general, when looking at the stress-models from the different sources (including the regional stress) the differences in fit are not very large and are dominated by the fit of stress to strain. From strain calculations, however, we have additional information: While the fit is only calculated based on directions, we can test whether a model can reasonably explain the observed strain by looking at modelled vs. observed compressional or extensional dilatation.

Table 8.5 lists the best fitting models for the different periods, strain, splitting

\footnotetext{
${ }^{39}$ Note the nonlinearity of the fit values due to the cosine function. Further modelling will use a linear scale instead.

${ }^{40}$ i.e. a difference at a resolution that the splitting measurements do not have

${ }^{41}$ Best fitting model for this period has fit of 0.814 , but cannot explain observed strain field. Hence the second best fit was chosen as the best model.
} 


\begin{tabular}{|c|c|c|c|c|c|}
\hline \multicolumn{2}{|c|}{ regional stress } & $\begin{array}{l}\text { azimuth } \\
\left({ }^{\circ}\right)\end{array}$ & plunge $\left(^{\circ}\right)$ & surface value & $\begin{array}{l}\text { vertical } \\
\text { gradient } \\
\text { in bar } / \mathrm{km} \\
(\mathrm{MPa} / \mathrm{km})\end{array}$ \\
\hline $\begin{array}{l}\text { high diff. } \\
\text { stress }\end{array}$ & $\begin{array}{l}\text { S1 } \\
\text { S2 } \\
\text { S3 }\end{array}$ & $\begin{array}{l}102 \\
290 \\
198\end{array}$ & $\begin{array}{l}55 \\
35 \\
4\end{array}$ & $\begin{array}{l}0 \\
0 \\
0\end{array}$ & $\begin{array}{l}255(25.5) \\
196(19.6) \\
137(13.7)\end{array}$ \\
\hline $\begin{array}{l}\text { low diff. } \\
\text { stress }\end{array}$ & $\begin{array}{l}\text { S1 } \\
\text { S2 } \\
\text { S3 }\end{array}$ & $\begin{array}{l}102 \\
290 \\
198\end{array}$ & $\begin{array}{l}55 \\
35 \\
4\end{array}$ & $\begin{array}{l}0 \\
0 \\
0\end{array}$ & $\begin{array}{l}255(25.5) \\
245(24.5) \\
235(23.5)\end{array}$ \\
\hline $\begin{array}{l}\text { model } \\
\text { name }\end{array}$ & local source & $\begin{array}{l}\text { tensile } \\
\text { open- } \\
\text { ing/volume } \\
\text { change }\end{array}$ & $\operatorname{dip}\left(^{\circ}\right)$ & top $(\mathrm{km})$ & $\begin{array}{l}\text { bottom } \\
(\mathrm{km})\end{array}$ \\
\hline$\overline{\mathrm{A}}$ & $\begin{array}{l}\text { deflating } \\
\text { horiz. dike }\end{array}$ & $-10 \mathrm{~m}$ & 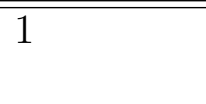 & 15 & 15.2 \\
\hline $\mathrm{B}$ & $\begin{array}{l}\text { inflating horiz. } \\
\text { dike }\end{array}$ & $+10 \mathrm{~m}$ & 1 & 15 & 15.2 \\
\hline $\mathrm{C}$ & $\begin{array}{l}\text { deflating mogi } \\
\text { source }\end{array}$ & $-1^{*} 10^{9} \mathrm{~m}^{3}$ & 90 & 5 & 6.6 \\
\hline $\mathrm{D}$ & $\begin{array}{l}\text { inflating mogi } \\
\text { source }\end{array}$ & $+1^{*} 10^{9} \mathrm{~m}^{3}$ & 90 & 5 & 6.6 \\
\hline $\mathrm{E}$ & $\begin{array}{l}\text { inflating horiz. } \\
\text { dike } \\
\text { inflating mogi } \\
\text { source }\end{array}$ & $\begin{array}{l}+10 \mathrm{~m} \\
+1^{*} 10^{9} \mathrm{~m}^{3}\end{array}$ & $\begin{array}{l}1 \\
90\end{array}$ & $\begin{array}{l}15 \\
5\end{array}$ & $\begin{array}{l}15.2 \\
6.6\end{array}$ \\
\hline $\mathrm{F}$ & $\begin{array}{l}\text { deflating } \\
\text { horiz. dike } \\
\text { deflating mogi } \\
\text { source }\end{array}$ & $\begin{array}{l}-10 \mathrm{~m} \\
-1^{*} 10^{9} \mathrm{~m}^{3}\end{array}$ & $\begin{array}{l}1 \\
90\end{array}$ & $\begin{array}{l}15 \\
5\end{array}$ & $\begin{array}{l}15.2 \\
6.6\end{array}$ \\
\hline G & $\begin{array}{l}\text { deflating } \\
\text { horiz. dike } \\
\text { inflating } \\
\text { magma cham- } \\
\text { ber }\end{array}$ & $\begin{array}{l}-10 \mathrm{~m} \\
+1^{*} 10^{9} \mathrm{~m}^{3}\end{array}$ & $\begin{array}{l}1 \\
90\end{array}$ & $\begin{array}{l}15 \\
5\end{array}$ & $\begin{array}{l}15.2 \\
6.6\end{array}$ \\
\hline $\mathrm{H}$ & $\begin{array}{l}\text { inflating horiz. } \\
\text { dike } \\
\text { deflating } \\
\text { magma cham- } \\
\text { ber }\end{array}$ & $\begin{array}{l}+10 \mathrm{~m} \\
-1^{*} 10^{9} \mathrm{~m}^{3}\end{array}$ & $\begin{array}{l}1 \\
90\end{array}$ & $\begin{array}{l}15 \\
5\end{array}$ & $\begin{array}{l}15.2 \\
6.6\end{array}$ \\
\hline
\end{tabular}

Table 8.3: Parameters for different stress models. Sill has to be modelled as a dike with almost no dip. Magma chamber is modelled as a Mogi source at the centre of a vertical (imaginary) dike. All models have a Poisson's ratio of 0.21 , a Young's modulus of 407,000 bar (40.7 GPa, see main body of text) and a coefficient of friction of 0.8 . 


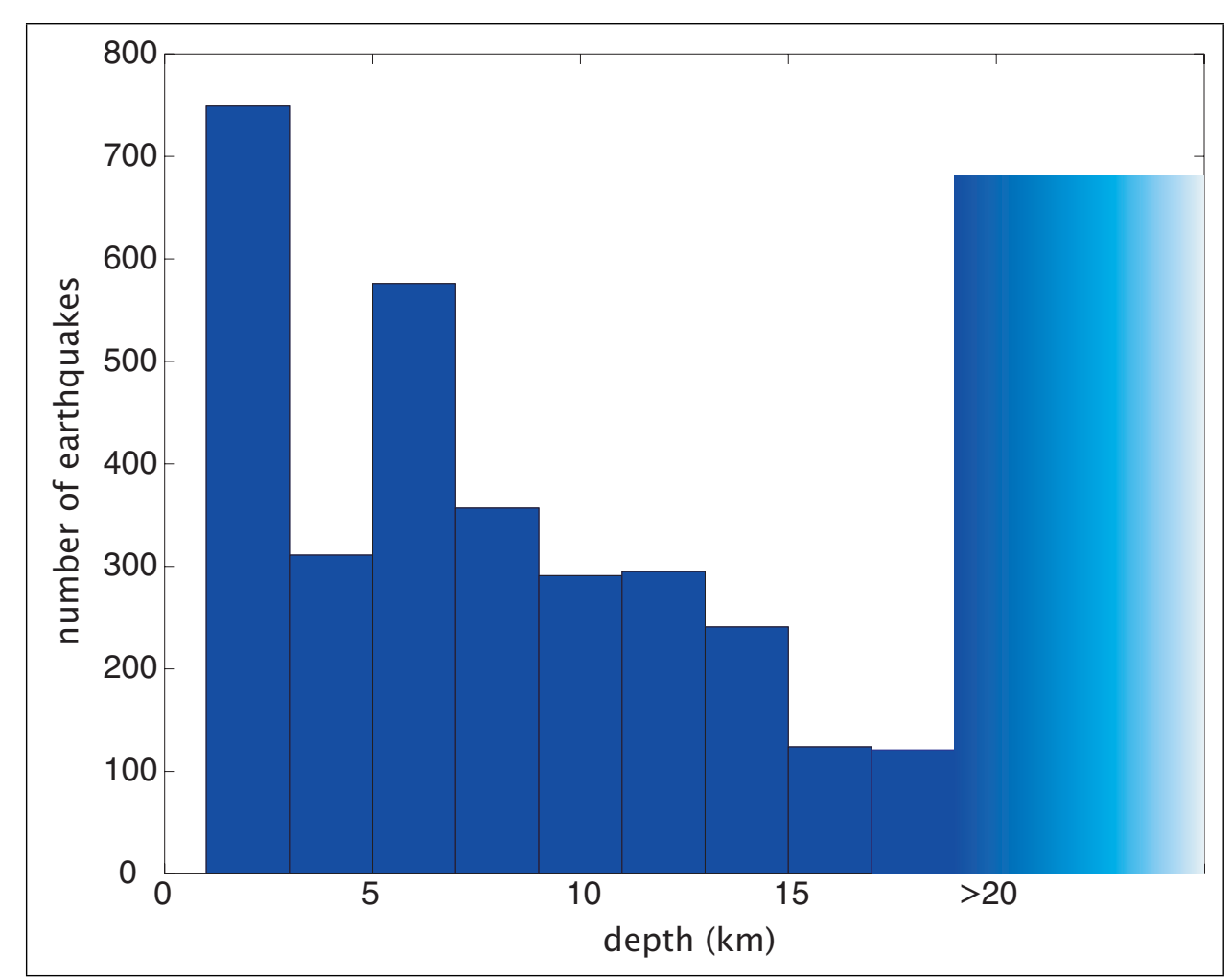

Figure 8.10: Depth distribution of earthquakes with splitting measurements for Aso caldera. Note that all events deeper than $20 \mathrm{~km}$ are placed in the bin on the far right.

\begin{tabular}{|l||l|l|l|}
\hline period & stress to strain & stress to splitting & strain to splitting \\
\hline \hline I & 0.952 & 0.699 & 0.648 \\
\hline II & 0.794 & 0.740 & 0.770 \\
\hline III & 0.919 & 0.739 & 0.706 \\
\hline IV & 0.800 & 0.738 & 0.715 \\
\hline V & 0.761 & 0.706 & 0.551 \\
\hline VI & 0.955 & 0.727 & 0.626 \\
\hline
\end{tabular}

Table 8.4: Fit of a model with just the regional stress $S_{H, \max }=106^{\circ}$ after Townend and Zoback (2006) compared to splitting and strain. Numbers are cosines of average residual angles between the different sets of directions: A value close to 1 hence suggests a good fit, the closer the values get 0 the worse the fit (i.e. the higher the average residual angle). 


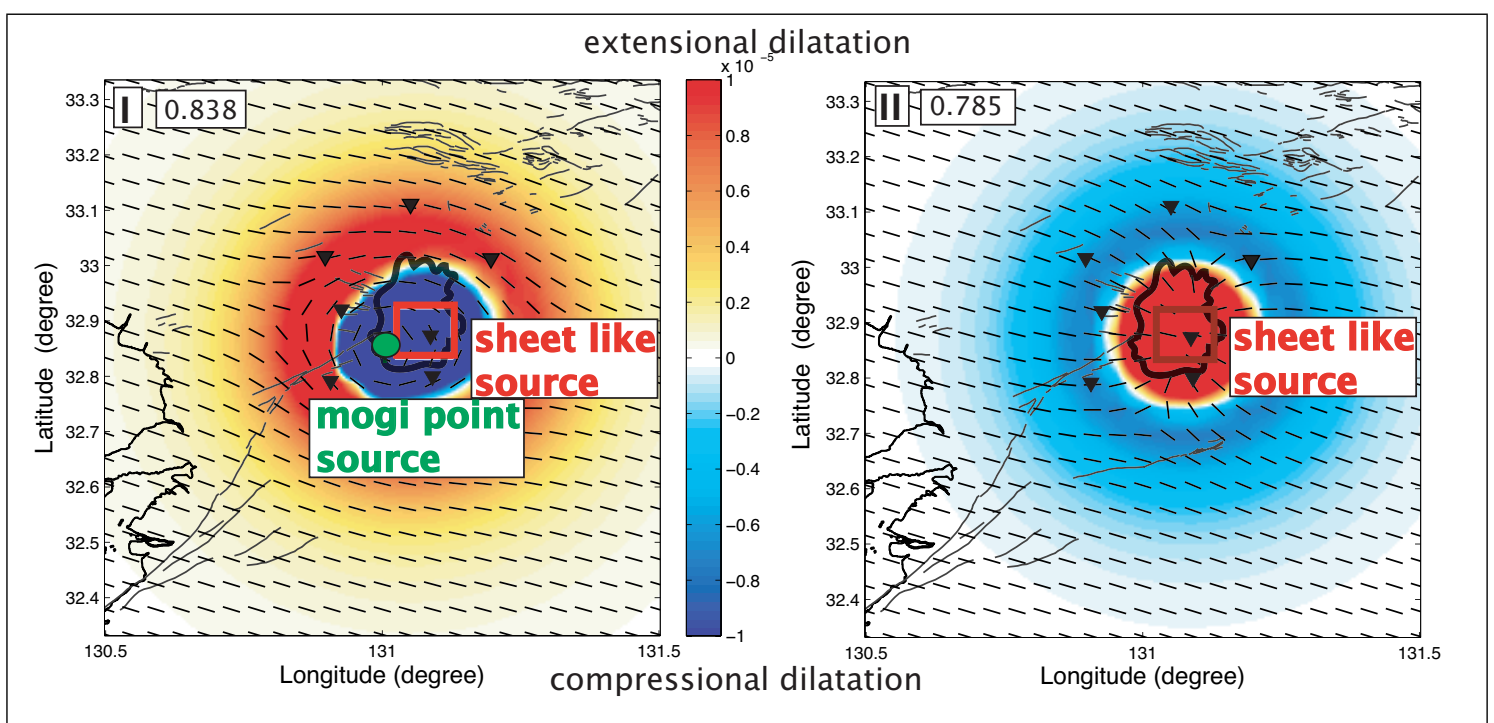

(a) Periods I-II

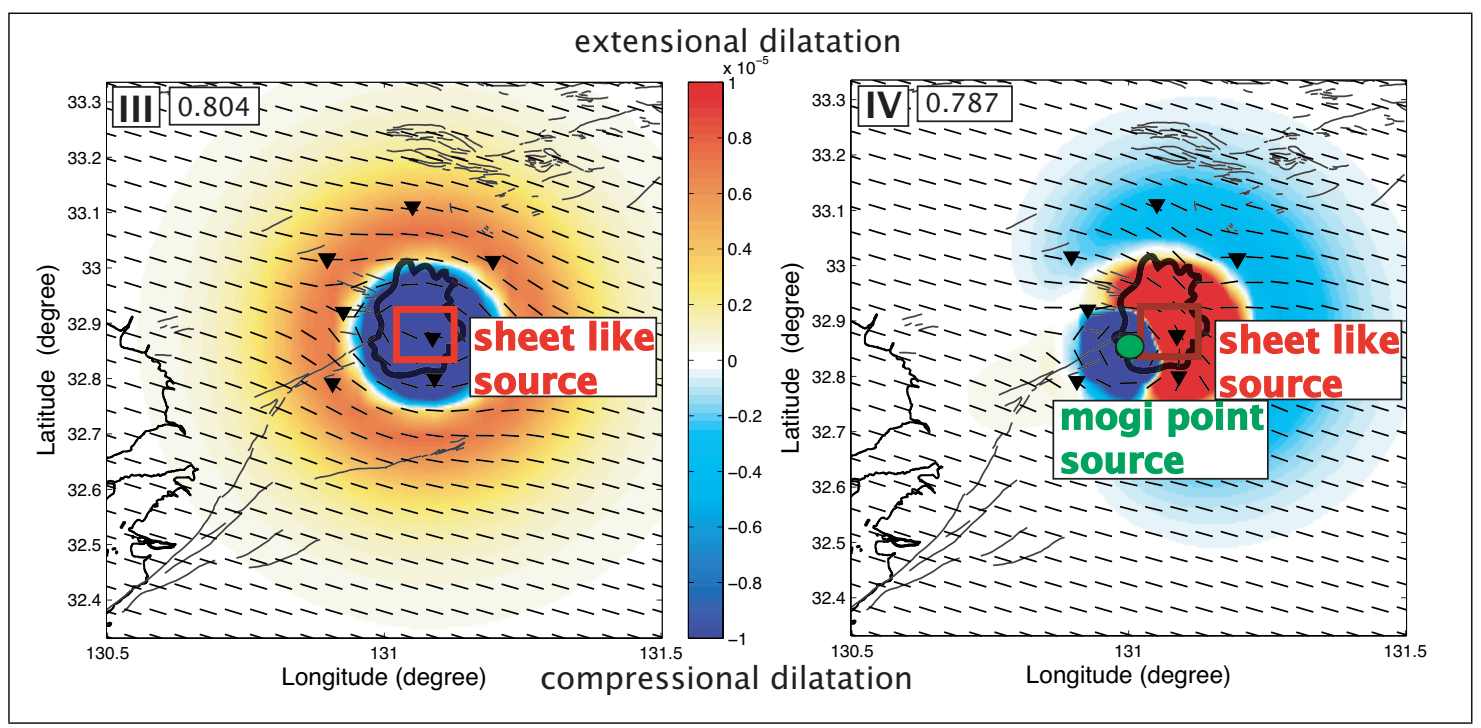

(b) Periods III-IV

Figure 8.11: Best fitting stress models at Aso caldera, periods I-IV. Colours indicate theoretical dilatation due to the modelled sources, black upside down triangles are seismometer locations for reference. Caldera boundary outlined in black. Black bars are $S_{H, \max }$ orientations, calculated for $1 \mathrm{~km}$ depth (since strain is fitted at $1 \mathrm{~km}$ and is dominating fit). Roman numbers in the upper left corners are period for which respective model is the best fit, with corresponding fit value. Compare $S_{H, \max }$ orientations to strain shortening axes in figure 7.14 and shear-wave splitting fast directions in figure 7.12. 


\begin{tabular}{|l||l|l|l|l|}
\hline period & model & overall fit & fit to strain & fit to splitting \\
\hline \hline I & F & 0.838 & 0.805 & 0.871 \\
\hline II & B & 0.785 & 0.829 & 0.740 \\
\hline III & $\mathrm{A}^{41}$ & 0.804 & 0.870 & 0.738 \\
\hline IV & H & 0.787 & 0.830 & 0.744 \\
\hline V & H & 0.745 & 0.778 & 0.712 \\
\hline VI & G & 0.824 & 0.929 & 0.719 \\
\hline
\end{tabular}

Table 8.5: Best fitting stress models for the different periods at Aso caldera. Letters A, B, F, G, H for different models refer to parameters listed in table 8.3.

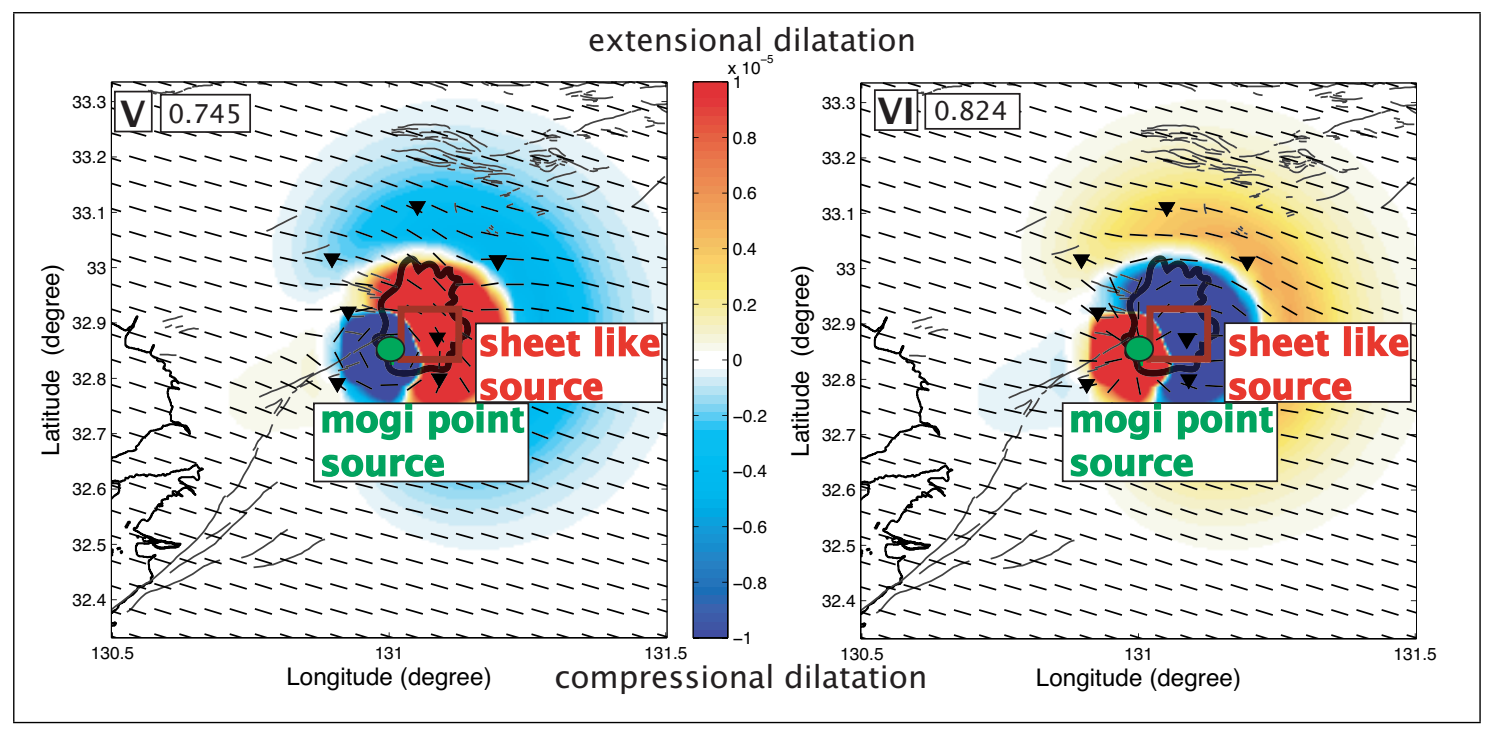

Figure 8.12: Best fitting stress models at Aso caldera, periods V-VI. Colours indicate theoretical dilatation due to the modelled sources, black upside down triangles are seismometer locations for reference. Caldera boundary outlined in black. Black bars are $S_{H, \max }$ orientations, calculated for $1 \mathrm{~km}$ depth (since strain is fitted at $1 \mathrm{~km}$ and is dominating fit). Roman numbers in the upper left corners are period for which respective model is the best fit, with corresponding fit value. Compare $S_{H, \max }$ orientations to strain shortening axes in figure 7.14 and shear-wave splitting fast directions in figure 7.12. 
and overall, and figures 8.11 and 8.12 show the corresponding directions and strain values. The best fit does not only fit directions but also matches nicely the observed dilatation (compare to figure 7.14) within the caldera for all time spans except period III. For this time span we chose the second best fit as the best model, since the difference between best and second best fit is only 0.01 (as opposed to a difference of up to 0.06 in comparison to other models) and the best fit fails to explain the observed strain field. In addition, the fits of the best models for all time periods are equal to or better than fits of stress models to shear-wave splitting data in a study on Asama volcano, where connections of seismic anisotropy to GPS were investigated: Savage et al. (2010b) report misfits of the L1-norm between roughly $50^{\circ}$ and $45^{\circ}$, which corresponds to fits of 0.643 up to 0.707 .

Stress models in the big picture We tested eight models with one or two sources (one flat sill-like source and one Mogi point source) with inflation vs. deflation in different combinations based upon initial, visual inspection of the strain field and findings from previous studies. We infer that the observed general trend of shortening of baselines and compression in the caldera is due to at least one deflating source. The best fit was achieved for a model of a deflating horizontal dike at $\sim 15 \mathrm{~km}$ depth and a deflating Mogi point source at $\sim 6 \mathrm{~km}$ for period I and only the deflating dike for period III. According to our model, the observed extensional areal dilatation in 2003 (period II) can be explained by inflation of the sill with a vertical tensile opening on the order ${ }^{42}$ of $10 \mathrm{~m}$. This value is higher than an opening of $70 \mathrm{~cm}$ suggested previously (Abe et al., 2010, and references therein). Periods IV and V (Nov 2004 - Mar 2005) are shorter and hence probably contaminated by seasonal deformation signals, the best fitting models indicate inflation of the horizontal dike and deflation of the Mogi source. This is in agreement with volcanic activity reported at Nakadake first crater (Aso Volcanological Laboratory, 2007), shown in figure 8.13 and summarised below:

- increasing seismicity since 2002

- increasing crater wall temperature up to $500^{\circ} \mathrm{C}$ from $2002-2003$, followed by decrease until mid 2005

- constantly high crater lake temperatures between $60-80^{\circ} \mathrm{C}$ in $2003-2005$

- crater lake level episodically decreasing in 2003-2006; totally dry crater in 2005

Ash emission events occurred at the active Nakadake first crater in July 2003, January 2004 and throughout April - July 2005 (see figure 8.13) and have been suggested to be due to the rise of newly ascending magma (Miyabuchi et al., 2008). Our models support this theory: Inflation of the sill in 2003 and rise of magma to even shallower levels could have caused the observed phreatomagmatic eruptions

\footnotetext{
${ }^{42}$ Note that all our stress models are trends and order of magnitude calculations.
} 


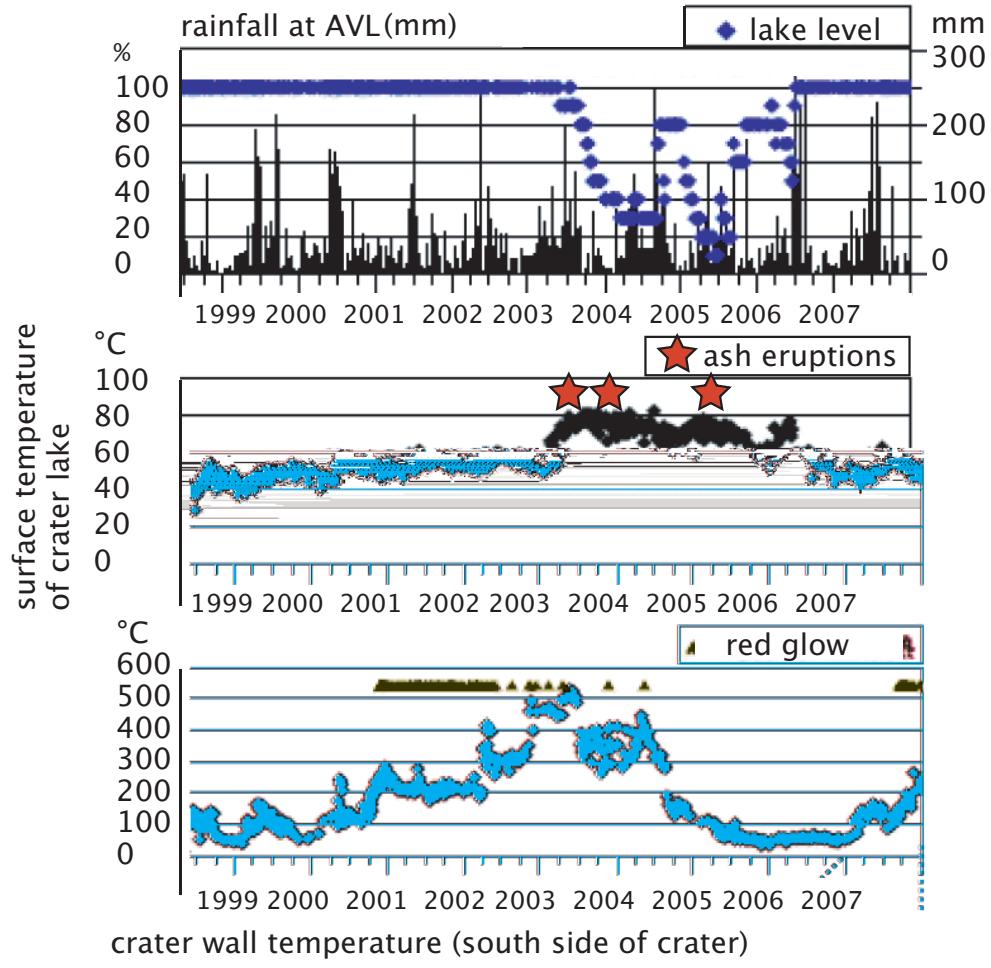

Figure 8.13: Monitoring parameters from Nakadake first crater. Lake level (blue diamonds) shows no strong relation to rainfall. Occurrence of red glow (red triangles) on the crater wall, high temperatures in the crater lake and on the crater wall, and ash emission (red stars) mark a period of higher than usual activity building up from 2001 onwards. Figure modified from Aso Volcanological Laboratory (2007).

(Miyabuchi et al., 2008; Aso Volcanological Laboratory, 2007). Eruptions stopped at the time when our models suggest a pause in magma input, probably accompanied by depressurisation and resulting deflation. Our models indicate possibly a new pulse of magma from deep levels from the end of 2004 until early 2005 (periods IV and V), which could have triggered a series of phreatomagmatic eruptions in 2005. Deflation in the Mogi source might be due to transport of magma to shallower levels and ongoing depressurisation. The average strain and splitting directions for those periods, however, are less well constrained. The eruption sequence is then followed by a calm period in 2006-2007 (Aso Volcanological Laboratory, 2007), for which our stress model suggests deflation of the sill but inflation of the magma chamber that could represent the preparation of a new period of eruptions. The pressure needed for inflation periods could be due to ascending magma. However, it is more likely that only a relatively small amount of magma intruded into the deep source and caused some migration upward leading to the eruption sequences, since the presence of a large amount of melt would give an increase in $v_{P} / v_{s}$-ratio instead of the low values observed here (e.g. Nakajima et al., 2001a,b; Chiarabba and Moretti, 2006). Instead, locally varying overpressure in the host rock could be created due to the release of gases from existing (and possibly newly intruded) magma triggered by the small scale intrusion, explaining the different magnitudes and slightly different timing of variations in shear-wave splitting at the stations in the region around the caldera. The presence of gases in overpressured cracks can also account for the consistenly low $v_{P} / v_{S}$-ratios observed at all stations. 
Limitations Although the models fit our data and agree well with other observations at Aso volcano, we have to keep in mind limitations:

- Coulomb 3.1 cannot model topography, which has been shown to have a significant influence on deformation (e.g. Cayol and Cornet, 1998) and to have the potential to change stress directions and magnitudes (e.g. Liu and Zoback, 1992; Miller and Dunne, 1996; Martín-Velázquez et al., 2009). Especially at Aso caldera the relief is strong: The central cones have an elevation of over $1 \mathrm{~km}$ above sea level, similar to the caldera rim in some parts, whereas the caldera floor is only at $\sim 500 \mathrm{~m}$ a.s.l. and the outer slopes of the caldera go down to 100-200 $\mathrm{m}$ or less within a few kilometers. Furthermore, we only model elastic processes. Incorporating visco- or poroelastic effects could also have a significant impact on stress directions and the corresponding fit.

- Our models are very simplistic and do not incorporate sources unrelated to the caldera or shallow processes in the caldera. A crack-like conduit detected by Yamamoto et al. (1999) at $300 \mathrm{~m}$ beneath the central cones and processes at the locations of earthquake swarms above the conduit (Mori et al., 2008) are unlikely to influence shear-wave splitting at most stations, but might partly be responsible for observed deformation. The same applies to the shallow hydrothermal system in this area (Kanda et al., 2008). Anomalously low velocities, low resistivity and the presence of hot springs and a large amount of fracturing indicate the presence of geothermal systems in the uppermost $3 \mathrm{~km}$ below the caldera floor west of the central cones (e.g. Sudo and Kong, 2001; Asaue et al., 2006). Seismic anisotropy as well as observed deformation could be influenced by movement of fluid in those shallow parts of the volcanic system. In addition, we did not incorporate Kuju volcano, which is located northeast of Aso volcano. Studies have pointed out the presence of a possible magma body between the two volcanoes around station MKN (Sudo and Matsumoto, 1998) derived from $P$-velocity analyses and gravity data, as well as hydrothermal systems in the area around Kuju volcano in general (e.g. Kita et al., 2009). In addition, Kubotera and Mitsunami (1980) detected an earthquake swarm at the northern boundary of Aso caldera that appeared to be unrelated to volcanic activity at Aso volcano but which could be an indicator for fluids. The presence of hydrothermal fluids and/or magma unrelated to Aso volcano could account for stresses and create anisotropy.

- The simplicity of our models also only allows for one regional stress direction. However, differences in regional stress directions around the caldera do exist (Townend and Zoback, 2006): For our calculations we used an estimate of $S_{H, \max }=106^{\circ}$ from inside the caldera, which is similar to an estimate of $S_{H, \max }=112^{\circ}$ further northeast. Towards the southwest, faults and focal mechanisms indicate a maximum, horizontal compressive stress oriented $\mathrm{N} 75^{\circ} \mathrm{E}$. Allowing for this variation in the models could change the impact of 
stresses from local sources and change the fit to our observations.

- The source locations are only rough estimates based upon previous studies, with uncertainties on the order of kilometres. Tweaking source depths, location in the $\mathrm{x}-\mathrm{y}$-plane and volume might improve (or worsen) the fit considerably. Furthermore, the modelled strain is one to two magnitudes larger than the values calculated from GPS. In addition to varying the source parameters, detailed investigation of different sets of elastic parameters would be necessery to find models where both stress directions as well as strain values fit our observations from shear-wave splitting and GPS. Additionally, if for example viscoelastic processes occur, observed strain might be lower than the estimate calculated for an elastic halfspace. Unmodelled topography might have a further effect on reducing observed strain compared to the prediction modelled in Coulomb 3.1.

- The strain field is calculated from lowpass filtered data, so shallow short-term fluctuations due to small scale processes should at least partly be removed. On the other end of the spectrum, we did not account for the effects of possible slow slip on the subduction interface, that could slightly affect the region southeast of Aso volcano, or for co- and post-seismic displacements from larger earthquakes (Ozawa et al., 2001). Yet, no slow slip events or large earthquakes have been reported for the investigated area and time. Furthermore, we do not incorporate a possible contribution to strain from the mantle. It is possible that including those processes would results in a better fit of modelled stress and dilatation to the strain field.

- The fit calculation that determines the best fitting model assumes that 2D shortening axes align with maximum horizontal stress, which is only true if there is no intrinsic anisotropy (see section 2.2). Hence there is always an uncertainty about whether potential misfit of any given model is due to wrong modelling parameters or simply due to the presence of intrinsic anisotropy.

Furthermore, the fit of our models is dominated by the strain calculations. The main change in the deformation pattern in 2003 is represented in our models by a major change in stress pattern. Although we observe another period of variation in deformation at the end of 2004 coinciding with variations in shear-wave splitting parameters and $v_{P} / v_{S}$-ratio, we cannot easily infer a direct connection of these crustal elastic properties to the processes influencing the strain field. In fact, if inflation of the sill caused the change in fast direction in 2004, we would expect a similar change of $\phi$ in 2003. Seismic data for this time period are sparse, however, and we can neither confirm nor deny a direct relation between the observed seismic properties, strain and modelled stresses. 


\subsection{Gisborne}

\subsubsection{Influences on shear-wave splitting results}

Variations in shear-wave splitting parameters at this point do not show any systematic pattern that could be related to variations in stress. While fast directions at station MWZ, for example, are relatively stable, there are unexplained changes at station KNZ in 2009-2010 compared to 2002-2005. We could not find any parameter that could be related to the changes in $\phi$ (see figure 8.14). Furthermore, there is no obvious correlation of fast directions to the strike of active faults close to the stations. $\phi=5^{\circ} \pm 3^{\circ}$ at station PUZ for 2002-2005 is in agreement with a previous estimate of $\phi=6^{\circ}$ for events shallower than $100 \mathrm{~km}$, filtered with a long period filter $(0.1-1 \mathrm{~Hz}$, Audoine et al., 2004). The mean value of $\phi=-5^{\circ} \pm 5^{\circ}$ at station PUZ for 2009-2010 is not quite the same, but still very similar. In contrast, the results from a short period filter $(0.5-3 \mathrm{~Hz})$ in the study by Audoine et al. (2004) for the same depth range yield $\phi=33^{\circ}$, indicating a potential frequency dependence of splitting. A detailed analyses of frequency content of seismograms, different filters and a separation of splitting results from different subsets of earthquake could illuminate the cause for the discrepancy but is beyond the scope of this study. Furthermore, a change in shear-wave splitting fast direction of $31^{\circ} \pm 24^{\circ}$ (towards NNW and away from the trend observed by previous studies) from before to during slow slip is observed at station PUZ. However, we cannot detect the same change for any of the other analysed slow slip events. It remains unclear whether there is a relation or not. Again, further analyses of frequencies, depth distribution etc. could give insights into the variations.

\subsubsection{Deformation}

The GPS displacement vectors for the 2010 slow slip event agree well with other studies of slow slip events in this area (Wallace and Beavan, 2010) as well as with findings from other areas where slow slip has been observed such as Cascadia (e.g. Holtkamp and Brudzinski, 2010) or Japan (e.g. Ozawa et al., 2001): They point roughly towards the trench during the slow slip event, indicating movement of the upper plate towards the plate boundary, and away from it on the long term average. The same applies to the 2D strain field: While during times of strain accumulation the region is dominated by compressional areal dilatation due to the coupled nature of the plate interface for interseismic periods (e.g. Beavan and Haines, 2001; Beavan et al., 2008; Correa-Mora et al., 2009), the slow slip event itself provides release of accumulated strain (e.g. Schwartz and Rokosky, 2007) and leads to extensional dilatation. Accordingly, the shortening axes show a $90^{\circ}$ flip from trench normal preslow slip to trench parallel during the event. The post-slow slip strain field shows stronger heterogeneity compared to the field observed before the event (see figure 7.20); especially a northeast striking band of extension stands out. This period, however, covers a shorter time span, so it is possible that there is influence of the 

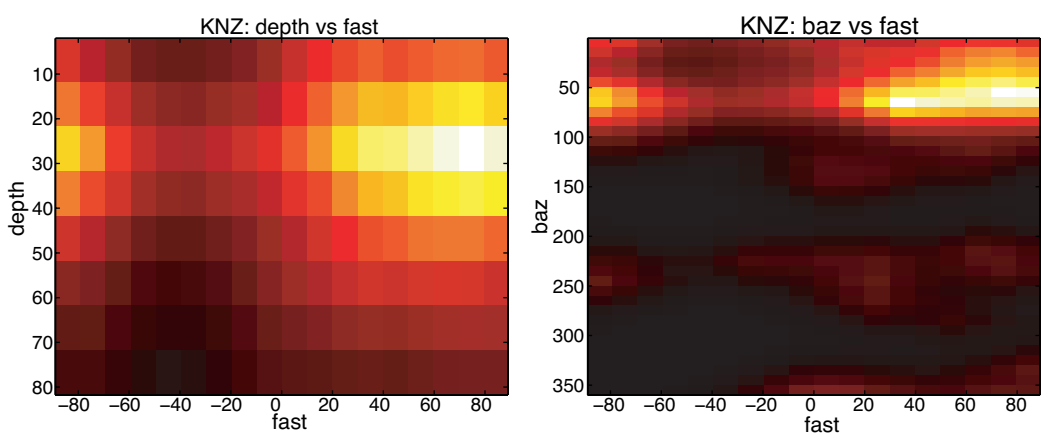

low point

density
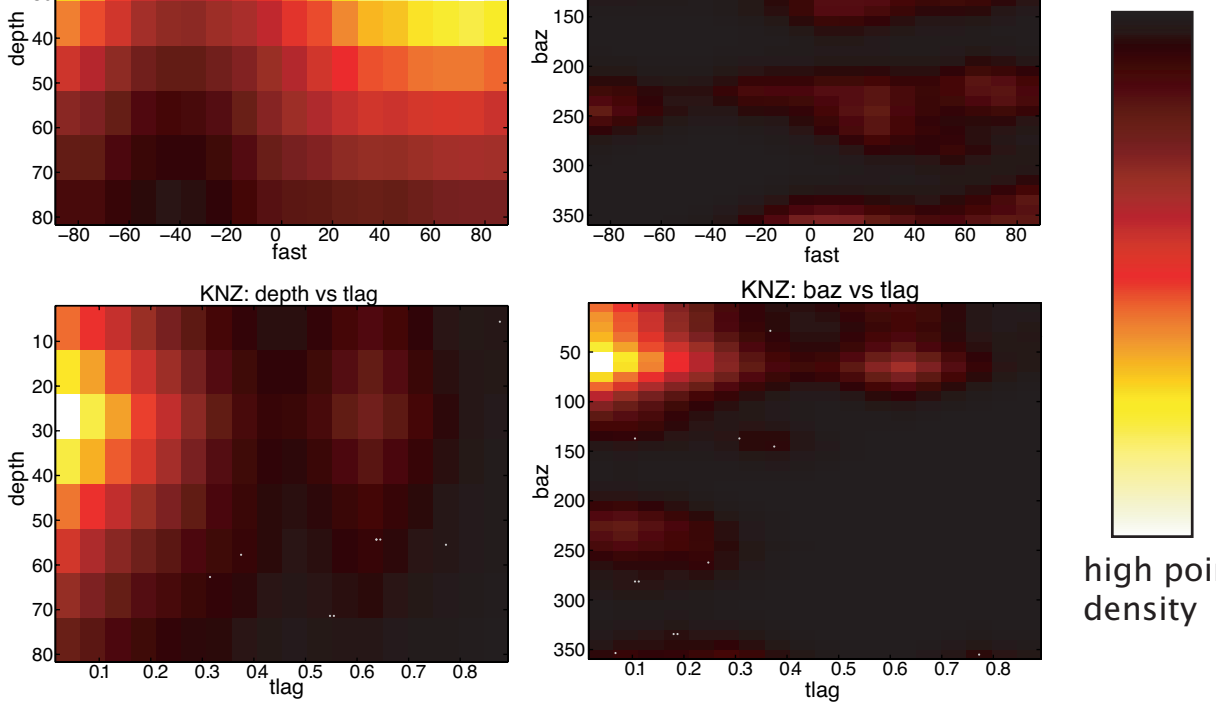

high point

density

(a) KNZ, 2009-2010
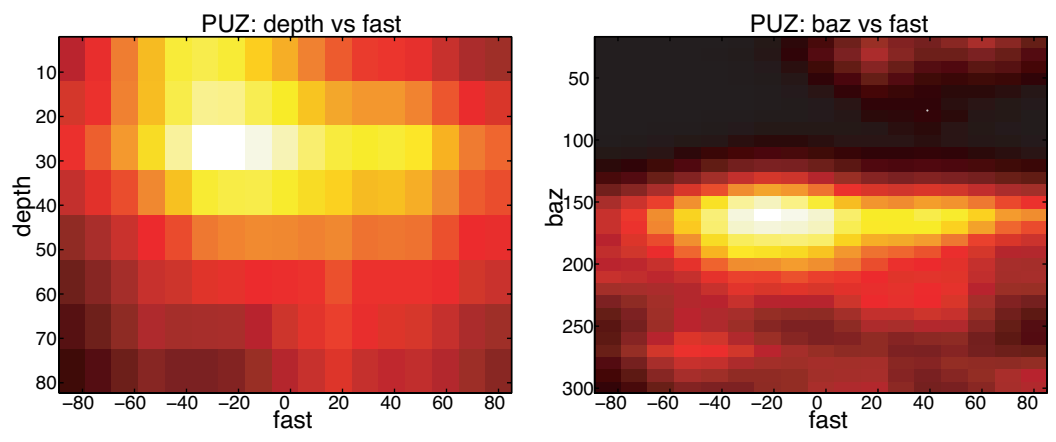

low point

density

PUZ: depth vs tlag

PUZ: baz vs tlag
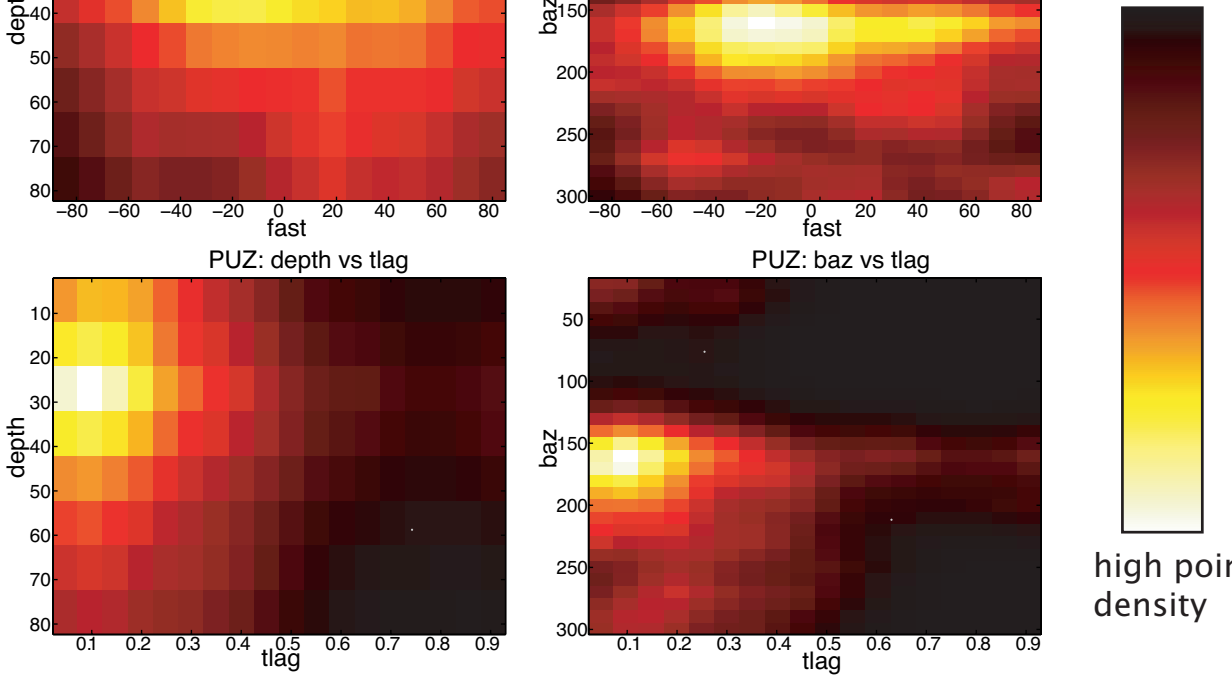

high point density

(b) PUZ, 2009-2010

Figure 8.14: Examples for dependencies of $\phi$ and $\delta t$ on earthquake parameters at two stations around Gisborne for 2009-2010. Colours indicate point densities. The white dots that show up in a few plots are artifacts from the density calculation. The upper row shows is for $\phi\left(\right.$ fast in $\left.{ }^{\circ}\right)$, the lower row for $\delta t$ (tlag, in seconds). The first column shows depth (in $\mathrm{km})$, and the second backazimuth $\left(b a z\right.$, in $\left.{ }^{\circ}\right)$. 
early onset of another, coming slow slip event. Alternatively, it could be that we see the fading of the slow slip event or that deformation could be due to more local processes on shallow faults etc. As the main scope of this study was the relation to shear-wave splitting, however, further discussion and interpretation is omitted here.

\subsubsection{Integrating GPS and shear-wave splitting}

Although we observe variations in shear-wave splitting, they are not strikingly correlated with the GPS time series. The fast directions agree with the strain shortening axes at some stations, but differ at others. As neither the stresses that cause strain nor structure seem to be related to the variations in shear-wave splitting, it remains unclear what controls those changes. It is possible that looking at different subsets of splitting measurements can illuminate the processes, as described in the discussion earlier and in the paragraph below. It should be noted, however, that the stations used here only cover the margins of the area investigated in terms of strain. Uncertainties close to the boundaries of the strain field as well as the fact that the actual slow slip event is most likely located in an offshore part of the subduction interface (Douglas et al., 2005; Beavan et al., 2008; Wallace and Beavan, 2010) make further research necessary before we can neglect contribution of slow slip processes to variations in shear-wave splitting in a reasonable way.

Focal depths In our analyses, we did not divide the data into different subsets for different depths due to time constraints. As described in section 4.3, however, we can expect to get very different contributions to splitting parameters from earthquakes from the sub-slab mantle, the slab itself, the mantle wedge and the overriding crust due to different stress regimes, mineral alignment etc. (McGinty et al., 2000; Greve et al., 2008). In addition, crustal depth across the Raukumara Peninsula varies significantly (Reyners et al., 1999), so for creating subsets one will also have to account for a backazimuthal variation in depth. An estimate for the approximate depth of the slow slip event on the subduction interface is $\sim 14 \mathrm{~km}$ (Douglas et al., 2005). If we look at a subset of earthquakes with ray paths going through the inferred region of slow slip, changes over time might be more clear. However, it is also possible that fast directions of the regional events used here mainly represent relatively shallow anisotropy (on the order of a few kilometres) and are hence only linked marginally to slow slip processes.

Strain field and fast directions The piercing points of the incoming earthquake rays at $5 \mathrm{~km}$ depth are calculated and the shear-wave splitting fast directions are projected onto those points (figure 8.15). Those directions are then used to calculate the fit to the grid of strain shortening axes. The fit is the cosine of the average residual angle. Interestingly, the splitting directions from 2009 and 2010 (see figure 7.18) during slow slip do not fit the relatively homogeneous strain fields (see figure $7.20)$ very well (0.521 and 0.488 , respectively). For the time post slow slip however, 
there is a surprisingly good fit of 0.814 . For this time the strain field shows high variations in both extensional vs. compressional dilatation and shortening axes over small distances (figure 7.20). We cannot exclude that the good fit is due to those high variations, i.e. a certain randomness in the strain directions that fits scatter and bimodal distributions of $\phi$. 


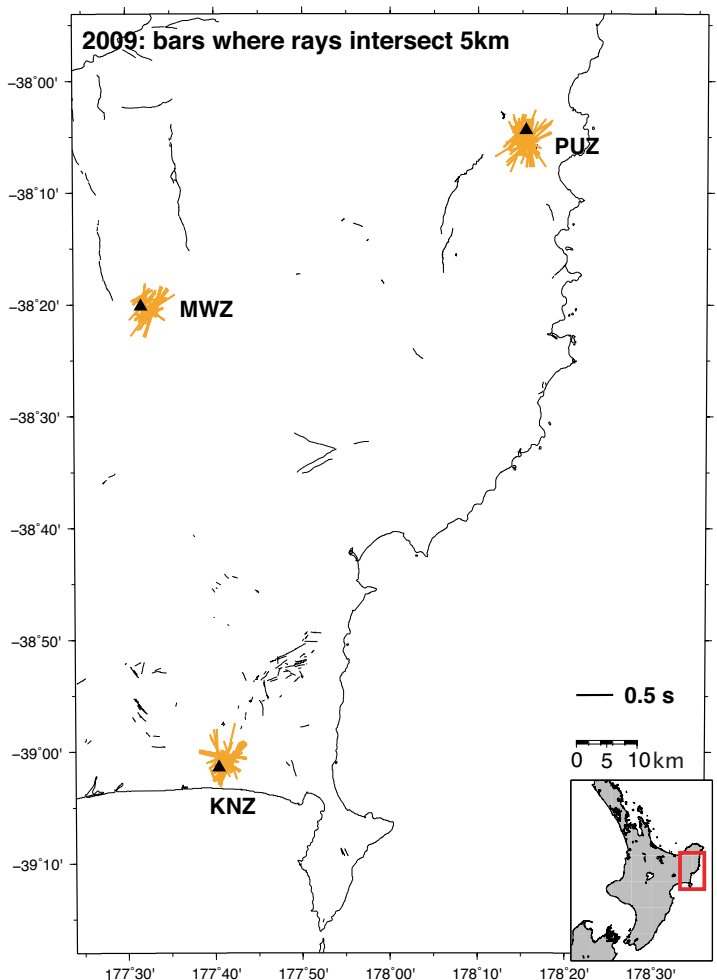

(a) 2009

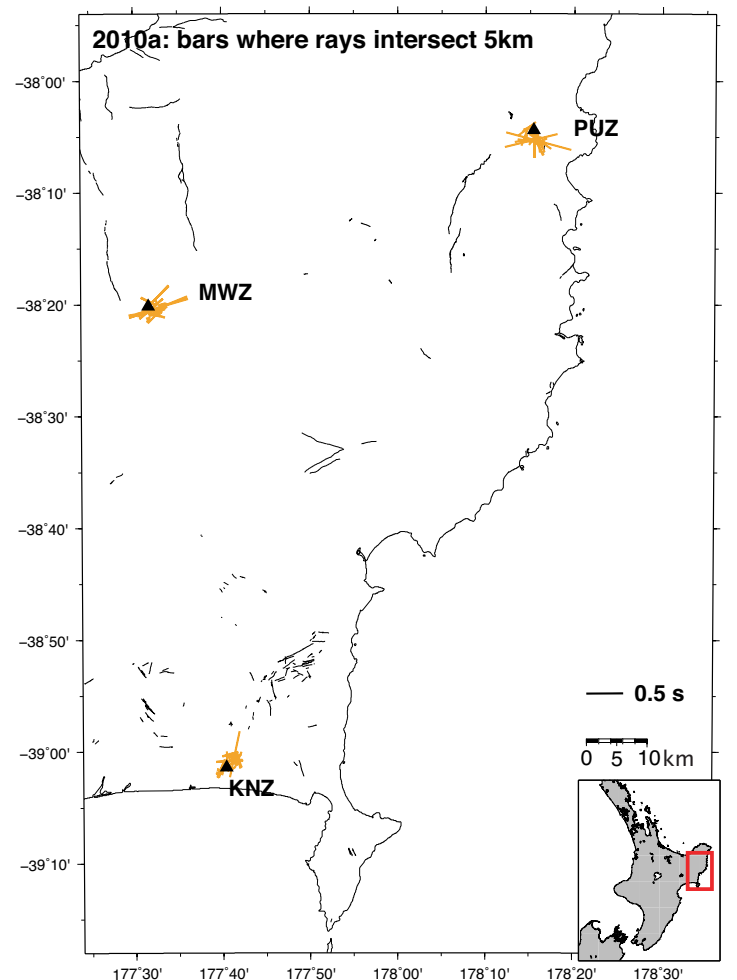

(b) 2010 during slow slip

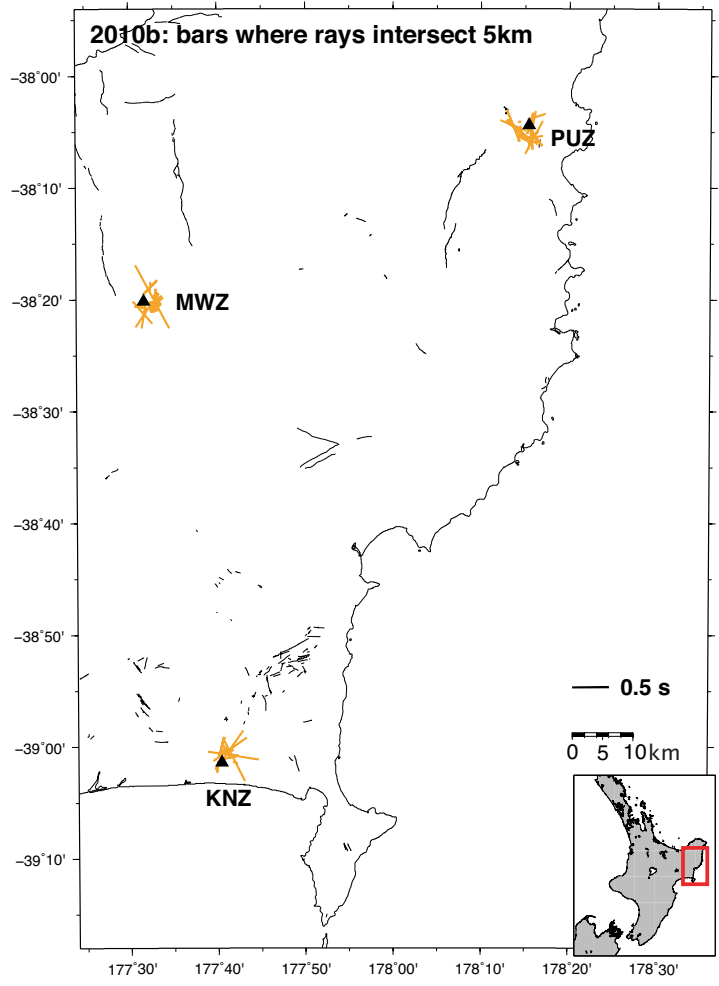

(c) 2010 after slow slip

Figure 8.15: Shear-wave splitting fast directions projected onto their intersections with an imaginary plane at $5 \mathrm{~km}$ depth, length scaled by delay time. 


\section{Conclusions}

\subsection{What did we learn from GPS and shear-wave splitting?}

The initial motivation behind this study, a straightforward correlation of time varying displacement/strain and shear-wave splitting parameters could not be found. However, we were able to show a general agreement of $\phi$ with maximum horizontal compressive strain directions, linked also to the regional maximum horizontal compressive stress directions. In addition, Aso and Taupo calderas seem to show an influence of structural anisotropy, where fast directions clearly line up with the strike of active faults instead of or in addition to $S_{H, \max }$.

In the very beginning of this thesis there were five questions asked which we tried to answer through our research. We go back and give an answer to each one of those questions individually.

\subsubsection{Do shear-wave splitting parameters change over time?}

In all three regions there are changes in shear-wave splitting parameters over time. While scatter in $\phi$ seems to be relatively high, the delay times in general appear a bit more stable. There are stations where variations in $\phi$ and $\delta t$ occur at the same time, while at others the two parameters show no apparent relation. This result is not unexpected: If changing stresses are responsible for variations in seismic anisotropy, it is easy to imagine a scenario where for example just the differential stress magnitude changes, and accordingly the delay time experiences variations, while the fast direction might be unaffected. Vice versa, if the differential stress directions but not the magnitude change in a material with a uniform distribution of randomly oriented cracks, a different subset of cracks will be open after the change, yielding a variation in fast direction but not in delay time. Without any other data, however, it is almost impossible to give constraints on the processes leading to the observed changes. In addition, we cannot exclude source and path effects as cause of apparent temporal changes around lake Taupo and the Raukumara peninsula. At Aso caldera, however, our analyses of earthquake clusters show that the observed changes are most likely real time variations.

\subsubsection{Do positioning data show temporal changes?}

The ground in all three regions is experiencing surface displacement to various extents and for different reasons. While the Raukumara peninsula as well as Taupo caldera show signs of dominantly regional deformation and act as relatively uniform blocks, Aso caldera shows a distinct contribution of a most likely volcanic source to the observed deformation field. Although GPS data in general have a scatter that should not be underestimated, the temporal variations in surface displacement 
are more pronounced and easier to interpret by themselves than the changes in shear-wave splitting parameters.

\subsubsection{Are these changes (if existent) correlated?}

None of the regions show a strong, direct correlation of $\phi$ or $\delta t$ and GPS time series or baselines in time. Yet, this result is arguable: At lake Taupo we did not find an immediately extractable, strong volcanic source of deformation and hence we do not have a solid base on which we could interpret variations in splitting parameters. Similarly, at Aso volcano there are gaps of several months in our splitting data, unfortunately right at the time when the strongest deformation is recorded. More work needs to be done on data from the Raukumara to separate potential different path effects on splitting results. Thus we can neither deny nor confirm a correlation with certainty.

\subsubsection{What can we infer about structure, local and regional stresses?}

The reason for the lack of correlation partly lies in the cause for the strain and at least a subset of the shear-wave splitting: local and regional stresses. Both strain and shear-wave splitting are influenced by the regional stress. Strain shortening axes as well as splitting fast directions line up with the regional maximum horizontal compressive stress over the entire networks, when they are averaged over relatively long time spans (on the order of months to years). The difference arises due to the local stresses: They will most likely be different at each seismometer in the network due to potential differences in underlying geology, the presence or absence of pore fluids etc. The splitting measurements indicate a relatively strong sensitivity to small scale processes confined to a small volume around the respective station. In contrast - even though we can look at individual time series - the GPS becomes really powerful as a network. Strain calculations allow advanced interpretations in terms of regional deformation. We suggest that, in order to be able to detect deformation due to the same local processes that cause variations in seismic anisotropy the GPS networks would need to be a lot denser. In addition, the local stresses causing variations in shear-wave splitting might not cause observable surface deformation. Furthermore, the importance of structural anisotropy is not to be underestimated. Similar to current work by Johnson et al. (2011, in prep.), we suggest that future studies incorporate the influence of structural anisotropy before looking at time variations in order to get correct averages over time.

To sum it all up, shear-wave splitting is sensitive to a combination of structure and local, intermediate and regional stresses and it is not easy to separate the different effects. GPS in the network configurations in this study is more suitable to interpret intermediate and regional scale stress variations. 


\subsubsection{Are there remarkable differences or similarities in the two data types for different volcanic and non-volcanic environments?}

Splitting as well as positioning data show potential time variations in all three regions, volcanic and non-volcanic, although only at Aso caldera have source and path effects been excluded. Delay times and fast directions at lake Taupo generally show variations with larger amplitudes than the other two areas. As mentioned before, a valid interpretation can only be made when effects from source and path on splitting measurements have been excluded. Thus we will not interpret the nature of the larger variations in splitting parameters for Taupo caldera. Another difference arises due to the sources of deformation: relatively more local deformation at Aso caldera as opposed to regionally widespread for Raukumara and a complicated, not easily separable combination of potentially local and regional deformation at lake Taupo. Furthermore, there is a difference in terms of additional constraints: Aso is an active volcano close to largely populated areas and hence a major point of research interest. A vast amount of studies with several different methods make interpretation relatively easy compared to the other two areas. Taupo has not shown any eruptions in recent history, and there are neither a large amount of studies nor any major "bursts" of activity that variations could be related to. For the Gisborne area there might be more information in the data, but due to time constraints we could not pursue this track any further. The Raukumara peninsula and the associated slow slip events are a relatively new field of research. Geoscientists are only starting to understand those events and there is a lot of potential for future studies continuing the work of this project.

\subsection{Insights into processes related to an eruption sequence at Aso volcano}

As explained above, due to the higher amount of other studies on Kyushu island, data from Aso volcano yielded the most interesting results of this study. We calculated shear-wave splitting parameters, $v_{P} / v_{s}$-ratios and the strain field over time and modelled stresses that could account for the observed time variations. We consider the following scenario as a possible explanation for the observations made at Aso volcano: The region around Aso caldera might have a high density of cracks with an aspect ratio on the order of 0.1 and overpressured gases and hydrothermal fluids take up pore space, which eventually leads to an extremely low average $v_{P} / v_{s}$-ratio of $1.65 \pm 0.09$, similar to findings from other areas and laboratory studies (e.g. Tatham, 1982; Lin and Shearer, 2009; Kato et al., 2010). A sill with remnant magma at $\sim 15 \mathrm{~km}$ depth northeast of the active central cones and a potential magma chamber to the southwest at $\sim 6 \mathrm{~km}$ depth that have been found by previous studies (e.g. Abe et al., 2010; Sudo and Kong, 2001) are cooling, compacting and releasing gases and/or fluids, causing deep low frequency earthquakes and a long-term subsi- 
dence observed at the surface. A sequence of ash eruptions and enhanced activity at Nakadake first crater has been observed and analysed by Aso Volcanological Laboratory (2007); Miyabuchi et al. (2008) and might have been triggered by a small intrusion of magma into the sill in 2003. The following deflation of the sill may have caused spatial and temporal fluctuations in gas release, explaining the different variations in shear-wave splitting parameters and $v_{P} / v_{s}$-ratios at various stations mainly in 2004 observed in this study. Our less well constrained strain field estimates from late 2004 and early 2005 point towards another, shorter period of inflation of the sill plus potential deflation of the magma chamber. The inflation could represent another small pulse of magma related to the eruption sequence following in 2005. In 2006-2007 there might be a period of inflation of the magma chamber. $v_{P} / v_{s}$-ratios remain more stable over this period than before, but show slight variations starting in 2007. This could indicate that magma from the sill is migrating into the magma chamber, increasing pressure and is potentially causing deformation over a relatively small area and for a short time. On a longer time scale fluids might redistribute over a wider region and affect the average $v_{P} / v_{s}$-ratios. Figure 9.1 illustrates the main principles of our interpretation. However, the extension signal in the strain field for the last period starting in 2006 is weaker than for 2003 and located more towards the west of the caldera and seismic data are getting more sparse, so we cannot exclude the possibility that the signal is unrelated to the volcanic processes in general or make any strong arguments for or against our intepretation for this period.

\subsection{Further work}

To close we want to give a few suggestions for further research. Some analyses concerning Aso volcano will be done as a continuation of this project in preparation for publication. Others are only directions for future work in the same regions or with the same method.

\subsubsection{Taupo caldera}

Extraction of a volcanic deformation signal As mentioned earlier the main limitation for Taupo caldera was the absence of a strong volcanic deformation signal. While we do not exclude the possibility of the existence of such a signal for the time period investigated here, detailed analyses of the GPS data has to be done in order to separate tectonic from volcanic and regional from local deformation in order to get better constraints on influences on shear-wave splitting, and to counterbalance the poor station coverage in terms of processes happening in the caldera.

Calculation of $v_{P} / v_{s}$-ratio If indeed a volcanic deformation signal is extracted, $v_{P} / v_{s}$-ratios might help to identify for example the presence of melt vs. water or 


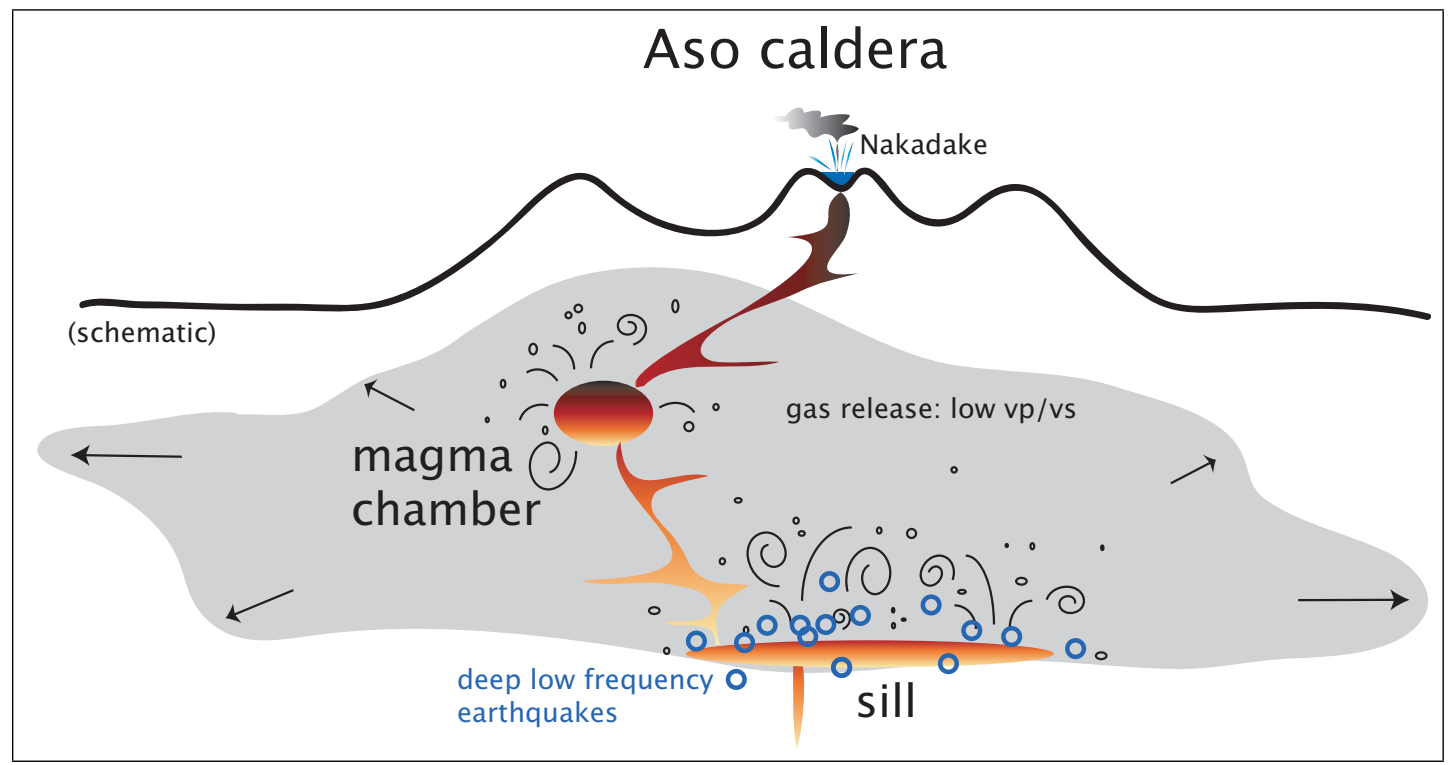

Figure 9.1: Schematic illustration of fluid circulation related to eruption sequence at Aso caldera. Small intrusion into sill triggered eruption sequence as well as observed surface inflation. Following deflation results in enhanced release of gases, and redistribution of fluids (also on longer timescales, grey area and black arrows) is responsible for fluctuations in $v_{P} / v_{S}$ and shear-wave splitting following the deformation signal. Generally, gas release from sill causing deep low frequency events (blue circles).

gases. In addition, calculating $v_{P} / v_{s}$ can give constraints on elastic moduli and hence improve potential stress modelling.

Extraction of similar events To exclude apparent temporal variations due to source or path effects further analysis is strongly advised. Earthquake clusters should be identified based upon source location and ideally similarity of waveforms, and analyses of temporal variations should then be restricted to the extracted subset of similar events.

\subsubsection{Aso volcano}

Strain rate A recent study by Hiramatsu et al. (2010, in prep.) has suggested that normalised delay times from shear-wave splitting show a proportionality to strain rate. Calculating variations in strain rates in time and space and comparing the results to changes in delay times in time and space (see section 9.3.4) could confirm this relation and contribute to the understanding of what processes cause seismic anisotropy.

Ray tracing and improved separation of splitting measurements Splitting measurements from this study have only been separated according to the hypocentral 
depth $^{43}$ of the respective earthquake. Ray tracing will help to identify the regions in the subsurface sampled by the events and facilitate a selection of events with ray paths travelling through regions of interest for publication of this work. This might even contribute to estimating regions with different amounts of anisotropy or fast directions.

\section{Using a different subset of splitting measurements to compare to stresses} For our modelling so far we used all splitting measurements to calculate the fit of the stress model. However, in fact we are concentrating on shallow processes, so the amount of splitting results used will be restricted for subsequent modelling. Initially, for example using only earthquakes with hypocentres at crustal levels could be an improvement. If the events can be divided into subsets according to their ray path, a more accurate picture of possible processes causing stress variations could be achieved.

Analyses of deep low frequency events The presence of deep low frequency events at the depth of the proposed deforming sill is an important point. Depending on abundance, timing, etc. of those events our interpretation stands or falls. We recently received hypocentre information for those events (Y. Abe, 2011, pers.comm.), which we plan to analyse.

\subsubsection{Raukumara peninsula}

The pilot character of this part of the project that we mentioned before should be stressed again here. We strongly suggest that shear-wave splitting and geodetic data from the Raukumara peninsula are revisited and examined in more detail. While it is likely that within the next few years more slow slip events and a better station coverage will yield more data to work with, even the data presented here potentially contains information that still has to be extracted. The following paragraphs as well as the next section summarise potential steps that should be undertaken ideally to understand the complex interactions in this area.

Ray tracing and improved separation of splitting measurements In this setting a good estimate of the subsurface regions sampled by the ray paths from the earthquakes which yield splitting measurements is particularly important. Different contributions to splitting could come from the crust of the Pacific plate and the subducting slab, the mantle below the slab, the mantle wedge above the slab and the crust of the Australian plate (see e.g. Greve et al., 2008). Separating those effects is crucial if time variations should be interpreted accurately and compared to time variations in GPS. Ray tracing could help to faciliate such a separation.

\footnotetext{
${ }^{43}$ Note that the earthquakes used here have been located by the Aso network. Locations of earthquakes outside of the network should ideally be left out due to the uncertainty associated with the location.
} 
Shear-wave splitting on more stations This study only used three seismometers from the National Network that had been installed since at least 2004. Since 2006, however, more stations were installed in regional networks across the peninsula. Data from those seismometers could help to identify regional vs. local time variations in $\phi$ or $\delta t$.

Baselines Even though baselines will not give significantly more information than the strain field, they have a stronger emphasis on time. Calculating sets of baselines on the Raukumara peninsula might enable the definition of shorter time periods for which strain could be compared to splitting and stresses could be modelled.

\subsubsection{Amendments to the method}

The following paragraphs summarise a few ideas for methods which could significantly improve the interpretation of shear-wave splitting in combination with GPS data in general and are partly already in progress.

Understanding the statistics of shear-wave splitting Shear-wave splitting data tend to show a large scatter in both $\phi$ and $\delta t$. Understanding the origin of this scatter will improve the distinction between real time variations, noise and processing artifacts. Research in this area is currently being undertaken in the Geophysics Department at Victoria University in collaboration with the Statistics Department (M. Savage, 2011, pers.comm.).

Delay time tomography Quantifying the spatial distribution of shear-wave splitting delay time magnitudes could significantly improve estimates of regions of high anisotropy vs. relatively isotropic areas and enable a better interpretation of temporal and spatial variations. Previous studies such as Pastori et al. (2009) and continuing work by J. Johnson (2010, pers.comm.) have already successfully used this technique and a general implementation into the automatic splitting method would improve the quality of future shear-wave splitting studies.

$v_{P} / v_{s}$ tomography Similarly a tomography of $v_{P} / v_{s}$-ratio has been done in various settings (e.g. Kurashimo and Hirata, 2004; Reyners et al., 2006; Chiarabba and Moretti, 2006). Identifying differences in $v_{P} / v_{s}$-ratio at depth together with anisotropy observations, earthquake hypocentres etc. could again improve the interpretation signficantly.

Advanced stress modelling Based upon initial fit calculations and estimation of periods with deflation vs. inflation, we will improve stress modelling by trying a broader range of source models in terms of source size, volume change, location and more. 
Last but not least, the pitfalls of Coulomb 3.1 for this particular purpose need to be overcome. Ideally, we would perform a joint inversion, but at least topography, viscoelastic processes or varying regional stress directions, for example should be included in the modelling to get a more complete picture. 


\section{A. Errors in strain fields}

The following figures show $2 \sigma$-errors (where $\sigma$ is the standard deviation) for each grid cell for the different time periods and configurations in the order Raukumara peninsula, Aso caldera and Taupo caldera. The errors are obtained from the eigenvalues of the covariance matrix due to the overdetermination of the inverse problem in SSPX as discussed in section 5.2.3 and do not take into account errors of the GPS time series. They represent errors of magnitude of areal dilatation and have no relation to the estimates of directions of principal strain.

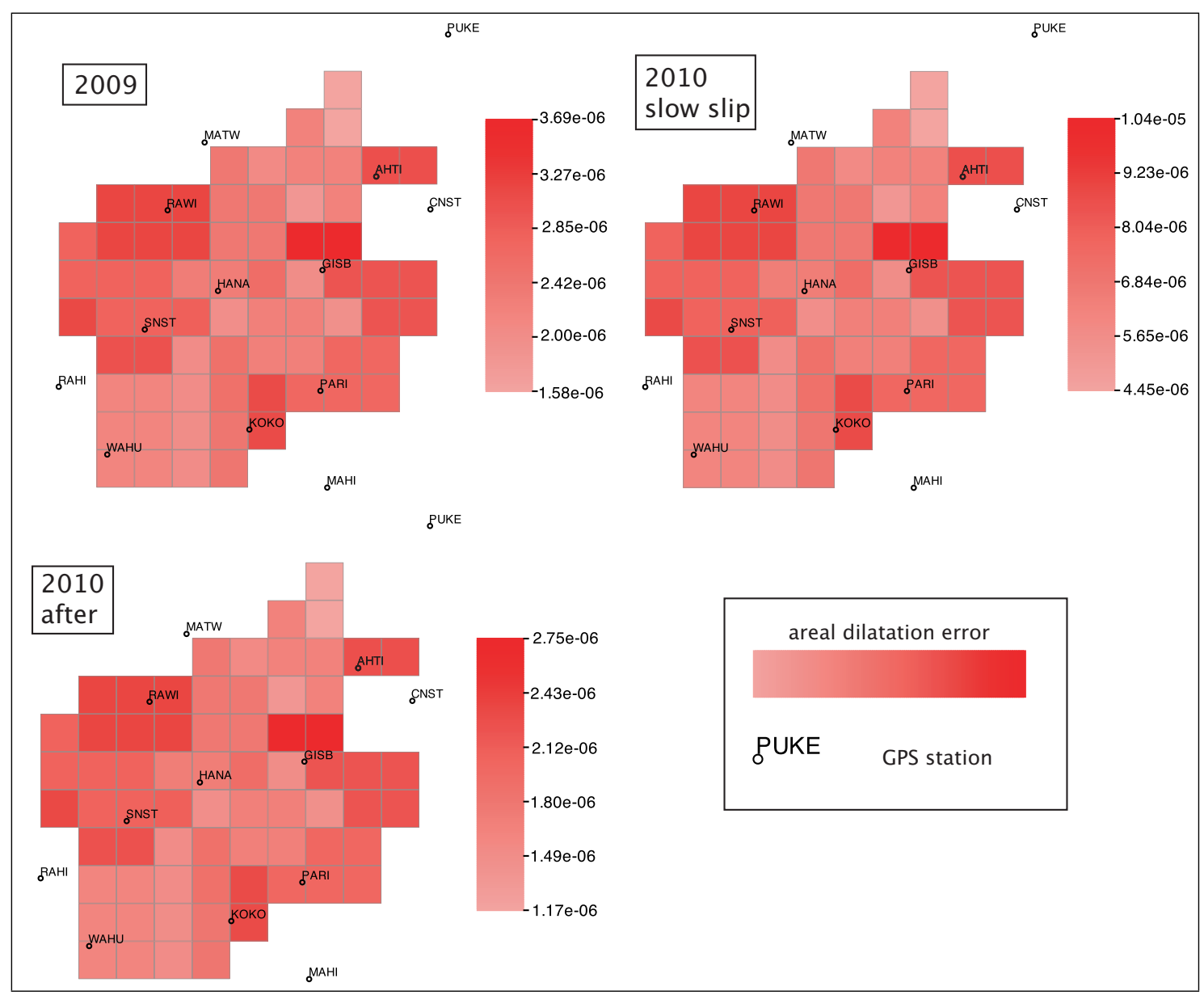

Figure A.2: Inversion errors from SSPX for Raukumara peninsula. Black dots with numbers are GPS stations, colours represent $2 \sigma$-errors of areal dilatation magnitude. Errors are on the order of $1-10 \%$ of dilatation magnitudes. 

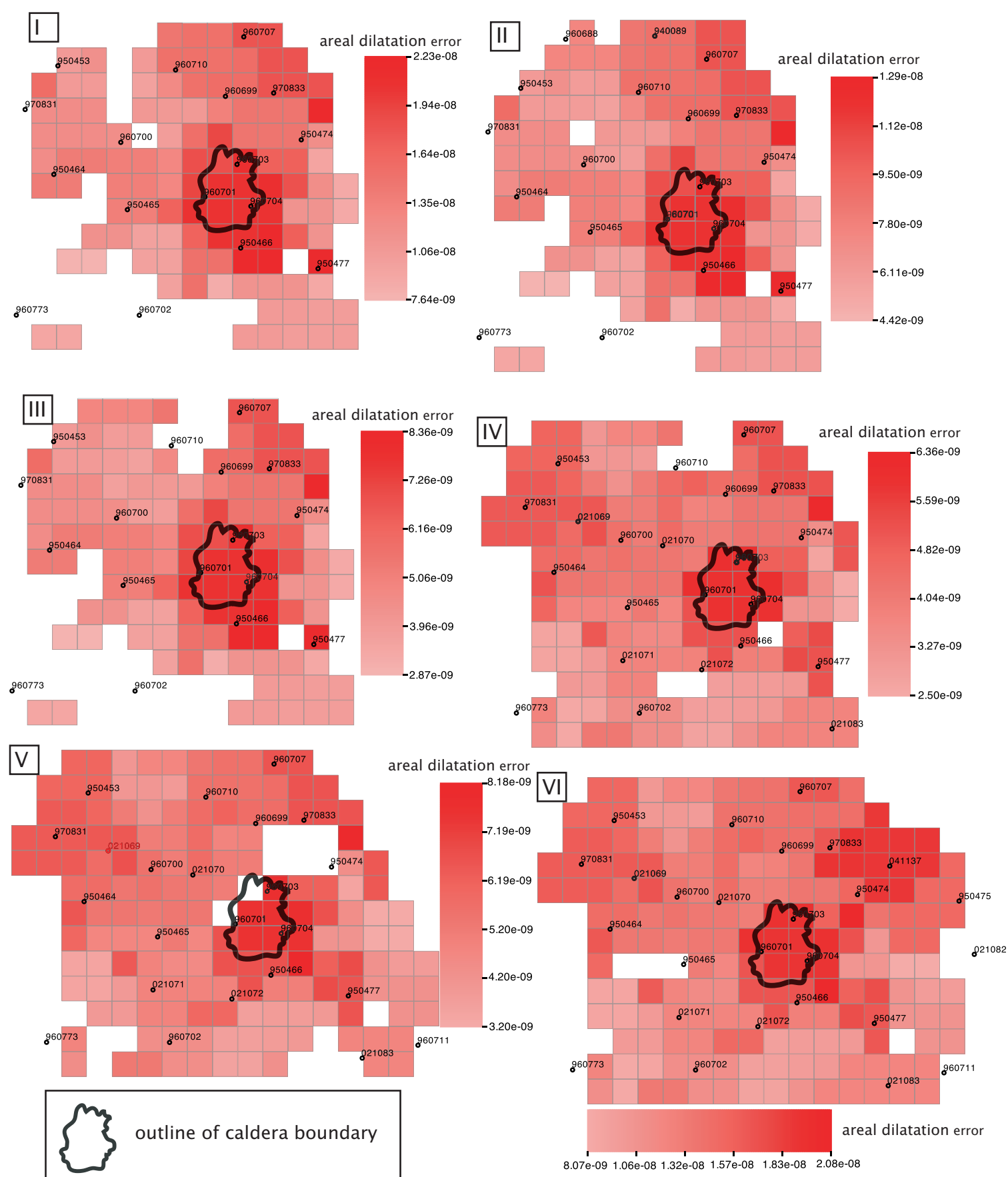

960711 GPS station

Figure A.3: Inversion errors from SSPX for Aso caldera. Black dots with numbers are GPS stations, colours represent $2 \sigma$-errors of areal dilatation magnitude. Errors are roughly $1-10 \%$ of the dilatation magnitude values. Caldera boundary outlined in black. 


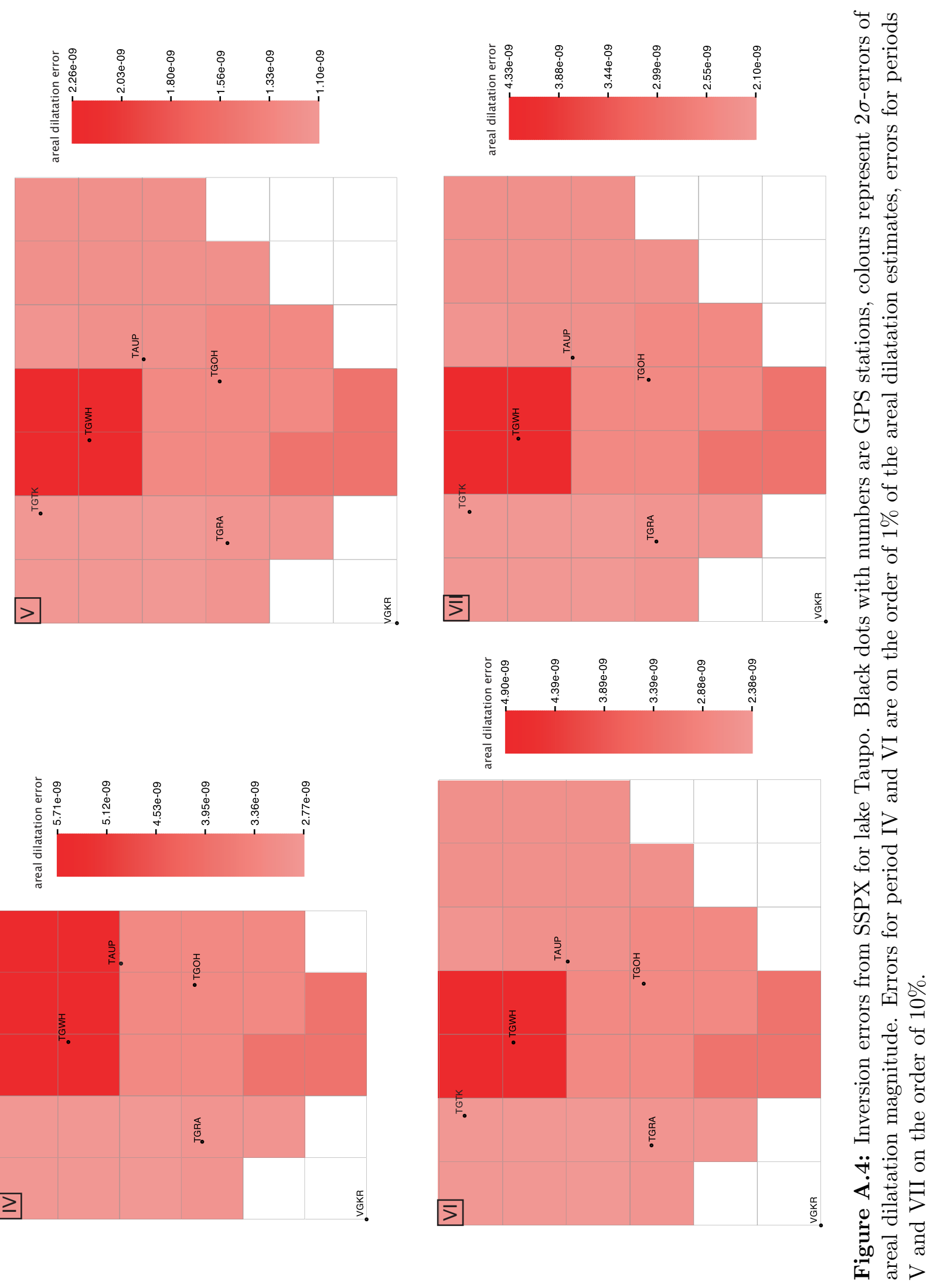




\section{B. Cross correlation of seismograms}

If an earthquake with a similar focal mechanism occurs at the same place over and over again, it can be used to exclude source effects as cause for appararent temporal changes in seismic anisotropy. To find repeating earthquakes the Bispectrum Crosscorrelation package for SEISmic events (BCSEIS) by Du et al. (2004) was used for earthquakes at lake Taupo.

The software uses seismograms in SAC-format (Goldstein and Snoke, 2005) as input. The data are cut and resampled and must contain event- and pick-information in the header. BCSEIS then filters all data with a user-defined filter and, after extracting station- and event-information, performs a cross-correlation of waveforms. In this step each earthquake pair undergoes all in all four cross-correlations, in the time and the frequency domain for both the unfiltered and the filtered data. Table B.1 summarises the input parameters we used here.

\begin{tabular}{|l||c|c|}
\hline parameter & most strict value & least strict value \\
\hline \hline cutoff distance between waveforms & $5 \mathrm{~km}$ & $30 \mathrm{~km}$ \\
\hline $\begin{array}{l}\text { number of sample points before } P \text { - } \\
\text { arrival }\end{array}$ & 30 & 30 \\
\hline number of sample points after $P$-arrival & 224 & 224 \\
\hline $\begin{array}{l}\text { number of sample points before } S \text { - } \\
\text { arrival }\end{array}$ & 50 & 50 \\
\hline number of sample points after $S$-arrival & 332 & 332 \\
\hline $\begin{array}{l}\text { number of sample points in each seg- } \\
\text { ment when dividing } P \text {-window }\end{array}$ & 64 & 128 \\
\hline $\begin{array}{l}\text { number of sample points in each seg- } \\
\text { ment when dividing } S \text {-window }\end{array}$ & 128 & 0.1 \\
\hline $\begin{array}{l}\text { lowest frequency to perform phase } \\
\text { spectrum fitting }\end{array}$ & 0.1 & 10 \\
\hline $\begin{array}{l}\text { highest frequency to perform phase } \\
\text { spectrum fitting }\end{array}$ & 10 & none \\
\hline $\begin{array}{l}\text { local velocity model for theoretical } S \text { - } \\
\text { arrival calculation }\end{array}$ & none & 0.95 \\
\hline lower cross-correlation coefficient limit & 0.7 & 0.98 \\
\hline centre cross-correlation coefficient limit & 0.8 & 0.99 \\
\hline upper cross-correlation coefficient limit & 0.9 & \\
\hline
\end{tabular}

Table B.1: Input parameters for BCSEIS, for the most and least strict runs.

While the values that were kept constant (see table B.1) had been successfully used to find repeating earthquakes at Okmok volcano (J. Johnson, 2010, pers.comm.), the other parameters were adjusted for lake Taupo: The initially used cutoff distance of $30 \mathrm{~km}$ gave earthquakes with high cross-correlation coefficients on mainly two stations with hypocentres which were tens of kilometres apart from each 
other and hence not suitable to find repeating earthquakes for the elimination of path effects. The similarity in waveforms for these events might be an effect of scattering structures or a noise source in the immediate vicinity of the station. Reducing the cutoff distance to a minimum of $5 \mathrm{~km}$ based on the approximate uncertainty of catalogue locations decreased the spread of events, but instead yielded mainshockaftershocks sequences and earthquake swarms with durations of only a few days, which are not suitable to detect time variations in seismic anisotropy. The different limits for cross-correlation coefficients were tested without success to improve the amount of correlated earthquakes.

We conclude that the method BCSEIS is not practical to find earthquake clusters for our purposes in the area around Taupo caldera. The four-fold correlation seems to be too "strict" for our target earthquakes or to be influenced too much by nearreceiver effects. It is possible that a different set of parameters to perform the cross-correlation would improve the results, but due to time constraints we did not pursue this path.

\section{Correlation of splitting parameters}

20 parameters related to the splitting measurements for all stations at lake Taupo were tested for correlation with each other. The following figures show examples of correlation and p-value matrices from MatLab for stations ARAZ and HATZ (for discussion of results see section 8.1.1). Parameters are abbreviated as listed in table C.2. Orientation parameters have been converted into the complex quantities to account for the fact that for example values of $1^{\circ}$ and $179^{\circ}$ are in fact almost the same orientation (E. Smith, 2010, pers.comm.). 


\begin{tabular}{|l|l|l|l|}
\hline evla: & event latitude & tlag: & delay time \\
\hline evlo: & event longitude & Dtlag: & error in delay time \\
\hline dist: & event-station distance & varfast: & complex fast direction \\
\hline depth: & event depth & fast: & fast direction \\
\hline mag: & event magnitude & Dfast: & error in fast direction \\
\hline baz: & $\begin{array}{l}\text { backazimuth at sta- } \\
\text { tion }\end{array}$ & anginc: & angle of incidence \\
\hline varspol: & $\begin{array}{l}\text { complex initial } S- \\
\text { polarisation direction }\end{array}$ & filthi: & $\begin{array}{l}\text { high corner frequency } \\
\text { of bandpass filter }\end{array}$ \\
\hline spol: & $\begin{array}{l}\text { initial S-polarisation } \\
\text { direction }\end{array}$ & spol-fast: & $\begin{array}{l}\text { initial polarisation di- } \\
\text { rection - fast direction }\end{array}$ \\
\hline Dspol: & $\begin{array}{l}\text { error in initial } S- \\
\text { polarisation direction }\end{array}$ & lambda: & $\begin{array}{l}\text { highest eigenvalue of } \\
\text { covariance matrix }\end{array}$ \\
\hline SNR: & $\begin{array}{l}\text { signal to noise ratio } \\
\text { ndf: }\end{array}$ & $\begin{array}{l}\text { number of degrees of } \\
\text { freedom for error es- } \\
\text { timation of splitting } \\
\text { measurements }\end{array}$ \\
\hline
\end{tabular}

Table C.2: Abbreviations for parameters from splitting measurements used for correlation 


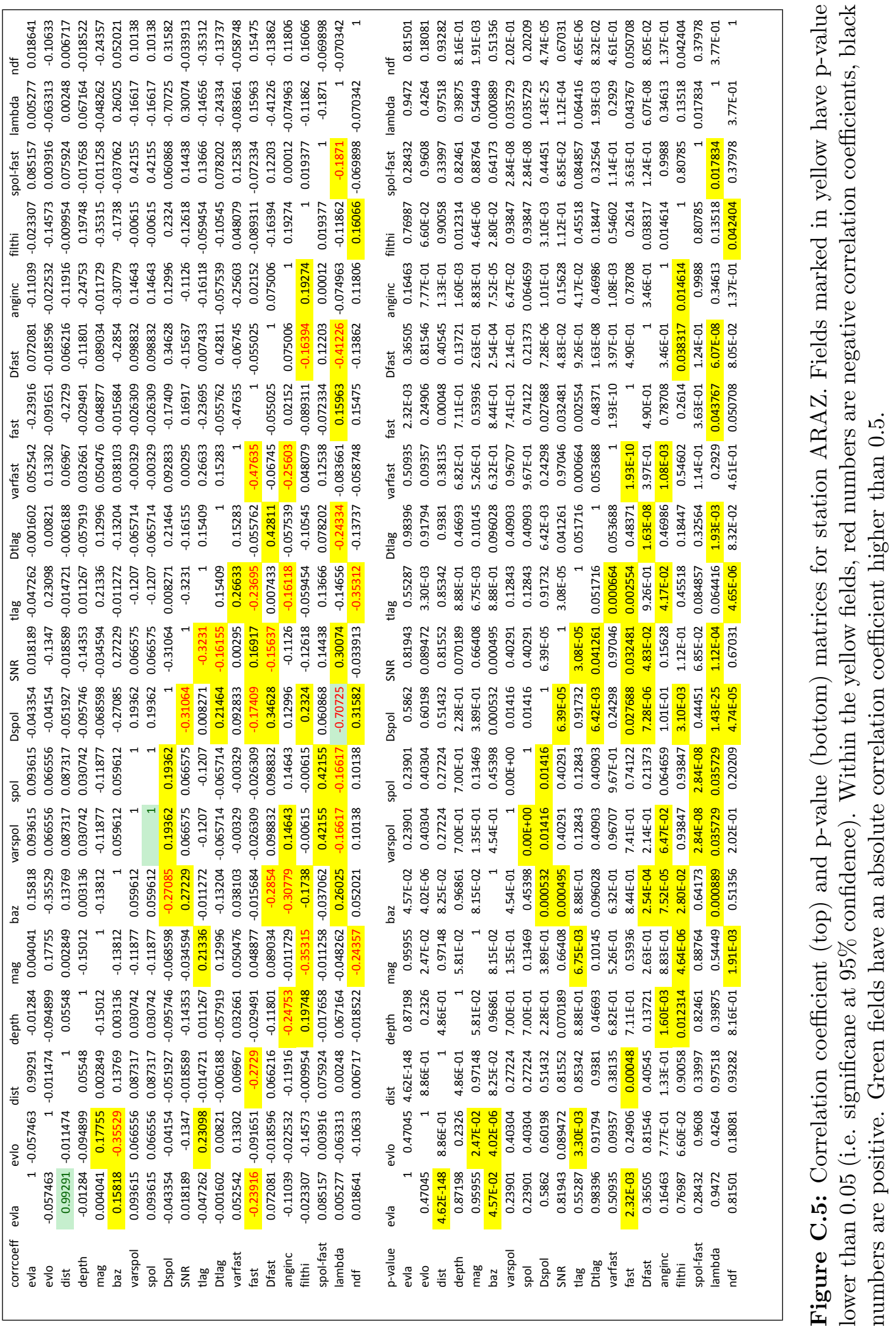




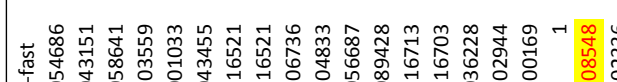

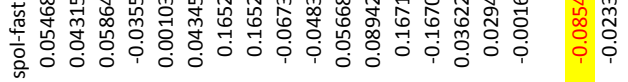

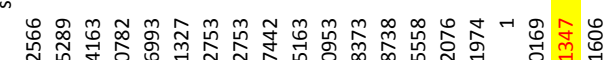

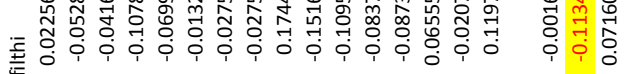

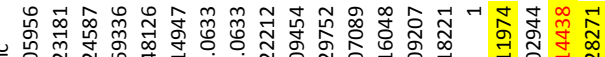

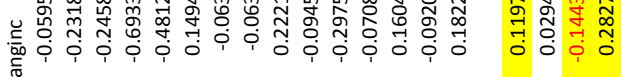

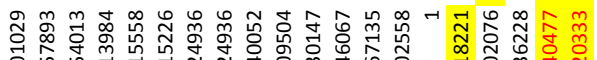

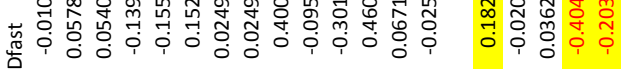

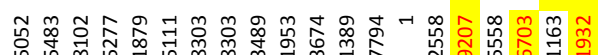

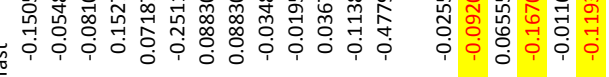

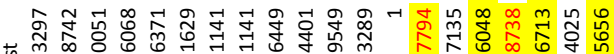

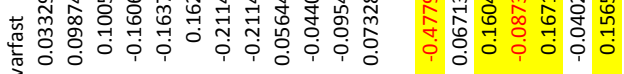

ర్ర

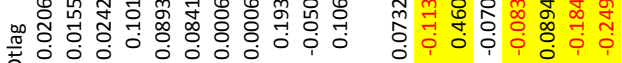

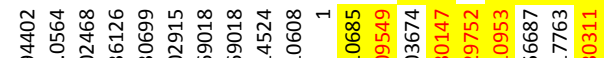

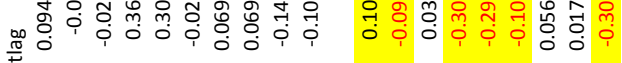

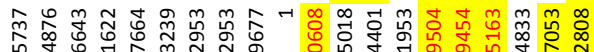

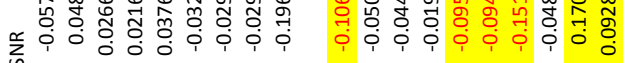

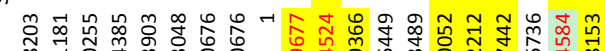

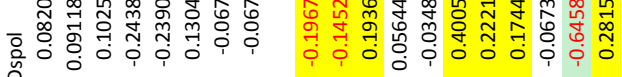

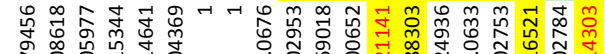

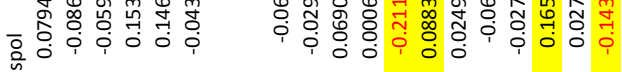

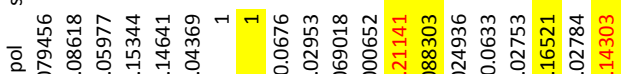

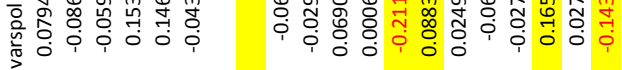

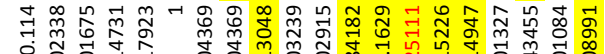

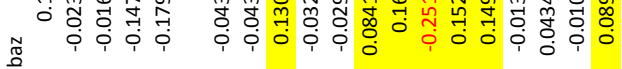

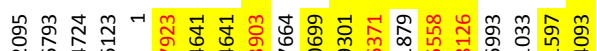

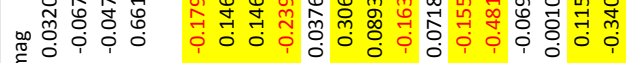

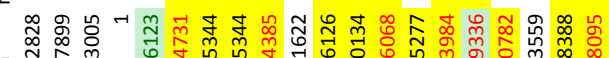

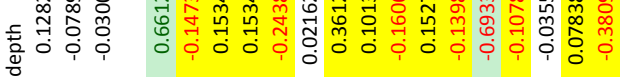

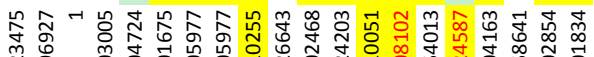

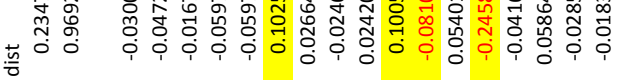

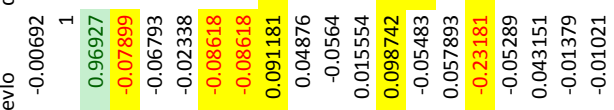

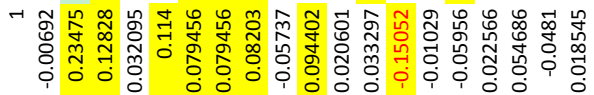

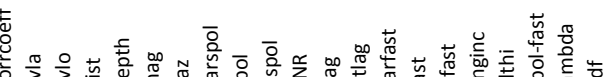

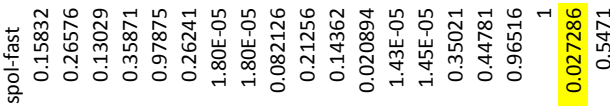

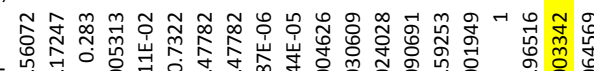

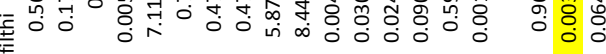

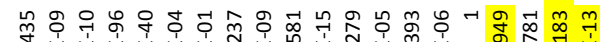

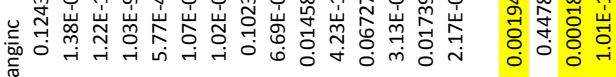
œ

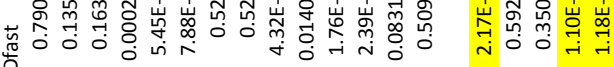

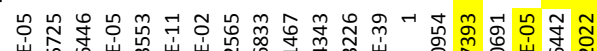

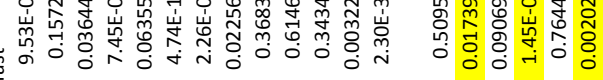

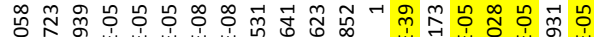

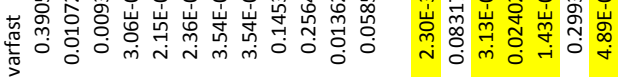

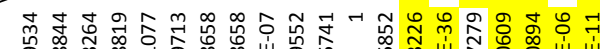

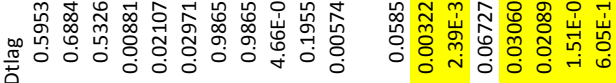

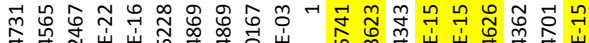

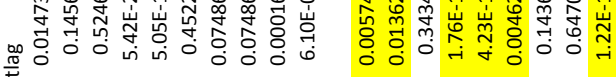
点

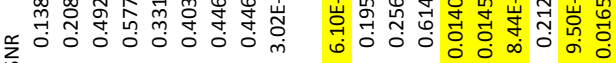

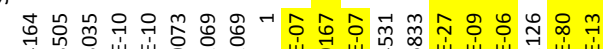

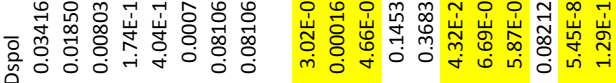

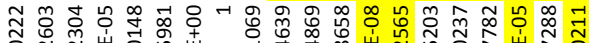
र $\begin{aligned} & 0 \\ & 0\end{aligned}$

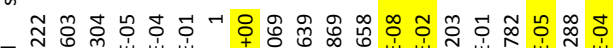

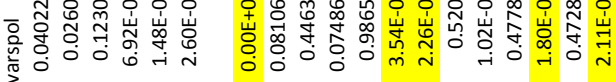

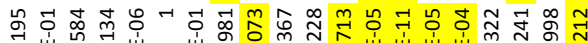

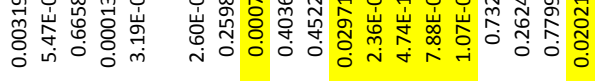

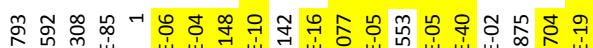

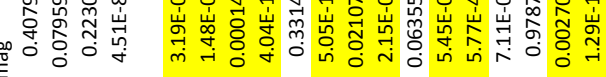
ஓ ₹

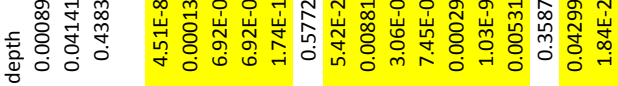

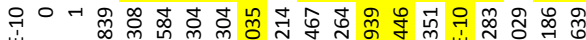

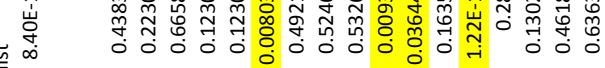

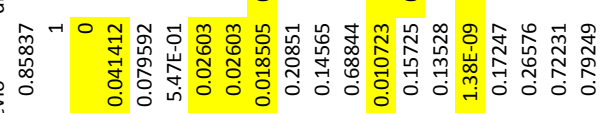

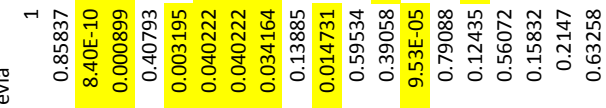

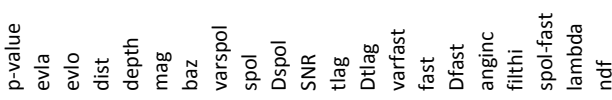

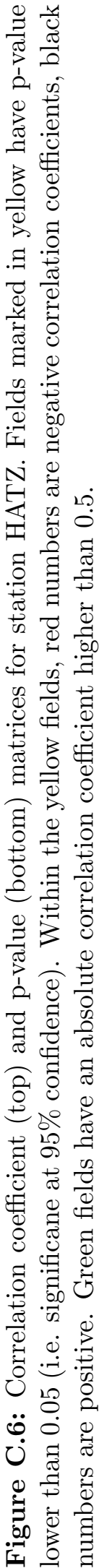




\section{Moving means at Aso caldera}

The following figures show 30 point moving means over $v_{P} / v_{S}$-ratio and $\delta t$ and $\phi$ from shear-wave splitting at Aso caldera for the six stations that have not been shown in section 7.2 (see figure 7.11 for station TAT). Grey dots are individual $\phi$ and $\delta t$, orange dots and blue dots are 30 point moving mean over $\phi$ and $\delta t$, respectively (all for events shallower than $35 \mathrm{~km}$ ). Red dots are individual $v_{P} / v_{S}$-ratio values and black dots are 30 point moving mean over $v_{P} / v_{S}$-ratio. Error bars are $95 \%$ confidence intervals. Note that the error bars on the moving means are strongly correlated, since only every 30 th point is independent. Also note the different scale for $\phi$ at station NBR. For station locations refer to figure 6.5. 


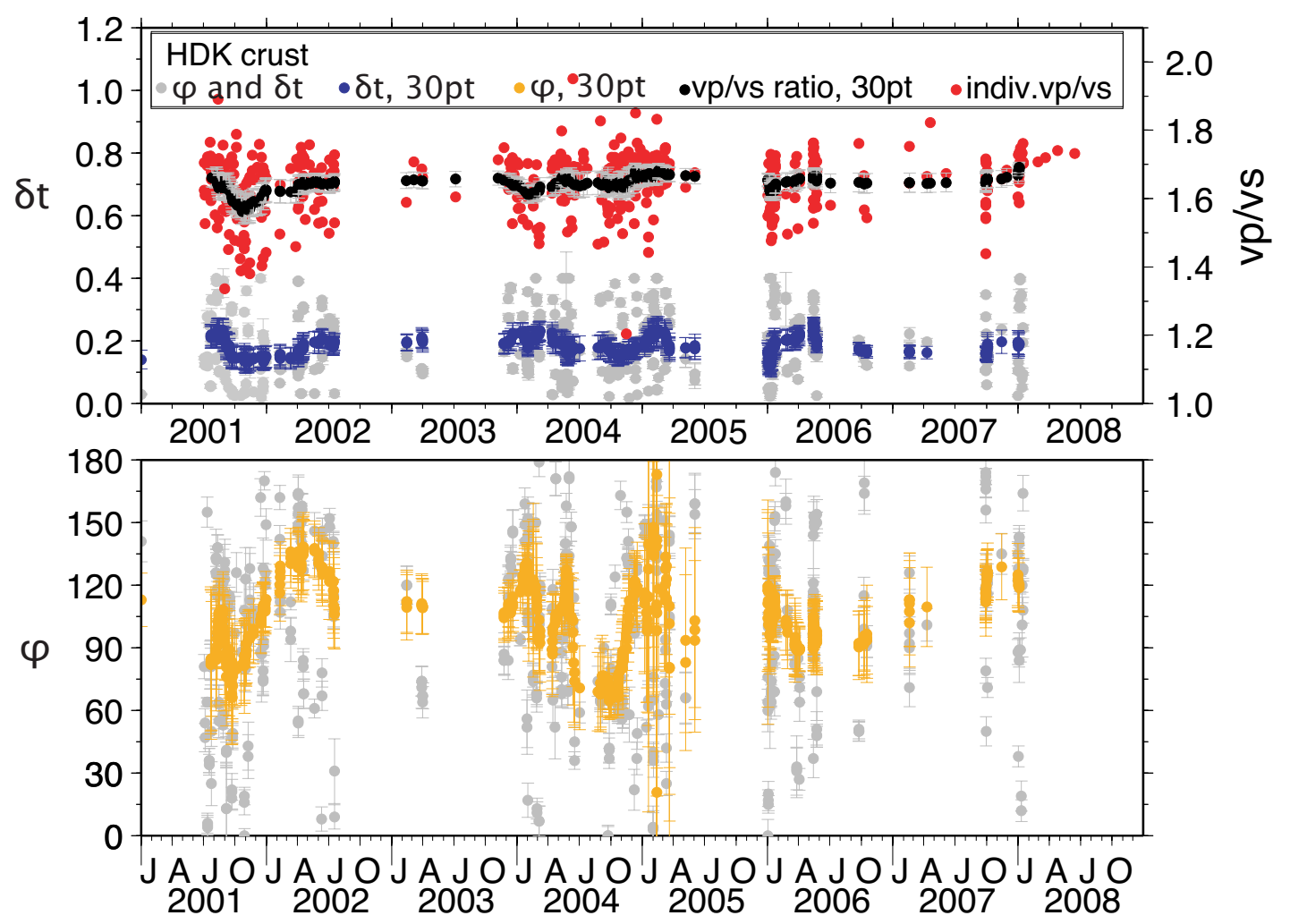

(a) HDK

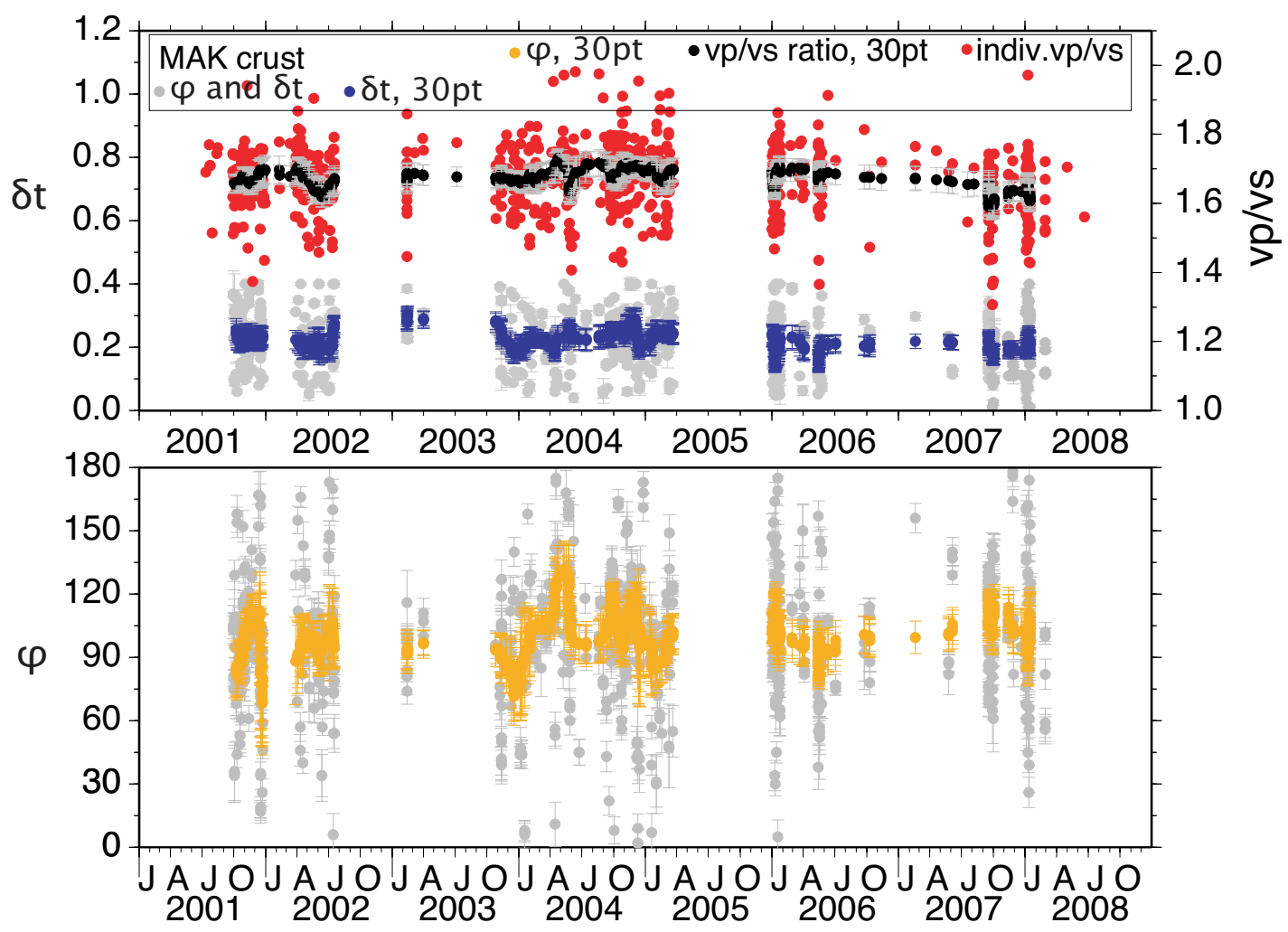

(b) MAK

Figure D.7: 30 point moving means at Aso caldera: HDK and MAK. 


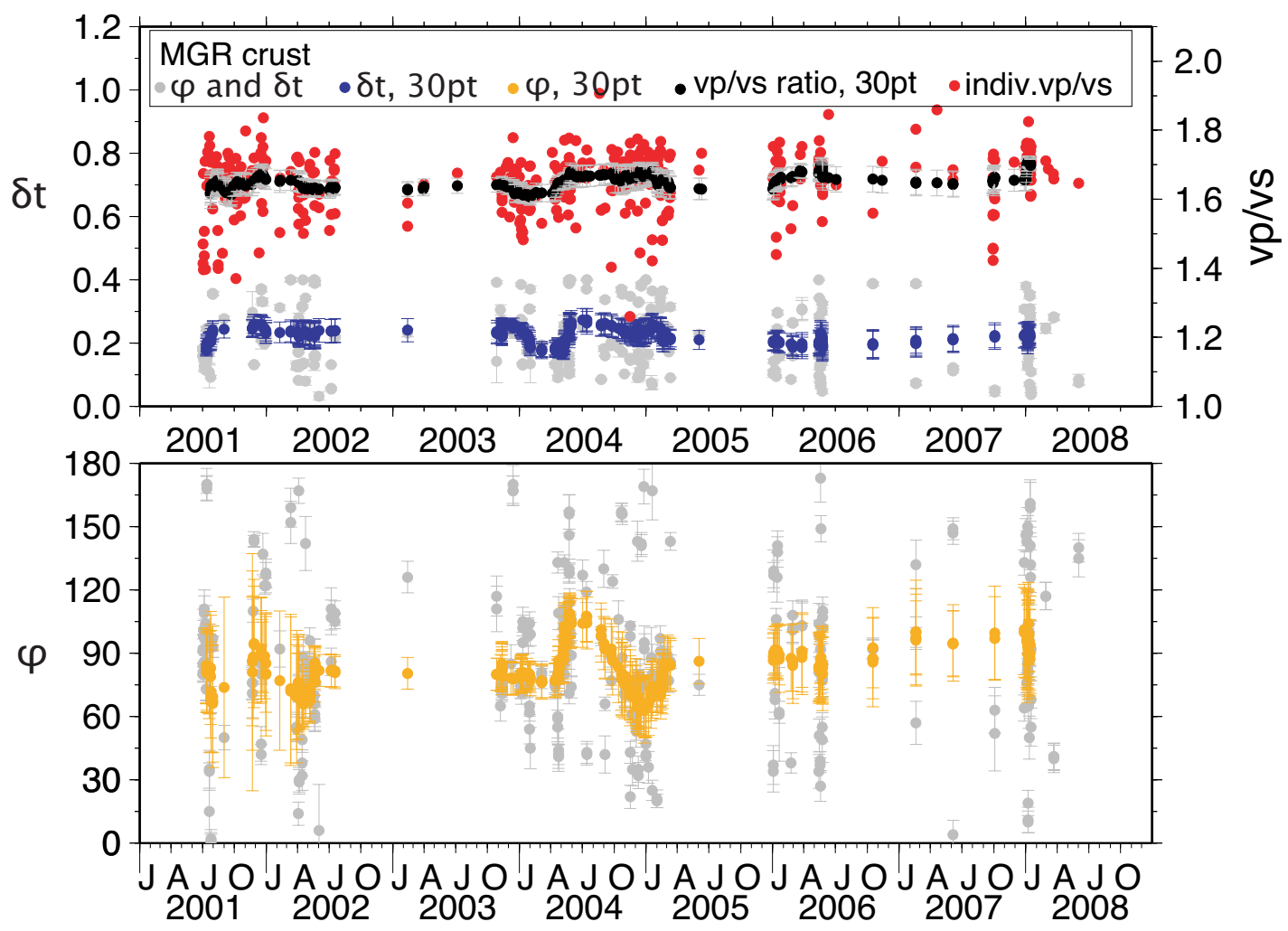

(a) MGR

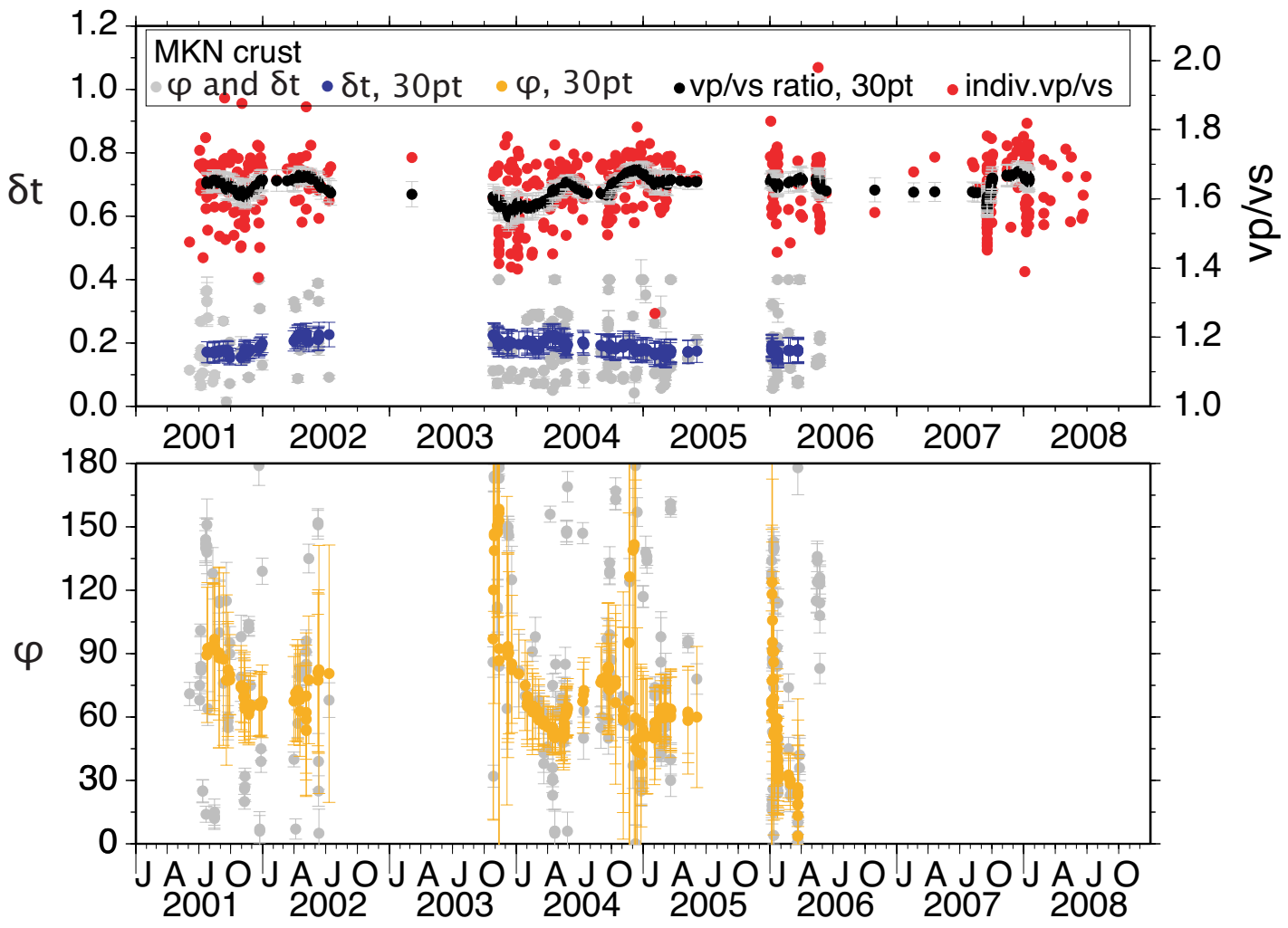

(b) MKN

Figure D.8: 30 point moving means at Aso caldera: MGR and MKN. 


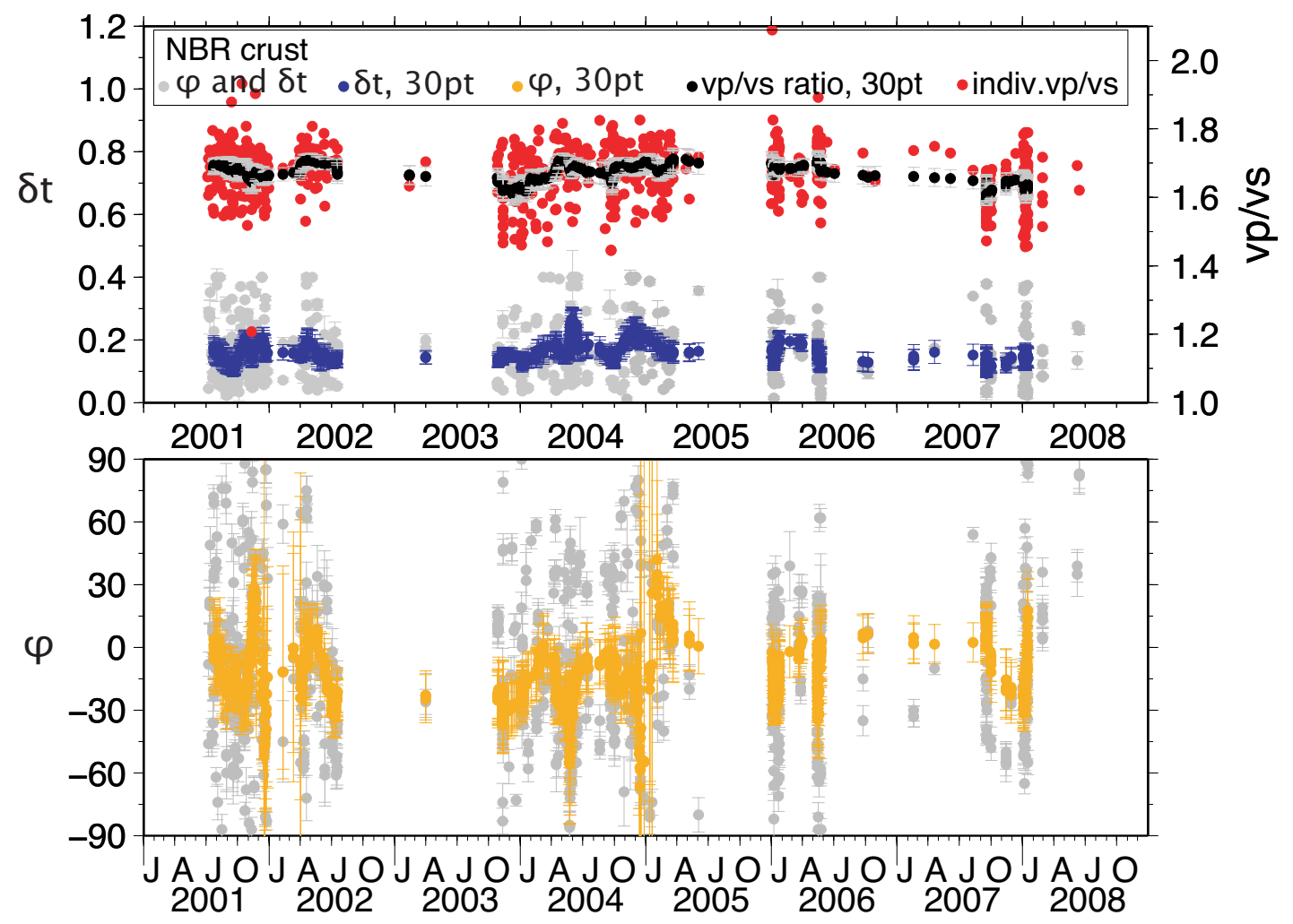

(a) NBR

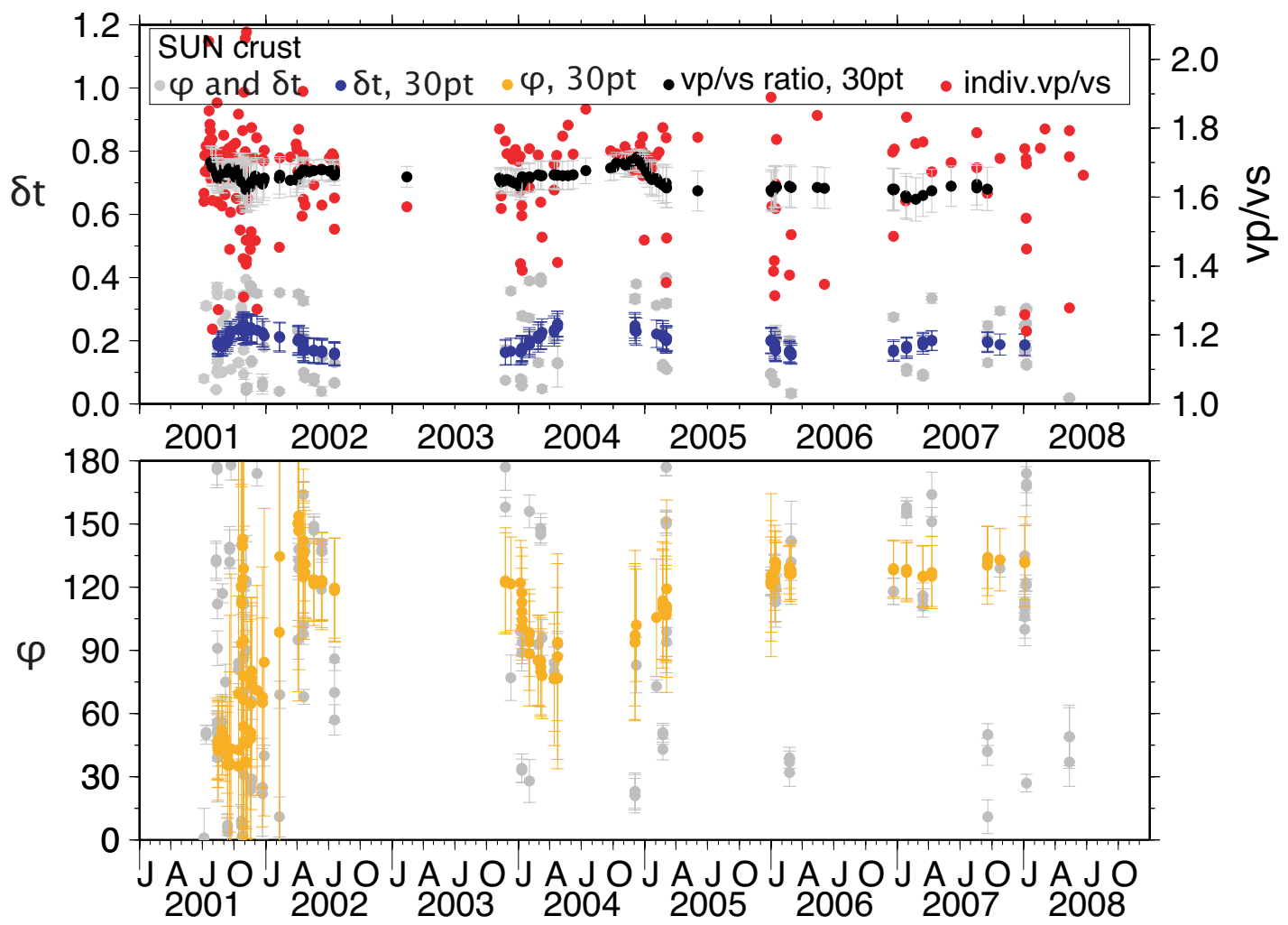

(b) SUN

Figure D.9: 30 point moving means at Aso caldera: NBR and SUN. 


\section{E. Calculations for stress gradient}

To validly model potential stress changes due to a volcanic (or any) source an estimate of the regional stress field is necessary. Particularly the differential stress between the minimum and maximum principal stresses influences whether a stress source will cause a change or not. Therefore, for stress modelling at Aso caldera we apply the calculations from Zoback and Townend (2001) for critically stressed faults in normal faulting regimes and parameter estimates from Brace and Kohlstedt (1980) as well as stress directions from Townend and Zoback (2006) as follows:

$$
\Delta S=S_{1}-S_{3}=\rho g z \frac{(\lambda-1)(1-F)}{F}
$$

where

$S_{1}$ : magnitude of maximum principal stress

$S_{3}$ : magnitude of minimum principal stress

$\rho$ : mean rock density

$g$ : gravitational acceleration

$z: \quad$ depth

$\lambda: \quad$ ratio of pore pressure over total vertical stress

$F: \quad$ ratio of the differences between $S_{1}$ and pore pressure, and $S_{2}$ and pore pressure.

With an estimate for $\rho=2600 \frac{\mathrm{kg}}{\mathrm{m}^{3}}$ derived as an average of densities for rocks at Aso caldera after Okubo and Shibuya (1993), $g=9.81 \frac{\mathrm{N}}{\mathrm{kg}}, z=1 \mathrm{~km}, \lambda=0.4$ (for near hydrostatic conditions, after Brace and Kohlstedt, 1980) and with coefficient of friction $\mu=0.8$ (after Zoback and Townend, 2001, and references therein) we obtain $\mathrm{F}$ from

$$
F=\left(\sqrt{\mu^{2}+1}+\mu\right)^{2}
$$

and hence estimate $\Delta S \sim 118$ bar $(11.8 \mathrm{MPa})$. With $S_{1}=\rho g z$ and the same values as mentioned above we estimate $S_{1}=255$ bar $(25.5 \mathrm{MPa})$, accordingly $S_{3}=137$ bar $(13.7 \mathrm{MPa})$ and $S_{2}$ in the middle at $196 \mathrm{bar}(19.6 \mathrm{MPa})$ at $1 \mathrm{~km}$ depth. Assuming all stresses equal to 0 at the surface, this leaves us with gradients for $S_{1}, S_{2}$ and $S_{3}$ of $255 \frac{\mathrm{bar}}{\mathrm{km}}\left(25.5 \frac{\mathrm{MPa}}{\mathrm{km}}\right), 196 \frac{\mathrm{bar}}{\mathrm{km}}\left(19.6 \frac{\mathrm{MPa}}{\mathrm{km}}\right)$ and $137 \frac{\mathrm{bar}}{\mathrm{km}}\left(13.7 \frac{\mathrm{MPa}}{\mathrm{km}}\right)$, respectively.

For near lithostatic conditions $\lambda=0.9$, from which we can obtain our low differential stress estimate of $\Delta S \sim 20$ bar $(2 \mathrm{MPa})$ and consequently $S_{3}=235$ bar $\left(23.5 \frac{\mathrm{MPa}}{\mathrm{km}}\right)$ and $S_{2}=245 \mathrm{bar}\left(24.5 \frac{\mathrm{MPa}}{\mathrm{km}}\right)$ at $1 \mathrm{~km}$ depth for the same gradient of $S_{1}$ as used above. 


\section{References}

Abe, Y., Ohkura, T., Shibutani, T., Hirahara, K., and Kato, M. (2010). Crustal structure beneath Aso Caldera, Southwest Japan, as derived from receiver function analysis. Journal of Volcanology and Geothermal Research, 195(1):1 - 12.

Anderson, D., Minster, B., and Cole, D. (1974). The effect of oriented cracks on seismic velocities. Journal of Geophysical Research, 79(401):1-4015.

Ando, M., Ishikawa, Y., and Wada, H. (1980). S-wave anisotropy in the upper mantle under a volcanic area in Japan. Nature, 286:43-46.

Asaue, H., Koike, K., Yoshinaga, T., and Takakura, S. (2006). Magnetotelluric resistivity modeling for 3D characterization of geothermal reservoirs in the Western side of Mt. Aso, SW Japan. Journal of Applied Geophysics, 58(4):296 - 312. Electrical and Electromagnetic Studies in Geothermally Active Regions.

Aso Volcanological Laboratory (2007). Recent Activity of Aso Volcano (Jan - Dec 2007). Rep. Coordinating Committee for Prediction of Volcanic Eruption. In Japanese.

Audoine, E., Savage, M., and Gledhill, K. (2000). Seismic anisotropy from local earthquakes in the transition region from a subduction to a strike-slip plate boundary, New Zealand. Journal of Geophysical Research, 105(B4):8013-8033.

Audoine, E., Savage, M., and Gledhill, K. (2004). Anisotropic structure under a barc arc spreading region, the Taupo Volcanic Zone, New Zealand. Journal of Geophysical Research, 109(B11305).

Babuska, V. and Cara, M. (1991). Seismic anisotropy in the Earth, volume 10 of Modern Approaches in Geophysics. Kluwer Academic Publishers.

Beavan, J. (2005). Noise properties of continuous GPS data from concrete pillar geodetic monuments in New Zealand and comparison with data from U.S. deep drilled braced monuments. Journal of Geophysical Research, 110(B08410).

Beavan, J. and Haines, J. (2001). Contemporary horizontal velocity and strain rate fields of the Pacific-Australien plate boundary zone through New Zealand. Journal of Geophysical Research, 106(B1):741-770.

Beavan, J., Wallace, L., Fletcher, H., and Douglas, A. (2008). Slow Slip Events on the Hikurangi Subduction Interface, New Zealand. In Tregoning, P. and C.Rizos, editors, Dynamic Planet - Monitoring and Understanding a Dynamic Planet with Geodetic and Oceanographic Tools IAG Symposium Cairns, Australia 22-26 August, 2005, volume 130, pages 438-444. Springer Berlin Heidelberg. 
Bianco, F. and Zaccarelli, L. (2009). A reappraisal of shear wave splitting parameters from Italian active volcanic areas through a semiautomatic algorithm. Journal of Seismology, 13(2):253-266.

Bibby, H., Caldwell, T., Davey, F., and Webb, T. (1995). Geophysical evidence on the structure of the Taupo Volcanic Zone and its hydrothermal circulation. Journal of Volcanology and Geothermal Research, 68(1-3):29 - 58. Taupo Volcanic Zone, New Zealand.

Boness, N. L. and Zoback, M. D. (2006). A multiscale study of the mechanisms controlling shear velocity anisotropy in the San Andreas Fault Observatory at Depth. Geophysics, 71(5):F131-F146.

Booth, D. and Crampin, S. (1985). Shear-wave polarizations on a curved wavefront at an isotropic free surface. Geophysical Journal of the Royal Astronomical Society, 83(1):31-45.

Brace, W. and Kohlstedt, D. (1980). Limits on Lithospheric Stress Imposed by Laboratory Experiments. Journal of Geophysical Research, 85(B11):6248-6252.

Bryan, C., Sherburn, S., Bibby, H., Bannister, S., and Hurst, A. (1999). Shallow seismicity of the central Taupo Volcanic Zone, New Zealand: its distribution and nature. New Zealand Journal of Geology and Geophysics, 42:533-542.

Budiansky, B. and O'Connell, R. (1976). Elastic moduli of a cracked solid. International Journal of Solids and Structures, 12(2):81-97.

Cardozo, N. and Allmendinger, R. (2009). SSPX: A program to compute strain from displacement/velocity data. Computers and Geosciences, 35:1343-1357.

Carn, S., Krueger, A., Krotkov, N., Yang, K., and Evans, K. (2009). Tracking volcanic sulfur dioxide clouds for aviation hazard mitigation. Natural Hazards, 51:325-343.

Cayol, V. and Cornet, F. (1998). Effects of topography on the interpretation of the deformation field of prominent volcanoes-Application to Etna. Geophysical Research Letters, 25(11):1979-1982.

Chatterjee, S., Pitt, A., and Iyer, H. (1985). Vp/Vs ratios in the Yellowstone national park region, Wyoming. Journal of Volcanology and Geothermal Research, 26(34):213-230.

Chester, D., Degg, M., Duncan, A., and Guest, J. (2001). The increasing exposure of cities to the effects of volcanic eruptions: a global survey. Environmental Hazards, 2:89-103. 
Chiarabba, C. and Moretti, M. (2006). An insight into the unrest phenomena at the Campi Flegrei caldera from Vp and Vp/Vs tomography. Terra Nova, 18(6):373379 .

Cole, J., Darby, D., and Stern, T. (1995). Taupo Volcanic Zone and Central Volcanic Region - Backarc Structures of North Island, New Zealand. In Taylor, B., editor, Backarc Basins: Tectonics and Magmatism. Plenum Press.

Correa-Mora, F., DeMets, C., Cabral-Cano, E., Diaz-Molina, O., and MarquezAzua, B. (2009). Transient deformation in southern Mexico in 2006 and 2007: Evidence for distinct deep-slip patches beneath Guerrero and Oaxaca. Geochemistry Geophysics Geosystems, 10(2):Q02S12.

Crampin, S. (1978). Seismic-wave propagation through a cracked solid: polarization as a possible dilatancy diagnostic. Geophysical Journal of the Royal Astronomical Society, 53(3):467-496.

Crampin, S. (1981). A review of wave motion in anisotropic and cracked elasticmedia. Wave Motion, 3(4):343 - 391.

Crampin, S. (1985). Evaluation of anisotropy by shear-wave splitting. Geophysics, $50(1): 142-152$.

Crampin, S. (1994). The fracture criticality of crustal rocks. Geophysical Journal International, 118:428-438.

Crampin, S. and Lovell, J. (1991). A decade of shear-wave splitting in the Earth's crust: What does it mean? What use can we make of it? And what should we do next? Geophysical Journal International, 107:387-407.

Crampin, S. and Peacock, S. (2005). A review of shear-wave splitting in the compliant crack-critical anisotropic Earth. Wave Motion, 41(1):59 - 77.

Crotwell, H., Owens, T., and Ritsema, J. (1999). The TauP Toolkit: Flexible Seismic Travel-Time and Raypath Utilities. Seismological Research Letters, 70(2):154-160.

Darby, D., Hodgkinson, K., and Blick, G. (2000). Geodetic measurement of deformation in the Taupo Volcanic Zone, New Zealand: the north Taupo network revisited. New Zealand Journal of Geology and Geophysics, 43:157-170.

DeMets, C., Gordon, R., Argus, D., and Stein, S. (1994). Effect of recent revisions to the geomagnetic reversal time scale on estimates of current plate motions. Geophysical Research Letters, 21(20):2191-2194.

Dieterich, J., Cayol, V., and Okubo, P. (2000). The use of earthquake rate changes as a stress meter at Kilauea volcano. Nature, 408(6811):457-460. 
do Nascimento, A., Pearce, R., and Takeya, M. (2002). Local shear wave observations in João Câmara, northeast Brazil. Journal of Geophysical Research, 107(B10):2232.

Dong, D., Fang, P., Bock, Y., Cheng, M., and Miyazaki, S. (2002). Anatomy of apparent seasonal variations from GPS-derived site position time series. Journal of Geophysical Research, 107(B4).

Douglas, A., Beavan, J., Wallace, L., and Townend, J. (2005). Slow slip on the northern Hikurangi subduction interface, New Zealand. Geophysical Research Letters, 32(L16305).

Du, W., Thurber, C., and Eberhart-Phillips, D. (2004). Earthquake relocation using cross-correlation time delay estimates verified with the bispectrum method. Bulletin of the Seismological Society of America, 94(3):856.

Dvorkin, J., Mavko, G., and Nur, A. (1999). Overpressure detection from compressional-and shear-wave data. Geophysical Research Letters, 26(22):34173420 .

El-Rabbany, A. (2002). Introduction to GPS: the global positioning system. Artech House Publishers.

Elkibbi, M. and Rial, J. (2005). The Geysers geothermal field: results from shearwave splitting analysis in a fractured reservoir. Geophysical Journal International, 162:1024-1035.

Flesch, L., Holt, W., Silver, P., Stephenson, M., Wang, C., and Chan, W. (2005). Constraining the extent of crust-mantle coupling in central Asia using GPS, geologic, and shear wave splitting data. Earth and Planetary Science Letters, 238(12):248-268.

Fouch, M. and Rondenay, S. (2006). Seismic anisotropy beneath stable continental interiors. Physics of the Earth and Planetary Interiors, 158:292-320.

Geographical Survey Institute (2004). Crustal deformations around Aso Volcano. Report of Coordinating Committee for Prediction of Volcanic Eruption, (88):106110. In Japanese.

Gerst, A. (2003). Temporal changes in seismic anisotropy as a new eruption forecasting tool? Master's thesis, Victoria University of Wellington.

Goldstein, P. and Snoke, A. (2005). SAC Availability for the IRIS Community. Incorporated Institutions for Seismology Data Management Center Eletronic Newsletter, $7(1): 1$. 
Goree, F. (1710). A Relation of a New Island, Which Was Raised up from the Bottom of the Sea, on the 23d of May 1707. in the Bay of Santorini, in the Archipelago. Written by Father Goree (a Jesuit) an Eye-Witness. Philosophical Transactions, 27(325-336):354.

Greve, S. M., Savage, M. K., and Hofmann, S. D. (2008). Strong variations in seismic anisotropy across the Hikurangi subduction zone, North Island, New Zealand. Tectonophysics, 462(1-4):7 - 21. Seismic Anisotropy and Geodynamics of the Lithosphere-Asthenosphere System.

Gutscher, M.-A. and Lallemand, S. (1999). Birth of a major strike-slip fault in SW Japan. Terra Nova, 11:203-209.

Hartop, M. (1693). A Letter from Mr. Martin Hartop at Naples, to the Publisher. Together with an Account of the Late Earthquake in Sicily. Philosophical Transactions (1683-1775), 17:827-829.

Hase, H., Hashimoto, T., Sakanaka, S., Kanda, W., and Tanaka, Y. (2005). Hydrothermal system beneath Aso volcano as inferred from self-potential mapping and resistivity structure. Journal of Volcanology and Geothermal Research, 143(4):259 - 277 .

Hess, H. (1964). Seismic anisotropy of the uppermost mantle under oceans. Nature, 203(4965):629-631.

Hiramatsu, Y., Iwatsuki, K., Ueyama, S., Iidaka, T., and the Japanese University Group of the Joint Seismic Observations at NKTZ (2010). Spatial variation in shear wave splitting of the upper crust in the zone of inland high strain rate, central Japan. Earth, Planets, and Space, 62:675-684.

Hirose, F., Nakajima, J., and Hasegawa, A. (2008). Three-dimensional seismic velocity structure and configuration of the Philippine Sea slab in southwestern Japan estimated by double-difference tomography. Journal of Geophysical Research, 113(B9):B09315.

Hofmann-Wellenhof, B., Lichtenegger, H., and Collins, J. (1993). Global positioning System. Theory and Practice. Springer-Verlag.

Holtkamp, S. and Brudzinski, M. (2010). Determination of slow slip episodes and strain accumulation along the Cascadia margin. Journal of Geophysical Research, 115(B00A17).

Hurst, A., Bibby, H., and Robinson, R. (2002). Earthquake focal mechanisms in the central Taupo Volcanic Zone and their relation to faulting and deformation. New Zealand Journal of Geology and Geophysics, 45:527-536. 
Hurst, T., Scott, B., Werner, C., Stevens, N., and Cowan, H. (2004). Monitoring New Zealand volcanoes. Tephra, 21.

Ito, H., DeVilbiss, J., and Nur, A. (1979). Compressional and shear waves in saturated rock during water-steam transition. Journal of Geophysical Research, 84(B9):4731-4735.

Jacobs, K., Smith, E., and Savage, M. (2009). Temporal evolution of earthquake sequences (swarms) in the Central Volcanic Region, New Zealand. American Geophysical Union, Fall Meeting 2009, abstract \#NG23B-1090.

Jaeger, J., Cook, N., Zimmerman, R., and Zimmerman, R. (2007). Fundamentals of rock mechanics. Wiley-Blackwell.

Johnson, J., Prejean, S., Savage, M., and Townend, J. (2010). Anisotropy, repeating earthquakes, and seismicity associated with the 2008 eruption of Okmok volcano, Alaska. Journal of Geophysical Research, 115(null).

Johnson, J., Savage, M., and Townend, J. (2011). A Benchmark of Seismic Anisotropy at Mount Ruapehu Volcano, New Zealand. manuscript in preparation.

Jolly, A., Sherburn, S., Jousset, P., and Kilgour, G. (2010). Eruption source processes derived from seismic and acoustic observations of the 25 September 2007 Ruapehu eruption - North Island, New Zealand. Journal of Volcanology and Geothermal Research, 191:33-45.

Jolly, G., Beavan, J., Christenson, B., Ellis, S., Jolly, A., Miller, C., Peltier, A., Scott, B., Sherburn, S., Wallace, L., and McCaffrey, R. (2008). What constitutes unrest at Taupo caldera, New Zealand. EOS Transactions AGU, 89(53):Fall Meet. Suppl., Abstract V44A-07.

Jung, H., Mo, W., and Green, H. (2008). Upper mantle seismic anisotropy resulting from pressure-induced slip transition in olivine. Nature Geoscience, 2.

Kamata, H. (1989). Volcanic and structural history of the Hohi volcanic zone, central Kyushu, Japan. Bulletin of Volcanology, 51(5):315-332.

Kamata, H. and Kodama, K. (1994). Tectonics of an arc-arc junction: an example from Kyushu Island at the junction of the Southwest Japan Arc and the Ryukyu Arc. Tectonophysics, 233(1-2):69-81.

Kanda, W., Tanaka, Y., Utsugi, M., Takakura, S., Hashimoto, T., and Inoue, H. (2008). A preparation zone for volcanic explosions beneath Naka-dake crater, Aso volcano, as inferred from magnetotelluric surveys. Journal of Volcanology and Geothermal Research, 178(1):32 - 45. Dynamics of Volcanic Explosions: Field 
Observations, Experimental Constraints and Integrated Modelling of Volcanic Explosions: Field Observations, Experimental Constraints and Integrated Modelling.

Kaneko, K., Kamata, H., Koyaguchi, T., Yoshikawa, M., and Furukawa, K. (2007). Repeated large-scale eruptions from a single compositionally stratified magma chamber: An example from Aso volcano, Southwest Japan. Journal of Volcanology and Geothermal Research, 167(1-4):160 - 180. Large Silicic Magma Systems.

Kaneshima, S. and Ando, M. (1989). An Analysis of Split Shear Waves Observed Above Crustal and Uppermost Mantle Earthquakes Beneath Shikoku, Japan: Implications in Effective Depth Extent of Seismic Anisotropy. Journal of Geophysical Research, 94(B10):14,077-14,092.

Kaneshima, S., Ito, H., and M.Sugihara (1989). Shear wave polarization anisotropy observed in a rift zone in Japan. Tectonophysics, 157:281-300.

Kato, A., Iidaka, T., Ikuta, R., Yoshida, Y., Katsumata, K., Iwasaki, T., Sakai, S., Thurber, C., Tsumura, N., Yamaoka, K., et al. (2010). Variations of fluid pressure within the subducting oceanic crust and slow earthquakes. Geophysical Research Letters, 37(14):L14310.

Kendall, J.-M. (1994). Teleseismic arrivals at a mid-ocean ridge: Effects of mantle melt and anisotropy. Geophysical Research Letters, 21(4):301-304.

Kennett, B., Engdahl, E., and Buland, R. (1995). Constraints on seismic velocities in the Earth from traveltimes. Geophysical Journal International, 122:108-124.

Kita, I., Kai, T., Itoi, R., Ishida, M., and Ueda, A. (2009). Magmatic fluid input to the Kuju-Iwoyama hydrothermal system prior to the 1995 eruption of the Kuju volcano (Kyushu, Japan). Geothermics, 38(3):294 - 302.

Kubotera, A. and Mitsunami, T. (1980). An earthquake swarm in the northern part of the Aso caldera and migration of their foci. Tectonophysics, 70(3-4):223-236.

Kurashimo, E. and Hirata, N. (2004). Low Vp and Vp/Vs zone beneath the northern Fossa Magna basin, central Japan, derived from a dense array observation. Earth, Planets, and Space, 56(12):1301-1308.

Leary, P., Crampin, S., and McEvilly, T. (1990). Seismic Fracture Anisotropy in the Earth's Crust: An Overview. Journal of Geophysical Research, 95(B7):11,10511,114 .

Lev, E., Long, M. D., and van der Hilst, R. D. (2006). Seismic anisotropy in Eastern Tibet from shear wave splitting reveals changes in lithospheric deformation. Earth and Planetary Science Letters, 251(3-4):293 - 304. 
Levin, V., Okaya, D., and Park, J. (2007). Shear wave birefringence in wedge-shaped anisotropic regions. Geophysical Journal International, 168:275-286.

Li, G., Kimura, F., Sato, T., and Huang, D. (2008). A composite analysis of diurnal cycle of GPS precipitable water vapor in central Japan during Calm Summer Days. Theoretical and Applied Climatology, 92(1):15-29.

Lin, G. and Shearer, P. (2009). Evidence for water-filled cracks in earthquake source regions. Geophysical Research Letters, 36(17):L17315.

Lin, J. and Stein, R. (2004). Stress triggering in thrust and subduction earthquakes and stress interaction between the southern San Andreas and nearby thrust and strike-slip faults. Journal of Geophysical Research, 109(B2):B02303.

Liu, E. and Crampin, S. (1990). Effects of the internal shear wave window: comparison with anisotropy induced splitting. Journal of Geophysical Research, 95:11-275.

Liu, L. and Zoback, M. (1992). The effect of topography on the state of stress in the crust: Application to the site of the Cajon Pass Scientific Drilling Project. Journal of Geophysical Research, 97(B4):5095-5108.

Liu, Z., Owen, S., Dong, D., Lundgren, P., Webb, F., Hetland, E., and Simons, M. (2010). Integration of transient strain events with models of plate coupling and areas of great earthquakes in southwest Japan. Geophysical Journal International, 181(3):1292-1312.

Long, M. and Silver, P. (2009). Shear wave splitting and mantle anisotropy: Measurements, interpretations, and new directions. Surveys in Geophysics, 30:407461. $10.1007 / \mathrm{s} 10712-009-9075-1$.

Loveless, J. and Meade, B. (2010). Geodetic imaging of plate motions, slip rates, and partitioning of deformation in Japan. Journal of Geophysical Research, 115(B02410).

Lucente, F., De Gori, P., Margheriti, L., Piccinini, D., Di Bona, M., Chiarabba, C., and Piana Agostinetti, N. (2010). Temporal variation of seismic velocity and anisotropy before the 2009 MW 6.3 L'Aquila earthquake, Italy. Geology, $38(11): 1015$.

Luckett, R., Baptie, B., Ottemoller, L., and Thompson, G. (2007). Seismic Monitoring of the Soufrière Hills Volcano, Montserrat. Seismological Research Letters, 78(2):192-200.

Lund, B. and Townend, J. (2007). Calculating horizontal stress orientations with full or partial knowledge of the tectonic stress tensor. Geophysical Journal International, 170:1328-1335. 
Mainprice, D., Tommasi, A., Couvy, H., Cordier, P., and Frost, D. (2005). Pressure sensitivity of olivine slip systems and seismic anisotropy of Earth's upper mantle. Letters to Nature, 433:731-733.

Martín-Velázquez, S., de Vicente, G., and Elorza, F. (2009). Intraplate stress state from finite element modelling: The southern border of the Spanish Central System. Tectonophysics, 473(3-4):417-427.

Martínez-Arévalo, C., Bianco, F., Ibáñez, J., and Pezzo, E. D. (2003). Shallow seismic attenuation and shear-wave splitting in the short period range of Deception Island volcano (Antarctica). Journal of Volcanology and Geothermal Research, 128:89-113.

Masterlark, T., Haney, M., Dickinson, H., Fournier, T., and Searcy, C. (2010). Rheological and structural controls on the deformation of Okmok volcano, Alaska: FEMs, InSAR, and ambient noise tomography. Journal of Geophysical Research, 115(B02409).

Mazengarb, C. and Speden, I. (2000). Geology of the Raukumara area. 1: 250000 geological map 6. Lower Hutt, New Zealand, Institute of Geological \& Nuclear Sciences Limited.

McGinty, P., Reyners, M., and Robinson, R. (2000). Stress directions in the shallow part of the Hikurangi subduction zone, New Zealand, from the inversion of earthquake first motions. Geophysical Journal International, 142(2):339-350.

McTigue, D. (1987). Elastic stress and deformation near a finite spherical magma body: resolution of the point source paradox. Journal of Geophysical Research, 92(B12):12931.

Means, W. (1976). Stress and strain: basic concepts of continuum mechanics for geologists. Springer-Verlag, New York.

Miller, D. and Dunne, T. (1996). Topographic perturbations of regional stresses and consequent bedrock fracturing. Journal of Geophysical Research, 101(B11):25523.

Misra, P. and Enge, P. (2006). Global Positioning System: Signals, Measurements and Performance Second Edition. Lincoln, MA: Ganga-Jamuna Press.

Miyabuchi, Y., Ikebe, S., and Watanabe, K. (2008). Geological constraints on the 2003-2005 ash emissions from the Nakadake crater lake, Aso Volcano, Japan. Journal of Volcanology and Geothermal Research, 178(2):169 - 183. Volcanic lakes and environmental impacts of volcanic fluids.

Miyazaki, S., Hatanaka, Y., Sagiya, T., and Tada, T. (1998). The nationwide GPS array as an earth observation system. Bulletin of the Geographical Survey Institute, $44: 11-22$. 
Miyazaki, S. and Heki, K. (2001). Crustal velocity field of southwest Japan - Subduction and arc-arc collision. Journal of Geophysical Research, 106:4305-4326.

Miyazaki, S., Iwabuchi, T., Heki, K., and Naito, I. (2003). An impact of estimating tropospheric delay gradients on precise positioning in the summer using the Japanese nationwide GPS array. J. geophys. Res, 108(2335):1994-2006.

Miyazaki, S., Saito, T., Sasaki, M., Hatanaka, Y., and Iimura, Y. (1997). Expansion of GSI's nationwide GPS array. Bulletin of the Geographical Survey Institute, 43:23-34.

Miyazaki, S., Tsuji, H., Hatanaka, Y., Abe, Y., Yoshimura, A., Kamada, K., Kobayashi, K., Morishita, H., and Iimura, Y. (1996). Establishment of the nationwide GPS array (GRAPES) and its initial results on the crustal deformation of Japan. Bulletin of the Geographical Survey Institute, 42:27-41.

Mogi, K. (1958). Relations between the eruptions of various volcanoes and the deformations of the ground surfaces around them. Bulletin of the Earthquake Research Institute, 36:99-134.

Mordret, A., Jolly, A., Duputel, Z., and Fournier, N. (2010). Monitoring of phreatic eruptions using Interferometry on Retrieved Cross-Correlation Function from Ambient Seismic Noise: Results from Mt. Ruapehu, New Zealand. Journal of Volcanology and Geothermal Research, 191(1-2):46-59.

Mori, T., Sudo, Y., Tsutsui, T., and Yoshikawa, S. (2008). Characteristics of isolated hybrid tremor (HBT) during a calm activity period at Aso Volcano. Bulletin of Volcanology, 70:1031-1042.

Murray, J., Hudnall, L., Matus, A., Krueger, A., and Trepte, C. (n.d.). The detection, characterization and tracking of recent Aleutian Island volcanic ash plumes and the assessment of their impact on aviation. Retrieved Aug 07, 2010 from http://ntrs.nasa.gov/archive/nasa/casi.ntrs.nasa.gov/20100003407_2010003449.pdf.

Nakajima, J., Matsuzawa, T., Hasegawa, A., and Zhao, D. (2001a). Seismic imaging of arc magma and fluids under the central part of northeastern Japan. Tectonophysics, 341(1-4):1-17.

Nakajima, J., Matsuzawa, T., Hasegawa, A., and Zhao, D. (2001b). Threedimensional structure of $V_{p}, V_{s}$, and $V_{p} / V_{s}$ beneath northeastern Japan - Implications for arc magmatism and fluids. Journal of Geophysical Research, 106(21):84357.

Nicol, A. and Wallace, L. (2007). Temporal stability of deformation rates: Comparison of geological and geodetic observations, Hikurangi subduction margin, New Zealand. Earth and Planetary Science Letters, 258(3-4):397-413. 
Nur, A. (1972). Dilatancy, pore fluids, and premonitory variations of $t_{s} / t_{p}$ travel times. Bulletin of the Seismological Society of America, 62(5):1217.

Nur, A. and Simmons, G. (1969a). Stress-induced velocity anisotropy in rock: An experimental study. Journal of Geophysical Research, 74(27).

Nur, A. and Simmons, G. (1969b). The effect of saturation on velocity in low porosity rocks. Earth and Planetary Science Letters, 7(2):183-193.

Nuttli, O. (1961). The effect of the earth's surface on the S-wave particle motion. Bulletin of the Seismological Society of America, 51(2):237-246.

Ohkura, T. and Oikawa, J. (2008). GPS observation of crustal movements at Aso Volcano. In Programme and Abstract: The Volcanological Society of Japan 2008 fall meeting, page 58. In Japanese.

Okubo, Y. and Shibuya, A. (1993). Thermal and crustal structure of the Aso volcano and surrounding regions constrained by gravity and magnetic data, Japan. Journal of Volcanology and Geothermal Research, 55(3-4):337-350.

Ono, K., Watanabe, K., Hoshizumi, H., and Ikebe, S. (1995). Ash eruption of the Naka-dake crater, Aso volcano, southwestern Japan. Journal of Volcanology and Geothermal Research, 66(1-4):137-148.

Ozacar, A. A. and Zandt, G. (2009). Crustal structure and seismic anisotropy near the San Andreas Fault at Parkfield, California. Geophysical Journal International, 178(1098-1104).

Ozawa, S., Murakami, M., and Tada, T. (2001). Time-dependent inversion study of the slow thrust event in the Nankai trough subduction zone, southwestern Japan. Journal of Geophysical Research, 106(B1):787-802.

Park, J. and Levin, V. (2002). Seismic Anisotropy: Tracing Plate Dynamics in the Mantle. Science, 296(485).

Pastori, M., Piccinini, D., Margheriti, L., Improta, L., Valoroso, L., Chiaraluce, L., and Chiarabba, C. (2009). Stress aligned cracks in the upper crust of the Val d'Agri region as revealed by shear wave splitting. Geophysical Journal International, 179:601-614.

Reyners, M. (2010). Stress and strain from earthquakes at the southern termination of the Taupo Volcanic Zone, New Zealand. Journal of Volcanology and Geothermal Research, 190(1-2):82 - 88. Making and Breaking the Arc: a volume in honour of Professor John Gamble.

Reyners, M., Eberhart-Phillips, D., and Stuart, G. (1999). A three-dimensional image of shallow subduction: crustal structure of the Raukumara Peninsula, New Zealand. Geophysical Journal International, 137(3):873-890. 
Reyners, M., Eberhart-Phillips, D., Stuart, G., and Nishimura, Y. (2006). Imaging subduction from the trench to $300 \mathrm{~km}$ depth beneath the central North Island, New Zealand, with $V p$ and $V p / V s$. Geophysical Journal International, 165:565583.

Reyners, M. and McGinty, P. (1999). Shallow subduction tectonics in the Raukumara Peninsula, New Zealand, as illuminated by earthquake focal mechanisms. Journal of Geophysical Research, 104(B2):3025-3034.

Rowland, J. and Sibson, R. (2001). Extensional fault kinematics within the Taupo Volcanic Zone, New Zealand: soft-linked segmentation of a continental rift system. New Zealand Journal of Geology and Geophysics, 44:271-283.

Sagiya, T., Miyazaki, S., and Tada, T. (2000). Continuous GPS array and presentday crustal deformation of Japan. Pure and Applied Geophysics, 157:2303-2322.

Savage, J. (1983). A dislocation model of strain accumulation and release at a subduction zone. Journal of Geophysical Research, 88(B6):4984-4996.

Savage, M. (1999). Seismic anisotropy and mantle deformation: What have we learned from shear wave splitting? Reviews of Geophysics, 37(1):65-106.

Savage, M., Peppin, W., and Vetter, U. (1990). Shear Wave Anisotropy and Stress Direction in and Near Long Valley Caldera, California, 1979-1988. Journal of Geophysical Research, 95(B7):11,165-11,177.

Savage, M., Sheehan, A., and Lerner-Lam, A. (1996). Shear wave splitting across the Rocky Mountain Front. Geophysical Research Letters, 23(17):2267-2270.

Savage, M., Shih, X., Meyer, R., and Aster, R. (1989). Shear-wave anisotropy of active tectonic regions via automated S-wave polarization analysis. Tectonophysics, 165:279-292.

Savage, M., Wessel, A., Teanby, N., and Hurst, T. (2010a). Automatic measurement of shear wave splitting and applications to time varying anisotropy at Mt. Ruapehu volcano, New Zealand. Journal of Geophysical Research, 115(B12):B12321.

Savage, M. K., Ohkura, T., Umakoshi, K., Shimizu, H., Kohno, Y., Iguchi, M., Wessel, A., and Mori, J. (2008). Variations in Seismic Anisotropy with time on Volcanoes in Kyushu Island, Southern Japan. AGU Fall Meeting Abstracts, pages A1814+.

Savage, M. K., Ohminato, T., Aoki, Y., Tsuji, H., and Greve, S. M. (2010b). Stress magnitude and its temporal variation at Mt. Asama Volcano, Japan, from seismic anisotropy and GPS. Earth and Planetary Science Letters, 290(3-4):403 - 414. 
Schwartz, S. and Rokosky, J. (2007). Slow slip events and seismic tremor at circumpacific subduction zones. Reviews of Geophysics, 45(RG3004).

Segall, P. (2010). Earthquake and volcano deformation. Princeton Univ Pr.

Shaw, G. and Healy, J. (1962). Geology of the Bay of Plenty - Taupo region. Town and Country Planning Banch, Ministry of Works, Wellington.

Sherburn, S., Townend, J., Arnold, R., and Woods, L. (2009). EQC Project 08/550 - Establishing a Spatiotemporal Benchmark for Ongoing Crustal Stress Monitoring in the Southern Taupo Volcanic Zone. Consultancy Report 2009/185, GNS Science.

Sibson, R. and Rowland, J. (2003). Stress, fluid pressure and structural permeability in seismogenic crust, North Island, New Zealand. Geophysical Journal International, 154:584-594.

Silver, P. and Chan, W. (1991). Shear wave splitting and subcontinental mantle deformation. Journal of Geophysical Research, 96(B10):16,429-16,454.

Silver, P. and Savage, M. (1994). The interpretation of shear-wave splitting parameters in the presence of two anisotropic layers. Geophysical Journal International, 119(3):949-963.

Smith, E., Williams, T., and Darby, D. (2007). Principal component analysis and modeling of the subsidence of the shoreline of Lake Taupo, New Zealand, 19831999: Evidence for dewatering of a magmatic intrusion? Journal of Geophysical Research, 112(B08406).

Spinks, K., Acocella, V., Cole, J., and Bassett, K. (2005). Structural control of volcanism and caldera development in the transtensional Taupo Volcanic Zone, New Zealand. Journal of Volcanology and Geothermal Research, 144:7-22.

Stein, S. and Wysession, M. (2003). An introduction to seismology, earthquakes, and earth structure. Wiley-Blackwell.

Stern, T. (1985). A back-arc basin formed within continental lithosphere: The Central Volcanic region of New Zealand. Tectonophysics, 112(1-4):385 - 409. Structures and Processes in Subduction Zones.

Stern, T. (1987). Asymmetric back-arc spreading, heat flux and structure associated with the Central Volcanic Region of New Zealand. Earth and Planetary Science Letters, 85(1-3):265 - 276.

Strang, G. and Borre, K. (1997). Linear algebra, geodesy, and GPS. Wellesley Cambridge Pr. 
Styles, K. (2009). Shear wave attenuation structure and anisotropy in the Hikurangi subduction zone, central North Island, New Zealand. PhD thesis, School of Earth and Environment, The University of Leeds.

Sudo, Y. (1991). An attenuating structure beneath the Aso Caldera determined from the propagation of seismic waves. Bulletin of Volcanology, 53:99-111.

Sudo, Y. and Hurst, A. W. (1998). Temperature changes at depths to 150 metres near the active crater of Aso Volcano: preliminary analysis of seasonal and volcanic effects. Journal of Volcanology and Geothermal Research, 81(1-2):159 172 .

Sudo, Y. and Kong, L. (2001). Three-dimensional seismic velocity structure beneath Aso Volcano, Kyushu, Japan. Bulletin of Volcanology, 63:326-344.

Sudo, Y. and Matsumoto, Y. (1998). Three-dimensional P-wave velocity structure in the upper crust beneath Kuju Volcano, central Kyushu, Japan. Bulletin of Volcanology, 60(3):147-159.

Sudo, Y., Ono, H., Hurst, A. W., Tsutsui, T., Mori, T., Nakaboh, M., Matsumoto, Y., Sako, M., Yoshikawa, S., Tanaka, M., Kobayashi, Y., Hashimoto, T., Hoka, T., Yamada, T., Masuda, H., and Kikuchi, S. (1998). Seismic activity and ground deformation associated with 1995 phreatic eruption of Kuju Volcano, Kyushu, Japan. Journal of Volcanology and Geothermal Research, 81(3-4):245 - 267.

Sudo, Y., Tsutsui, T., Nakaboh, M., Yoshikawa, M., Yoshikawa, S., and Inoue, H. (2006). Ground Deformation and Magma Reservoir at Aso Volcano: Location of Deflation Source Derived from Long-term Geodetic Surveys. Kazan, 51(5):291309. In Japanese.

Swanson, D. (2008). Hawaiian oral tradition describes 400 years of volcanic activity at Kilauea. Journal of Volcanology and Geothermal Research, 176(3):427-431.

Takai, F., Matsumoto, T., Toriyama, R., and Girdler, R. (1965). Geology of Japan. Geophysical Journal of the Royal Astronomical Society, 9(2-3):283-284.

Takayama, H. and Yoshida, A. (2007). Crustal deformation in Kyushu derived from GEONET data. Journal of Geophysical Research, 112(B6):B06413.

Takei, Y. (2002). Effect of pore geometry on $V_{P} / V_{S}$ : From equilibrium geometry to crack. Journal of Geophysical Research, 107(B2):2043.

Tanguy, J.-C., Ribière, C., Scarth, A., and Tjetjep, W. (1998). Victims from volcanic eruptions: a revised database. Bulletin of Volcanology, 60:137-144.

Tatham, R. (1982). $V_{p} / V_{s}$, and lithology. Geophysics, 47(3):336-344. 
Teanby, N., Kendall, J.-M., Jones, R., and Barkved, O. (2004a). Stress-induced temporal variations in seismic anisotropy observed in microseismic data. Geophysical Journal International, 156(459-466).

Teanby, N., Kendall, J.-M., and van der Baan, M. (2004b). Automation of shearwave splitting measurements using cluster analysis. Bulletin of the Seismological Society of America, 94(2):453-463.

Terada, A., Hashimoto, T., Kagiyama, T., and Sasaki, H. (2008). Precise remotemonitoring technique of water volume and temperature of a crater lake in Aso volcano, Japan: implications for a sensitive window of a volcanic hydrothermal system. Earth Planets Space, 60:705-710.

Toda, S., Stein, R., Richards-Dinger, K., and Bozkurt, S. (2005). Forecasting the evolution of seismicity in southern California: Animations built on earthquake stress transfer. Journal of Geophysical Research, 110(B5):B05S16.

Townend, J. and Zoback, M. (2006). Stress, strain, and mountain building in central Japan. Journal of Geophysical Research, 111(B03411).

Tsutsui, T. and Sudo, Y. (2004). Seismic reflectors beneath the central cones of Aso Volcano, Kyushu, Japan. Journal of Volcanology and Geothermal Research, 131(1-2):33 - 58 .

Unglert, K., Savage, M., and Fournier, N. (2009). Crustal cracks in areas of active deformation: Correlation of GPS and seismic anisotropy. In Barrell, D. and Tulloch, A., editors, Programme and abstracts, Geosciences 09 Conference, Oamaru, New Zealand. Geological Society of New Zealand Miscellaneous Publication, volume 128A, page 218.

Upton, P., Koons, P., and Eberhart-Phillips, D. (2003). Extension and partitioning in an oblique subduction zone, New Zealand: Constraints from three-dimensional numerical modeling. Tectonics, 22(6):1068.

Vanorio, T., Virieux, J., Capuano, P., and Russo, G. (2005). Three-dimensional seismic tomography from $P$ wave and $S$ wave microearthquake travel times and rock physics characterization of the Campi Flegrei Caldera. Journal of Geophysical Research, 110(B3):B03201.

Villamor, P. and Berryman, K. (2001). A late Quaternary extension rate in the Taupo Volcanic Zone, New Zealand, derived from fault slip data. New Zealand Journal of Geology and Geophysics, 44(2):243-269.

Villamor, P. and Berryman, K. (2006). Evolution of the southern termination of the Taupo Rift, New Zealand. New Zealand Journal of Geology and Geophysics, 49:23-37. 
Villamor, P., Van Dissen, R., Alloway, B., Palmer, A., and Litchfield, N. (2007). The Rangipo fault, Taupo rift, New Zealand: An example of temporal slip-rate and single-event displacement variability in a volcanic environment. Geological Society of America Bulletin, 119(5-6):529.

Volcano (n.d.). Online Etymology Dictionary. Retrieved Feb 15, 2011 from http://www.etymonline.com/index.php?term=volcano.

Wallace, L. and Beavan, J. (2010). Diverse slow slip behavior at the Hikurangi subduction margin, New Zealand. Journal of Geophysical Research, 115(B12):B12402.

Wallace, L., Beavan, J., McCaffrey, R., and Darby, D. (2004). Subduction zone coupling and tectonic block rotations in the North Island, New Zealand. Journal of Geophysical Research, 109(B12406).

Watson, I., Oppenheimer, C., Voight, B., Francis, P., Clarke, A., Stix, J., Miller, A., Pyle, D., Burton, M., Young, S., Norton, G., Loughlin, S., Darroux, B., and Staff, M. (2000). The relationship between degassing and ground deformation at Soufrière Hills Volcano, Montserrat. Journal of Volcanology and Geothermal Research, 98:117-126.

Wessel, A. (2010). Automatic shear wave splitting measurements at Mt.Ruapehu volcano, New Zealand. Master's thesis, School of Geography, Environment and Earth Sciences, Victoria University of Wellington.

Wilson, C., Houghton, B., McWilliams, M., Lanphere, M., Weaver, S., and Briggs, R. (1995). Volcanic and structural evolution of Taupo Volcanic Zone, New Zealand: a review. Journal of Volcanology and Geothermal Research, 68:1-28.

Wolfe, C. and Silver, P. (1998). Seismic anisotropy of oceanic upper mantle: Shear wave splitting methodologies and observations. Journal of Geophysical Research, 103(B1):749-771.

Wyss, M., Gillard, D., and Liang, B. (1992). An estimate of the absolute stress tensor in Kaoiki, Hawaii. Journal of Geophysical Research, 97(B4):4763-4768.

Yamamoto, M., Kawakatsu, H., Kaneshima, S., Mori, T., Tsutsui, T., and Sudo, Y. amd Morita, Y. (1999). Detection of a crack-like conduit beneath the active crater at Aso volcano, Japan. Geophysical Research Letters, 26(24):3677-3680.

Zaccarelli, L., Pandolfi, D., Bianco, F., Saccorotti, G., Bean, C., and Pezzo, E. D. (2009). Temporal changes in seismic wave propagation towards the end of the 2002 Mt Etna eruption. Geophysical Journal International, 178:1779-1788.

Zatsepin, S. and Crampin, S. (1997). Modelling the compliance of crustal rock - I. Response of shear-wave splitting to differential stress. Geophysical Journal International, 129:477-494. 
Zinke, J. and Zoback, M. (2000). Structure-related and stress-induced shear-wave velocity anisotropy: observations from microearthquakes near the Calaveras Fault in Central California. Bulletin of the Seismological Society of America, 90(5):1305.

Zoback, M. D. and Townend, J. (2001). Implications of hydrostatic pore pressures and high crustal strength for the deformation of intraplate lithosphere. Tectonophysics, 336(1-4):19 - 30. 